\title{
Salpingectomy with delayed oophorectomy in BRCA1/2 mutation carriers
}

MARLINE HARMSEN 


\section{COLOPHON}

\section{Salpingectomy with delayed oophorectomy in BRCA1/2 mutation carriers}

The research presented in this thesis was conducted at the department of Obstetrics and Gynaecology of the Radboud university medical center, Nijmegen, the Netherlands. Parts of the research presented in this thesis were funded by the Dutch Cancer Society (KWF Kankerbestrijding).

The printing of this thesis was financially supported by the Radboud university medical center, AstraZeneca BV, Erbe Nederland BV, Mark Two Academy, ChipSoft and ANFO Medical - oncological \& gynaecological products (www.anfo.nl).

For reasons of consistency within this thesis, some terms and abbreviations have been standardized throughout the text. As a consequence, the text may differ in this respect from the papers that have been published.

Cover design: isontwerp.nl - Ilse Schrauwers

Lay-out: Gildeprint - Enschede (Nicole Nijhuis)

Printed by: Gildeprint - Enschede

ISBN: 978-94-92303-15-8

cM.G. Harmsen, 2017

All rights reserved. No part of this thesis may be reproduced, stored in a retrieval system, or transmitted,in any form or by any means, without prior permission of the author, or, when appropriate, of the publishers of the publications. 


\title{
Salpingectomy with delayed oophorectomy in BRCA1/2 mutation carriers
}

\section{Proefschrift}

\author{
ter verkrijging van de graad van doctor \\ aan de Radboud Universiteit Nijmegen \\ op gezag van de rector magnificus prof. dr. J.H.J.M. van Krieken, \\ volgens besluit van het college van decanen \\ in het openbaar te verdedigen op donderdag 22 juni 2017 \\ om 10.30 uur precies
}

door

\section{Marline Ghislaine Harmsen}

geboren op 19 februari 1987

te Nieuwegein 


\section{Promotoren}

Prof. dr. L.F.A.G. Massuger

Prof. dr. N. Hoogerbrugge-van der Linden

\section{Copromotoren}

Dr. J.A. de Hullu

Dr. R.P.M.G. Hermens

\section{Manuscriptcommissie}

Prof. dr. J.W.A. Smit

Prof. dr. L.A.L.M. Kiemeney

Prof. dr. F. Amant (Katholieke Universiteit Leuven, België) 


\section{COHTEMT}

\section{Chapter}

Page

1 Introduction and thesis outline

2 How medical choices influence quality of life of women carrying a BRCA mutation Crit Rev Oncol Hematol. 2015 Dec;96(3):555-68.

3 Very high uptake of risk-reducing salpingo-oophorectomy in BRCA1/2 mutation carriers: a single-center experience

Gynecol Oncol. 2016 Oct;143(1):113-9.

4 Peritoneal carcinomatosis after risk-reducing surgery in BRCA1/2 mutation carriers: new implications for gynecologic counseling submitted

5 Risk-reducing salpingectomy with delayed oophorectomy in BRCA1/2 mutation carriers: patients' and professionals' perspectives

Gynecol Oncol. 2015 Feb;136(2):305-10.

6 Salpingectomy with delayed oophorectomy in BRCA1/2 mutation carriers:

estimating ovarian cancer risk

Obstet Gynecol. 2016 Jun;127(6):1054-63.

7 Early salpingectomy (TUbectomy) with delayed oophorectomy to improve quality of life as alternative for risk-reducing salpingo-oophorectomy in BRCA1/2 mutation carriers (TUBA study): a prospective non-randomized multicenter study BMC Cancer. 2015 Aug 19;15(1):593.

8 A patient decision aid for risk-reducing surgery in premenopausal BRCA1/2 mutation carriers: development process and pilot testing Submitted

9 General discussion and future perspectives

10 Summary|Samenvatting

\section{Appendix}

Bibliography

Curriculum Vitae

Dankwoord 



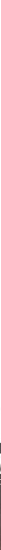

Introduction and thesis outline

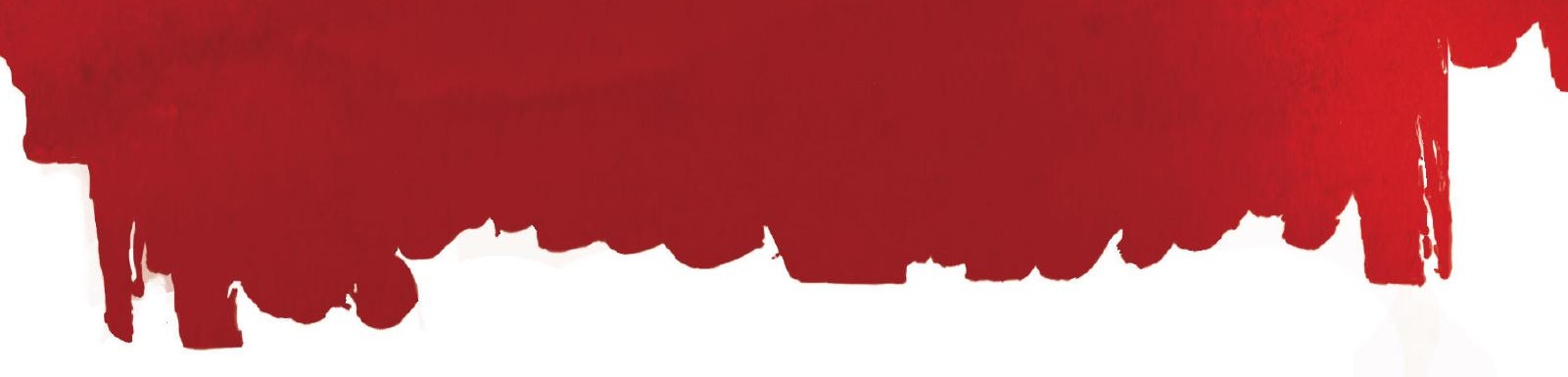




\section{IITRODICTION}

In this thesis, we focus on management options to reduce the risk of ovarian carcinoma related to $B R C A 1 / 2$ germline mutations. Current guidelines recommend to remove both ovaries and fallopian tubes during risk-reducing salpingo-oophorectomy (RRSO) around the age of 40 years. RRSO, however, is known to have several adverse effects due to acute onset of premature menopause. The magnitude of this problem in terms of how many women face premature menopause, to what extent quality of life is affected, and the residual chance to develop primary peritoneal carcinoma were evaluated in this thesis. This resulted in the need to explore the possibility and acceptability of an alternative risk-reductive strategy consisting of salpingectomy with delayed oophorectomy, of which results are also included in this thesis.

This chapter will first introduce some background knowledge on the function of BRCA genes, the epidemiology of BRCA mutations and organization of healthcare for female BRCA1/2 mutation carriers. Second, epidemiology, classification, and carcinogenesis of ovarian carcinoma are discussed. Third, we describe the options for ovarian carcinoma risk reduction in BRCA1/2 mutation carriers and their consequences with special attention to premature menopause. Finally, we outline the main objectives and content of this thesis.

\section{BRCA1/2 genes}

Pathogenic germline mutations in the two breast cancer (BRCA) susceptibility genes account for the majority of autosomal dominant inheritance of familial breast and ovarian cancer. ${ }^{1}$ The BRCA1 gene was localized to chromosome $17 q$ in the early nineties of the $20^{\text {th }}$ century and the BRCA2 gene was identified at chromosome $13 q$ a couple of years later., ${ }^{2,3}$ Both genes are tumor suppressor genes and the proteins produced by these genes play a role in an error-free DNA damage repair pathway; more specifically, in the repair of double-stranded breaks through homologous recombination. ${ }^{4}$ Thereby, functional BRCA1/2 genes help preserving the genomic integrity of the cell. ${ }^{4}$ BRCA1/2 mutation carriers are heterozygous for the mutated gene and a second hit to the unaffected allele is mostly responsible for the eventual loss of BRCA1/2 functions. By this 'loss of heterozygosity', the cell is thrown back on other DNA repair mechanisms that are often more sensitive to errors. Consequently, cells with unrepaired DNA damage are prone to transform into cancer cells.

Prevalence of $B R C A 1 / 2$ germline mutations varies from $0.2 \%$ to $0.3 \%$ in general populations to $2 \%$ among Ashkenazi Jewish people and $20 \%$ in high-risk families. ${ }^{5}$ Women carrying a BRCA1 mutation have a mean cumulative risk of 54\% (95\% confidence interval (Cl) 46-63\%) of developing breast carcinoma and 39\% (95\% Cl 34-44\%) of developing ovarian carcinoma before the age of 70 years. ${ }^{6}$ Corresponding mean cumulative risks for BRCA2 mutation carriers are 45\% (95\% Cl 38-53\%) and 16\% (95\% Cl 12-20\%) for breast and ovarian carcinoma, respectively. ${ }^{6}$ However, estimated risks vary widely by the method of analysis. For instance, point estimates of cumulative breast cancer risk to age 70 years for BRCA1 mutation carriers vary from 35\% to $83 \%$ and for BRCA2 mutation carriers 
from 41 to $86 \%$ in the same population depending on statistical method. ${ }^{7}$ Nevertheless, these risks are considerably higher than lifetime risks of women in the general population: $12.4 \%$ for breast carcinoma and $1.3 \%$ for ovarian carcinoma., Furthermore, both genes have been associated with other cancer types as well; elevated risks of pancreatic cancer and male breast and prostate cancer among BRCA2 mutation carriers are most pronounced..$^{10}$

Ovarian carcinoma related to BRCA1/2 germline mutations accounts for about 10-15\% of all ovarian carcinomas. ${ }^{11-13}$ BRCA1-related ovarian carcinoma is mostly diagnosed at earlier age (mean 51 to 53 years) than BRCA2-related (mean 55 to 60 years) or sporadic disease (mean 58 to 61 years). ${ }^{14-17}$ Current Dutch national guidelines suggest referral for genetic testing of every woman diagnosed with ovarian carcinoma, regardless of age, histology or family history. ${ }^{11,18}$

In the Netherlands, healthcare and supporting care for BRCA1/2 mutation carriers are mainly centralized in multidisciplinary Family Cancer Clinics with clinical geneticists, genetic counselors, (plastic) surgeons, medical oncologists, gynecologic oncologists, radiologists, pathologists, specialized nurses, and social workers involved. In the Radboudumc, weekly multidisciplinary team meetings are held since the year 2000 to discuss patients and their families to improve quality of care. Furthermore, nurses at our department of Obstetrics and Gynecology act as case managers and first contact person for BRCA1/2 mutation carriers to keep healthcare easily accessible. They are mainly involved in supportive care after RRSO with respect to vasomotor symptoms, sexuality, and hormone replacement therapy. Outside the hospital setting, BRCA1/2 mutation carriers seek peer support in patients' associations, patient support groups and social media forums such as Facebook.

\section{Ovarian carcinoma}

Ovarian carcinoma was diagnosed in 1266 women in the Netherlands in 2015 and was with 2.6\% the seventh most common cancer type diagnosed in women. ${ }^{19}$ However, it is the most lethal gynecologic malignancy accounting for about 1000 deaths each year. ${ }^{20}$ Ovarian carcinoma is particularly diagnosed in advanced stage, with intra- or extra-abdominal metastases in about $80 \%$ of cases. ${ }^{9}$ Primary treatment typically consists of staging or cytoreductive surgery with (neo) adjuvant platinum-based chemotherapy. However, relative 5 -year survival rates remain poor and rapidly decrease with increased stage: $92 \%$ and $29 \%$ for women with localized and metastasized disease, respectively. ${ }^{9}$ BRCA1/2 mutation carriers have been shown to have better overall and progression-free survival rates compared to non-carriers. ${ }^{21,22}$ This might be best explained by a favorable response to platinum-based chemotherapy. ${ }^{12}$ Besides, beneficial effects of poly(ADP)ribose polymerase (PARP) inhibitors for recurrent ovarian carcinoma have been reported over the last years: a novel treatment option targeting the defective DNA repair pathway in BRCA1/2 mutation carriers' tumor cells. ${ }^{23}$ This new treatment option stresses the need to find all BRCA1/2 mutation carriers among those with ovarian carcinoma. ${ }^{24,25}$ Within the group of BRCA1/2 mutation carriers, a personal history of breast cancer might worsen the prognosis of subsequent ovarian carcinoma, especially when breast cancer was treated by (adjuvant) chemotherapy. ${ }^{26}$ 
It is of utmost importance to notice that the definition "ovarian carcinoma" is not limited to carcinoma of the ovary itself, but that it is rather a collective term for carcinomas of Müllerian origin of the ovaries, fallopian tubes and the peritoneum. These carcinomas were taken together as one entity because of great similarities in morphology, prognosis and treatment. Traditionally, these carcinomas were subdivided based on histology: serous (about 70\%), endometrioid (about 10\%), clear cell (about 12\%), mucinous (about 3\%), transitional (i.e. malignant Brenner tumor, about 1\%) and undifferentiated/unclassifiable.27,28 Distribution of histological subtypes among BRCA1/2 mutation carriers does not explicitly differ from the distribution in the general population.1,15,29 Differentiation grade used to be classified according to Silverberg. ${ }^{30}$

The updated World Health Organisation classification (2014) discriminates between high-grade serous, low-grade serous, endometrioid, clear cell, mucinous, and undifferentiated carcinomas. ${ }^{31}$ Furthermore, a dichotomy between type I and type II tumors was proposed by Kurman and Shih in 2011 based on morphologic and molecular genetic studies with supposed different pathways in the carcinogenesis for either type. ${ }^{32}$ Characteristics of type I and type II tumors are described in the last paragraph of this subsection. Unfortunately, the suggested pathways are still "supposed" because the carcinogenesis of ovarian carcinoma is still not fully elucidated.

Historically, carcinomas were thought to originate from a single cell type, i.e. ovarian surface epithelium, whether or not after invagination of this epithelium into so-called cortical inclusion cysts. The incessant ovulation theory proposed by Fathalla in the 1970s describes the malignant deterioration of ovarian surface epithelium that has become vulnerable through the repeated process of damage and repair during every ovulation. ${ }^{33}$ This theory is supported by the fact that high parity and the use of oral contraceptives (i.e. lower number of lifetime ovulations) are wellestablished risk-reducing factors. However, early ovarian carcinomas or in situ lesions have been rarely identified in the ovarian surface epithelium. ${ }^{34,35}$ A second tissue type that was put forward as site of origin of ovarian carcinoma is the "secondary Müllerian system" that consists of the pelvic and lower abdominal mesothelium and the subjacent mesenchyma arising from the embryonic coelomic lining in close relation to Müllerian epithelium. It was supposed to have the ability to differentiate into Müllerian-type epithelium through a process that is called metaplasia. ${ }^{36}$

More recently, the tubal-peritoneal junction and the fimbriated end of the fallopian tubes were proposed as origin of ovarian carcinoma, mainly of the serous subtype. ${ }^{37}$ The latter was first described by Piek et al. ${ }^{38}$ after the detection of dysplastic changes in the fimbriated ends of fallopian tubes that were removed during risk-reducing salpingo-oophorectomies in BRCA1/2 mutation carriers. Many other study groups have focused on this particular site since then, and a potential cascade of p53-signatures, serous tubal intraepithelial lesions (STIL) and serous tubal intraepithelial carcinomas (STIC) has been suggested as the carcinogenic pathway of tubo-ovarian serous carcinoma (Figure 1). ${ }^{37,39}$ Nevertheless, tubal involvement including STIC is not found in all cases of serous carcinoma and although clonal relationships between STICs and concurrent highgrade serous carcinomas have been identified in the vast majority of cases, it is still disputable 
whether the STIC is a metastasis of the concurrent carcinoma rather than the primary occurrence. ${ }^{37}$ The same train of thought of ovarian surface epithelium invaginated into cortical inclusion cysts applies to STIC cells that can exfoliate, be shed from the fallopian tube and nest at the ovarian surface, be entrapped in cortical inclusions cysts or nest at the peritoneum.

All together, the idea that all ovarian carcinomas are derived from the same cell has been left and the dualistic model with classification into type I and type II tumors has been more and more adopted. ${ }^{32,40}$ Type I tumors are low-grade serous, endometrioid, clear cell, and mucinous carcinomas, are more often diagnosed in early stage and have a more favorable prognosis than type Il tumors. Potential precursor lesions for type I tumors are endometriosis and borderline tumors. Although relatively genetically stable, the following genes are often mutated in these tumors: KRAS, BRAF, PTEN, PIK3CA, CTNNB1, ARID1A, and PPP2R1A. TP53 mutations are very rare in type I tumors as opposed to type II tumors that almost all harbor TP53 mutations, are genetically instable, and behave aggressively. High-grade serous, undifferentiated carcinomas, and carcinosarcomas are type II tumors and most likely originating from the fallopian tube through STICS. BRCA-related ovarian carcinomas are almost exclusively type $1 .{ }^{32,40}$

Although the fallopian tube is thus particularly associated with type II tumors, the fallopian tube and type I tumors can be linked as well. Low-grade serous carcinomas may arise from papillary tubal hyperplasia; endometrioid and clear cell carcinomas through retrograde menstruation for which the fallopian tubes are needed; and mucinous carcinomas may develop through Walthard cell nests, paratubal cysts and Brenner tumors. ${ }^{41}$

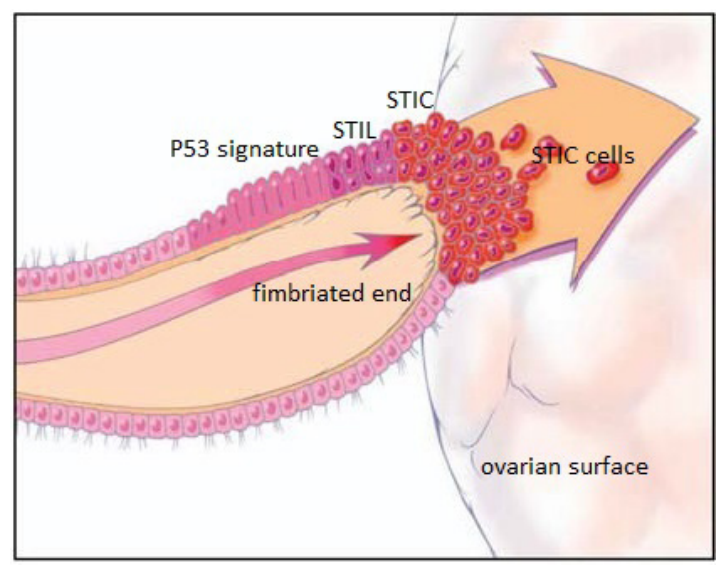

Figure 1. A schematic representation of the hypothesized carcinogenic pathway of high-grade serous carcinoma originating in the fallopian tube. Eventuallly, STIC cells exfoliate and implant on ovarian surface. Abbreviations: STIL serous tubal intraepithelial lesion; STIC serous tubal intraepithelial carcinoma. Figure extracted and edited from: Kurman RJ, Shih IM. The origin and pathogenesis of epithelial ovarian cancer: a proposed unifying theory. Am J Surg Pathol 2010; 34(3): 433-43. ${ }^{79}$ 


\section{Ovarian carcinoma risk management in BRCA1/2 mutation carriers}

Initially, ovarian carcinoma surveillance, consisting of annual transvaginal ultrasonography and measurement of serum level of the tumor marker Cancer Antigen 125 (CA125), was offered to BRCA1/2 mutation carriers. However, both sensitivity and positive predictive value of this surveillance regimen turned out to be low leading to inability to detect early-stage ovarian carcinoma or improve prognosis. ${ }^{42-44}$

Therefore, most international guidelines recommend RRSO to BRCA1/2 mutation carriers from the age of 35 years and when childbearing is completed. 18,41,45,46 Some guidelines distinguish BRCA2 from BRCA1 mutation carriers in terms of recommended age for RRSO: 40-45 years versus 3540 years, respectively. ${ }^{18,46}$ This distinction is based on the risk of ovarian carcinoma increasing at older age in BRCA2 mutation carriers: their cumulative risk to develop ovarian carcinoma before the age of 50 years is only nearly $1 \% .{ }^{47}$ In two meta-analyses, a hazard ratio of about 0.20 was calculated for ovarian carcinoma after RRSO, corresponding with $80 \%$ risk reduction. ${ }^{48,49}$ The best effect ever reported is $96 \%$ risk reduction. ${ }^{50}$ In other words, a residual risk of developing primary peritoneal carcinoma exists but widely varies in literature. This might be due to differences in follow-up time and mean age at RRSO and whether total salpingo-oophorectomy was performed or oophorectomy alone. In the 20 years following RRSO, the risk of primary peritoneal carcinomas is estimated about 3.9\% for BRCA1 mutation carriers and 1.9\% for BRCA2 mutation carriers. ${ }^{51}$ The origin, carcinogenesis, and characteristics of primary peritoneal carcinoma occurring after RRSO are still unclear. RRSO may also effect mortality: a 77\% risk reduction in all-cause mortality to age 70 after RRSO was calculated among BRCA1/2 mutation carriers without a previous cancer diagnosis at the time of RRSO. ${ }^{51}$ RRSO is mostly performed by a laparoscopic procedure and can be done in an outpatient setting. Serious surgical complications occur in only 1-2\%.,52-55

The protocol for Sectioning and Extensively Examining the FIMbriated end (SEE-FIM) was introduced in 2006 in reaction to the detection of dysplastic cells in the fallopian tubes of RRSO specimens in $2001^{38}$ and the subsequent attention that was increasingly paid to the fallopian tubes as the potential site of origin of most ovarian carcinomas. ${ }^{56}$ In this SEE-FIM protocol, the fallopian tube is entirely embedded and examined. Fimbriated ends are sectioned in longitudinal direction and the remainder of the fallopian tube is sectioned at 2- to 3-millimeter intervals. In RRSO specimens of $B R C A 1 / 2$ mutation carriers, occult ovarian carcinomas are detected in approximately $2 \%$ to $9 \%$ and STIC without an invasive component in about 0 to $7 \%$ in recent studies where the SEE-FIM protocol was applied in most cases. Incidence of both occult carcinoma and STIC increases with age. ${ }^{51,54,57-64}$

\section{Premature menopause}

Although RRSO at the currently recommended age is very effective in reducing the risk of developing ovarian carcinoma, acute onset of surgical premature menopause occurs after removal of the ovaries. This sudden estrogen deficiency is responsible for several short-term as well as longterm health consequences on both physical and psychological domains that can significantly 
affect quality of life. However, to what extent quality of life is affected and whether it concerns a temporary or permanent effect has not been crystallized.

Vasomotor symptoms (i.e. hot flashes, night sweats), sleep disturbances, vaginal dryness, sexual dysfunction and mood swings are examples of short-term effects. ${ }^{1,65}$ Probably the most important long-term consequence is the 1.50 (95\% Cl 1.28-1.76) times increased risk of coronary heart disease, 1.19 (95\% Cl 1.08-1.31) times increase of cardiovascular mortality and 1.12 (95\% Cl 1.031.21) times of all-cause mortality for women from the general population who enter menopause before the age of 45 years compared to women older than 45 years. ${ }^{66}$ In another study, the hazard ratio of developing ischemic heart disease was $8.7(95 \% \mathrm{Cl}$ 2.0-38.1) in women who underwent bilateral oophorectomy under age 40 compared to those who underwent the same surgery after age $45 .{ }^{67}$ The risk of cognitive impairment has been found to be about 1.89 (95\% Cl 1.27 2.83) times higher in women who were younger than 49 years at oophorectomy and did not receive hormone replacement therapy (HRT). ${ }^{68}$ Furthermore, increased risks of parkinsonism and depressive and anxiety symptoms have been reported. ${ }^{68,69}$ Lastly, studies on osteoporosis and fracture risk are contradictive: some described higher rates of osteoporosis after premenopausal $\mathrm{RRSO}^{70}$ whereas others reported bone mineral density and fracture incidence comparable to an age-matched reference population..$^{71}$ A third study reported a clear bone loss within 18 months after surgical menopause but lacked a control group. ${ }^{72}$ It must be taken into account that these studies were mostly not performed in the BRCA carrier population specifically, but mainly in the general population. More studies are needed to further elucidate long-term effects of RRSO in $B R C A 1 / 2$ mutation carriers.

HRT is recommended between RRSO and the age of 48-50 years to alleviate climacteric symptoms and prevent long-term consequences, unless HRT is contra-indicated because of a personal history of breast cancer or thrombo-embolic events, for example. HRT partially relieves short-term effects of premature menopause. ${ }^{73-75}$ Evidence for the effect of HRT on long-term consequences is sparse, but it appears beneficial for most of them. ${ }^{67,68,71}$ Although HRT might affect breast cancer incidence in this high-risk population, current evidence suggests no adverse effect on oncologic outcomes in BRCA1/2 mutation carriers without previous breast cancer under the condition that its use is temporary and discontinued at the age of natural menopause. ${ }^{76}$

Non-hormonal treatments for menopausal symptoms consist of selective serotonin reuptake inhibitors (SSRI) and alpha-2 adnergic agonists like clonidine. Body-mind interventions like cognitive-behavioral therapy and mindfulness-based stress reduction have also been shown to reduce the bother of vasomotor symptoms. ${ }^{77,78}$ Furthermore, alternative medicine therapies exist but hardly any research data considering their effectiveness have been published. ${ }^{78}$ 


\section{AIM AMD OUTLIME OF THIS THESIS}

As outlined in the first part of this chapter, two trends have been observed that are relevant to ovarian carcinoma risk management in BRCA1/2 mutation carriers. First, an important role in the development of most, mainly serous, ovarian carcinomas is attributed to the fallopian tubes. Second, evidence is growing on long-term health consequences of premature surgical menopause that may affect quality of life. The combination of those trends led to the proposal of an alternative to RRSO: risk-reducing salpingectomy (RRS) upon completion of childbearing with second-stage oophorectomy delayed beyond the currently recommended age for RRSO. This alternative strategy allows women to retain their ovaries towards the onset of natural menopause and therefore, it might improve their (menopause-related) quality of life. Removal of the fallopian tubes might take place before the currently recommended age for RRSO, depending on family planning, which might prevent (pre)malignant lesions from developing in the fallopian tubes. However, the risk of ovarian carcinoma under this alternative strategy is unknown. Besides, we do not know whether the alternative strategy will be accepted by both BRCA1/2 mutation carriers and healthcare providers. An optimally designed clinical trial is needed to point out efficacy and safety of such a strategy.

\section{Main objectives}

- To evaluate the magnitude of adverse quality of life effects after RRSO

- $\quad$ To gain insight into the number of BRCA1/2 mutation carriers that might face premature menopause due to RRSO in our region

- $\quad$ To explore the possibility of a risk-reducing strategy alternative to RRSO among both BRCA1/2 mutation carriers and professionals: salpingectomy upon completion of childbearing with delayed oophorectomy

- To estimate the effect of the alternative strategy in terms of ovarian carcinoma risks

- To optimally design a clinical trial comparing sal pingectomy with delayed oophorectomy with standard RRSO

- $\quad$ To support BRCA1/2 mutation carriers who face the choice between salpingectomy with delayed oophorectomy and RRSO

- To evaluate clinical and pathological characteristics of peritoneal carcinomatosis occurring after RRSO in BRCA1/2 mutation carriers 


\section{Thesis outline}

In chapter 2, we review the literature on quality of life of unaffected BRCA1/2 mutation carriers and how quality of life is influenced by medical choices made regarding cancer risk management. In chapter 3, we evaluated the number of women who undergo RRSO and at what age they undergo this surgery to get a better perception of how many BRCA1/2 mutation carriers face the consequences of RRSO performed before natural menopause at our institution.

Because RRSO is known to prevent most but not all ovarian carcinomas, we collected international data on BRCA1/2 mutation carriers with ovarian carcinoma (peritoneal carcinomatosis) diagnosed several months or years after RRSO and described them in chapter 4.

We invited both BRCA1/2 mutation carriers and healthcare professionals to discuss the hypothesis of the fallopian tube as central player in ovarian carcinogenesis en how to embed these new insights into risk-reducing interventions. We presented them with the alternative risk-reducing strategy consisting of RRS with delayed oophorectomy and explored barriers and facilitators for the acceptance of this alternative strategy and a trial on this topic in chapter $\mathbf{5}$. The opinions of both BRCA1/2 mutation carriers and healthcare providers in the field are qualitatively studied in this chapter.

Since RRS with delayed oophorectomy has not been clinically studied yet, no data exist on its effect on the risk of ovarian carcinoma. However, an estimation of this risk is needed to properly counsel patients who consider this alternative strategy. These estimates are given in chapter $\mathbf{6}$ for BRCA1 and BRCA2 mutation carriers separately. We modeled the risk of ovarian carcinoma for the alternative strategy at various ages and compared this risk to RRSO using input parameters from literature. We estimated risks for best- and worst-case scenarios.

Chapter $\mathbf{7}$ presents the study protocol for a nationwide preference trial in which the alternative strategy of RRS with delayed oophorectomy will be compared to standard RRSO in premenopausal BRCA1/2 mutation carriers: the TUBA study. Primary outcome parameter is menopause-related quality of life. Healthcare providers are strongly discouraged to randomly perform RRS without close follow-up of its effectiveness and safety.

The development of a patient decision aid is described in chapter 8. Aim of this patient decision aid is to facilitate the complex decision-making process that participants of the TUBA study face: choosing between standard RRSO and RRS with delayed oophorectomy.

Finally, a general discussion on the most important results is provided in chapter 9. Previous chapters are discussed in the light of the latest literature and suggestions and implications for future research are made. 


\section{REFEREMCES}

1. Russo A, Calo V, Bruno L, Rizzo S, Bazan V, Di Fede G. Hereditary ovarian cancer. Crit Rev Oncol Hematol 2009; 69(1): 28-44.

2. Hall JM, Lee MK, Newman B, et al. Linkage of early-onset familial breast cancer to chromosome 17q21. Science (80-) 1990; 250(4988): 1684-9.

3. Wooster R, Bignell G, Lancaster J, et al. Identification of the breast cancer susceptibility gene BRCA2. Nat New Biol 1995; 378(6559): 789-92.

4. Powell SN, Kachnic LA. Roles of BRCA1 and BRCA2 in homologous recombination, DNA replication fidelity and the cellular response to ionizing radiation. Oncogene 2003; 22(37): 5784-91.

5. Nelson HD, Fu R, Goddard K, et al. Risk Assessment, Genetic Counseling, and Genetic Testing for BRCARelated Cancer: Systematic Review to Update the US Preventive Services Task Force Recommendation. Rockville (MD); 2013.

6. Chen S, Parmigiani G. Meta-analysis of BRCA1 and BRCA2 penetrance. J Clin Oncol 2007; 25(11): 1329-33.

7. Vos JR, Hsu L, Brohet RM, et al. Bias Correction Methods Explain Much of the Variation Seen in Breast Cancer Risks of BRCA1/2 Mutation Carriers. J Clin Oncol 2015; 33(23): 2553-62.

8. National Cancer Institute. SEER Stat Fact Sheets: Female Breast Cancer. http://seer.cancer.gov/statfacts/ html/breast.html (accessed 09-23-2016.

9. National Cancer Institute. SEER Stat Fact Sheets: Ovarian Cancer. http://seer.cancer.gov/statfacts/html/ ovary.html (accessed 09-23-2016.

10. Mersch J, Jackson MA, Park M, et al. Cancers associated with BRCA1 and BRCA2 mutations other than breast and ovarian. Cancer 2015; 121(2): 269-75.

11. Arts-de Jong M, de Bock GH, van Asperen CJ, Mourits MJ, de Hullu JA, Kets CM. Germline BRCA1/2 mutation testing is indicated in every patient with epithelial ovarian cancer: A systematic review. Eur $J$ Cancer 2016; 61: 137-45.

12. Pennington KP, Walsh T, Harrell Ml, et al. Germline and somatic mutations in homologous recombination genes predict platinum response and survival in ovarian, fallopian tube, and peritoneal carcinomas. Clin Cancer Res 2014; 20(3): 764-75.

13. George A, Riddell D, Seal S, et al. Implementing rapid, robust, cost-effective, patient-centred, routine genetic testing in ovarian cancer patients. Sci Rep 2016; 6: 29506.

14. Vencken PM, Reitsma W, Kriege M, et al. Outcome of BRCA1- compared with BRCA2-associated ovarian cancer: a nationwide study in the Netherlands. Ann Oncol 2013; 24(8): 2036-42.

15. Bolton KL, Chenevix-Trench G, Goh C, et al. Association between BRCA1 and BRCA2 mutations and survival in women with invasive epithelial ovarian cancer. JAMA : the journal of the American Medical Association 2012; 307(4): 382-90.

16. Zhang S, Royer R, Li S, et al. Frequencies of BRCA1 and BRCA2 mutations among 1,342 unselected patients with invasive ovarian cancer. Gynecol Oncol 2011; 121(2): 353-7.

17. Alsop K, Fereday S, Meldrum C, et al. BRCA mutation frequency and patterns of treatment response in BRCA mutation-positive women with ovarian cancer: a report from the Australian Ovarian Cancer Study Group. J Clin Oncol 2012; 30(21): 2654-63.

18. Integraal Kankercentrum Nederland. Richtlijn Erfelijk en Familiair Ovariumcarcinoom (English: Guideline Hereditary and Familial Ovarian Carcinoma). 06-15-2015. http://www.oncoline.nl/erfelijk-en-familiairovariumcarcinoom (accessed 09-23-2016.

19. Integraal Kankercentrum Nederland. Dutch Cancer Registration. Incidence of epithelial ovarian carcinoma / extra-ovarian ovarian carcinoma / tubal carcinoma. http://www.cijfersoverkanker.nl/ selecties/dataset_1/img5829b5c18b34a (accessed 09-23-2016.

20. Integraal Kankercentrum Nederland. Dutch Cancer Registration. Mortality of ovarian and tubal cancer. http://www.cijfersoverkanker.nl/selecties/dataset_3/img5829b5ef84199 (accessed 09-23-2016. 
21. Zhong Q, Peng HL, Zhao X, Zhang L, Hwang WT. Effects of BRCA1- and BRCA2-related mutations on ovarian and breast cancer survival: a meta-analysis. Clin Cancer Res 2015; 21(1): 211-20.

22. Sun C, Li N, Ding D, et al. The role of BRCA status on the prognosis of patients with epithelial ovarian cancer: a systematic review of the literature with a meta-analysis. PLoS One 2014; 9(5): e95285.

23. Lee JM, Hays JL, Annunziata CM, et al. Phase I/lb study of olaparib and carboplatin in BRCA1 or BRCA2 mutation-associated breast or ovarian cancer with biomarker analyses. J Natl Cancer Inst 2014; 106(6): dju089.

24. Hoogerbrugge N, Jongmans MC. Finding all BRCA pathogenic mutation carriers: best practice models. Eur J Hum Genet 2016; 24 Suppl 1: S19-26.

25. Weren RD, Mensenkamp AR, Simons M, et al. Novel BRCA1 and BRCA2 Tumour Test as Basis for Treatment Decisions and Referral for Genetic Counselling of Patients with Ovarian Carcinomas. Hum Mutat 2016.

26. Zaaijer $\mathrm{LH}$, van Doorn HC, Mourits MJ, et al. Outcome of ovarian cancer after breast cancer in BRCA1 and BRCA2 mutation carriers. Br J Cancer 2016.

27. McCluggage WG. Morphological subtypes of ovarian carcinoma: a review with emphasis on new developments and pathogenesis. Pathology 2011; 43(5): 420-32.

28. Tavassoli FA, Devilee P. Pathology and genetics of tumours of the breast and female genital organs: larc; 2003.

29. Mavaddat N, Barrowdale D, Andrulis IL, et al. Pathology of breast and ovarian cancers among BRCA1 and BRCA2 mutation carriers: results from the Consortium of Investigators of Modifiers of BRCA1/2 (CIMBA). Cancer Epidemiol Biomarkers Prev 2012; 21 (1): 134-47.

30. Silverberg SG. Histopathologic grading of ovarian carcinoma: a review and proposal. Int J Gynecol Pathol 2000; 19(1): 7-15.

31. Kurman RJ, Carcangiu ML, Herrington CS, Young RH. WHO Classification of Tumours of Female Reproductive Organs. WHO Classification of Tumours. 4 ed. Lyon: WHO Press; 2014.

32. Kurman RJ, Shih le M. Molecular pathogenesis and extraovarian origin of epithelial ovarian cancer-shifting the paradigm. Hum Pathol 2011; 42(7): 918-31.

33. Fathalla MF. Incessant ovulation--a factor in ovarian neoplasia? Lancet 1971; 2(7716): 163.

34. Scully RE. Early de novo ovarian cancer and cancer developing in benign ovarian lesions. Int J Gynaecol Obstet 1995; 49 Suppl: S9-15.

35. Pothuri B, Leitao MM, Levine DA, et al. Genetic analysis of the early natural history of epithelial ovarian carcinoma. PLoS One 2010; 5(4): e10358.

36. Lauchlan SC. The secondary Mullerian system. Obstet Gynecol Surv 1972; 27(3): 133-46.

37. Singh N, Gilks CB, Wilkinson N, McCluggage WG. The secondary Mullerian system, field effect, BRCA, and tubal fimbria: our evolving understanding of the origin of tubo-ovarian high-grade serous carcinoma and why assignment of primary site matters. Pathology 2015; 47(5): 423-31.

38. Piek JM, van Diest PJ, Zweemer RP, et al. Dysplastic changes in prophylactically removed Fallopian tubes of women predisposed to developing ovarian cancer. J Pathol 2001; 195(4): 451-6.

39. Visvanathan K, Vang R, Shaw P, et al. Diagnosis of serous tubal intraepithelial carcinoma based on morphologic and immunohistochemical features: a reproducibility study. Am J Surg Pathol 2011; 35(12): 1766-75.

40. Kurman RJ, Shih le M. The Dualistic Model of Ovarian Carcinogenesis: Revisited, Revised, and Expanded. Am J Pathol 2016; 186(4): 733-47.

41. Walker JL, Powell CB, Chen LM, et al. Society of Gynecologic Oncology recommendations for the prevention of ovarian cancer. Cancer 2015.

42. Hermsen BB, Olivier RI, Verheijen RH, et al. No efficacy of annual gynaecological screening in BRCA1/2 mutation carriers; an observational follow-up study. Br J Cancer 2007; 96(9): 1335-42. 
43. Woodward ER, Sleightholme HV, Considine AM, Williamson S, McHugo JM, Cruger DG. Annual surveillance by CA125 and transvaginal ultrasound for ovarian cancer in both high-risk and population risk women is ineffective. BJOG 2007; 114(12): 1500-9.

44. Oei AL, Massuger LF, Bulten J, Ligtenberg MJ, Hoogerbrugge N, de Hullu JA. Surveillance of women at high risk for hereditary ovarian cancer is inefficient. Br J Cancer 2006; 94(6): 814-9.

45. Paluch-Shimon S, Cardoso F, Sessa C, et al. Prevention and screening in BRCA mutation carriers and other breast/ovarian hereditary cancer syndromes: ESMO Clinical Practice Guidelines for cancer prevention and screening. Ann Oncol 2016; 27 (suppl 5): v103-v10.

46. National Comprehensive Cancer Network Clinical Practice Guidelines in Oncology. Genetic/Familial High-Risk Assessment: Breast and Ovarian. 09-19-2016. https://www.nccn.org/professionals/physician_ gls/pdf/genetics_screening.pdf (accessed 09-23-2016.

47. Hartmann LC, Lindor NM. The Role of Risk-Reducing Surgery in Hereditary Breast and Ovarian Cancer. N Engl J Med 2016; 374(5): 454-68.

48. Rebbeck TR, Kauff ND, Domchek SM. Meta-analysis of risk reduction estimates associated with riskreducing salpingo-oophorectomy in BRCA1 or BRCA2 mutation carriers. J Natl Cancer Inst 2009; 101(2): 80-7.

49. Marchetti C, De Felice F, Palaia I, et al. Risk-reducing salpingo-oophorectomy: a meta-analysi S on impact on ovarian cancer risk and all cause mortality in BRCA 1 and BRCA 2 mutation carriers. BMC Womens Health 2014; 14(1): 150.

50. Rebbeck TR, Lynch HT, Neuhausen SL, et al. Prophylactic oophorectomy in carriers of BRCA1 or BRCA2 mutations. N Engl J Med 2002; 346(21): 1616-22.

51. Finch AP, Lubinski J, Moller $P$, et al. Impact of oophorectomy on cancer incidence and mortality in women with a BRCA1 or BRCA2 mutation. J Clin Oncol 2014; 32(15): 1547-53.

52. Kauff ND, Satagopan JM, Robson ME, et al. Risk-reducing salpingo-oophorectomy in women with a BRCA1 or BRCA2 mutation. N Engl J Med 2002; 346(21): 1609-15.

53. Meeuwissen PA, Seynaeve C, Brekelmans CT, Meijers-Heijboer HJ, Klijn JG, Burger CW. Outcome of surveillance and prophylactic salpingo-oophorectomy in asymptomatic women at high risk for ovarian cancer. Gynecol Oncol 2005; $97(2): 476-82$.

54. Manchanda R, Abdelraheim A, Johnson $M$, et al. Outcome of risk-reducing salpingo-oophorectomy in BRCA carriers and women of unknown mutation status. BJOG 2011; 118(7): 814-24.

55. Kenkhuis MJ, de Bock GH, Elferink PO, et al. Short-term surgical outcome and safety of risk reducing salpingo-oophorectomy in BRCA1/2 mutation carriers. Maturitas 2010; 66(3): 310-4.

56. Medeiros F, Muto MG, Lee $Y$, et al. The tubal fimbria is a preferred site for early adenocarcinoma in women with familial ovarian cancer syndrome. Am J Surg Pathol 2006; 30(2): 230-6.

57. Powell CB, Chen LM, McLennan J, et al. Risk-reducing salpingo-oophorectomy (RRSO) in BRCA mutation carriers: experience with a consecutive series of 111 patients using a standardized surgical-pathological protocol. Int J Gynecol Cancer 2011; 21(5): 846-51.

58. Evans DG, Clayton R, Donnai P, Shenton A, Lalloo F. Risk-reducing surgery for ovarian cancer: outcomes in 300 surgeries suggest a low peritoneal primary risk. Eur J Hum Genet 2009; 17(11): 1381-5.

59. Lavie O, Moskoviz MG, Auslender R, et al. Clinical and Pathological Characteristics of Incidental Diagnostic Early Occult Malignancy After Risk-Reducing Salpingo-Oophorectomy in BRCA Mutation Carriers. Int J Gynecol Cancer 2016; 26(2): 233-9.

60. Sherman ME, Piedmonte M, Mai PL, et al. Pathologic findings at risk-reducing salpingo-oophorectomy: primary results from Gynecologic Oncology Group Trial GOG-0199. J Clin Oncol 2014; 32(29): 3275-83.

61. Poon C, Hyde S, Grant P, Newman M, Ireland Jenkin K. Incidence and Characteristics of Unsuspected Neoplasia Discovered in High-Risk Women Undergoing Risk Reductive Bilateral Salpingooophorectomy. Int J Gynecol Cancer 2016; 26(8): 1415-20. 
62. Seidman JD, Krishnan J, Yemelyanova A, Vang R. Incidental Serous Tubal Intraepithelial Carcinoma and Non-Neoplastic Conditions of the Fallopian Tubes in Grossly Normal Adnexa: A Clinicopathologic Study of 388 Completely Embedded Cases. Int J Gynecol Pathol 2016; 35(5): 423-9.

63. Conner JR, Meserve E, Pizer $\mathrm{E}$, et al. Outcome of unexpected adnexal neoplasia discovered during risk reduction salpingo-oophorectomy in women with germ-line BRCA1 or BRCA2 mutations. Gynecol Oncol 2014; 132(2): 280-6.

64. Mingels MJ, Roelofsen T, van der Laak JA, et al. Tubal epithelial lesions in salpingo-oophorectomy specimens of BRCA-mutation carriers and controls. Gynecol Oncol 2012; 127(1): 88-93.

65. Tucker PE, Bulsara MK, Salfinger SG, Tan JJ, Green H, Cohen PA. Prevalence of sexual dysfunction after risk-reducing salpingo-oophorectomy. Gynecol Oncol 2016; 140(1): 95-100.

66. Muka T, Oliver-Williams C, Kunutsor S, et al. Association of Age at Onset of Menopause and Time Since Onset of Menopause With Cardiovascular Outcomes, Intermediate Vascular Traits, and All-Cause Mortality: A Systematic Review and Meta-analysis. JAMA cardiology 2016.

67. Lokkegaard E, Jovanovic Z, Heitmann BL, Keiding N, Ottesen B, Pedersen AT. The association between early menopause and risk of ischaemic heart disease: influence of Hormone Therapy. Maturitas 2006; 53(2): 226-33.

68. Shuster LT, Gostout BS, Grossardt BR, Rocca WA. Prophylactic oophorectomy in premenopausal women and long-term health. Menopause Int 2008; 14(3): 111-6.

69. Rocca WA, Grossardt BR, Geda YE, et al. Long-term risk of depressive and anxiety symptoms after early bilateral oophorectomy. Menopause 2008; 15(6): 1050-9.

70. Garcia C, Lyon L, Conell C, Littell RD, Powell CB. Osteoporosis risk and management in BRCA1 and BRCA2 carriers who undergo risk-reducing salpingo-oophorectomy. Gynecol Oncol 2015; 138(3): 723-6.

71. Fakkert IE, Abma EM, Westrik IG, et al. Bone mineral density and fractures after risk-reducing salpingooophorectomy in women at increased risk for breast and ovarian cancer. Eur J Cancer 2015; 51 (3): 400-8.

72. Hibler EA, Kauderer J, Greene MH, Rodriguez GC, Alberts DS. Bone loss after oophorectomy among high-risk women: an NRG oncology/gynecologic oncology group study. Menopause 2016; 23(11): 122832.

73. Tucker PE, Bulsara MK, Salfinger SG, Tan JJ, Green H, Cohen PA. The effects of pre-operative menopausal status and hormone replacement therapy (HRT) on sexuality and quality of life after risk-reducing salpingo-oophorectomy. Maturitas 2016; 85: 42-8.

74. Madalinska JB, van Beurden M, Bleiker EM, et al. The impact of hormone replacement therapy on menopausal symptoms in younger high-risk women after prophylactic salpingo-oophorectomy. J Clin Oncol 2006; 24(22): 3576-82.

75. Johansen N, Liavaag AH, Tanbo TG, Dahl AA, Pripp AH, Michelsen TM. Sexual activity and functioning after risk-reducing salpingo-oophorectomy: Impact of hormone replacement therapy. Gynecol Oncol 2016; 140(1): 101-6.

76. Marchetti C, ladarola R, Palaia I, et al. Hormone therapy in oophorectomized BRCA1/2 mutation carriers. Menopause 2014; 21 (7): 763-8.

77. Carmody JF, Crawford S, Salmoirago-Blotcher E, Leung K, Churchill L, Olendzki N. Mindfulness training for coping with hot flashes: results of a randomized trial. Menopause 2011; 18(6): 611-20.

78. Nonhormonal management of menopause-associated vasomotor symptoms: 2015 position statement of The North American Menopause Society. Menopause 2015; 22 (11): 1155-72; quiz 73-4.

79. Kurman RJ, Shih le M. The origin and pathogenesis of epithelial ovarian cancer: a proposed unifying theory. Am J Surg Pathol 2010; 34(3): 433-43. 


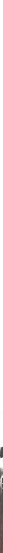

How medical choices influence guality of life of women carrying a BRCA mutation

Marline G. Harmsen, Rosella P.M.G. Hermens, Judith B. Prins, Nicoline Hoogerbrugge, Joanne A. de Hullu 


\section{ABSTRACT}

Germline mutations in $B R C A 1$ and $B R C A 2$ genes were discovered twenty years ago. Female $B R C A$ mutation carriers have an increased risk of breast and ovarian cancer at a relatively young age. Several choices have to be made with respect to cancer risk management, and consequences of these choices may affect quality of life. A review of the literature was performed to evaluate quality of life in unaffected BRCA mutation carriers and the influence of these medical choices. Overall, general quality of life appears not to be permanently affected in BRCA mutation carriers or by their choices. Risk-reducing salpingo-oophorectomy and its subsequent premature menopause affect (menopause specific) quality of life most. Hormone replacement therapy does not fully alleviate climacteric symptoms and therefore, there is a strong need for alternative strategies to reduce ovarian cancer risk and/or for improvements in postoperative care. Future research should focus on these needs. 


\section{InTRODUCTION}

Germline mutations in the two breast cancer (BRCA1/2) genes account for the majority of autosomal dominant inheritance of familial breast and ovarian cancer. ${ }^{1-3}$ Prevalence varies from about $0.2 \%$ in the general population to $20 \%$ in high-risk families. ${ }^{4}$ The mean cumulative risk for breast cancer by age 70 is $57-65 \%$ for BRCA1 and $45-49 \%$ for BRCA2 mutation carriers, compared to approximately $12 \%$ lifetime risk in the general population. ${ }^{2-4}$ Corresponding risks for ovarian cancer are $39 \%$ for BRCA1 mutation carriers and 10-18\% for BRCA2 mutation carriers, whereas lifetime ovarian cancer risk in the general population is about $1.5 \%{ }^{2-4}$ Breast and ovarian cancer in germline BRCA mutation carriers generally occur at younger age than in the general population. ${ }^{1,5,6}$

Female BRCA1/2 carriers have different options to handle their elevated cancer risk. Risk-reducing mastectomy (RRM) with or without breast reconstruction reduces breast cancer risk by $90-100 \%$ although it has not been proven yet to improve survival rates compared to intensive surveillance using mammography, MRI and/or ultrasound. ${ }^{7-10}$ Risk-reducing salpingo-oophorectomy (RRSO) is very effective, reducing ovarian cancer risk by circa $80 \%$ and disease-specific mortality by $79 \% .{ }^{11-13}$ However, removal of the ovaries at the currently recommended age of around 40 years results in acute onset of premature menopause which has short and long-term health consequences. ${ }^{1,14-19}$ Hormone replacement therapy (HRT) has been shown to alleviate most but not all effects of surgical menopause to greater or lesser extent. ${ }^{17,18,20}$ Furthermore, in a substantial part of BRCA mutation carriers HRT is contraindicated because of a breast cancer history.

Women are more tended to choose for RRM and/or RRSO when they are younger, have children, have had breast cancer, have a strong family history, and when they overestimate their cancer risk or have high levels of cancer-related anxiety. ${ }^{21-24}$ All risk-reducing strategies and deciding on them may influence levels of anxiety, psychological distress, body image, sexual functioning, and physical health, ${ }^{25}$ all of which are considered domains contributing to quality of life (QoL). Although universal definitions of QoL lack, the term is often used to describe general health status. ${ }^{26,27}$ By influencing the above mentioned domains, the decision making process, and undergoing interventions to reduce cancer risks might affect QoL. Once a BRCA mutation carrier is diagnosed with cancer, QoL is influenced by the diagnosis "cancer" and by its possible treatments, as is commonly described for (sporadic) breast cancer patients. ${ }^{28,29}$ However, the extensive field of QoL in breast cancer patients and survivors has been left outside the scope of this review in order to focus on unaffected BRCA mutation carriers.

The aim of this review is to evaluate QoL in female BRCA1/2 mutation carriers and to find out to what extent it is influenced by medical decisions and interventions in cancer risk management. We focused on BRCA mutation carriers without a history of breast or ovarian cancer. Knowledge about which factors influence QoL most will be the basis for future research on interventions to optimize QoL. 


\section{METHODS}

\section{Literature search}

We searched Pubmed and the Cochrane database of systematic reviews to identify possible relevant studies regarding QoL in BRCA mutation carriers published between the discovery of BRCA genes in 1993 and the last date of search, September 15 th 2014 . We searched for the following keywords: $B R C A$, hereditary breast and ovarian cancer, $\mathrm{HBOC}$, familial ovarian cancer, familial breast cancer, family ovarian cancer, family breast cancer and quality of life, QoL, health, depression, anxiety, emotional, social, psychosocial, psychological, distress, well being, anxiousness, cancer worry.

\section{Eligibility criteria for studies}

We identified relevant articles by screening titles and abstracts and subsequently by reading the full text. We manually screened reference lists and related articles to find additional relevant papers. We included articles published in English or Dutch, regardless of design. As additional criteria we formulated that at least five BRCA mutation carriers must be part of the study population. Studies were excluded when results of BRCA mutation carriers were indistinguishable from carriers of germline mutations in other genes or when the duration of follow-up and/or the number of (un)affected participants were unclear. Furthermore, studies on both male and female mutation carriers were excluded when data on women alone could not be extracted. To distinguish between the effect on quality of life of being a mutation carrier and the effect on quality of life of the genetic test itself and its disclosure (which is outside the scope of this review), minimal follow-up had to be 12 months after test disclosure. This interval was based on the experience with presymptomatic genetic testing for Huntington's disease: most people appear to return to baseline levels after a temporary increase in depression and anxiety after test disclosure. ${ }^{30}$

\section{Data extraction and comparison}

We designed data extraction tables to systematically extract study characteristics and results from the selected studies, i.e. sample size, participants, design and aim of the study, main reported results relevant to this review and notable limitations and considerations. We clustered results by different aspects of QoL, i.e. general QoL; psychological distress, depression, anxiety, and cancer worry; body image and sexual functioning. For studies including surveillance visits or surgical procedures, QoL after these interventions was compared to baseline. In studies without any intervention, both comparisons over time and between BRCA mutation carriers and the general population were extracted. When applicable, differences between unaffected women and those with a history of (breast) cancer were reported as well. 


\section{RESULTS}

The Pubmed search yielded 514 articles. After applying all eligibility criteria, 33 articles were left to review. Eight articles were about BRCA mutation carriers 12 months after genetic test disclosure; five about QoL during breast cancer surveillance and seven about (considering) RRM. Another ten articles discussed QoL after RRSO with or without the effect of HRT. At last, QoL after both RRM and RRSO was evaluated in three other articles. A limited number of studies including only unaffected female BRCA mutation carriers were available. The majority included women with a personal (breast) cancer history as well and women at high risk for familial breast and ovarian cancer without a documented BRCA mutation. Therefore, specific data on unaffected BRCA mutation carriers were extracted from the original report and shown in this review when possible.

\section{Carrying a germline $B R C A$ mutation}

We identified eight articles about QoL-related items in mainly unaffected women at high risk for hereditary breast and ovarian cancer who were genetically tested for BRCA mutations. All studies were prospective and observational with baseline measurements and follow-up at several time points after testing ${ }^{31-38}$ or focus group participation. ${ }^{38}$ Heterogeneity existed in used QoL questionnaires, with the Impact of Event Scale (IES) and Hospital Anxiety and Depression Scale (HADS) most commonly used. Number of participants varied between 32 and 201 women. Details on each study are shown in table 1.

\section{General QoL}

Little is reported about general QoL. Lower QoL was found in BRCA mutation carriers whose mother had deceased compared to BRCA mutation carriers whose mother was still alive, with FACT-G scores of about 75 versus 98 respectively (p0.003). ${ }^{38}$ 


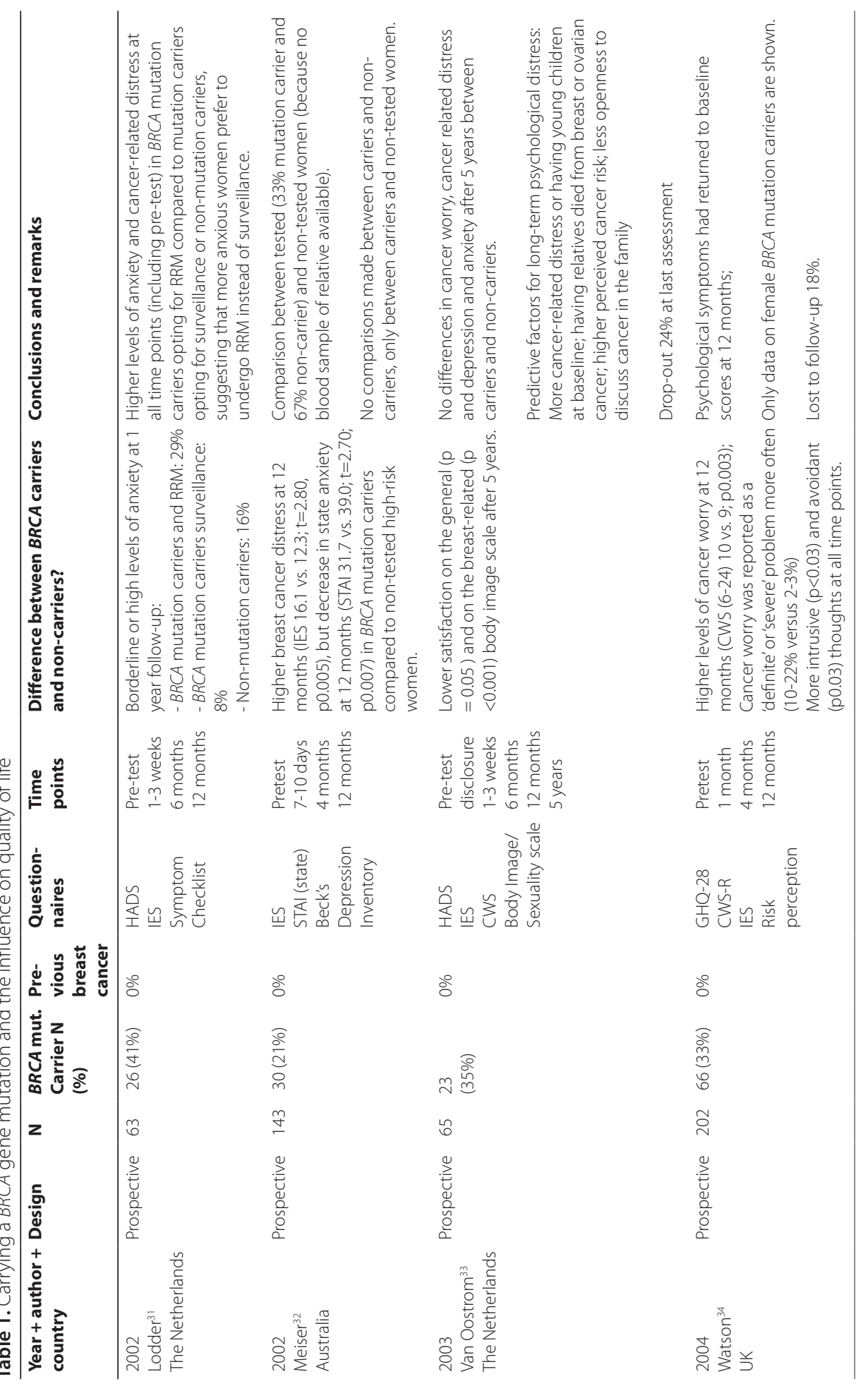




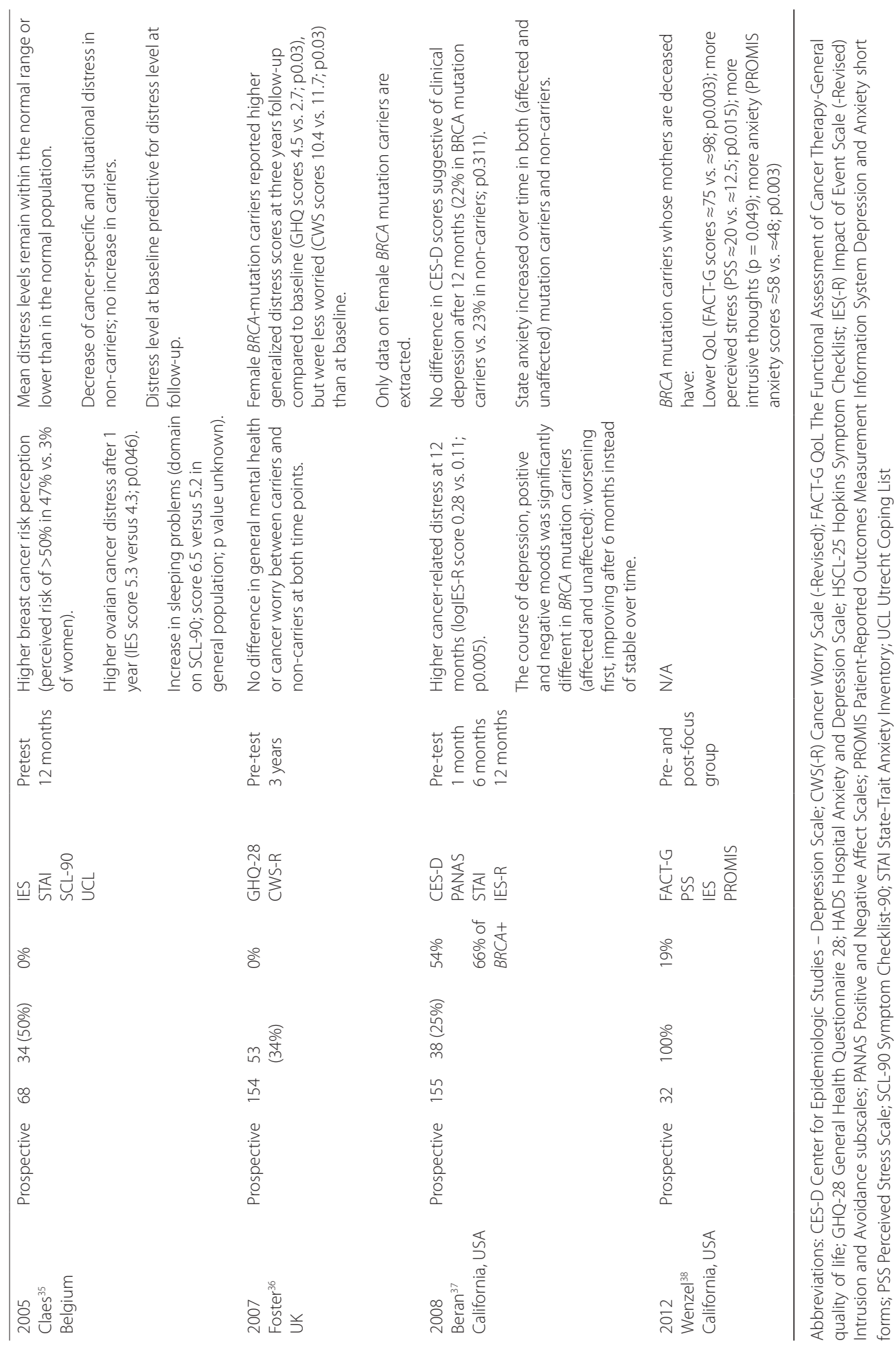


Psychological distress: anxiety, depression and cancer worry

Compared to women whose genetic test was negative, BRCA mutation carriers had significantly more definite or severe cancer worries (10-22\% vs. 2-3\%) and cancer related distress (IES 5.3-16.1 vs. 4.3-12.3 and logIES-R score 0.28 vs. 0.11) after one year, 32,34,35,37 but the difference in cancer worry was not found at 3 and 5 years after testing. ${ }^{33,36}$ For general distress and general mental health, no statistically significant differences were found between carriers and non-carriers. ${ }^{35,36}$ Nevertheless, BRCA mutation carriers had more sleeping problems (as a domain of SCL-90: 6.5 vs. 5.2 in the general population and 'less' in non-mutation carriers) ${ }^{35}$ and higher cancer risk perception after one year (47\% of BRCA mutation carriers vs. 3\% of non-carriers perceived their breast cancer risk more than 50\%). ${ }^{35}$ Psychological symptoms like anxiety and depression were at or below baseline levels after one year.,32,34,37 Long-term psychological distress was predicted by baseline values, including more distress at baseline, ${ }^{33,35}$ having young children, ${ }^{33}$ higher perceived cancer risk, ${ }^{33}$ and cancer deaths in the family. ${ }^{33,38}$ Women who had higher levels of anxiety and cancer worry at baseline were more likely to opt for risk-reducing surgery. ${ }^{31}$

Body image and sexual functioning

$B R C A$ mutation carriers reported lower satisfaction rates on general and breast-related body image after 5 years ( $\mathrm{p} 0.05$ and $\mathrm{p}<0.001$ respectively; no effect sizes available). ${ }^{33}$

\section{Breast cancer in germline $B R C A$ mutation carriers}

\section{Surveillance}

We identified five relevant articles about the influence of surveillance for breast cancer on QoL or related items. ${ }^{39-43}$ There were no randomized controlled trials in literature. All studies were prospective, included 55 to 357 women at high risk for breast and ovarian cancer, and the absolute number of BRCA mutation carriers varied from 17 to 55 women. Only three studies were limited to unaffected women, 39,40 while $3 \%$ and $58 \%$ of the participants had a personal history of breast or ovarian cancer in the other two. ${ }^{41,42}$ Authors used different questionnaires at different time points, but at least before a surveillance visit and 1 month until 5-8 years thereafter. Details on each study can be found in table 2 .

\section{General QoL}

Rijnsburger et al..$^{39}$ found significantly higher QoL scores in high-risk women under surveillance than in the general population on validated QoL questionnaires (SF-36 physical subscale 52.5 versus 50.0; EQ-5D utility 0.88 versus 0.85 ) and these scores did not change over time. However, they reported significantly lower scores on the EQ-5D Visual Analogue Scale (VAS) (81.9 versus 86.9) suggesting lower QoL than in the general population, with a temporary decrease on the day of visit (79.0 versus 81.9 before the visit). ${ }^{39}$ Spiegel et al. reported no differences in QoL scores in women who were recalled after a surveillance visit compared to women who were not. ${ }^{42}$ 
Psychological distress: anxiety, depression and cancer worry

In high-risk women adhering breast cancer surveillance, no changes in distress scores ${ }^{41}$ and anxiety and depression scores ${ }^{43}$ over time were found by some, whereas others described significantly higher cancer distress before the first visit (but within normal ranges, IES 9.6 versus 7.3 thereafter) ${ }^{40}$ and a significant decrease of general distress (IES 7.9 to 6.8 and 7.3 to 6.6$)^{40}$ after surveillance visits. Recall visits after abnormal MRI results significantly increased levels of anxiety at 4-6 weeks after MRI compared to baseline (from HADS-Anxiety 7.8 (SD 4.7) to 8.8 (SD 5.2)) and breast cancer worry compared to non-recalled women (in 35\% vs. 13\% after 6 months), affecting mood in $6 \%$ and $0 \%$ respectively. ${ }^{42}$ Another study found increased avoidance scores after abnormal MRI results compared to baseline (IES avoidance subscale from 6.5 (SD 7.6) to 9.5 (SD 8.4)), but this was found to be related to the overrepresentation of BRCA mutation carriers in this group rather than to MRI results. BRCA mutation carriers showed greater increase of distress scores between a first and second MRI versus BRCA negative or untested subjects (IES increase of 11.6 versus decrease of 0.86 points) and carrying a BRCA mutation was the only predictor for increased distress levels. ${ }^{41}$

After long term adherence to breast cancer surveillance programs, intrusion and avoidance scores decreased (IES-Intrusion from 4.58 (SD 6.12) to 2.75 (SD 4.58) and IES-Avoidance from 4.07 (SD 6.01 ) to 3.34 (SD 6.41)). Identified risk factors for higher distress levels were the loss of a first-degree relative to breast cancer, a passive or palliative coping strategy, risk overestimation and excessive breast self examination. Having a partner and coping through reassuring thoughts were predictors for lower distress levels. ${ }^{43}$

\section{Risk-reducing mastectomy (RRM)}

The only study about QoL in 56 women with increased risk of breast cancer considering prophylactic mastectomy is from Brandberg et al. ${ }^{44}$ Twenty-six were BRCA mutation carriers. Within two years prior to undergoing RRM, women's concerns and expectations of an RRM were evaluated. In the study group, 30\% reported anxiety and 7\% depression above the'normal range', without statistically significant differences between subjects with and without previous breast cancer and between the study group and existing data in the general population.

Six studies reported about QoL and related items like psychological symptoms, body image, sexuality and satisfaction after RRM. ${ }^{45-50}$ A cross-sectional design with measurements after a mean of 42 months $^{48}$ and 52 months ${ }^{45,46}$ after RRM was used in three of them. The other three were prospective studies with baseline measurements before RRM and follow-up until 1 to 6-9 years after RRM. ${ }^{47,49,50}$ Number of included BRCA mutation carriers varied from 13 to 50 in study populations existing of 36 to 90 women at high risk for breast cancer. Four reports included unaffected women only, ${ }^{45-47,50}$ whereas the other two included 36\% and 51\% participants with a personal history of breast cancer. ${ }^{48,49}$ Various questionnaires were used. Study details are shown in table 2. 


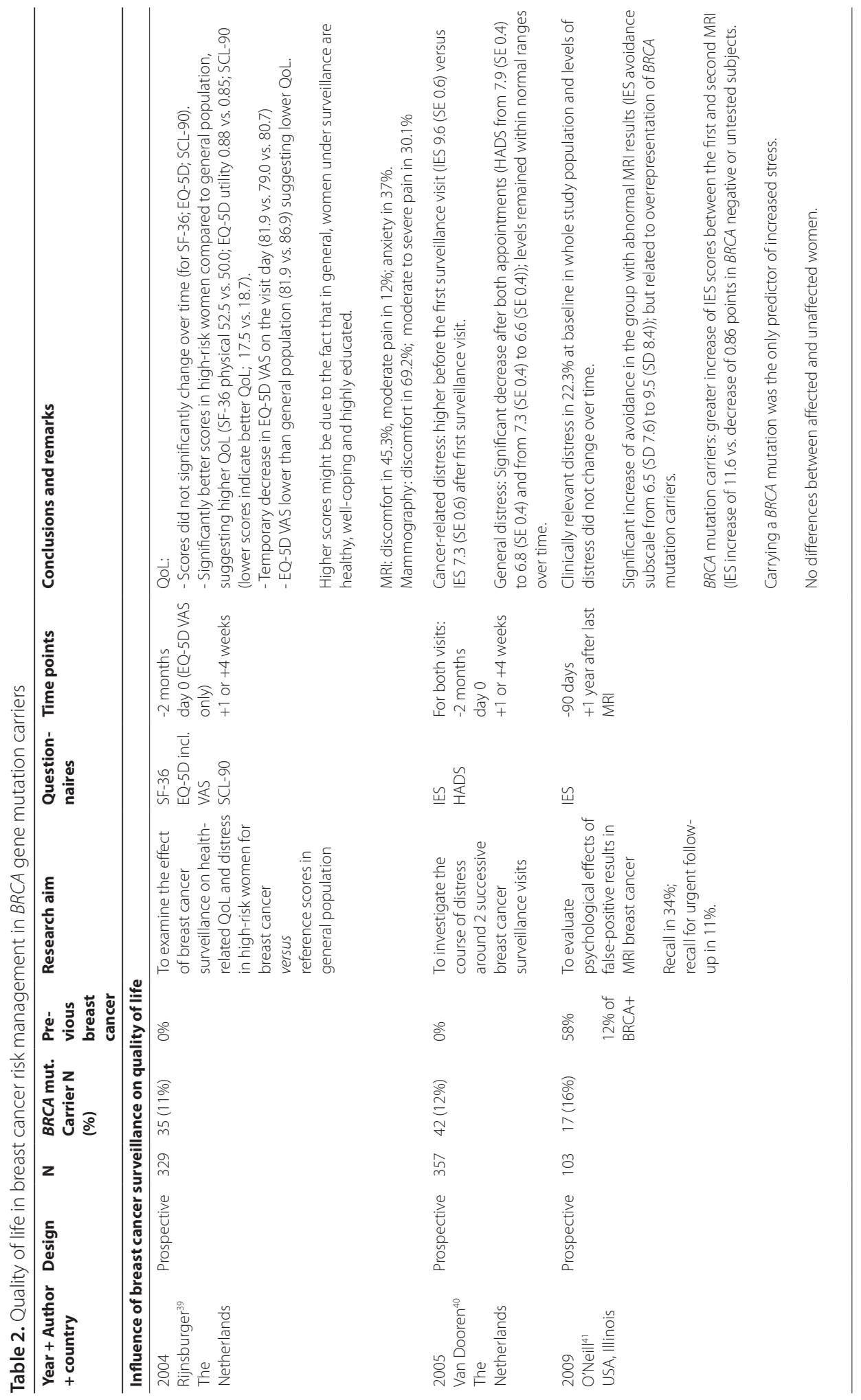




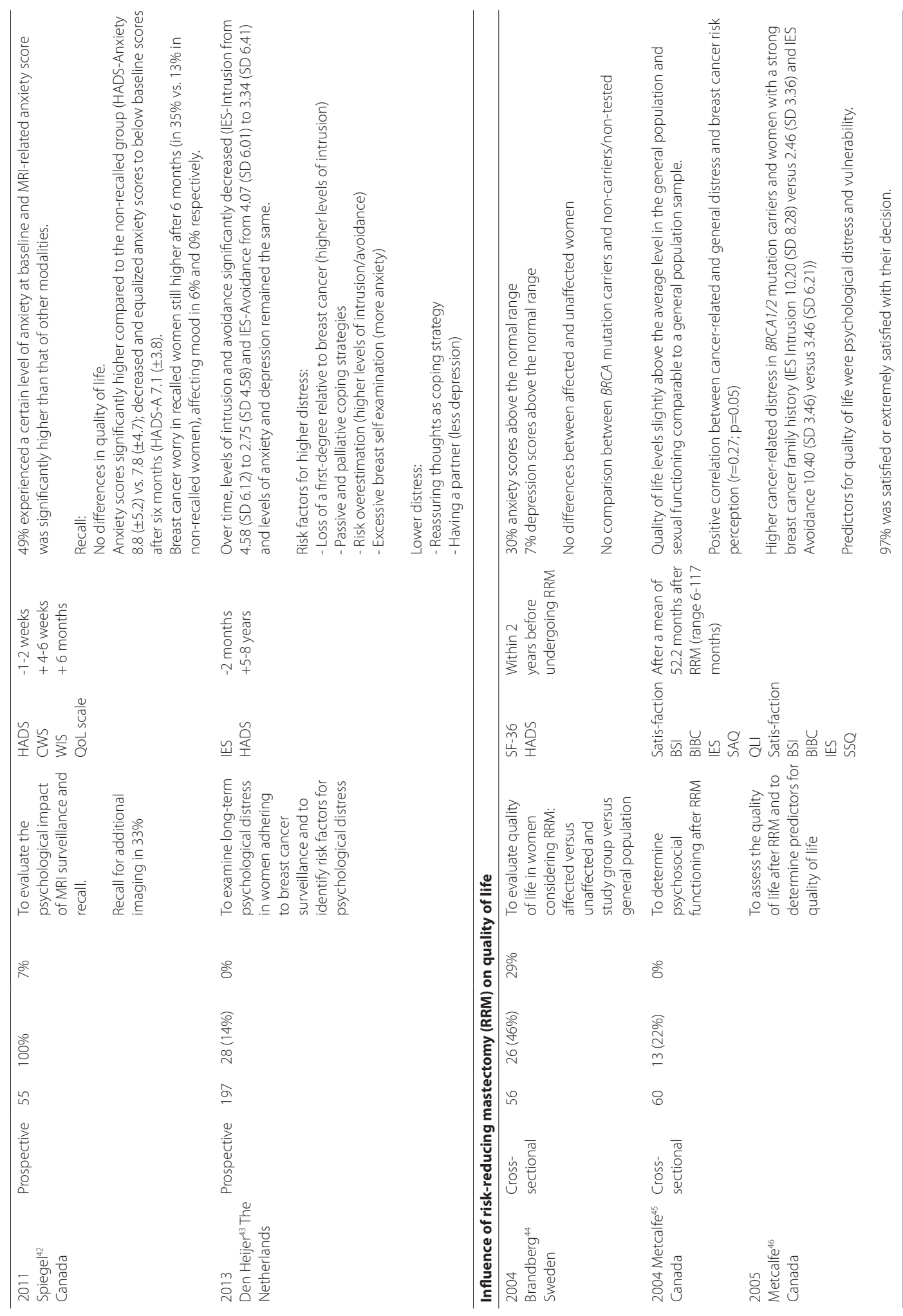




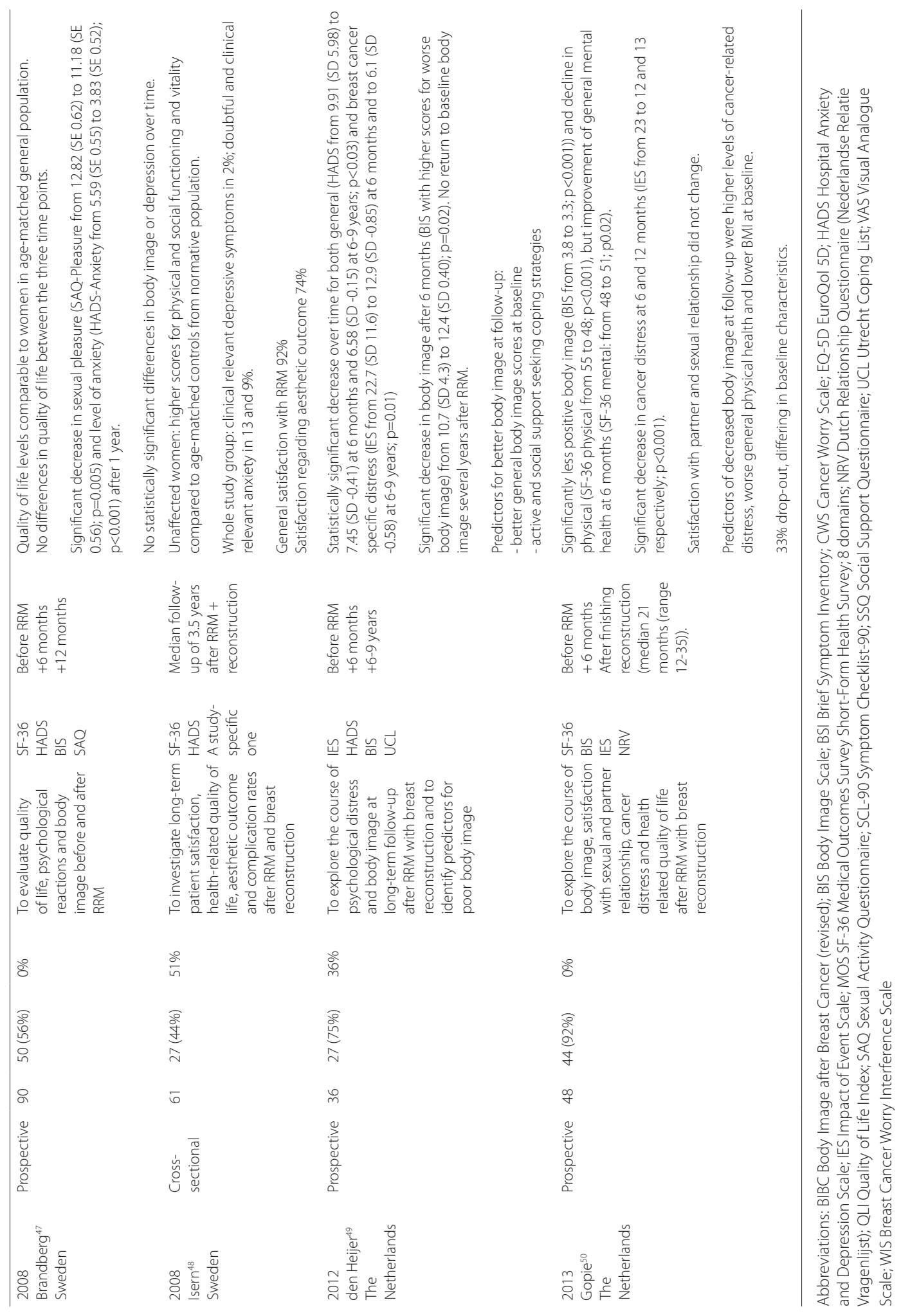




\section{General QoL}

Women's QoL scores after RRM were comparable or even above levels of age-matched women in the general population ${ }^{46,47}$ especially for women without previous breast cancer. ${ }^{48}$ Gopie et al. found a statistically significant improvement in general mental health (SF-36 mental: from 48 to 51; $\mathrm{p}=0.02$ ) and a statistically significant decline in physical health (SF-36 physical from 55 to 48; $\mathrm{p}<0.001)$ at six months after RRM compared to baseline. ${ }^{50}$ In contrast, Brandberg et al. did not find a change in QoL scores after RRM compared to baseline.47 Predictors for QoL were psychological distress and vulnerability. ${ }^{46}$

\section{Psychological distress: anxiety, depression and cancer worry}

General distress decreased after RRM (HADS from 9.91 (SD 5.98) to 7.45 (SD -0.41) at 6 months and to 6.58 (SD -0.15) at 6-9 years; $P<0.03$ ), as did cancer-related distress (IES from 23 at baseline to 12 at 6 months and to 13 at 12 months; $p<0.001$ and IES from 22.7 (SD 11.6) to 12.9 (SD -0.85) at 6 months and to 6.1 (SD -0.58) at 6-9 years; $p=0.01$ ). ${ }^{49,50}$ Cancer-related distress was found to be significantly higher in BRCA mutation carriers and in women with a strong family history of breast cancer compared to women without a BRCA mutation or strong family history (IES Intrusion 10.20 (SD 8.28) versus 2.46 (SD 3.36) and IES Avoidance 10.40 (SD 3.46) versus 3.46 (SD 6.21)). ${ }^{45}$ Anxiety levels were decreased one year after RRM (HADS-Anxiety from 5.59 (SE 0.55) to 3.83 (SE $0.52) ; p<0.001$ ), whereas the prevalence of depressive symptoms did not significantly change.47

\section{Body image and sexual functioning}

On sexual functioning, a decrease in the pleasure subscale was observed one year after RRM (Sexual Activity Questionnaire-Pleasure from 12.82 (SE 0.62) to 11.18 (SE 0.56); $p=0.005$ ). ${ }^{47}$ However, the general level of sexual functioning after RRM was comparable to the general population ${ }^{45}$ and satisfaction with sexual and partner relationship did not change from baseline. ${ }^{50}$ Body image did not change after RRM in one study, ${ }^{47}$ but was less positive at 6 months after RRM in two other studies (Body Image Scale (higher scores for worse body image) from 10.7 (SD 4.3) to 12.4 (SD 0.40); $\mathrm{p}=0.02$ and from 3.8 to 3.3 on a study specific body image scale with higher scores for better body image; $\mathrm{p}<0.001),{ }^{49,50}$ which was predicted by higher cancer distress, lower general physical health and lower body mass index at baseline..$^{50}$ Better body image was predicted by better body image at baseline and active and support seeking coping strategies. ${ }^{49}$

Women were highly satisfied with RRM in general (92-97\%) ${ }^{45,48}$ and with aesthetic outcome (74\%).48 However, satisfaction is hard to measure objectively, since women will unconsciously enable everything to justify the previously made decision to reduce cognitive dissonance. ${ }^{51}$ 


\section{Ovarian cancer in germline $B R C A$ mutation carriers}

Our literature search yielded eight articles concerning QoL of BRCA mutation carriers after RRSO, of which three were prospective with measurements before and until 12 months after surgery ${ }^{20,52,53}$ and five cross-sectional ${ }^{54-58}$ of which one also retrospectively evaluated the presurgical status, ${ }^{57}$ with mean follow-up durations of 6 months to 6 years. Number of subjects per study varied from 75 to 846 women at high risk for breast and ovarian carcinoma with 26 to 364 BRCA mutation carriers amongst them. Three studies exclusively included BRCA mutation carriers. ${ }^{20,53,58}$ No studies included unaffected women only, resulting in 20-77\% women with a personal history of breast cancer in each study. The proportion of women who were premenopausal at the time of RRSO varied between 38-75\%. Different questionnaires were used, mainly evaluating (menopauserelated) QoL, sexual functioning and psychological symptoms. Details of included studies can be found in table 3 .

\section{General QoL}

Levels of generic QoL after RRSO were generally high and equal to women adhering gynecologic surveillance and to reference populations (SF-12 Physical Component Summary 50.86 vs. 50 (population norm) and Mental Component Summary 47.41 vs. 50 (population norm); EORTCQLQ-C30 global health score 74.9 (SD 19.0) vs. 76.1 (SD 19.4) in gynecologic surveillance and 68 (SD 20.8) versus about 62 in EORTC standard). 53,54,57 Only the physical component of QoL was found to be temporarily lower at 1 month after surgery compared to women in a surveillance group (Physical Component Summary $\beta-5.61 ; p<0.02$ ), but this difference was no more apparent after 6 and 12 months. ${ }^{52}$ Women who were younger at the time of RRSO, had lower levels of social functioning at follow-up. ${ }^{57}$ QoL was significantly correlated with activity in sports, a better physical condition, absence of weight gain and lower levels of depression. ${ }^{56,57}$ The observation that QoL parameters did not differ between BRCA mutation carriers and high-risk non-mutation carriers was explicitly mentioned in two reports. ${ }^{5,57}$

\section{Menopause-specific QoL}

More endocrine symptoms were reported after RRSO compared to women adhering to surveillance (FACT-ES 56.0 (SD 9.5) vs. 59.7 (SD 9.6); p<0.001; lower scores indicating more endocrine symptoms). ${ }^{54}$ Vasomotor symptoms (e.g. hot flashes) and vaginal dryness were most frequently reported: in $25 \%$ and $47 \%$ respectively ${ }^{57}$ and more than in women adhering to surveillance (55.3\% versus $16.2 \%$ for hot flashes and $44.7 \%$ versus $10.8 \%$ for vaginal dryness after 6 months); however, these differences already existed at baseline in this particular study. ${ }^{52}$ Severe menopausal symptoms were more persisting when RRSO was performed at younger age (correlation $R^{2}=0.878 ; p=0.002$ ). ${ }^{58}$ 

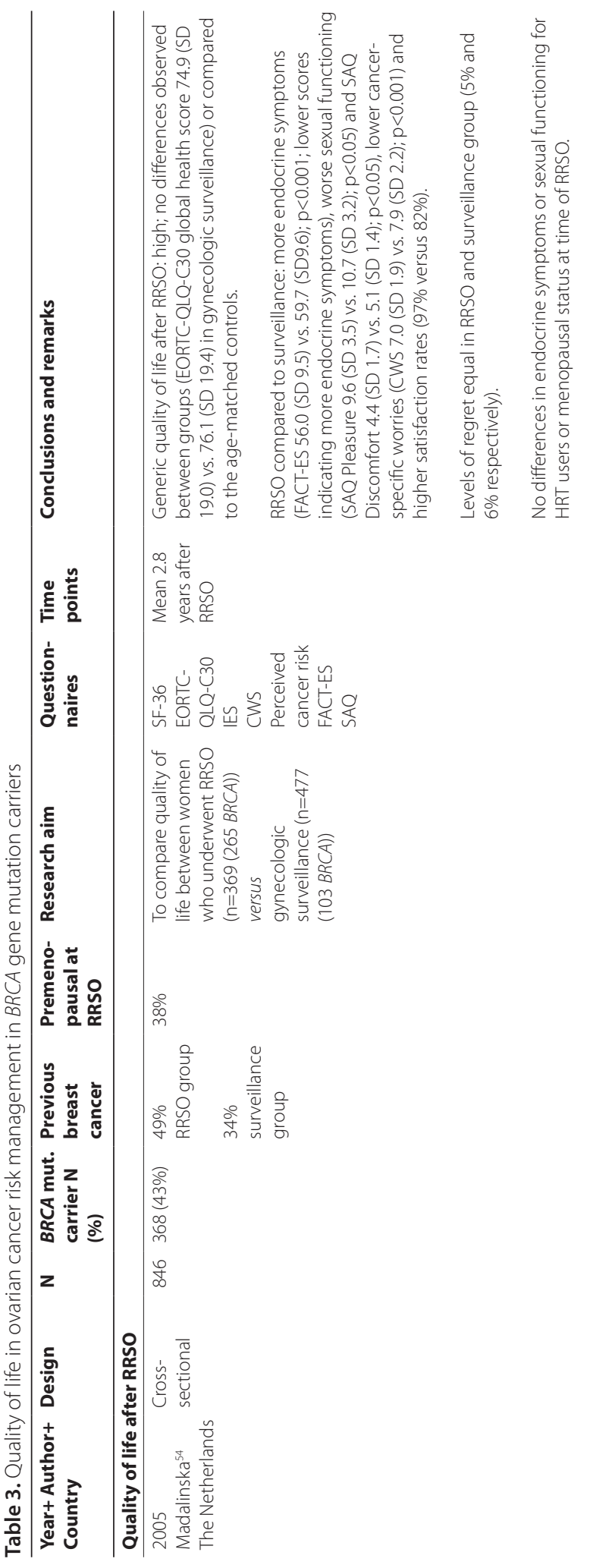

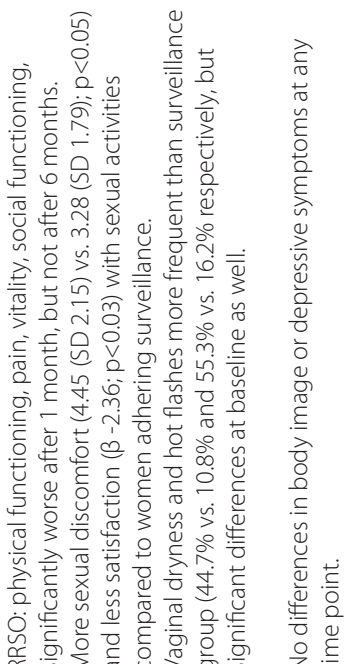

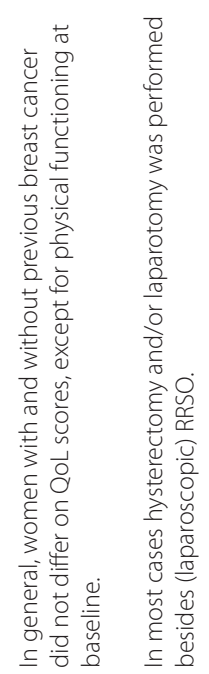

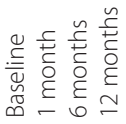

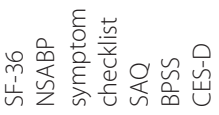

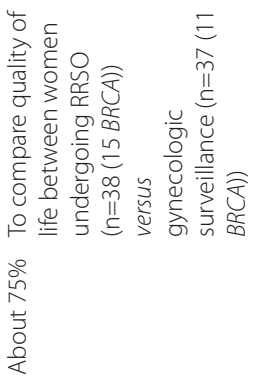

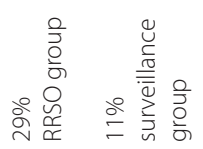

o
in
$\stackrel{0}{0}$
N

농

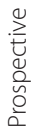

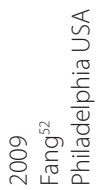




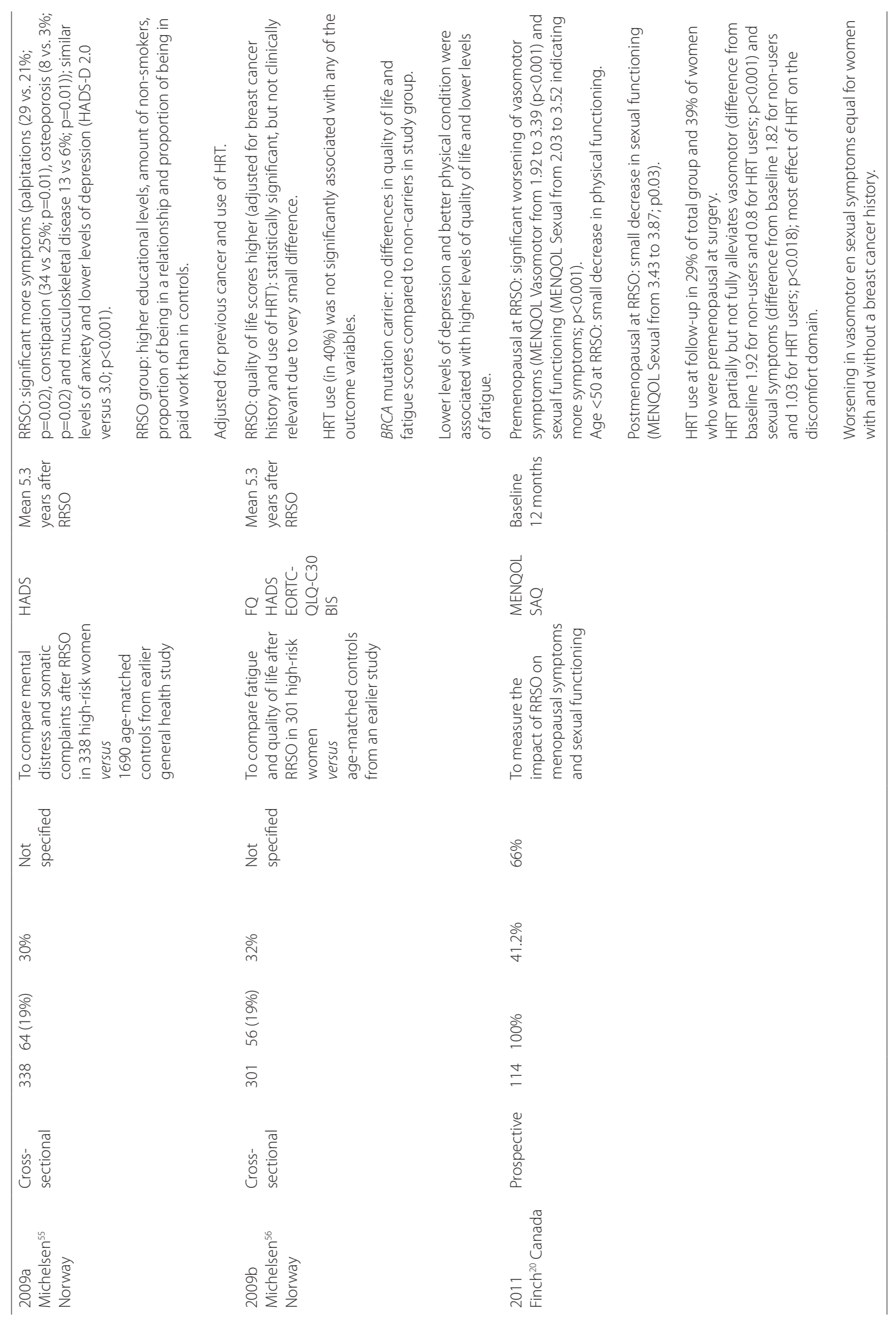




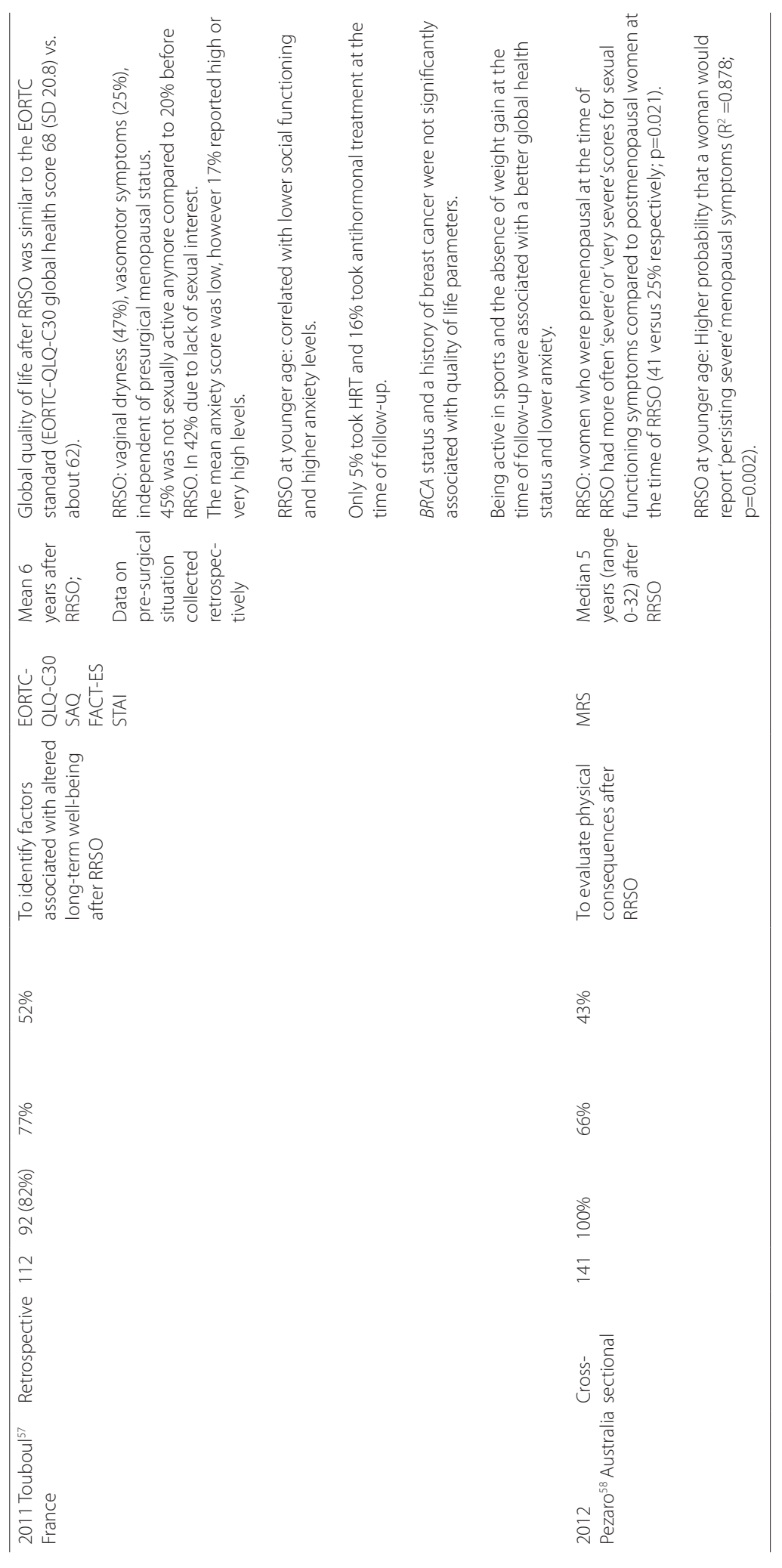




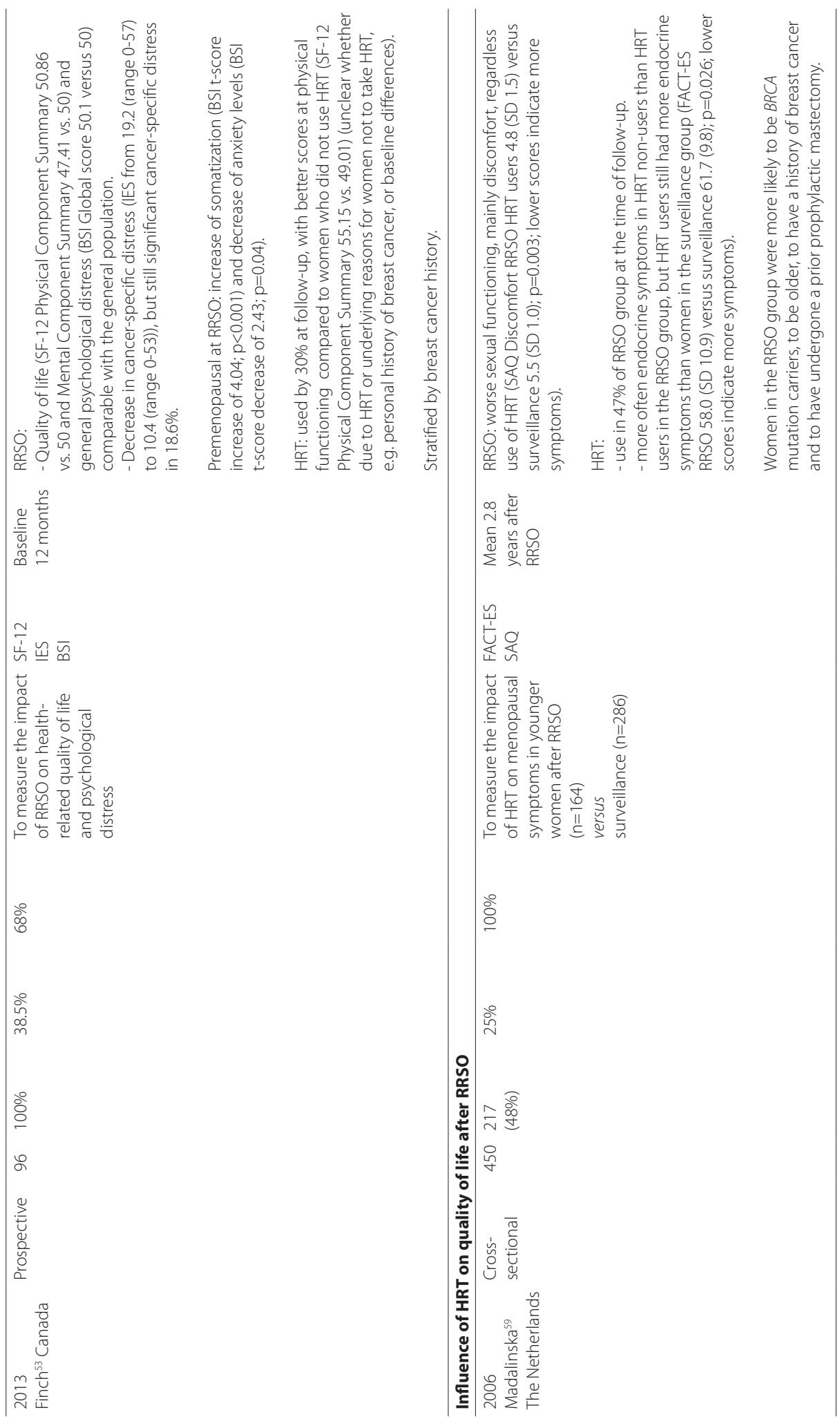




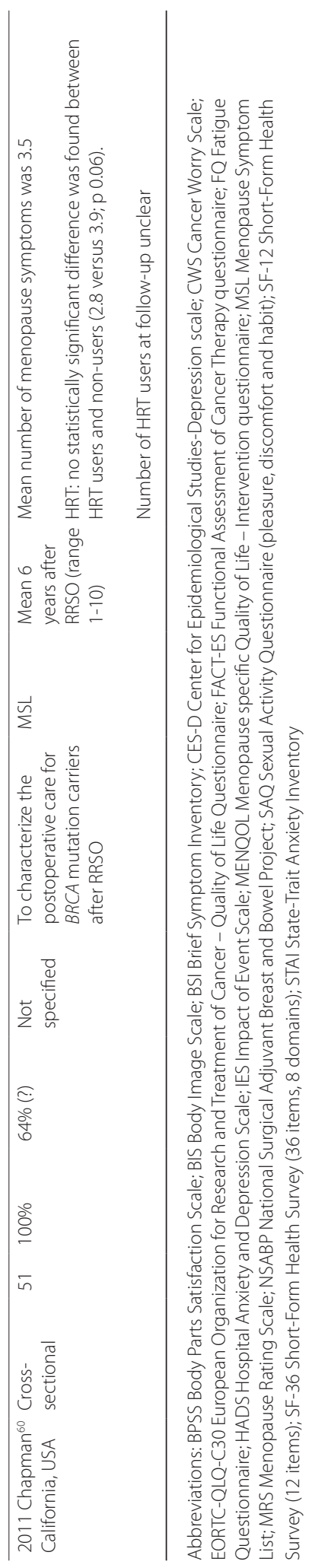


Psychological distress: anxiety, depression and cancer worry

General distress was comparable in women after RRSO compared to age-matched controls from the general population (Brief Symptom Inventory Global score 50.1 versus 50)..$^{53}$ Cancer-specific distress significantly decreased (IES from 19.2 (range 0-57) to 10.4 (range 0-53)) after RRSO but was still moderately or severely present in $19 \%$ of participants. ${ }^{53}$

Levels of anxiety after RRSO were found to be equal to age-matched controls from the general population ${ }^{55}$ with mean anxiety levels being generally low (STAI-A 41.2 (range 20-73)), but high or very high in $17 \% .{ }^{57}$ Being active in sports and absence of weight gain were correlated with lower anxiety levels at follow-up, whereas younger age at RRSO was associated with higher anxiety levels ${ }^{57}$. Finch et al. observed a decrease in anxiety scores (BSI t-score decrease of 2.43; $p=0.04$ ) and increase of somatization (BSI t-score increase of 4.04; $p<0.001$ ) after RRSO in women who were premenopausal (and thus relatively young) at the time of surgery. ${ }^{53}$

Furthermore, cancer worry was less (Cancer Worry Scale 7.0 (SD 1.9) versus 7.9 (SD 2.2); $p<0.001$ ) and satisfaction with preventive strategy was higher (97\% versus $82 \%$ ) after RRSO compared to women adhering to gynecologic surveillance. However, levels of regret were equal (5\% and 6\% respectively). ${ }^{54}$ Levels of depression were found to be equal for RRSO and gynecologic surveillance ${ }^{52}$ and lower after RRSO compared to age-matched controls from the general population (HADS-D 2.0 versus $3.0 ; \mathrm{p}<0.001) .{ }^{55}$

\section{Body image and sexual functioning}

Sexual functioning was worse after RRSO compared to women adhering to surveillance with more discomfort and less pleasure despite use of HRT after RRSO in 37\% of participants (SAQ Pleasure 9.6 (SD 3.5) vs. 10.7 (SD 3.2); $p<0.05$ ) and SAQ Discomfort 4.4 (SD 1.7) vs. 5.1 (SD 1.4); $p<0.05$ ). ${ }^{54}$ Frequency of sexual encounters was temporarily decreased in $67 \%$ of women 1 month after RRSO compared to baseline but then returned to its usual level. ${ }^{52}$ In women who were premenopausal at the time of RRSO, a decrease in sexual functioning was observed compared to baseline although 39\% used HRT (SAQ Pleasure from 13.48 at baseline to 11.35 at follow-up; $p<0.001$, SAQ Discomfort from 4.77 to $3.51 ; p<0.001$ and SAQ Habit from 0.85 to $0.62 ; p=0.04) .{ }^{20}$ More severe or very severe sexual symptoms were reported in premenopausal women than in women who were yet postmenopausal at the time of RRSO (41 versus $25 \%$ respectively; $\mathrm{p}=0.021$ ). ${ }^{58}$ However, the latter group still showed a small decrease in sexual functioning compared to baseline (MENQOL Sexual 3.43 to 3.87 (indicating more symptoms); p0.03)..$^{20}$ At last, 55\% was sexually active after RRSO (mean age 48, range 35-74); sexual inactivity was mostly due to the lack of sexual interest in $42 \% .{ }^{57}$ Differences in sexual functioning between pre- and postmenopausal women at time of RRSO were not confirmed by another study. ${ }^{54}$ Body image levels were similar for women after RRSO and women under gynecologic surveillance. ${ }^{52}$ 
Influence of HRT on QoL after RRSO

In three from the above mentioned studies, a comparison was made between HRT users and nonusers. 20,53,56 Finch et al. found fewer vasomotor symptoms (MENQOL Vasomotor 2.37 versus 4.07; $p<0.001$ ) and better sexual functioning (MENQOL Sexual 4.02 versus 2.80; p0.015) in HRT users compared to non-users with the most significant effect of HRT on discomfort; however, HRT users still had statistically significantly more vasomotor and sexual symptoms compared to baseline (2.37 versus $1.57 ; p<0.001$ and 2.80 versus $1.77 ; p=0.004$ ). ${ }^{20} \mathrm{HRT}$ users also scored better on physical functioning (SF-12 Physical Component Summary 55.15 vs. 49.01), however it was unclear whether this difference was due to HRT, to baseline differences or to underlying reasons for women not to take HRT, e.g. personal history of breast cancer. ${ }^{53}$ In the study by Michelsen et al., HRT could not be significantly associated with any of the outcome variables..$^{56}$ Two other cross-sectional studies focused on menopausal symptoms after almost 3 and 6 years after RRSO (see table 3 for details). ${ }^{59,60}$ Madalinska et al. included 450 women at high risk for hereditary breast and ovarian cancer who were premenopausal at RRSO. ${ }^{59}$ About half of respondents were BRCA mutation carriers and about one third had undergone RRSO. Compared to women choosing for gynecologic surveillance, HRT users in the RRSO group reported more sexual discomfort (SAQ Discomfort RRSO 4.8 (SD 1.5) versus surveillance 5.5 (SD 1.0); $\mathrm{p}=0.003$; lower scores indicate more symptoms) and more endocrine symptoms (FACT-ES RRSO 58.0 (SD 10.9) versus surveillance 61.7 (9.8); $p=0.026$; lower scores indicate more symptoms). HRT users had significantly less endocrine symptoms than women who did not use HRT after RRSO (FACT-ES 58.0 (SD 10.9) for HRT versus 54.6 (SD 9.7) for non-users; p0.034; lower scores indicate more symptoms). Chapman et al. did not find a statistically significant effect for HRT in 51 BRCA mutation carriers with respect to the number of menopause symptoms $(p=0.06)$. However, it is unclear whether participants were pre- or postmenopausal at the time of RRSO and how many used HRT at the time of follow-up (mean follow-up 6 years after RRSO). ${ }^{60}$

\section{QoL after both RRM and RRSO}

Three articles about psychosocial functioning after RRM, RRSO or both were identified (see table 4 for details). Two of these were prospective, from the same author, and included largely the same study population (78 and 82 inclusions with 54 and 58 BRCA mutation carriers respectively). ${ }^{61,62}$ About one third had previous cancer. Questionnaires about general and cancer-specific distress were filled in before risk-reducing surgery and at 6 and 12 months after surgery. The third study had a cross-sectional design including forty only unaffected women at high risk for hereditary breast and ovarian cancer, and used semi-structured interviews at a mean of 3 years after risk-reducing surgery. ${ }^{63}$ Twenty-five were BRCA mutation carriers.

One year after RRM, levels of intrusion (IES from 11.6 (SD 9.3) to 7.2 (SD 7.2); $\mathrm{p}<0.001$ ), avoidance (IES from 10.3 (SD 8.8) to 5.6 (SD 7.0); p<0.001) and anxiety (HADS-A from 7.0 (SD 4.5) to 4.5 (SD 3.1); $p<0.001$ ) all decreased from baseline, ${ }^{61}$ but unanticipated bodily sensations were present in about half of women after RRM and in some cases sexuality was permanently affected (no quantitive 
data). ${ }^{63}$ After RRSO, no significant changes in intrusion, avoidance or anxiety were found in the prospective study, ${ }^{61}$ whereas women in the cross-sectional study reported emotional, sensational and physical impact of menopause and its effect on sexuality the most important changes (no quantitive data). ${ }^{63}$

After risk-reducing surgery in general, cancer worry and cancer-related and general distress decreased (IES from 20.3 (SD 15.2) to 13.1 (SD 11.8); p0.000 and HADS from 10.0 (7.3) to 7.8 (5.4); p0.000).62,63 However, negative emotions were present in half of the respondents either attributed to loss of their breasts or to onset of menopause. ${ }^{63}$

Higher levels of distress at baseline were present in women who opt for risk-reducing surgery instead of surveillance (HADS 10.1 vs. 7.7; $p<0.02$ and IES 20.0 vs. 9.6; $p<0.001$ ) ${ }^{61}$ and were significantly correlated with higher levels of distress at follow-up. ${ }^{62}$ Carrying a BRCA germline mutation was predictive for higher cancer-related distress at 6 months ( $B$ 5.89; $p=0.03$ ), but for lower general distress at 12 months after risk-reducing surgery $(B-3.53 ; p=0.001){ }^{62}$ 


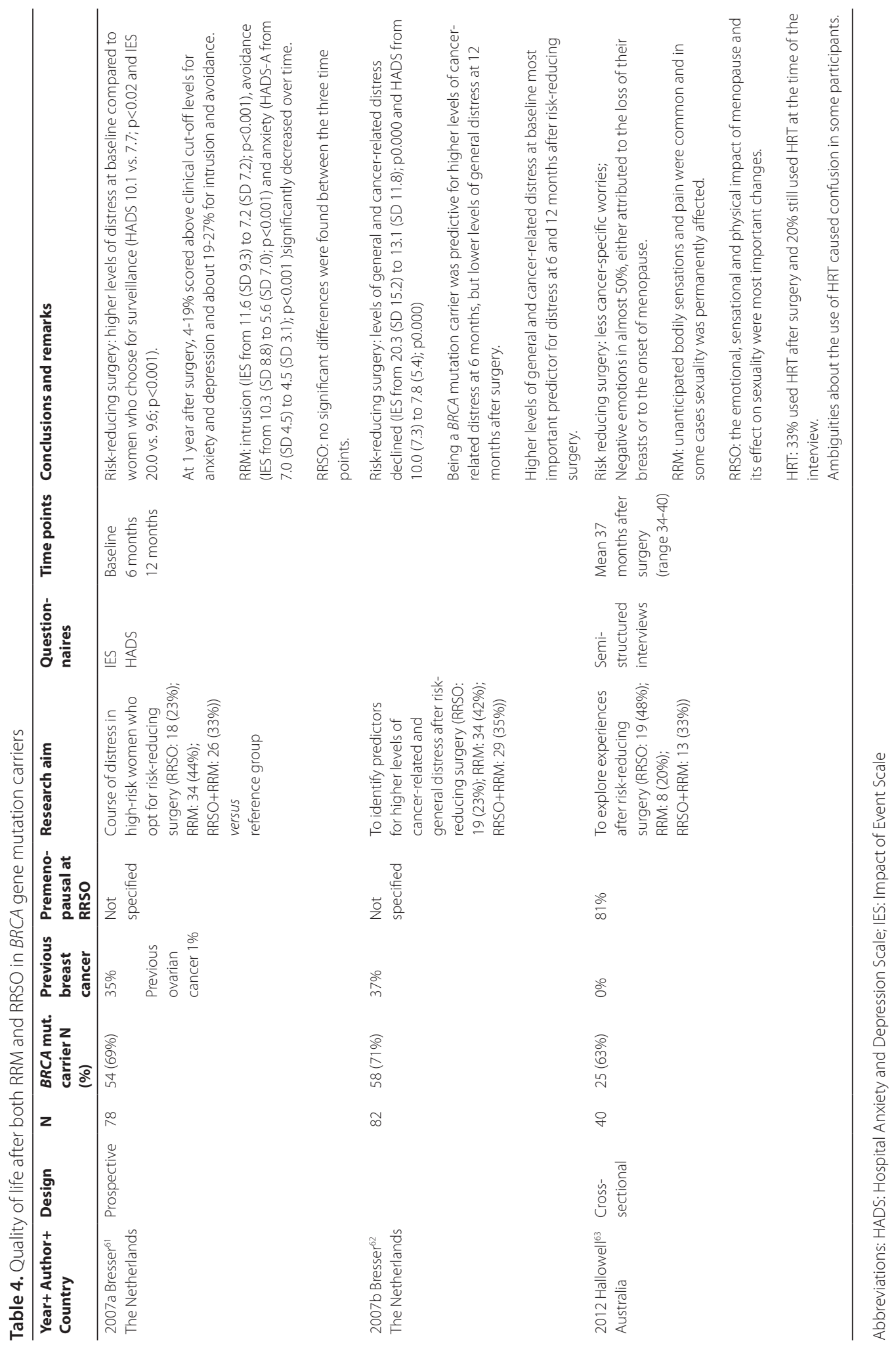




\section{DIScussion}

We reviewed the current literature about QoL in unaffected BRCA mutation carriers and how it is influenced by medical choices and interventions in cancer risk management. To the best of our knowledge, we are the first to report such a complete overview of different aspects of QoL in this particular population.

Most included studies are prospective cohort or cross-sectional studies with heterogeneity in study design, duration of follow-up and used questionnaires and with small sample sizes not limited to $B R C A$ mutation carriers alone. In a single study, results were not adjusted for differences between subgroups that already existed at baseline. Furthermore, due to the use of questionnaires and the duration of follow-up, most studies had to deal with selection bias, survival bias, the disadvantages of patient-reported data and loss of follow-up.

This review has some limitations. We assumed that women with a high familial risk of breast and ovarian cancer who were not or negatively tested for a germline BRCA mutation experience largely the same uncertainties and levels of anxiety as it comes to breast and ovarian cancer risk management as BRCA mutation carriers and therefore may have a comparable QoL. However, this was not always supported by subanalyses in the included studies: BRCA mutation carriers sometimes had higher distress levels, 32,34,35,37,45,62 lower body image, ${ }^{33}$ or greater increase of distress, ${ }^{41}$ whereas other studies stated that there were no differences in general QoL ${ }^{56,57}$ or even lower distress levels at some point. ${ }^{62}$ Moreover, studies were not always limited to unaffected women, especially in those on quality of life after RRSO. Some studies did not report subanalyses and we can imagine that women with a personal breast cancer history might score differently on health-related QoL items than unaffected women. ${ }^{28,64}$ Consequently, generalized results might not be applicable for the group of unaffected BRCA mutation carriers alone.

\section{Conclusion}

General QoL levels are comparable in unaffected BRCA mutation carriers and in the general population. Breast cancer surveillance visits, recalls after abnormal test results and RRM do not clearly affect overall QoL. No long-term effects on QoL were found after RRSO. Compared to women at high risk for breast and ovarian cancer without a BRCA mutation, BRCA mutation carriers had more cancer-specific distress, cancer worries, sleeping problems and higher cancer risk perception in the first year after genetic testing. Distress levels may fluctuate just before and after breast cancer surveillance visits. Recalls after abnormal MRI results temporarily increased levels of anxiety, avoidance and cancer worry. After risk-reducing surgery (both RRM and RRSO), levels of distress, anxiety and cancer worry decreased over time and women were highly satisfied with their decision. Levels of depression did not change. After RRSO, vasomotor symptoms and vaginal dryness were most frequently reported symptoms, bothering about a quarter to half of patients and being more persistent in women who were younger at the time of RRSO. BRCA mutation 
carriers had worse body image five years after genetic testing compared to non-carriers. After RRM, body image generally decreased. Sexual functioning after RRM was comparable to the level in the general population, but a slight decrease in the pleasure domain was observed. Sexual symptoms were more common after RRSO, especially those from the discomfort and pleasure domains, with women who were premenopausal at the time of RRSO most affected. HRT could partially alleviate endocrine and sexual symptoms after RRSO, but significant differences with women under surveillance remained.

In conclusion, general QoL appears not to be permanently affected in unaffected BRCA mutation carriers or by their choices in breast and ovarian cancer risk management. However, endocrine and sexual symptoms are very common after RRSO, especially in young women, and the beneficial effect of HRT is limited. Future research must concentrate on improving menopause-specific QoL in ovarian cancer risk management for BRCA mutation carriers, either by improving HRT or by postponing premature menopause in an alternative risk-reducing strategy. 


\section{REFEREMCES}

1. Russo A, Calo V, Bruno L, Rizzo S, Bazan V, Di Fede G. Hereditary ovarian cancer. Crit Rev Oncol Hematol 2009; 69(1): 28-44.

2. Chen S, Parmigiani G. Meta-analysis of BRCA1 and BRCA2 penetrance. J Clin Oncol 2007; 25(11): 1329-33.

3. Antoniou A, Pharoah PD, Narod $S$, et al. Average risks of breast and ovarian cancer associated with BRCA1 or BRCA2 mutations detected in case Series unselected for family history: a combined analysis of 22 studies. Am J Hum Genet 2003; 72(5): 1117-30.

4. Nelson HD, Fu R, Goddard K, et al. Risk Assessment, Genetic Counseling, and Genetic Testing for BRCARelated Cancer: Systematic Review to Update the US Preventive Services Task Force Recommendation. Rockville (MD); 2013.

5. Bordeleau L, Panchal S, Goodwin P. Prognosis of BRCA-associated breast cancer: a summary of evidence. Breast Cancer Res Treat 2010; 119(1): 13-24.

6. Vencken PM, Reitsma W, Kriege M, et al. Outcome of BRCA1- compared with BRCA2-associated ovarian cancer: a nationwide study in the Netherlands. Ann Oncol 2013; 24(8): 2036-42.

7. Hartmann LC, Sellers TA, Schaid DJ, et al. Efficacy of bilateral prophylactic mastectomy in BRCA1 and BRCA2 gene mutation carriers. J Natl Cancer Inst 2001; 93(21): 1633-7.

8. Meijers-Heijboer H, van Geel B, van Putten WL, et al. Breast cancer after prophylactic bilateral mastectomy in women with a BRCA1 or BRCA2 mutation. N Engl J Med 2001; 345(3): 159-64.

9. Heemskerk-Gerritsen BA, Menke-Pluijmers MB, Jager A, et al. Substantial breast cancer risk reduction and potential survival benefit after bilateral mastectomy when compared with surveillance in healthy BRCA1 and BRCA2 mutation carriers: a prospective analysis. Ann Oncol 2013; 24(8): 2029-35.

10. Rebbeck TR, Friebel T, Lynch HT, et al. Bilateral prophylactic mastectomy reduces breast cancer risk in BRCA1 and BRCA2 mutation carriers: the PROSE Study Group. J Clin Oncol 2004; 22(6): 1055-62.

11. Rebbeck TR, Kauff ND, Domchek SM. Meta-analysis of risk reduction estimates associated with riskreducing salpingo-oophorectomy in BRCA1 or BRCA2 mutation carriers. J Natl Cancer Inst 2009; 101(2): 80-7.

12. Marchetti C, De Felice F, Palaia I, et al. Risk-reducing salpingo-oophorectomy: a meta-analysis on impact on ovarian cancer risk and all cause mortality in BRCA 1 and BRCA 2 mutation carriers. BMC Womens Health 2014; 14(1): 150.

13. Domchek SM, Friebel TM, Singer CF, et al. Association of risk-reducing surgery in BRCA1 or BRCA2 mutation carriers with cancer risk and mortality. JAMA : the journal of the American Medical Association 2010; 304(9): 967-75.

14. Dowdy SC, Stefanek M, Hartmann LC. Surgical risk reduction: prophylactic salpingo-oophorectomy and prophylactic mastectomy. Am J Obstet Gynecol 2004; 191(4): 1113-23.

15. Atsma F, Bartelink ML, Grobbee DE, van der Schouw YT. Postmenopausal status and early menopause as independent risk factors for cardiovascular disease: a meta-analysis. Menopause 2006; 13(2): 265-79.

16. Rocca WA, Grossardt BR, Maraganore DM. The long-term effects of oophorectomy on cognitive and motor aging are age dependent. Neurodegener Dis 2008; 5(3-4): 257-60.

17. Shuster LT, Gostout BS, Grossardt BR, Rocca WA. Prophylactic oophorectomy in premenopausal women and long-term health. Menopause Int 2008; 14(3): 111-6.

18. Svejme O, Ahlborg HG, Nilsson JA, Karlsson MK. Early menopause and risk of osteoporosis, fracture and mortality: a 34-year prospective observational study in 390 women. BJOG 2012; 119(7): 810-6.

19. Arts-de Jong M, Maas AH, Massuger LF, Hoogerbrugge N, de Hullu JA. BRCA1/2 mutation carriers are potentially at higher cardiovascular risk. Crit Rev Oncol Hematol 2014.

20. Finch A, Metcalfe KA, Chiang JK, et al. The impact of prophylactic salpingo-oophorectomy on menopausal symptoms and sexual function in women who carry a BRCA mutation. Gynecol Oncol 2011; 121(1): 163-8. 
21. Metcalfe KA, Lubinski J, Ghadirian P, et al. Predictors of contralateral prophylactic mastectomy in women with a BRCA1 or BRCA2 mutation: the Hereditary Breast Cancer Clinical Study Group. J Clin Oncol 2008; 26(7): 1093-7.

22. Meiser B, Butow $\mathrm{P}$, Friedlander $\mathrm{M}$, et al. Intention to undergo prophylactic bilateral mastectomy in women at increased risk of developing hereditary breast cancer. J Clin Oncol 2000; 18(11): 2250-7.

23. Beattie MS, Crawford B, Lin F, Vittinghoff E, Ziegler J. Uptake, time course, and predictors of risk-reducing surgeries in BRCA carriers. Genet Test Mol Biomarkers 2009; 13(1): 51-6.

24. Singh K, Lester J, Karlan B, Bresee C, Geva T, Gordon O. Impact of family history on choosing risk-reducing surgery among BRCA mutation carriers. Am J Obstet Gynecol 2013; 208(4): 329 e1-6.

25. Stan DL, Shuster LT, Wick MJ, Swanson CL, Pruthi S, Bakkum-Gamez JN. Challenging and complex decisions in the management of the BRCA mutation carrier. J Womens Health (Larchmt) 2013; 22(10): 825-34.

26. Aaronson NK. Quality of life: what is it? How should it be measured? Oncology (Williston Park) 1988; 2(5): 69-76, 64.

27. Barofsky I. Can quality or quality-of-life be defined? Qual Life Res 2012; 21(4): 625-31.

28. Howard-Anderson J, Ganz PA, Bower JE, Stanton AL. Quality of life, fertility concerns, and behavioral health outcomes in younger breast cancer survivors: a systematic review. J Nat/ Cancer Inst 2012; 104(5): 386-405.

29. Montazeri A. Health-related quality of life in breast cancer patients: a bibliographic review of the literature from 1974 to 2007. J Exp Clin Cancer Res 2008; 27: 32.

30. van't Spijker A, ten Kroode HF. Psychological aspects of genetic counselling: a review of the experience with Huntington's disease. Patient Educ Couns 1997; 32(1-2): 33-40.

31. Lodder LN, Frets PG, Trijsburg RW, et al. One year follow-up of women opting for presymptomatic testing for BRCA1 and BRCA2: emotional impact of the test outcome and decisions on risk management (surveillance or prophylactic surgery). Breast Cancer Res Treat 2002; 73(2): 97-112.

32. Meiser B, Butow P, Friedlander M, et al. Psychological impact of genetic testing in women from high-risk breast cancer families. Eur J Cancer 2002; 38(15): 2025-31.

33. van Oostrom I, Meijers-Heijboer H, Lodder LN, et al. Long-term psychological impact of carrying a BRCA1/2 mutation and prophylactic surgery: a 5-year follow-up study. J Clin Oncol 2003; 21 (20): 386774.

34. Watson M, Foster C, Eeles R, et al. Psychosocial impact of breast/ovarian (BRCA1/2) cancer-predictive genetic testing in a UK multi-centre clinical cohort. Br J Cancer 2004; 91 (10): 1787-94.

35. Claes E, Evers-Kiebooms G, Denayer $L$, et al. Predictive genetic testing for hereditary breast and ovarian cancer: psychological distress and illness representations 1 year following disclosure. J Genet Couns 2005; 14(5): 349-63.

36. Foster C, Watson M, Eeles R, et al. Predictive genetic testing for BRCA1/2 in a UK clinical cohort: threeyear follow-up. Br J Cancer 2007; 96(5): 718-24.

37. Beran TM, Stanton AL, Kwan L, et al. The trajectory of psychological impact in BRCA1/2 genetic testing: does time heal? Ann Behav Med 2008; 36(2): 107-16.

38. Wenzel L, Osann K, Lester J, et al. Biopsychological stress factors in BRCA mutation carriers. Psychosomatics 2012; 53(6): 582-90.

39. Rijnsburger AJ, Essink-Bot ML, van Dooren S, et al. Impact of screening for breast cancer in high-risk women on health-related quality of life. Br J Cancer 2004; 91 (1): 69-76.

40. van Dooren S, Seynaeve C, Rijnsburger AJ, et al. Exploring the course of psychological distress around two successive control visits in women at hereditary risk of breast cancer. Eur J Cancer 2005; 41(10): 1416-25.

41. O'Neill SM, Rubinstein WS, Sener SF, et al. Psychological impact of recall in high-risk breast MRI screening. Breast Cancer Res Treat 2009; 115(2): 365-71. 
42. Spiegel TN, Esplen MJ, Hill KA, Wong J, Causer PA, Warner E. Psychological impact of recall on women with BRCA mutations undergoing MRI surveillance. Breast 2011; 20(5): 424-30.

43. den Heijer M, Seynaeve C, Vanheusden K, et al. Long-term psychological distress in women at risk for hereditary breast cancer adhering to regular surveillance: a risk profile. Psychooncology 2013; 22(3): 598604.

44. Brandberg Y, Arver B, Lindblom A, Sandelin K, Wickman M, Hall P. Preoperative psychological reactions and quality of life among women with an increased risk of breast cancer who are considering a prophylactic mastectomy. Eur J Cancer 2004; 40(3): 365-74.

45. Metcalfe KA, Esplen MJ, Goel V, Narod SA. Psychosocial functioning in women who have undergone bilateral prophylactic mastectomy. Psychooncology 2004; 13(1): 14-25.

46. Metcalfe KA, Esplen MJ, Goel V, Narod SA. Predictors of quality of life in women with a bilateral prophylactic mastectomy. Breast J 2005; 11 (1): 65-9.

47. Brandberg Y, Sandelin K, Erikson S, et al. Psychological reactions, quality of life, and body image after bilateral prophylactic mastectomy in women at high risk for breast cancer: a prospective 1-year followup study. J Clin Oncol 2008; 26(24): 3943-9.

48. Isern AE, Tengrup I, Loman N, Olsson H, Ringberg A. Aesthetic outcome, patient satisfaction, and healthrelated quality of life in women at high risk undergoing prophylactic mastectomy and immediate breast reconstruction. J Plast Reconstr Aesthet Surg 2008; 61(10): 1177-87.

49. den Heijer M, Seynaeve C, Timman R, et al. Body image and psychological distress after prophylactic mastectomy and breast reconstruction in genetically predisposed women: a prospective long-term follow-up study. Eur J Cancer 2012; 48(9): 1263-8.

50. Gopie JP, Mureau MA, Seynaeve C, et al. Body image issues after bilateral prophylactic mastectomy with breast reconstruction in healthy women at risk for hereditary breast cancer. Fam Cancer 2013; 12(3): 479-87.

51. Draycott S, Dabbs A. Cognitive dissonance. 1: An overview of the literature and its integration into theory and practice in clinical psychology. Br J Clin Psychol 1998; 37 ( Pt 3): 341-53.

52. Fang CY, Cherry C, Devarajan K, Li T, Malick J, Daly MB. A prospective study of quality of life among women undergoing risk-reducing salpingo-oophorectomy versus gynecologic screening for ovarian cancer. Gynecol Oncol 2009; 112(3): 594-600.

53. Finch A, Metcalfe KA, Chiang J, et al. The impact of prophylactic salpingo-oophorectomy on quality of life and psychological distress in women with a BRCA mutation. Psychooncology 2013; 22 (1): 212-9.

54. Madalinska JB, Hollenstein J, Bleiker E, et al. Quality-of-life effects of prophylactic salpingo-oophorectomy versus gynecologic screening among women at increased risk of hereditary ovarian cancer. J Clin Oncol 2005; 23(28): 6890-8.

55. Michelsen TM, Dorum A, Dahl AA. A controlled study of mental distress and somatic complaints after risk-reducing salpingo-oophorectomy in women at risk for hereditary breast ovarian cancer. Gynecol Oncol 2009; 113(1): 128-33.

56. Michelsen TM, Dorum A, Trope CG, Fossa SD, Dahl AA. Fatigue and quality of life after risk-reducing salpingo-oophorectomy in women at increased risk for hereditary breast-ovarian cancer. Int J Gynecol Cancer 2009; 19(6): 1029-36.

57. Touboul C, Uzan C, Ichante $\mathrm{J}$, et al. Factors associated with altered long-term well-being after prophylactic salpingo-oophorectomy among women at increased hereditary risk for breast and ovarian cancer. Oncologist 2011; 16(9): 1250-7.

58. Pezaro C, James P, McKinley J, Shanahan M, Young MA, Mitchell G. The consequences of risk reducing salpingo-oophorectomy: the case for a coordinated approach to long-term follow up post surgical menopause. Fam Cancer 2012; 11(3): 403-10.

59. Madalinska JB, van Beurden M, Bleiker EM, et al. The impact of hormone replacement therapy on menopausal symptoms in younger high-risk women after prophylactic salpingo-oophorectomy. J Clin Oncol 2006; 24(22): 3576-82. 
60. Chapman JS, Powell CB, McLennan J, et al. Surveillance of survivors: follow-up after risk-reducing salpingo-oophorectomy in BRCA 1/2 mutation carriers. Gynecol Oncol 2011; 122(2): 339-43.

61. Bresser PJ, Seynaeve C, Van Gool AR, et al. The course of distress in women at increased risk of breast and ovarian cancer due to an (identified) genetic susceptibility who opt for prophylactic mastectomy and/ or salpingo-oophorectomy. Eur J Cancer 2007; 43(1): 95-103.

62. Bresser PJ, Van Gool AR, Seynaeve C, et al. Who is prone to high levels of distress after prophylactic mastectomy and/or salpingo-ovariectomy? Ann Oncol 2007; 18(10): 1641-5.

63. Hallowell N, Baylock B, Heiniger $L$, et al. Looking different, feeling different: women's reactions to riskreducing breast and ovarian surgery. Fam Cancer 2012; 11(2): 215-24.

64. Mols F, Vingerhoets AJ, Coebergh JW, van de Poll-Franse LV. Quality of life among long-term breast cancer survivors: a systematic review. Eur J Cancer 2005; 41(17): 2613-9. 

inc.

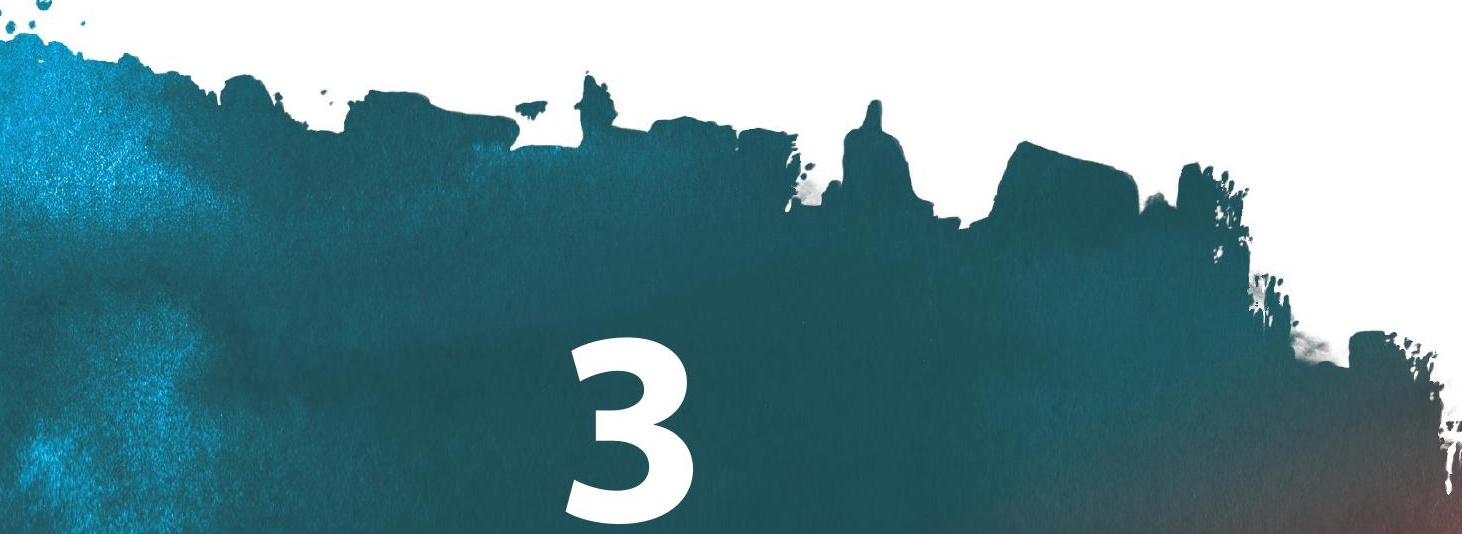

Veny high uptake of risk-reducing salpingo-oophorectomy in BRCA1/2 mutation camriens:

a single-center experience

Marline G. Harmsen, Marieke Arts-de Jong, Kim Horstik, Peggy Manders, Leon F.A.G. Massuger, Rosella P.M.G. Hermens, Nicoline Hoogerbrugge, Gwendolyn H. Woldringh, Joanne A. de Hullu 


\section{ABSTRACT}

\section{Objective}

Risk-reducing salpingo-oophorectomy (RRSO) is the only effective surgical strategy to reduce the increased risk of epithelial ovarian cancer in BRCA1/2 mutation carriers. Given the long-term health consequences of premature surgical menopause, we need insight in uptake and timing of RRSO to guide us in improving healthcare.

\section{Methods}

A single-center retrospective cohort study of BRCA1/2 mutation carriers diagnosed and counseled at the multidisciplinary Family Cancer Clinic of the Radboud university medical center in Nijmegen, The Netherlands between 1999 and 2014. Descriptive statistics were used to analyze uptake and timing of RRSO.

\section{Results}

Data of $580 B R C A 1 / 2$ were analyzed. The uptake of RRSO among mutation carriers who are currently above the upper limit of the recommended age for RRSO, is $98.5 \%$ and $97.5 \%$ for BRCA1 and BRCA2 mutation carriers, respectively. The vast majority undergoes RRSO $\leq 40$ (BRCA1) or $\leq 45$ (BRCA2) years of age, provided that mutation status is known by that age: $90.8 \%$ and $97.3 \%$ of BRCA1 and BRCA2 mutation carriers, respectively.

\section{Conclusions}

The uptake of RRSO among BRCA1/2 mutation carriers who were counseled at our Family Cancer Clinic is extremely high. High uptake might be largely attributed to the directive and uniform way of counseling by professionals at our Family Cancer Clinic. Given the fact that RRSO is often undergone at premenopausal age in our population, future research should focus on minimizing long-term health consequences of premature surgical menopause either by optimization of hormone replacement therapy or by investigating alternative strategies to RRSO. 


\section{InTRODUCTION}

The cumulative risk of developing epithelial ovarian cancer (EOC) at age 70 in women harboring a germline BRCA1 or BRCA2 mutation is about 39\% (95\% confidence interval 34-44\%) and 16\% (95\% confidence interval 12-20\%), respectively. ${ }^{1}$ Most women with EOC present with advanced-stage disease leading to a poor five-year survival. Screening for ovarian cancer using ultrasound and serum levels of tumor marker Ca125 has been proven ineffective in BRCA1/2 mutation carriers. ${ }^{2}$ Risk-reducing salpingo-oophorectomy (RRSO) is the only effective surgical intervention in the prevention of EOC, reducing the risk by at least $80 \%{ }^{3,4}$

EOC risk increases at the end of the fourth decade in BRCA1 mutation carriers, and from the beginning of the sixth decade in BRCA2 mutation carriers. ${ }^{5}$ Most international guidelines recommend RRSO to both BRCA1 and BRCA2 mutation carriers between age 35 and 40 if childbearing is completed. . $^{6,7}$ The Dutch guideline, however, slightly differs from these international guidelines. Initially, it was recommended to undergo RRSO before the age of 40 or five years prior to the youngest age at which a relative was diagnosed with EOC to all BRCA mutation carriers. Since 2010, distinction is made in recommended age for RRSO between BRCA1 and BRCA2 in Dutch daily practice: between 35 and 40 years for $B R C A 1$ and between 40 and 45 years for $B R C A 2 .{ }^{8}$ This distinction is based on the risk of EOC increasing at older age in BRCA2 mutation carriers.

Uptake of RRSO varies from $17 \%$ to $89 \%$ worldwide. ${ }^{9}$ Research on uptake of risk-reducing surgery in BRCA1/2 mutation carriers has mainly been concentrated on demographic and psychosocial predictors. ${ }^{10,11}$ Main predictors for uptake of RRSO are family history of EOC, personal history of breast cancer, and age older than 40 years. ${ }^{12}$ However, a turning point with respect to age has been observed as well, i.e. women older than 60 years were less inclined to undergo RRSO.13,14

The objective of this study was to investigate the uptake of RRSO among BRCA1/2 mutation carriers in our tertiary hospital with a Family Cancer Clinic, and whether RRSO is usually performed within the recommended age range.

\section{MATERIALS AMD METHODS}

\section{Study population}

A retrospective single-center study was performed among all women diagnosed with a germline $B R C A 1 / 2$ mutation at the laboratory of Human Genetics of the Radboud university medical center in Nijmegen, The Netherlands, between January 1999 and December 2012. Only BRCA1/2 mutation carriers who were subsequently counseled at the Family Cancer Clinic of the Radboud university medical center were included. The integrated care for BRCA1/2 mutation carriers at the Family Cancer Clinic is organized within the context of a multidisciplinary team including all professionals involved in prevention and management of either breast or ovarian cancer: clinical geneticists, 
gynecologic oncologists, medical oncologists, (plastic) surgeons, pathologists, radiologists, and social workers. Mutation carriers and their families are discussed within this multidisciplinary team during a weekly session in order to coordinate care and to avoid mixed messages to these patients. According to the Dutch law, no Institutional Board Review approval was needed for this retrospective study of medical records in which participants' anonymity was guaranteed by assigning studyspecific, unique patient numbers.

\section{Setting}

For the 17 million inhabitants of The Netherlands, genetic counseling and germline BRCA1/2 mutation testing is concentrated in eight university medical centers and one specialized cancer institute. The laboratory that identifies the first BRCA1/2 mutation within a family will test all following family members, independent of the center in which counseling takes place. After diagnosis of a germline BRCA1/2 mutation, women are generally referred for counseling on breast and ovarian cancer risk management by a specialist in breast surveillance and a gynecologic oncologist at the Family Cancer Clinic of one of the nine above-mentioned centers.

During counseling at the Radboud university medical center, BRCA1 and BRCA2 mutation carriers are told that estimated lifetime risks of EOC are 30-60\% and 5-20\%, respectively. Furthermore, it was emphasized that RRSO is the only evidence-based approach to reduce this elevated risk of EOC, and recommended ages for RRSO are mentioned. Until 2010, recommended ages for RRSO were equal for both BRCA1 and BRCA2 mutation carriers: between 35 and 40 years old, or five years before the age of onset of $\mathrm{EOC}$ in a relative if that relative was diagnosed with $\mathrm{EOC}$ before the recommended age of RRSO. In 2010, however, the recommended age for RRSO for BRCA2 mutation carriers was increased to 40-45 years. Use of hormone replacement therapy is recommended from RRSO until the age of 48-50, if not contra-indicated because of a breast cancer history. Additionally, breast cancer risk reduction by half when RRSO was performed before onset of natural menopause was communicated to all women in this cohort, based on the available evidence at that time. Lastly, $B R C A 1 / 2$ mutation carriers in our hospital were offered annual ovarian cancer screening consisting of transvaginal ultrasounds and Ca125 testing until September 2011. Since then, patients have been counseled about the ineffectiveness of ovarian cancer screening in terms of prognosis and false positive findings, and ovarian screening have not routinely been offered anymore. An overview of key elements addressed in each counseling session by a gynecologic oncologist at our Family Cancer Clinic can be found in Box 1. 
Box 1. Key points emphasized by gynecologic oncologist during counseling sessions

- Cumulative risk of EOC 30-60\% (BRCA1) and 5-20\% (BRCA2)

- Poor survival of EOC

- Ovarian screening is ineffective (since September 2011)

- $\quad$ RRSO is the only evidence-based approach to reduce EOC risk

- Recommended age for RRSO: age 35-40 (and age 40-45 for BRCA2 since 2010)

- $\quad$ Breast cancer risk reduced by half if performed before natural menopause (until 2015)

- No need for prophylactic hysterectomy

- Use of hormone replacement therapy is recommended until the age of 48-50 if not contraindicated (e.g. breast cancer history)

\section{Data collection}

Information on demographics, clinical characteristics and risk-reducing surgeries was obtained from medical records, including age at BRCA1/2 mutation diagnosis, mutation status, parity, family history of breast and ovarian cancer, personal history of breast and ovarian cancer, date of RRSO, and time from BRCA diagnosis to RRSO (time to surgery). Consultation of medical records took place in April 2014. Whenever medical records did not mention RRSO, the nationwide network and registry of histo- and cytopathology in the Netherlands (PALGA) was consulted to find out whether and when RRSO was possibly undergone in another Dutch hospital.

\section{Analyses}

All statistical analyses were performed using IBM SPSS Statistics version 22. Descriptive statistics were used to summarize patient characteristics and to present uptake and age at RRSO. RRSO within the recommended age is up to and including age 40 for BRCA1, and up to and including age 45 for BRCA2 mutation carriers. We determined the median time from diagnosis of the BRCA1/2 mutation to RRSO in three groups classified by age at mutation diagnosis: before, at or after the recommended age for RRSO. Differences in time to surgery between women with and without a personal history of breast cancer at the time of mutation diagnosis were tested for statistical significance using the Mann-Whitney test for non-parametrically distributed data.

\section{RESULTS}

\section{Population}

A germline BRCA1/2 mutation was diagnosed in 1,038 women between January 1999 and December 2012. Of these, 609 visited the Family Cancer Clinic at least once with first visits between April 1999 and February 2014. Twelve women were excluded because they had already undergone bilateral salpingo-oophorectomy: eight as risk-reductive strategy because of their family history before they knew their mutation status and four for other non-oncological reasons. Sixteen women had already developed EOC before the BRCA1/2 mutation was diagnosed. One BRCA1 mutation carrier 
was excluded because she died of metastatic breast cancer before she reached the recommended age for RRSO. The remaining 580 women were included in our analyses: 357 BRCA1, 222 BRCA2, and $1 B R C A 1+2$ mutation carriers. The woman who carried germline mutations in both BRCA1 and $B R C A 2$ was counseled and analyzed as a BRCA1 mutation carrier. Patient characteristics of all 580 included women are shown in Table 1. A flowchart can be found in Figure 1.

A total number of 209 BRCA1 mutation carriers were diagnosed with the mutation at $\leq 40$ years (58.4\%), and 149 women were diagnosed at a higher age (41.6\%). A BRCA2 mutation was diagnosed at $\leq 45$ years in 139 women (62.6\%), and at higher age in 83 women (37.4\%).

After BRCA1/2 mutation diagnosis, two women developed pelvic cancer: one 52-year-old BRCA1 mutation carrier had primary peritoneal carcinoma four years after RRSO, and one 60-year-old BRCA2 mutation carrier was diagnosed with EOC at the time of RRS.

Table 1. Characteristics of study population

\begin{tabular}{|c|c|c|c|c|}
\hline & $B R C A$ & $1+2)$ & & \\
\hline & $N=358$ & $61.8 \%$ & $\mathrm{~N}=222$ & $38.2 \%$ \\
\hline & Median & Range & Median & Range \\
\hline Age at mutation diagnosis (yr) & 37.5 & $18-79$ & 41.0 & 19-79 \\
\hline & $N$ & $\%$ & $N$ & $\%$ \\
\hline Parity & & & & \\
\hline 0 & 82 & $22.9 \%$ & 46 & $20.7 \%$ \\
\hline $1-2$ & 200 & $55.9 \%$ & 114 & $51.3 \%$ \\
\hline$\geq 3$ & 72 & $20.1 \%$ & 60 & $27.1 \%$ \\
\hline Missing & 4 & $1.1 \%$ & 2 & $0.9 \%$ \\
\hline Menopausal status at $1^{\text {st }}$ gynecold & & & & \\
\hline Premenopausal & 256 & $71.5 \%$ & 148 & $66.7 \%$ \\
\hline Postmenopausal & 93 & $26.0 \%$ & 69 & $31.1 \%$ \\
\hline Missing & 9 & $2.5 \%$ & 5 & $2.2 \%$ \\
\hline Personal history of breast cancer & 84 & $23.5 \%$ & 51 & $23.0 \%$ \\
\hline At mutation diagnosis & 54 & $15.1 \%$ & 39 & $17.6 \%$ \\
\hline Missing & 2 & $0.6 \%$ & 0 & - \\
\hline First degree family history & & & & \\
\hline Breast cancer & 195 & $54.5 \%$ & 111 & $50 \%$ \\
\hline Missing & 41 & $11.5 \%$ & 33 & $14.9 \%$ \\
\hline Ovarian cancer & 88 & $24.6 \%$ & 19 & $8.6 \%$ \\
\hline Missing & 37 & $10.3 \%$ & 27 & $12.2 \%$ \\
\hline
\end{tabular}




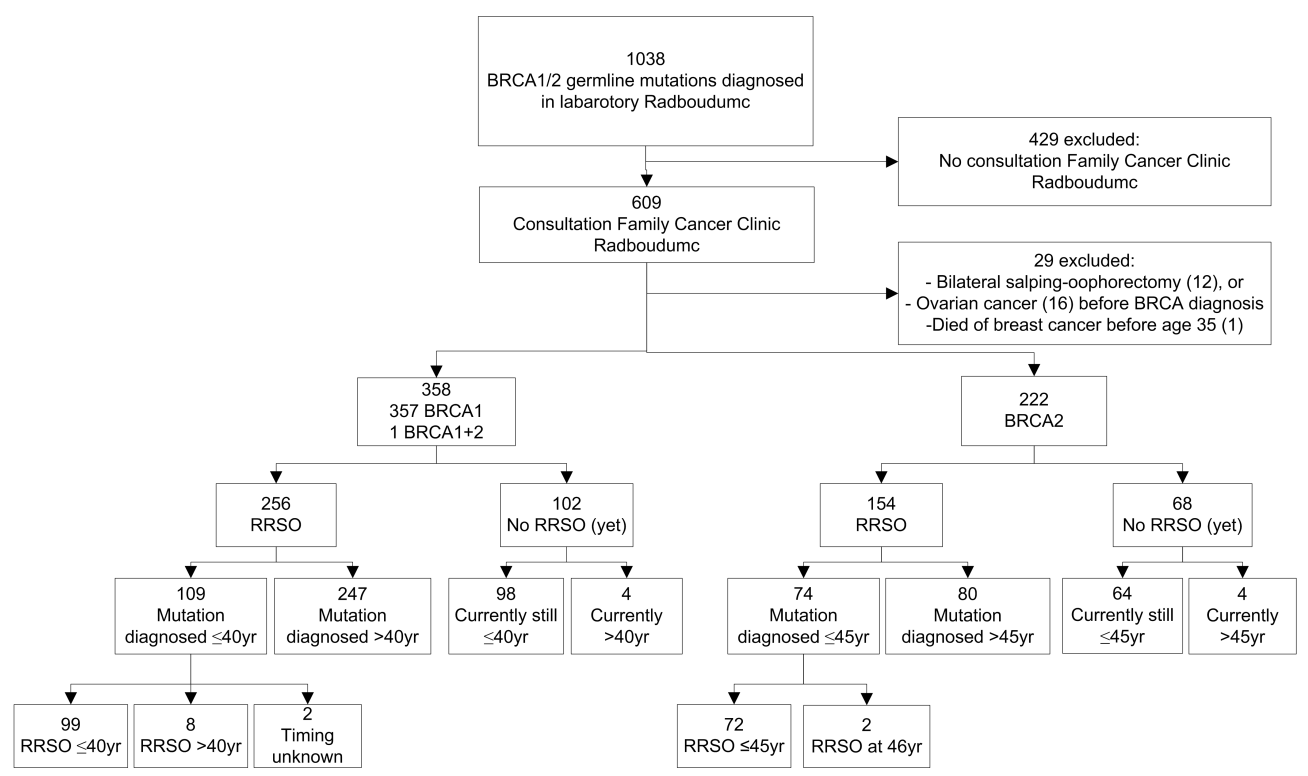

Figure 1. Flowchart

\section{Uptake and timing of RRSO}

Of all 358 BRCA1 mutation carriers 98 are still $\leq 40$ years and have not yet undergone RRSO, probably because of age. Of the remaining 260 women, four have not undergone RRSO yet despite their current age of 41 years or older. Total uptake is $98.5 \%$.

A total number of 209 BRCA1 mutation carriers had their mutation diagnosed at age 40 or younger. Of them, 98 are still $\leq 40$ years old and have not yet undergone RRSO, as described above. The other 111 are currently 41 years or older of which 109 have already undergone RRSO: 99 before or at 40 years of age. Eight women underwent RRSO at higher age: six were already 40 years old at the time of genetic test disclosure and underwent RRSO within one year from then, and two of them had other reasons to postpone RRSO (Table 2). Timing of RRSO of two BRCA1 mutation carriers is unknown.

Sixty-four of a total of 222 BRCA2 mutation carriers are currently still 45 years old or younger and have not yet undergone RRSO, probably because of age. Of the remaining 158, four women have not undergone RRSO yet despite the fact that they are currently 46 years or older (Table 2). Total uptake of RRSO among BRCA2 mutation carriers is $97.5 \%$. Of the 75 BRCA2 mutation carriers who are currently 46 years or older and had their mutation diagnosed at age 45 or younger, 74 underwent RRSO, of which 72 before or at the age of 45 . Two women underwent RRSO at age 46 within one year after detection of their germline mutation at age 45 .

Only two of 149 BRCA1 mutation carriers who were older than 40 years at the time of mutation detection decided not to undergo RRSO. Of all 83 women who had their BRCA2 mutation diagnosed after the age of 45 , three have not undergone RRSO yet. Reasons for reluctance can be found in Table 2. 


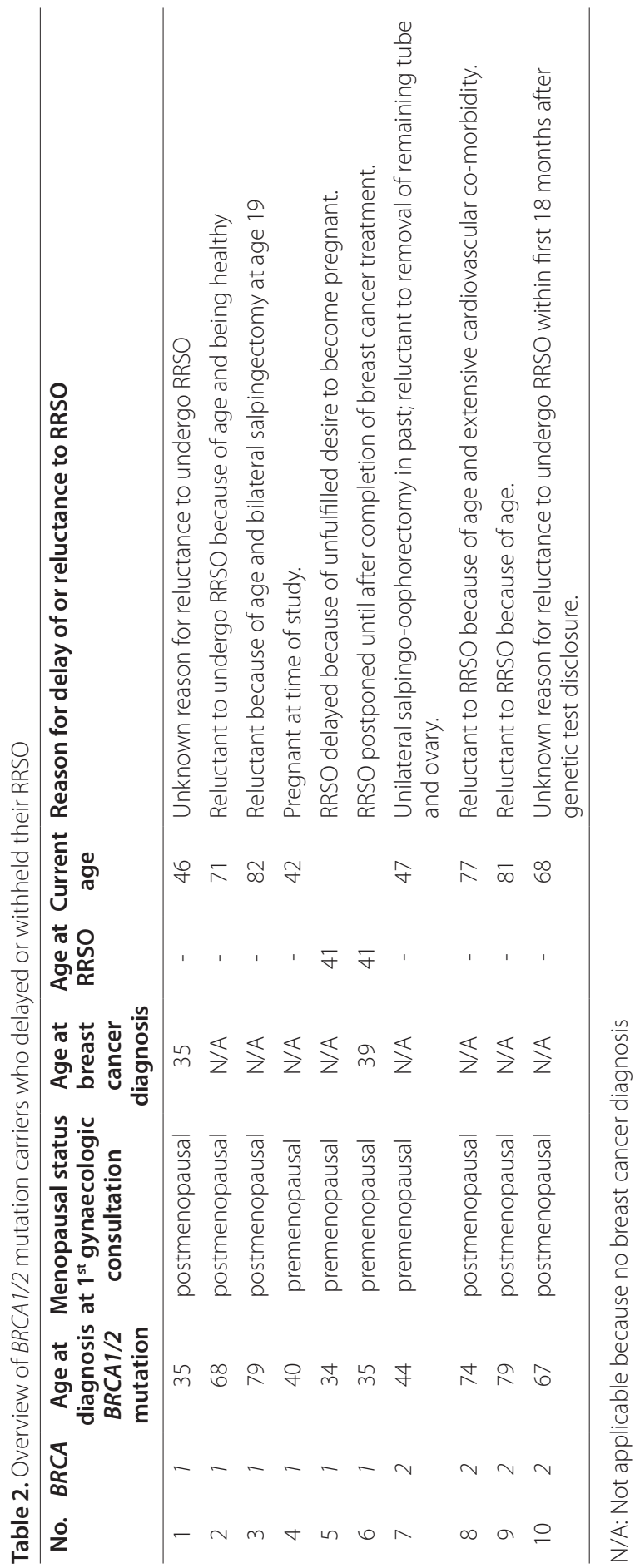




\section{BRCA2 mutation carriers before and after changing recommended age for RRSO}

Hundred thirty-five BRCA2 mutation carriers visited the Family Cancer Clinic for the first time before January 2010 and 87 in 2010 or thereafter. Sixty-eight of 135 women were $\leq 40$ years old at this first visit and were recommended to undergo RRSO $\leq 40$ years of age, of which 34 (50\%) have not undergone RRSO yet. They all are currently $\leq 44$ years old, so they were $\leq 40$ years old in 2010 when the recommended age was changed and they were acquainted about this new recommendation. Of the 34 BRCA2 mutation carriers who have already undergone RRSO (median age at RRSO 40 years, range 32-44), 27 were 40 years old or younger at the time of surgery and seven were 41 years or older and thus crossed the upper limit of the previously recommended age. However, three of them had not reached the age of 40 by 2010 and adhered to the currently recommended age to undergo RRSO: they underwent RRSO at age 41. Two others were 40 years old at the time of mutation detection and subsequently underwent RRSO at age 41 as well. The only two women (5.9\%) who really deviated from the previous guideline both underwent RRSO at age 44 in 2010. One of them initially preferred annual ovarian surveillance to RRSO, and the reason for the other woman is unknown because she did not have follow-up in our hospital.

Of 87 BRCA2 mutation carriers who were recommended to undergo RRSO between 40 and 45 years of age because they primarily visited the Family Cancer Clinic from January 2010 onwards, 34 had their mutation diagnosed before or at 40 years of age. Thirty-one of them have not undergone RRSO yet because of age: only one is currently 42 years old and 30 are currently $\leq 37$ years old. The other three did undergo RRSO: two at age 40 and one at age 39. Only the latter deviated from the recommended age by undergoing surgery 2.5 months before her $40^{\text {th }}$ birthday.

The number of BRCA2 mutation carriers who were diagnosed $\leq 40$ years and visited our Family Cancer Clinic for the first time between January 2010 and September 2013 is too small to draw conclusions about implementation of this new recommendation, taken into account that 30 of 34 patients have not reached the age of 40 yet.

\section{Time to surgery}

Time to surgery related to breast cancer status at the time of mutation diagnosis could be determined for 405 of 410 BRCA1/2 mutation carriers who underwent RRSO. Dates of RRSO were missing for two BRCA1 mutation carriers, and data on breast cancer status at mutation diagnosis were missing for three other BRCA1 mutation carriers. Box plots for these 405 women are presented in Figure 2 (BRCA1) and Figure 3 (BRCA2).

For BRCA1 mutation carriers, median time to surgery was only statistically significantly longer in women who were affected with breast cancer (median 5 months) compared to unaffected women (median 4 months) in the group of women 40 years and older at the time of mutation diagnosis (Mann-Whitney $U=2,912.00, z=2.602, p=0.009$ ). However, the difference of only one month does not seem clinically relevant. Among BRCA1 mutation carriers regardless of age at mutation diagnosis, median time to surgery was 5 months in unaffected and 6 months in affected women ( $p=0.807)$. 


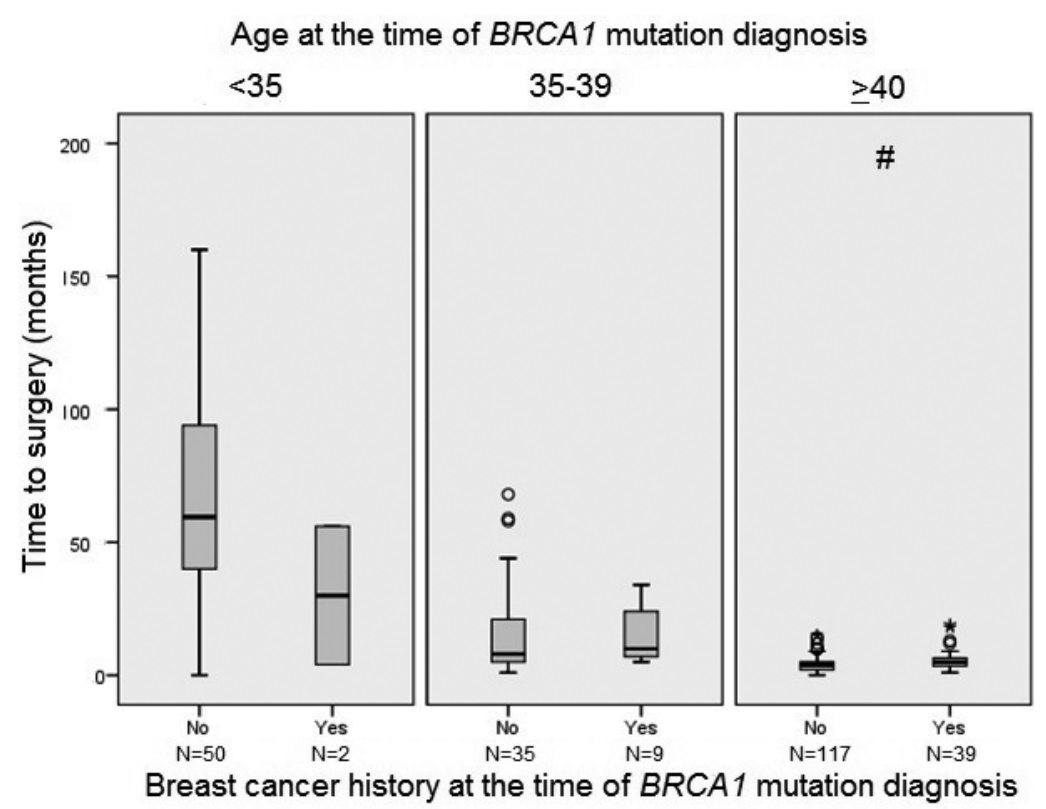

Figure 2. Time to surgery in BRCA1 mutation carriers given the age at mutation diagnosis and personal breast cancer history. \#: statistically significant (Mann-Whitney test)

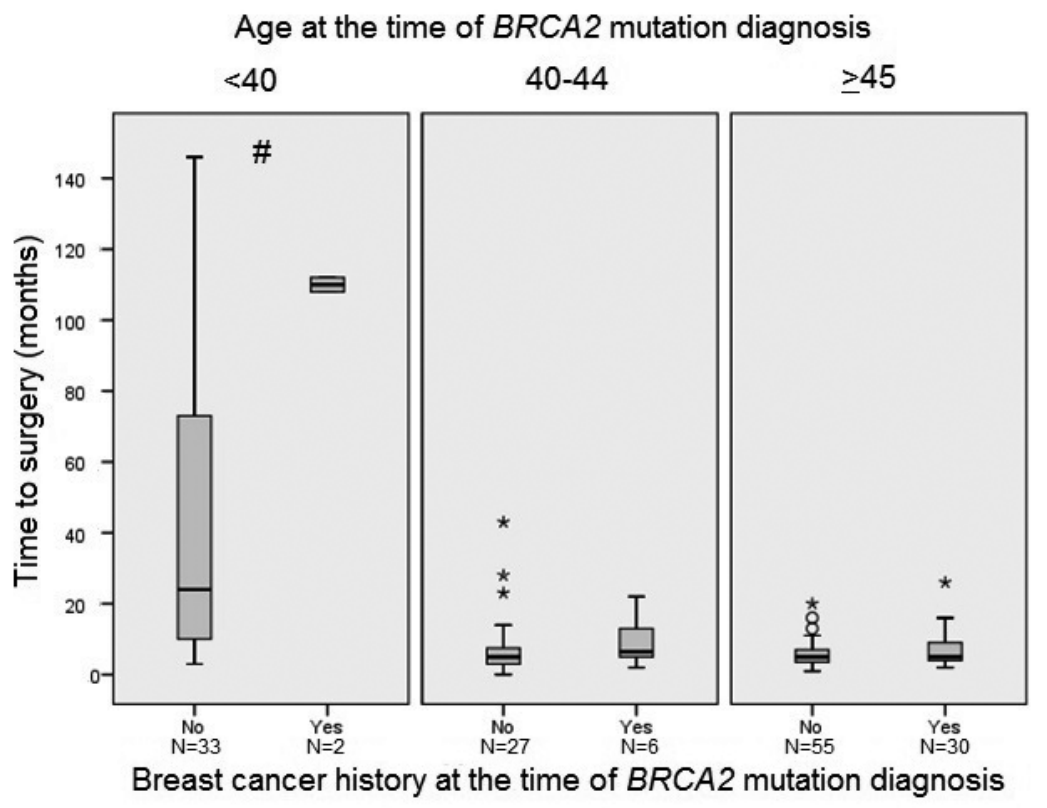

Figure 3. Time to surgery in BRCA2 mutation carriers given the age at mutation diagnosis and personal breast cancer history. \#: statistically significant (Mann-Whitney test) 
For BRCA2 mutation carriers, the only statistically significant difference was found within women who were diagnosed with the mutation before the recommended age for RRSO ( $<40$ years). Time to surgery was longer in affected (median 110 months) compared to unaffected women (median 24 months) (Mann-Whitney $U=63.00, z=2.134, p=0.020$ ). However, these results need to be interpreted with caution because only two women with a breast cancer history at the time of mutation diagnosis were included in this age group. Regardless of age at mutation diagnosis, median time to RRSO was 6 months both for unaffected and affected BRCA2 mutation carriers $(p=0.532)$.

\section{DIScussion}

Among BRCA1/2 mutation carriers who visited our Family Cancer Clinic, uptake of RRSO is very high with $98.5 \%$ and $97.5 \%$ among BRCA1 and BRCA2 mutation carriers, respectively. Furthermore, the vast majority undergoes RRSO at the recommended age, provided, that the germline BRCA1/2 mutation is diagnosed before the upper limit of the recommended age. Time to RRSO does not convincingly differ between women with and without a personal breast cancer history at the time of mutation diagnosis.

The best explanation for the extremely high uptake is probably the very directive way of counseling by all professionals working at the Family Cancer Clinic. Besides, no alternative in terms of ovarian cancer screening has been offered since September 2011; however, even before 2011, the option of annual screening seems to have contributed to postponement of only one RRSO beyond the age that was recommended at that time. For three others, reasons for postponing or refusing RRSO are unknown.

The reported uptake of RRSO among women at high risk of breast and ovarian cancer varies widely across the world: from 17 to 52.4\% in Asia, ${ }^{9,15}$ from 17.3 to 38\% in Australia, ${ }^{9}$ from 40-89.5\% in North America, ${ }^{9,16,17}$ and from 21.4 to $75 \%$ across European countries outside the Netherlands., ${ }^{9,18}$ From other parts of the Netherlands, uptake rates of 64 to $87.2 \%$ have been reported. ${ }^{19-22}$ Several aspects can contribute to the differences in reported uptake rates.

Firstly, methodological differences are present across studies: uptake of RRSO can widely vary depending on the study population (e.g. older age, personal breast cancer history and BRCA1/2 carrier status have been associated with higher uptake). ${ }^{12}$ We only included proven BRCA1/2 mutation carriers and we included both carriers with and without a personal history of breast cancer. Furthermore, for women who have not yet undergone RRSO in our study, we only counted those who have already passed the recommended age for RRSO. In our opinion, it is only relevant to measure uptake in women above the recommended age because EOC risk slightly starts to increase at that age and thus they are at an actual risk. Also, it is very likely that women at or below the recommended age will still undergo RRSO within the recommended age range, given our 
study data. Considering them reluctant to RRSO too early would be misleading. However, excluding women who have not yet exceeded the currently recommended age (i.e. $\leq 40$ years for BRCA1 and $\leq 45$ years for BRCA2) might partially explain the high uptake in our population compared to other studies. In addition, uptake can also depend on determination of a certain time frame, e.g. uptake within the first 12 months after genetic test disclosure. We did not determine such a time frame. More than five years of follow-up in the majority of women in this study could contribute to our higher RRSO uptake, since women still elected RRSO several years after genetic test disclosure.

Secondly, physicians' attitudes towards risk-reducing surgery diverge internationally, which may influence counseling of BRCA mutation carriers and thereby their uptake of RRSO.23-29 Counseling can be more or less directive: Laitman et al. ${ }^{30}$ found 51\% uptake in an Israeli population in case the healthcare provider recommended RRSO versus 39\% uptake in case the healthcare provider advised against RRSO. The authors suggested that counseling by physicians who were not members of the oncogenetics team may be a reason for low RRSO uptake. In addition to this, counseling by a gynecologic oncologist has been described to increase uptake: Kim et al. ${ }^{15}$ observed an uptake of 21/33 (66\%) among Korean BRCA1/2 mutation carriers who were counseled by a gynecologic oncologist in contrast to an uptake of only $1 / 9$ (11\%) among those who were counseled by other professionals (not further specified). At last, recommending the use of hormone replacement therapy after RRSO until the age of 48-50 may increase its acceptance and uptake. ${ }^{31}$ Therefore, very unequivocal recommendations provided by gynecologic oncologists at our multidisciplinary Family Cancer Clinic may have contributed to the very high RRSO uptake in our study.

Thirdly, RRSO uptake can be affected by offering alternative options. Specifically, the possibility of less invasive ovarian cancer screening might reduce the uptake of RRSO, although proven ineffective. This is supported by a Dutch study: ${ }^{19}$ overall uptake of RRSO within one year after genetic test disclosure increased from 53\% to 85\% after stopping ovarian cancer screening, and from 77\% to $91 \%$ among women who reached the recommended age for RRSO. Offering screening as part of a clinical trial was mentioned as a possible explanation for the low uptake of RRSO observed in women at risk of familial EOC in the United Kingdom (both BRCA mutation carriers and women with unknown BRCA status). ${ }^{18}$

Furthermore, taking into account the formerly assumed effect of premenopausal RRSO on breast cancer risk reduction might have increased uptake of RRSO: ${ }^{17}$ from our experience, BRCA1/2 mutation carriers, especially in families with a high breast cancer incidence, preferred halving their breast cancer risk through RRSO over minimizing that risk by risk-reducing mastectomy, the latter being more effective but (experienced as) more invasive and mutilating on the other hand. However, a recent Dutch study found no beneficial effect of RRSO on breast cancer incidence after applying alternative analyses to earlier large cohort studies, thereby minimizing amounts of bias. ${ }^{32}$ Therefore, breast cancer risk has not been part of counseling on RRSO anymore in our center since 2015, of which the effect on RRSO uptake is unknown up to now. 
Lastly, differences in uptake of RRSO between continents might also be attributed to cultural differences, e.g. women's devotion to retain fertility as long as possible, their attitude towards menopause, whether RRSO is considered as mutilation and whether paternalism is more common in healthcare instead of emphasis on patient's autonomy. 25,26,33 In the Netherlands specifically, several factors can also contribute to a high uptake of RRSO as was described for Denmark: free access to healthcare, costs of risk-reducing surgery covered by compulsory health insurance, small country with short distances to hospitals throughout the country, and existence of national guidelines. $^{34}$

The overall median time to RRSO of five to six months in both women affected and unaffected with breast cancer is equal or even a bit shorter compared to other studies. ${ }^{13,17}$ We observed a statistically significantly longer time to surgery of one month among BRCA1 mutation carriers aged 40 years or older. A possible explanation is that women who are diagnosed with a BRCA mutation after being tested because of a breast cancer diagnosis complete their breast cancer treatment first before undergoing RRSO.

We did not report on the uptake of risk-reducing mastectomy (RRM) in our study population because we decided to focus on RRSO. However, it is clear from our data that about 35\% of unaffected BRCA1/2 mutation carriers underwent RRM, which lies well within the broad range of uptake rates previously reported. ${ }^{9}$ The best explanation for this moderate uptake rate compared to the extremely high RRSO uptake is our less directive way of counseling in breast cancer risk management compared to EOC risk management because breast surveillance is a more or less equal alternative to RRM in terms of survival..$^{35}$

The main strength of our study is that we included a large cohort of consecutive women tested positively for a germline BRCA1/2 mutation in our center. By selection through our laboratory database, it is unlikely we missed any patients. Besides, our nationwide network and registry of histo- and cytopathology in the Netherlands (PALGA) allowed us to complete data on uptake of RRSO, regardless of in which Dutch hospital RRSO took place. We only might have missed a patient having undergone RRSO abroad. Therefore, the amounts of selection bias and missing data are very low in spite of the retrospective study design.

Some limitations have to be noted as well. At first, generalization may not be possible since our study is a single-center experience. Second, we did not discriminate between RRSO for purely preventive purposes and bilateral salpingo-oophorectomy possibly being part of breast cancer treatment and a preventive strategy for EOC at the same time. The latter might be even better accepted by BRCA1/2 mutation carriers and therefore it might have influenced the uptake of surgery.

An interesting population that we did not include in this study, consists of women from families with high occurrence of breast and ovarian cancer in absence of germline BRCA1/2 mutations. Before 2011, they were offered annual ovarian screening but we are not aware of their choices for RRSO after screening had been stopped in our center. Currently, these women are offered RRSO 
if they have at least two relatives with EOC: one first-degree and another first- or second-degree relative.

Future research should focus on long-term health consequences of (premenopausal) RRSO and how to minimize adverse effects, since the uptake of RRSO among BRCA1/2 mutation carriers at our Family Cancer Clinic is very high and, when possible, surgery is almost always undergone within the recommended age range. Minimizing long-term health consequences could be achieved by either optimizing hormone replacement therapy or by investigating alternative risk-reducing strategies in EOC risk management. A French study group is already investigating bilateral salpingectomy as alternative strategy restricted to women who are reluctant to standard RRSO (NCT01608074). However, this will not be feasible in our situation since our study shows that reluctance is highly exceptional in our population. Another study currently running in the U.S. compares ovarian cancer screening, RRSO, and salpingectomy with delayed oophorectomy (NCT01907789), which is neither applicable to our situation, since ovarian cancer screening is not routinely offered anymore. More applicable to our situation, we are currently comparing bilateral salpingectomy with delayed oophorectomy to RRSO in a preference trial (NCT02321228). ${ }^{36}$ 


\section{REFEREMCES}

1. Chen S, Parmigiani G. Meta-analysis of BRCA1 and BRCA2 penetrance. J Clin Oncol 2007; 25: 1329-33.

2. Hermsen BB, Olivier RI, Verheijen RH, et al. No efficacy of annual gynaecological screening in BRCA1/2 mutation carriers; an observational follow-up study. Br J Cancer 2007; 96: 1335-42.

3. Rebbeck TR, Kauff ND, Domchek SM. Meta-analysis of risk reduction estimates associated with riskreducing salpingo-oophorectomy in BRCA1 or BRCA2 mutation carriers. J Natl Cancer Inst 2009; 101: 80-7.

4. Marchetti C, De Felice F, Palaia I, et al. Risk-reducing salpingo-oophorectomy: a meta-analysis on impact on ovarian cancer risk and all cause mortality in BRCA 1 and BRCA 2 mutation carriers. BMC Womens Health 2014; 14: 150.

5. Antoniou A, Pharoah PD, Narod S, et al. Average risks of breast and ovarian cancer associated with BRCA1 or BRCA2 mutations detected in case Series unselected for family history: a combined analysis of 22 studies. Am J Hum Genet 2003; 72: 1117-30.

6. Guidelines NCCN. Genetic/familial high-risk assessment: breast and ovarian. 1.2015 ed. Washington, PA: National Comprehensive Cancer Network; 2015.

7. Walker JL, Powell CB, Chen LM, et al. Society of Gynecologic Oncology recommendations for the prevention of ovarian cancer. Cancer 2015.

8. Erfelijke tumoren: Richtlijnen voor diagnostiek en preventie. Vierde druk: Stichting Opsporing Erfelijke tumoren en Vereniging Klinische Genetica Nederland, Werkgroep Klinische Oncogenetica; 2010.

9. Hartmann LC, Lindor NM. The Role of Risk-Reducing Surgery in Hereditary Breast and Ovarian Cancer. N Engl J Med 2016; 374: 454-68.

10. Howard AF, Balneaves LG, Bottorff JL. Women's decision making about risk-reducing strategies in the context of hereditary breast and ovarian cancer: a systematic review. Journal of genetic counseling 2009; 18: $578-97$.

11. De Leeuw JR, van Vliet MJ, Ausems MG. Predictors of choosing life-long screening or prophylactic surgery in women at high and moderate risk for breast and ovarian cancer. Fam Cancer 2008; 7: 347-59.

12. Miller SM, Roussi P, Daly MB, Scarpato J. New strategies in ovarian cancer: uptake and experience of women at high risk of ovarian cancer who are considering risk-reducing salpingo-oophorectomy. Clin Cancer Res 2010; 16: 5094-106.

13. Beattie MS, Crawford B, Lin F, Vittinghoff E, Ziegler J. Uptake, time course, and predictors of risk-reducing surgeries in BRCA carriers. Genet Test Mol Biomarkers 2009; 13: 51-6.

14. Sidon L, Ingham S, Clancy T, et al. Uptake of risk-reducing salpingo-oophorectomy in women carrying a BRCA1 or BRCA2 mutation: evidence for lower uptake in women affected by breast cancer and older women. Br J Cancer 2012; 106: 775-9.

15. Kim SI, Lim MC, Lee DO, et al. Uptake of risk-reducing salpingo-oophorectomy among female BRCA mutation carriers: experience at the National Cancer Center of Korea. J Cancer Res Clin Oncol 2016; 142: 333-40.

16. Pal T, Lee JH, Besharat A, et al. Modes of delivery of genetic testing services and the uptake of cancer risk management strategies in BRCA1 and BRCA2 carriers. Clin Genet 2014; 85: 49-53.

17. Bradbury AR, Ibe CN, Dignam JJ, et al. Uptake and timing of bilateral prophylactic sal pingo-oophorectomy among BRCA1 and BRCA2 mutation carriers. Genet Med 2008; 10: 161-6.

18. Manchanda R, Burnell M, Abdelraheim A, et al. Factors influencing uptake and timing of risk reducing salpingo-oophorectomy in women at risk of familial ovarian cancer: a competing risk time to event analysis. BJOG 2012; 119: 527-36.

19. van Driel CM, de Bock GH, Arts HJ, et al. Stopping ovarian cancer screening in BRCA1/2 mutation carriers: Effects on risk management decisions \& outcome of risk-reducing salpingo-oophorectomy specimens. Maturitas 2015; 80: 318-22. 
20. van der Aa JE, Hoogendam JP, Butter ES, Ausems MG, Verheijen RH, Zweemer RP. The effect of personal medical history and family history of cancer on the uptake of risk-reducing salpingo-oophorectomy. Fam Cancer 2015; 14: 539-44.

21. Madalinska JB, van Beurden M, Bleiker EM, et al. Predictors of prophylactic bilateral salpingooophorectomy compared with gynecologic screening use in BRCA1/2 mutation carriers. J Clin Oncol 2007; 25: 301-7.

22. Meijers-Heijboer $\mathrm{H}$, Brekelmans $\mathrm{CT}$, Menke-Pluymers $\mathrm{M}$, et al. Use of genetic testing and prophylactic mastectomy and oophorectomy in women with breast or ovarian cancer from families with a BRCA1 or BRCA2 mutation. J Clin Oncol 2003; 21: 1675-81.

23. Dhar SU, Cooper HP, Wang T, et al. Significant differences among physician specialties in management recommendations of BRCA1 mutation carriers. Breast Cancer Res Treat 2011; 129: 221-7.

24. Den Heijer M, van Asperen CJ, Harris $\mathrm{H}$, et al. International variation in physicians' attitudes towards prophylactic mastectomy - comparison between France, Germany, the Netherlands and the United Kingdom. Eur J Cancer 2013; 49: 2798-805.

25. Julian-Reynier C, Eisinger F, Moatti JP, Sobol H. Physicians' attitudes towards mammography and prophylactic surgery for hereditary breast/ovarian cancer risk and subsequently published guidelines. European journal of human genetics: EJHG 2000; 8: 204-8.

26. Julian-Reynier CM, Bouchard LJ, Evans DG, et al. Women's attitudes toward preventive strategies for hereditary breast or ovarian carcinoma differ from one country to another: differences among English, French, and Canadian women. Cancer 2001; 92: 959-68.

27. Evans DG, Lalloo F, Ashcroft L, et al. Uptake of risk-reducing surgery in unaffected women at high risk of breast and ovarian cancer is risk, age, and time dependent. Cancer Epidemiol Biomarkers Prev 2009; 18: 2318-24.

28. Metcalfe KA, Birenbaum-Carmeli D, Lubinski J, et al. International variation in rates of uptake of preventive options in BRCA1 and BRCA2 mutation carriers. Int J Cancer 2008; 122: 2017-22.

29. Friebel TM, Domchek SM, Neuhausen SL, et al. Bilateral prophylactic oophorectomy and bilateral prophylactic mastectomy in a prospective cohort of unaffected BRCA1 and BRCA2 mutation carriers. Clin Breast Cancer 2007; 7: 875-82.

30. Laitman $Y$, Vaisman $Y$, Feldman $D$, et al. Rates of risk-reducing surgery in Israeli BRCA1 and BRCA2 mutation carriers. Clin Genet 2014; 85: 68-71.

31. This $\mathrm{P}$, de la Rochefordiere A, Savignoni A, et al. Breast and ovarian cancer risk management in a French cohort of 158 women carrying a BRCA1 or BRCA2 germline mutation: patient choices and outcome. Fam Cancer 2012; 11: 473-82.

32. Heemskerk-Gerritsen BA, Seynaeve C, van Asperen CJ, et al. Breast cancer risk after salpingooophorectomy in healthy BRCA1/2 mutation carriers: revisiting the evidence for risk reduction. J Nat/ Cancer Inst 2015; 107.

33. Eisinger F, Geller G, Burke W, Holtzman NA. Cultural basis for differences between US and French clinical recommendations for women at increased risk of breast and ovarian cancer. Lancet. 1999; 353: 919-20.

34. Skytte AB, Gerdes AM, Andersen MK, et al. Risk-reducing mastectomy and salpingo-oophorectomy in unaffected BRCA mutation carriers: uptake and timing. Clin Genet 2010; 77: 342-9.

35. Heemskerk-Gerritsen BA, Menke-Pluijmers MB, Jager A, et al. Substantial breast cancer risk reduction and potential survival benefit after bilateral mastectomy when compared with surveillance in healthy BRCA1 and BRCA2 mutation carriers: a prospective analysis. Ann Oncol 2013; 24: 2029-35.

36. Harmsen MG, Arts-de Jong M, Hoogerbrugge N, et al. Early salpingectomy (TUbectomy) with delayed oophorectomy to improve quality of life as alternative for risk-reducing salpingo-oophorectomy in BRCA1/2 mutation carriers (TUBA study): a prospective non-randomised multicentre study. BMC Cancer 2015; 15: 593. 


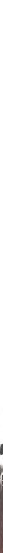

Peritoneal carcinomatosis after miskreducing surgery in BRCA1/2 mutation carriens: new implications for gynecologic counseling

Marline G. Harmsen, Jurgen M.J. Piek, Johan Bulten, Murray J. Casey, Timothy R. Rebbeck, Marian J.E. Mourits, Mark H. Greene, Brigitte F.M. Slangen, Marc van Beurden, Leon F.A.G. Massuger, Nicoline Hoogerbrugge, Joanne A. de Hullu 


\section{ABSTRACT}

\section{Background}

Risk-reducing salpingo-oophorectomy (RRSO) is recommended to BRCA1/2 mutation carriers because of their increased risk of ovarian carcinoma. Despite RRSO, metachronous peritoneal carcinomatosis is occasionally diagnosed. Knowledge regarding occurrence and pathogenesis is necessary to improve gynecologic counseling on risk-reducing surgery.

\section{Methods}

Literature was searched for publications describing BRCA1/2 mutation carriers with peritoneal carcinomatosis after risk-reducing surgery and authors were requested to provide additional data and cases. Descriptive statistics were used to analyze clinical and histopathological data and to compare cases with a single-institution control cohort.

\section{Results}

Thirty-one of 36 collected cases (86.1\%) concerned BRCA1 mutation carriers. Median age was 52 years (range 30-71) at risk-reducing surgery and 60 years (range 37-75) at peritoneal carcinomatosis diagnosis, and median interval between those two was 54.5 months (range 11-292). The vast majority of peritoneal carcinomatosis was of the high-grade serous subtype. Histopathological details of RRSO specimens including the complete fallopian tubes were retrieved in 8 cases: 5 (62.5\%) with serous tubal intraepithelial carcinoma (STIC), one with epithelial atypia. Cases were statistically significantly older $(p=0.025)$ at risk-reducing surgery and harbored more STICs $(p<0.001)$ than women from the control cohort.

\section{Conclusion}

Metachronous peritoneal carcinomatosis after risk-reducing surgery occurs predominantly in BRCA1 mutation carriers, within 5 years. Data suggest that surgery at younger age might lower rates of peritoneal carcinomatosis. These data can be used in gynecologic counseling of BRCA1/2 mutation carriers. RRSO should include complete salpingectomy, and detailed histopathological examination of specimens removed at the time of RRSO is essential. 


\section{InTRODUCTION}

Women carrying a germline mutation in one of the BRCA1 or BRCA2 genes have an increased risk of developing ovarian carcinoma.' Ovarian carcinoma is a collective term for carcinomas located in ovaries, fallopian tubes and/or peritoneum, and are of the high-grade serous histological subtype in about two thirds of reported BRCA-related cases., ${ }^{2,3}$

Given the poor prognosis of (high-grade serous) ovarian carcinoma and the lack of effective screening modalities, risk-reducing salpingo-oophorectomy (RRSO) is recommended between ages 35 and 40 years in BRCA1 mutation carriers and between ages 40 and 45 years in BRCA2 mutation carriers, prior to age-related increases in risk for this disease. This procedure reduces the risk of ovarian carcinoma by $80-96 \% .{ }^{4-6}$ However, the residual cumulative risk of peritoneal carcinoma, i.e., "peritoneal carcinomatosis", has been estimated to be 3.9\% for BRCA1 mutation carriers and $1.9 \%$ for BRCA2 mutation carriers in the 20 years following RRSO and might be lower when RRSO is performed at younger age. Currently, there are limited data that allow the prediction of which women will develop metachronous peritoneal carcinomatosis and when this cancer will occur.

Several sites have been postulated as possible origins of peritoneal carcinomatosis. ${ }^{8,9}$ Studies indicated that many or most cases originate from remnants of the embryonic Müllerian ducts, especially from fallopian tube epithelium, including the tubal-peritoneal junction. ${ }^{10-12}$ However, detection of precancerous lesions is not ubiquitous in completely sampled fallopian tubes of patients with peritoneal carcinomatosis, varying from 40-70\% in reported cases, ${ }^{13}$ leading others to propose that endometrial epithelium, another Müllerian derivative, also may be the origin of some peritoneal carcinomatosis. ${ }^{14,15}$ Ovarian surface epithelium and the "secondary Müllerian system" of pelvic and lower abdominal mesothelium and mesenchyme also are proposed as possible primary sites of malignant transformation. ${ }^{8}$ The latter two hypotheses are less likely due to frequent expression of Müllerian-specific rather than coelomic-specific markers by these cancer cells, ${ }^{16}$ although malignant transformation of mesothelium through Müllerian metaplasia cannot be ruled out.

Peritoneal carcinomatosis occurring many months after removal of the adnexa with or without hysterectomy in BRCA1/2 mutation carriers may have arisen from fallopian tube or endometrial epithelium cells shed into the pelvic cavity before that surgery. A similar mechanism is described for endometrial cells disseminated through retrograde menstruation. ${ }^{17}$ In like manner, ectopic cells from Müllerian epithelium may develop into a full-blown carcinoma, that may appear to be of peritoneal origin. If the interval is long between risk-reducing surgery and the presentation of peritoneal carcinoma, this hypothesis seems less plausible and another or at least a second pathway in the development of peritoneal carcinomatosis has to be considered.

The aim of our present study is to collect and analyze clinical and pathological data from cases of peritoneal carcinomatosis diagnosed after intended risk-reducing surgery accrued to several institutions across the world and transmit this information to inform gynecologic counseling of 
$B R C A 1 / 2$ mutation carriers. Some of these findings were compared with data gleaned from a recent single-institution cohort of BRCA1/2 mutation carriers who underwent RRSO at the Radboud university medical center, Nijmegen, The Netherlands.

\section{MATERIAL AMD METHODS}

\section{Data collection}

We performed a literature search in the Medline database to collect reported cases of peritoneal carcinomatosis after risk-reducing surgery in BRCA1/2 mutation carriers with no pathological abnormalities in the ovaries and fallopian tubes. The following search terms were used: $B R C A$, $B R C A 1, B R C A 2, H B O C$, familial, or high risk; risk-reducing surgery, salpingo-oophorectomy or oophorectomy; peritoneum, peritoneal or intra-abdominal; and cancer, cancers, carcinoma, carcinomas or carcinomatosis. The last date of search was December 31st, 2016. Studies describing one or more case(s) of peritoneal carcinomatosis after risk-reducing surgery were identified by scrutinizing titles and abstracts and full-texts. Both reference lists and citations of relevant papers were also checked to find articles we missed in the initial search. Only studies in English were included. Cases were excluded if either initial or revised microscopic evaluation of the salpingooophorectomy specimen revealed invasive carcinoma. Although we were only interested in $B R C A 1 / 2$ mutation carriers, studies that did not mention BRCA status or were published before the $B R C A$ genes had been identified were not excluded at this stage because of the possibility that the respective authors would have updated data on these cases.

Then, we contacted authors of the identified papers through email with the request to fill out a two-page case record form for each reported case and, if applicable, for other cases of peritoneal carcinomatosis after risk-reducing surgery from their experience. In the event they did not reply to our first request, we sent a reminder after four weeks and repeated if necessary. We also sent comparable emails to our national and international contacts who are experts in the field of hereditary breast and ovarian cancer.

The two-page case record form consisted of several items: age, BRCA mutation status, breast cancer history including stage and histology, type of risk-reducing surgery and histopathologic outcomes, details on peritoneal carcinomatosis diagnosis, e.g., age, histology, grade, stage and treatment, and the patient's vital status. All data were entirely anonymized before they were sent to us. We eliminated duplicates by comparing birth years and dates of surgeries. Local legal procedures of each contributing institution were followed to allow data sharing whenever required. Whenever $B R C A$ mutation status was unclear or negative, cases were excluded.

These findings were compared with a single-institution cohort of all BRCA1/2 mutation carriers who underwent RRSO at the Radboud university medical center between 2014 and 2016 and did not have invasive carcinoma in their surgical specimen $(\mathrm{N}=113)$. All RRSO specimens were completely 
embedded and sectioned according to the protocol for Sectioning and Extensively Examining the FIMbriated end (SEE-FIM), ${ }^{18}$ which has been adopted as institutional standard protocol. Precancerous lesions in the fallopian tube, including STICS, were classified using the algorithm published by Visvanathan et al. ${ }^{19}$ This cohort was used to analyze whether cases assembled from the literature of BRCA1/2 mutation carriers who developed metachronous peritoneal carcinomatosis after risk-reducing surgery had clinical characteristics different from this recent single-institution cohort of BRCA1/2 mutation carriers undergoing RRSO. It should be taken into account that all women from this recent cohort were recommended to undergo RRSO between 35 and 40 years of age for BRCA1 mutation carriers or between 40 and 45 years for BRCA2 mutation carriers, or as soon as possible in the event the germline mutation was detected at older age (41 years and older for $B R C A 1,46$ years and older for BRCA2).

The Medical Research Involving Human Subjects Act (Dutch: WMO) is not applicable to this study since it is a retrospective study using anonymized data from medical records. Therefore, the study was exempted from being approved by the Institutional Review Board.

\section{Statistical analysis}

We performed basic descriptive statistical analyses to summarize the retrieved set of international cases using the IBM SPSS Statistics version 22 software package. Median age at risk-reducing surgery, time interval between surgery and peritoneal carcinomatosis diagnosis, and survival were calculated. Distribution of histopathology, grade, and stage were summarized. The following tests were applied to compare cases with our single-institution cohort: Mann-Whitney test for nonparametrically distributed continuous variables, Chi-square test for categorical variables, and Fisher's Exact test for categorical variables in case of smaller subgroups.

\section{RESULTS}

Our Medline search yielded 129 articles, of which 21 remained after screening titles, abstracts, and full texts. An additional 7 relevant reports were identified after reference lists and citations were checked (Table 1). Contact information on the authors of one study describing one possibly relevant case could not be retrieved. ${ }^{20}$ Because some of the papers had overlapping authors, we eventually sent 20 email inquiries. Two authors replied that they did not have access to the requested data anymore and referred us to others, who subsequently did not reply to our emails. After several reminders, 6 filled out a total of 36 case record forms, of which 8 could be recognized from previous publications., ${ }^{51-23}$ Six cases could be added from our own institution. From Dutch colleagues, we received two additional cases. Email requests to several other international connections in the United Kingdom, Israel, Canada, Germany and Norway yielded no additional cases. 


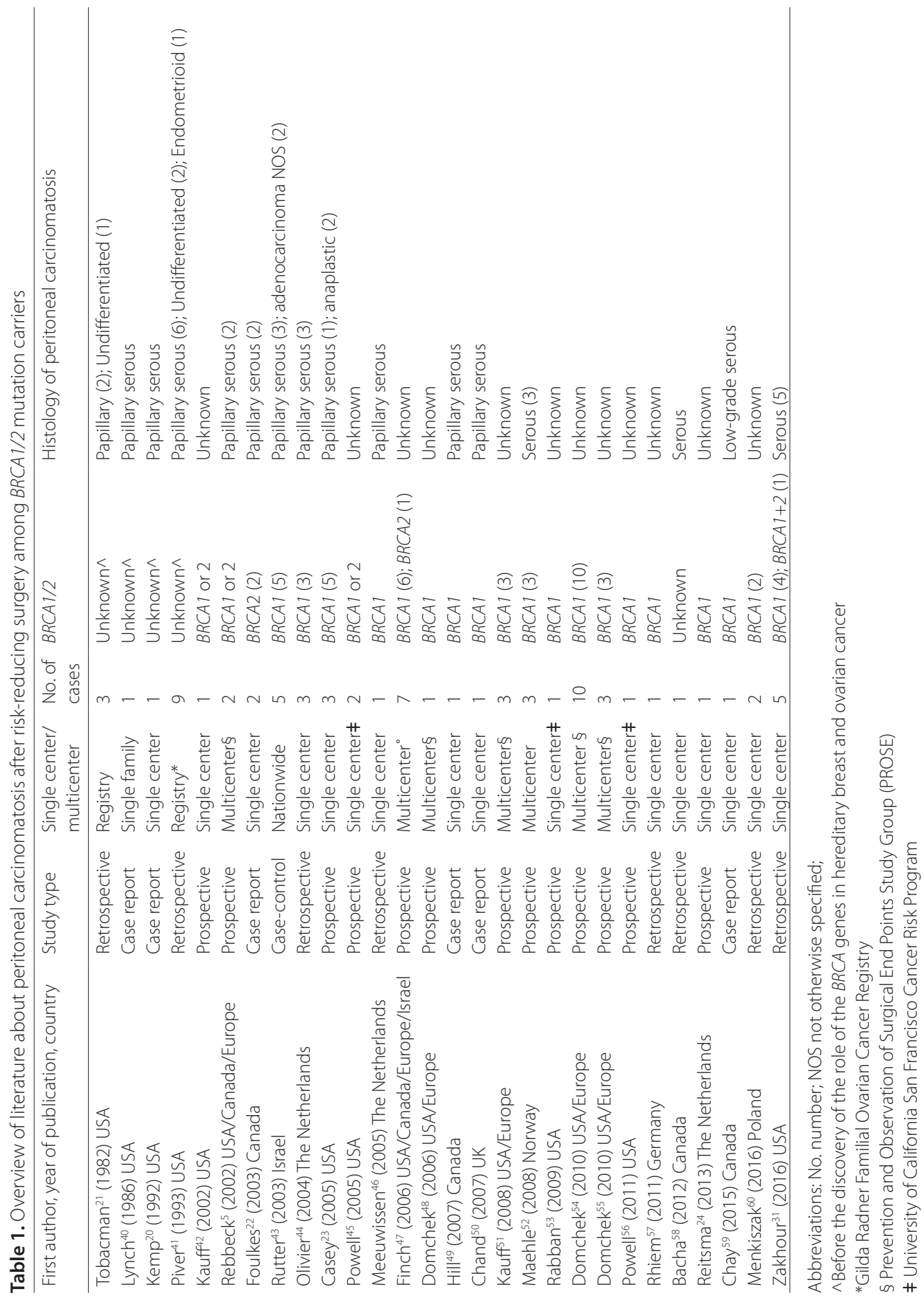


In sum, we collected 44 cases of peritoneal carcinomatosis after risk-reducing surgery without invasive carcinoma in BRCA1/2 mutation carriers. Of these, 8 were excluded because essential data were missing. No duplicates were detected and data from 36 cases could eventually be analyzed (Table 2).

Table 2. Sources of assembled cases of BRCA1/2 mutation carriers with peritoneal carcinomatosis after riskreducing surgery

\begin{tabular}{llll}
\hline Source & Country & Number of cases & $\begin{array}{l}\text { Previously published; } \\
\text { Number [ref] }\end{array}$ \\
\hline Creighton University School of Medicine & NE, USA & 16 (8 excluded) & $3^{23}$ \\
Dana Farber Cancer Institute & MA, USA & 9 & $1^{5}$ \\
Radboud university medical center & The Netherlands & 6 & 0 \\
University Medical Center Groningen & The Netherlands & 4 & 0 \\
Antoni van Leeuwenhoek hospital & The Netherlands & 3 & 0 \\
National Cancer Institute & MD, USA & 3 & $3^{21}$ \\
Maastricht University Medical Center & The Netherlands & 1 & 0 \\
Elisabeth-TweeSteden Hospital & The Netherlands & 1 & 0 \\
McGill university & Quebec, Canada & 1 & $1^{22}$ \\
\hline
\end{tabular}

Abbreviations: N/A not applicable;

Characteristics of assembled cases can be found in Table 3. Thirty-one of the 36 (86.1\%) assembled cases were from women who carried a BRCA1 germline mutation. The other 5/36 patients harbored a germline mutation in BRCA2. Risk-reducing surgeries took place between 1961 and 2014. The median age at surgery was 52 years (range 30-71, see Figure 1) for the whole group and statistically significantly differed between BRCA1 and BRCA2 mutation carriers: 51 years (range 30-71) and 57 years (range $56-65)$, respectively $(p=0.006)$. The median age that peritoneal carcinomatosis was diagnosed is 60 years (range 37-75), and the median interval between risk-reducing surgery and diagnosis of peritoneal carcinomatosis was 54.5 months (range 11-292 months, see Figure 2). Interval between risk-reducing surgery and peritoneal carcinomatosis was not related to age at risk-reducing surgery (see Figure 3). Most patients had undergone salpingo-oophorectomy (31/35, 86.1\%), and 16 (44.4\%) also had a hysterectomy. Three women underwent only oophorectomy, with most of the fallopian tubes left in situ according to the pathology report, although clinical notes suggested that total salpingo-oophorectomy was performed in two of them. In two other cases, it was unclear whether salpingectomy was performed. The complete surgical specimens including the fallopian tube of only 8 women from the assembled cases were known to be entirely embedded and examined. Five of these 8 women had STICS, and another showed tubal epithelial atypia. In most cases, however, ovaries and fallopian tubes were not entirely sampled so precancerous lesions and occult invasive cancers might have been missed. The median survival time was 24 months (range 2-80) after the diagnosis of peritoneal carcinomatosis in 19 patients known to have died of their disease. 


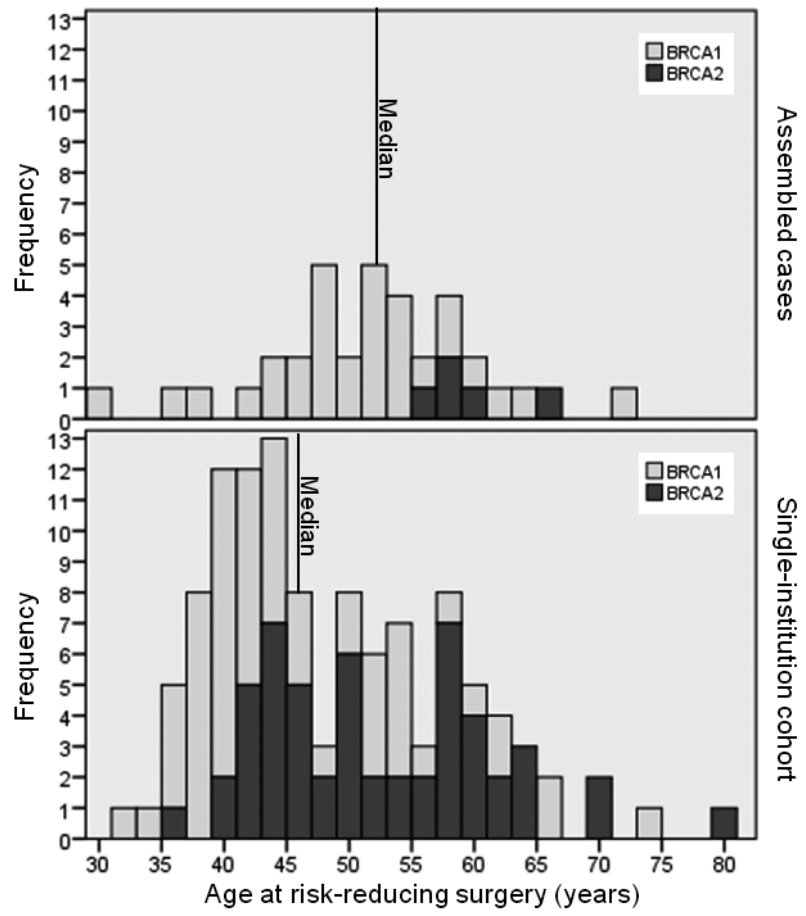

Figure 1. Distribution of age at risk-reducing surgery

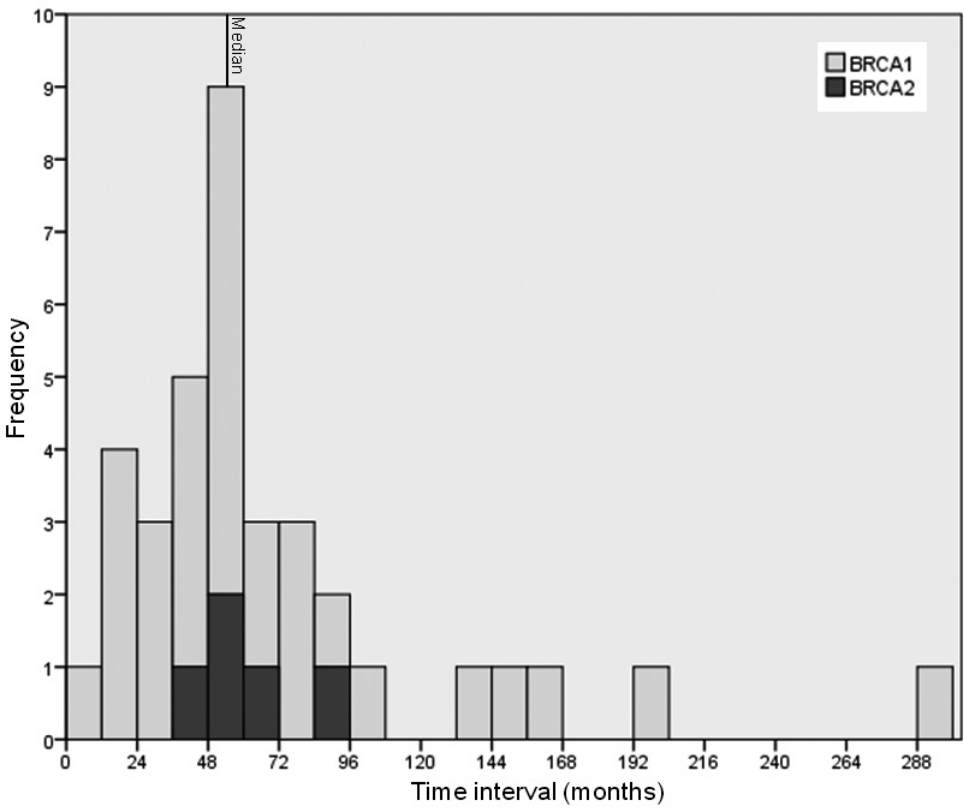

Figure 2. Distribution of interval between risk-reducing surgery and peritoneal carcinomatosis 


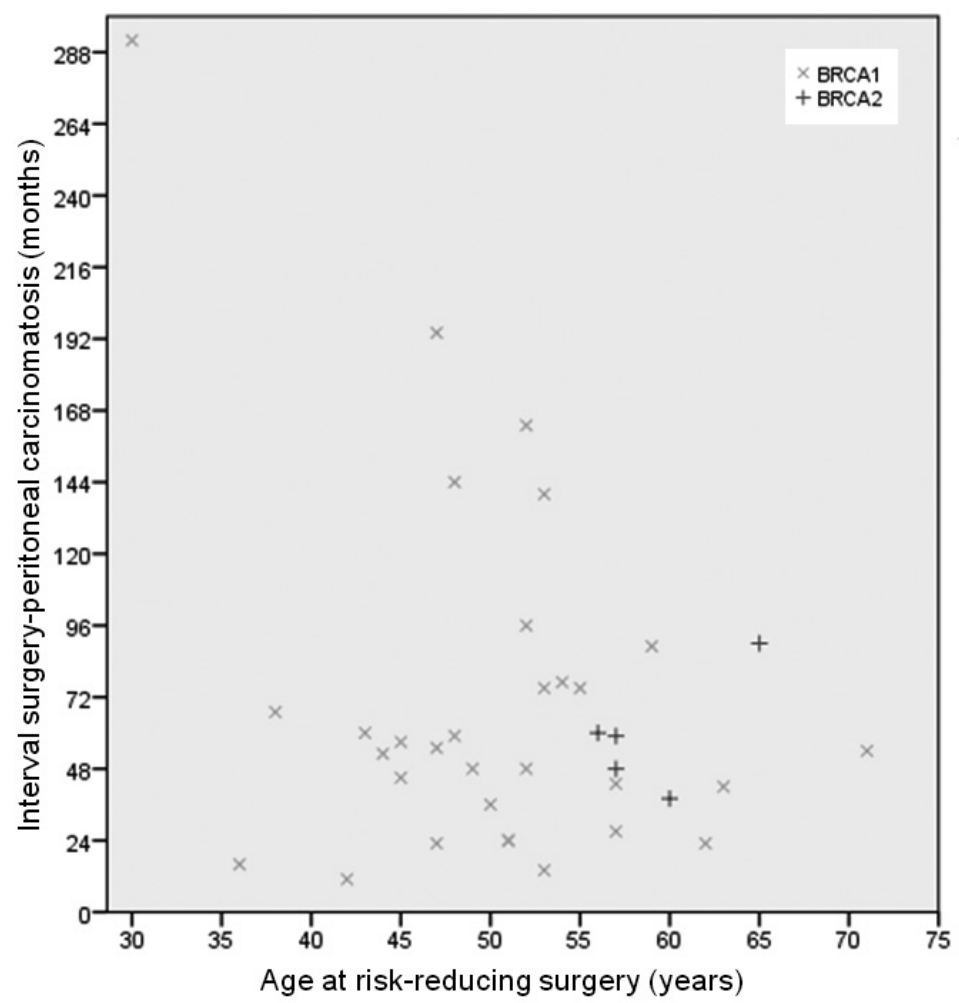

Figure 3. Relationship between age at risk-reducing surgery and time to peritoneal carcinomatosis diagnosis for 36 cases

Further details and the clinical data from the single-institution cohort $(\mathrm{N}=113)$ that underwent RRSO in the Radboud university medical center between 2014 and 2016 and were not found to have invasive carcinoma at RRSO are shown in Table 3. The following variables statistically significantly differed between cases of metachronous peritoneal carcinomatosis after risk-reducing surgery and those from the consecutive, single-institution cohort of women who underwent RRSO: median age at risk-reducing surgery 52 years (range 30-71) versus 46 years (range 32-80) ( $p=0.025$, Figure 1), proportion of BRCA1 mutation carriers $86.1 \%$ versus $53.1 \%(p<0.001)$, proportion of STICs in the RRSO specimen $62.5 \%$ versus $0 \%$ ( $p<0.001$ ) (Table 3 ). 
Table 3. Patient and tumor characteristics

\begin{tabular}{|c|c|c|c|}
\hline & $\begin{array}{l}\text { PC after } \mathrm{RR}(\mathrm{S}) \mathrm{O} ; \\
\text { cases; multicenter } \\
\mathrm{N}=36\end{array}$ & $\begin{array}{l}\text { Cohort RRSO; } \\
\text { single institution } \\
\mathrm{N}=113\end{array}$ & $p$-value \\
\hline Germline mutation & & & $<0.001^{* *}$ \\
\hline$B R C A 1$ & $31(86.1 \%)$ & $60(53.1 \%)$ & \\
\hline$B R C A 2$ & $5(13.9 \%)$ & $53(46.9 \%)$ & \\
\hline Previous breast cancer & & & $N S^{* *}$ \\
\hline Yes & $14(38.9 \%)$ & $35(31.0 \%)$ & \\
\hline No & $20(55.6 \%)$ & $78(69.0 \%)$ & \\
\hline Unknown & $2(5.5 \%)$ & 0 & \\
\hline Age at $\mathrm{RR}(\mathrm{S}) \mathrm{O}$ (median in years (range)) & $52(30-71)$ & $46(32-80)$ & $0.025^{* * *}$ \\
\hline \multicolumn{4}{|l|}{ Type of surgery } \\
\hline Bilateral oophorectomy & $36(100 \%)$ & $113(100 \%)$ & \\
\hline Bilateral salpingectomy & $31(86.1 \%)$ & $113(100 \%)$ & \\
\hline Unknown & $2(5.7 \%)$ & 0 & \\
\hline Hysterectomy (previous or simultaneous) & $16(44.4 \%)$ & $1(0.9 \%)$ & \\
\hline \multicolumn{4}{|l|}{$\mathrm{RR}(\mathrm{S}) \mathrm{O}$ specimen entirely examined } \\
\hline Yes* & $8(22.2 \%)$ & $113(100 \%)$ & \\
\hline Atypia in fallopian tube & $1(12.5 \%)$ & $9(8.0 \%)$ & NS^ \\
\hline STIC in fallopian tube & $5(62.5 \%)$ & 0 & $<0.001 \wedge$ \\
\hline No & $22(61.1 \%)$ & 0 & \\
\hline Unknown & $5(13.9 \%)$ & 0 & \\
\hline Time interval RR(S)O - PC (median in months (range)) & $54.5(11-292)$ & N/A & \\
\hline Age at PC diagnosis (median in years (range)) & $60(37-75)$ & N/A & \\
\hline Histology of PC & & N/A & \\
\hline Serous & $21(58.3 \%)$ & & \\
\hline Clear cell & $1(2.8 \%)$ & & \\
\hline Adenocarcinoma NOS & $4(11.1 \%)$ & & \\
\hline Undifferentiated & $2(5.6 \%)$ & & \\
\hline Unknown & $8(22.2 \%)$ & & \\
\hline Stage & & N/A & \\
\hline III & $18(50.0 \%)$ & & \\
\hline IV & $8(22.2 \%)$ & & \\
\hline Unknown & $10(27.8 \%)$ & & \\
\hline Grade & & $\mathrm{N} / \mathrm{A}$ & \\
\hline High (grade 2/3) & $24(66.7 \%)$ & & \\
\hline Low (grade 1) & $1(2.8 \%)$ & & \\
\hline Unknown & $11(30.6 \%)$ & & \\
\hline Deceased at last follow-up & & $\mathrm{N} / \mathrm{A}$ & \\
\hline Yes & $19(52.8 \%)$ & & \\
\hline $\begin{array}{l}\text { Duration from PC diagnosis to death } \\
\text { (median in months (range)) }\end{array}$ & $24(2-80)$ & & \\
\hline No & $15(41.7 \%)$ & & \\
\hline Unknown & $2(5.5 \%)$ & & \\
\hline
\end{tabular}

Abbreviations: PC peritoneal carcinomatosis; RR(S)O risk-reducing (salpingo-)oophorectomy; NS not significant; STIC serous tubal intraepithelial carcinoma; N/A not applicable; NOS not otherwise specified;

* Only the worst feature counted

** Chi square test

*** Mann-Whitney test

$\wedge$ Fisher's exact test 


\section{DIScussion}

Metachronous peritoneal carcinomatosis diagnosed in BRCA1/2 mutation carriers after riskreducing surgery is rare. However, every individual case is particularly dramatic because these patients did their utmost to prevent this lethal disease. In this study, we collected detailed clinical and pathological data on a relatively large series of such cases from centers in the Netherlands, Canada, and the United States. The majority of assembled cases were in BRCA1 mutation carriers with far fewer in BRCA2 mutation carriers. The median age at the time of risk-reducing surgery was younger for BRCA1 mutation carriers than for BRCA2 mutation carriers. The median age at the time of risk-reducing surgery was 52 years, the median age at peritoneal carcinomatosis was 60 years, and the median interval between those two was 54.5 months. Most of the risk-reducing surgeries in assembled cases were performed before 2005, an era when pathology specimens were not necessarily fully examined nor was it particularly noted whether or not fallopian tubes were excised, which was not standard of care at that time. Five of eight entirely examined surgical specimens from RRSOs in assembled cases contained STIC and another showed atypia in the tubal epithelium. These findings from the assembled cases differed significantly from our singleinstitution cohort, which consisted of BRCA1/2 mutation carriers with overall lower median age at the time of RRSO, a higher proportion of BRCA2 mutation carriers and no STIC or occult carcinomas detected in their surgical pathology specimens.

Finch et al. ${ }^{7}$ reported on cancer incidence in an international registry from 43 hospitals in 7 countries including 3513 BRCA1/2 mutation carriers who underwent risk-reducing surgery. Twenty-eight of 2649 BRCA 1 (1.1\%) and 4 of 864 BRCA2 mutation carriers (0.5\%) developed peritoneal carcinomatosis. The mean age at the time of risk-reducing surgery was not reported by these authors. The mean age at the time of diagnosis was 51.6 years (range 36-69 years), and the average interval after riskreducing surgery was 6.1 years (range 1-20 years), which are consistent with the findings from our assembled cases. Finch et al. estimated cumulative risks of peritoneal carcinomatosis of 3.9\% in BRCA1 and $1.9 \%$ in BRCA2 mutation carriers during the 20 years following risk-reducing surgery? Metachronous peritoneal carcinomatosis occurred in $0.3 \%$ of the women in this cadre who underwent risk-reducing surgery before the age of 40 years and in $0.7 \%$ of those who underwent surgery between 40 and 50 years. The median age that peritoneal carcinomatosis was diagnosed was higher and the median interval between risk-reducing surgery and this diagnosis was shorter in our study of assembled cases than the mean age and interval reported by Finch et al.? Although early RRSO seems beneficial in terms of lower incidence of STIC and metachronous peritoneal carcinomatosis, $7,24,25$ the latter still occurred after (complete) RRSO performed at 36 years in one case. Nevertheless, our data favor the current recommendation of risk-reducing surgery at 35-40 years for BRCA1 mutation carriers and 40-45 years for BRCA2 mutation carriers. ${ }^{26}$

Peritoneal carcinomas were high-grade serous in the vast majority of assembled cases (75\% of cases with known histology). One clear cell carcinoma was detected. The remaining were 
undifferentiated or unclassified carcinomas, consistent with histological findings of peritoneal carcinomatosis in other publications. ${ }^{27}$ However, the histological distribution may differ over time due to changes in histopathological classification guidelines over the years. Application of WHO 2014 criteria for classification shows that most ovarian carcinomas are HGSC, ${ }_{1}^{28}$ which now we expect is the appropriate classification for most of the peritoneal carcinomatosis in our assembled cases.

Our findings support an important role for the fallopian tube in the pathogenesis of peritoneal carcinomatosis by the appearance of STICS and the time interval after risk-reducing surgery. First, 5 STIC lesions were detected in 8 sufficiently pathologically examined RRSO specimens of women who subsequently developed peritoneal carcinomatosis (62.5\%), compared with no STICs in our control cohort and up to $8.0 \%$ in literature. ${ }^{24,29}$ This difference is notable, although the event numbers are small, and we cannot discern whether these peritoneal carcinomas were related to the initial STIC or had a different site of origin. Patrono et al..$^{30}$ reviewed the literature on the outcome of isolated STICs in RRSO specimens and found that 3 of 67 women (4.5\%), mainly BRCA1/2 mutation carriers, subsequently developed peritoneal carcinomatosis. Follow-up time ranged from 2 to 150 months. ${ }^{30} \mathrm{~A}$ more recent study from a single institution showed metachronous peritoneal carcinomatosis in 2 of 9 patients (22\%) with isolated STICs in their RRSO specimen, in contrast to only $1.2 \%$ of women with benign histology $(p=0.01) .{ }^{31}$ Interestingly, an identical TP53 mutation and a largely identical genomic imbalance were detected in a STIC from an RRSO specimen of a 45-year-old BRCA1 mutation carrier and peritoneal carcinoma that occurred ten years later in the same patient, suggesting a clonal origin. ${ }^{32}$ It remains debatable to what extent STICs should be considered cancer and treated by full staging or chemotherapy. ${ }^{33}$

The second finding supporting a role for the fallopian tube in peritoneal carcinomatosis pathogenesis is the relatively short time interval between prophylactic surgery and peritoneal carcinomatosis, i.e., 5 years or less in $66.7 \%$ of collected cases. According to a model for time of progression from occult fallopian and ovarian in situ and invasive carcinomas to clinically advanced intra-abdominal carcinoma constructed from published data by Brown and Palmer, ${ }^{34}$ the entire occult period of serous carcinoma is 5.1 years (median; 95\% confidence interval 3.2-8.1 years), so (pre)cancerous tubal epithelium cells that were shed into the abdominal cavity just before removal of fallopian tubes and ovaries could plausibly present as peritoneal carcinomatosis many years later. This may result in the latter being misdiagnosed as a "peritoneal" primary rather than being recognized as recurrent STIC or occult tubal carcinoma and this may mainly happen when (pre)malignancies were missed at incomplete histopathological examination of prophylactically removed fallopian tubes. Additionally, $98 \%$ of cases with metachronous peritoneal carcinomatosis after curatively treated colorectal carcinoma were diagnosed within 5 years (median 18 months; range 2.5-88 months). ${ }^{35}$ The same mechanism - tumor cells shedding intra-abdominally and implanting in the peritoneum - might be responsible for these colorectal cancer-related peritoneal metastases, a possibility supported by the observation that these are more common after colon carcinomas than after rectal carcinomas, the latter located outside the peritoneal cavity. ${ }^{35}$ 
Fourteen percent of the assembled cases were diagnosed more than 8 years after risk-reducing surgery. This interval exceeds the upper limit of 95\% confidence for the calculated occult period of serous carcinoma. ${ }^{34}$ Attempting to explain this, it is important to note that although clinical notes may state "bilateral salpingo-oophorectomy", the fallopian tube might not be entirely removed during risk-reducing surgery, as was so in at least three of our assembled cases, and in two cases remnants of fallopian tube fimbriae were recognized in subsequent peritoneal biopsies. Moreover, normal tubal epithelium might have ingrained into peritoneum prior to salpingectomy, remaining benign until later beginning malignant transformation several years after surgery.1113 Alternatively, another site of peritoneal carcinoma origin remains plausible, especially in cases diagnosed after longer intervals following complete salpingo-oophorectomy with or without hysterectomy.

The strength of this study lies in the collection of 36 cases of peritoneal carcinomatosis in BRCA1/2 mutation carriers after risk-reducing surgery with corroboration and expansion of data on these assembled cases through direct communication with the authors. In addition, we compared characteristics of the assembled cases with those of our single-institution cohort of BRCA1/2 mutation carriers who had RRSO with benign histology. This comparison suggests that patients who are BRCA1 mutation carriers, who are older at risk-reducing surgery, and who harbor STICs are predisposed to develop peritoneal carcinomatosis. Although follow-up of this cohort is short and a few could develop peritoneal carcinomatosis in the future, that number would supposedly be very low and would not substantially alter the characteristics of this control cohort. Nevertheless, we have to take into account that this cohort has been counseled and treated according to the latest insights, while risk-reducing surgeries of 25/36 assembled cases were performed more than 10 years ago. Therefore, the comparison should be interpreted with caution. Furthermore, the absence of any STICs in our single-institution cohort of 113 BRCA1/2 mutation carriers is remarkable although incidences of $1 \%$ or less have been reported elsewhere. ${ }^{24,36,37}$ A possible explanation for the absence of STICS in our single-institution cohort is our pathologists' strict adherence to the algorithm published by Visvanathan et al. ${ }^{19}$ to classify precancerous lesions of the fallopian tube. In this algorithm, both a TP53 mutated immunohistochemical profile and Ki-67 positivity are required for the diagnosis of STIC. Application of these criteria to other studies reporting on STIC incidence would lower the incidence, e.g., only 1 of 8 isolated STICs detected in another study fulfilled these criteria, thereby decreasing the incidence from $10.4 \%$ to $1.3 \%{ }^{25}$ Other studies did not report the use of TP53 and Ki-67 immunohistochemistry. ${ }^{24,29,36}$

This study has several limitations. In some cases, particularly those reported long ago, significant amounts of data were missing from the clinical records. With but few exceptions, the SEE-FIM protocol was not employed to examine pathology specimens from risk-reducing surgeries in many of our assembled cases of peritoneal carcinomatosis in BRCA1/2 mutation carriers, as the surgeries antedated widespread adoption of this now standard method for pathological processing..$^{18}$ During that era, the role of the fallopian tube in "ovarian"carcinogenesis had not been recognized, and many risk-reducing oophorectomies did not specifically remove the fallopian tubes. Because complete diagnostic tissues were irretrievable in most of the assembled cases, centralized pathological review 
was not done on either prophylactic surgery specimens or peritoneal carcinomatosis. Moreover, selection bias to a certain extent occurred because we asked professionals to send cases from their own practice. Remarkable cases are more likely to be remembered, e.g., peritoneal carcinomatosis after a previous STIC in the RRSO specimen, which might explain the high proportion of STICS among assembled cases. Additionally, metachronous peritoneal carcinomatosis might be a reason to meticulously revise histopathology of RRSO specimens thereby increasing the chance of finding STICS. Altogether, our findings cannot be fully generalized to patients coming through health services today. Lastly, we did not collect details on mutation location. It would be very interesting to see whether women with metachronous peritoneal carcinomatosis after RRSO harbor specific mutations in the ovarian cancer cluster regions, for instance. . $^{3,39}$

In conclusion, the majority of peritoneal carcinomatosis cases were diagnosed fewer than 5 years after risk-reducing surgery in the 36 assembled cases, and these were mostly in women who underwent surgery later than the currently recommended ages of 35-40 years for BRCA1 mutation carriers and 40-45 years for BRCA2 mutation carriers. These findings can be used to refine gynecologic counseling of BRCA1/2 mutation carriers who consider risk-reducing surgery and to stress the importance of complete RRSO at the recommended ages. Complete examination according to the SEE-FIM protocol should be followed in all pathological processing of surgical specimens from BRCA1/2 mutation carriers; albeit, as yet, there is no consensus as to how patients should be managed when preinvasive lesions are found. In the quest for better understanding of pathogenesis and to address outstanding questions for counseling and clinical management, further prospective research is needed using agreed standards for the delivery and processing of surgical specimens from BRCA1/2 mutation carriers and uniform formats and nomenclature for reporting demographic, medical, surgical and pathology data with long-term follow-up to specialized central cancer registries.

\section{Acknowledgments}

The authors would like to thank Professor William D. Foulkes from the McGill university in Montreal, Quebec, Canada, and Mrs. M. Caroline Vos from the Elisabeth-Tweesteden hospital in Tilburg, the Netherlands, for kindly sharing their data, and Carrie Snyder from the Hereditary Cancer Prevention Clinic at Creighton University for her technical assistance with data collection. 


\section{REFEREMCES}

1. Chen S, Parmigiani G. Meta-analysis of BRCA1 and BRCA2 penetrance. J Clin Oncol 2007; 25(11): 1329-33.

2. Mavaddat N, Barrowdale D, Andrulis IL, et al. Pathology of breast and ovarian cancers among BRCA1 and BRCA2 mutation carriers: results from the Consortium of Investigators of Modifiers of BRCA1/2 (CIMBA). Cancer Epidemiol Biomarkers Prev 2012; 21 (1): 134-47.

3. Bolton KL, Chenevix-Trench G, Goh C, et al. Association between BRCA1 and BRCA2 mutations and survival in women with invasive epithelial ovarian cancer. JAMA : the journal of the American Medical Association 2012; 307(4): 382-90.

4. Rebbeck TR, Kauff ND, Domchek SM. Meta-analysis of risk reduction estimates associated with riskreducing salpingo-oophorectomy in BRCA1 or BRCA2 mutation carriers. J Natl Cancer Inst 2009; 101(2): 80-7.

5. Rebbeck TR, Lynch HT, Neuhausen SL, et al. Prophylactic oophorectomy in carriers of BRCA1 or BRCA2 mutations. N Engl J Med 2002; 346(21): 1616-22.

6. Marchetti C, De Felice F, Palaia I, et al. Risk-reducing salpingo-oophorectomy: a meta-analysis on impact on ovarian cancer risk and all cause mortality in BRCA 1 and BRCA 2 mutation carriers. BMC Womens Health 2014; 14(1): 150.

7. Finch AP, Lubinski J, Moller P, et al. Impact of oophorectomy on cancer incidence and mortality in women with a BRCA1 or BRCA2 mutation. J Clin Oncol 2014; 32(15): 1547-53.

8. Singh N, Gilks CB, Wilkinson N, McCluggage WG. The secondary Mullerian system, field effect, BRCA, and tubal fimbria: our evolving understanding of the origin of tubo-ovarian high-grade serous carcinoma and why assignment of primary site matters. Pathology 2015; 47(5): 423-31.

9. Nik NN, Vang R, Shih le M, Kurman RJ. Origin and pathogenesis of pelvic (ovarian, tubal, and primary peritoneal) serous carcinoma. Annu Rev Pathol 2014; 9: 27-45.

10. Seidman JD, Zhao P, Yemelyanova A. "Primary peritoneal" high-grade serous carcinoma is very likely metastatic from serous tubal intraepithelial carcinoma: assessing the new paradigm of ovarian and pelvic serous carcinogenesis and its implications for screening for ovarian cancer. Gynecol Oncol 2011; 120(3): 470-3.

11. Carlson JW, Miron A, Jarboe EA, et al. Serous tubal intraepithelial carcinoma: its potential role in primary peritoneal serous carcinoma and serous cancer prevention. J Clin Oncol 2008; 26(25): 4160-5.

12. Piek JM, Kenemans $P$, Verheijen RH. Intraperitoneal serous adenocarcinoma: a critical appraisal of three hypotheses on its cause. Am J Obstet Gynecol 2004; 191(3): 718-32.

13. Horn LC, Kafkova S, Leonhardt K, Kellner C, Einenkel J. Serous tubal in situ carcinoma (STIC) in primary peritoneal serous carcinomas. Int J Gynecol Pathol 2013; 32(4): 339-44.

14. Roelofsen T, van Kempen LC, van der Laak JA, van Ham MA, Bulten J, Massuger LF. Concurrent endometrial intraepithelial carcinoma (EIC) and serous ovarian cancer: can EIC be seen as the precursor lesion? Int J Gynecol Cancer 2012; 22(3): 457-64.

15. Mingels MJ, van Ham MA, de Kievit IM, et al. Mullerian precursor lesions in serous ovarian cancer patients: using the SEE-Fim and SEE-End protocol. Mod Pathol 2014; 27 (7): 1002-13.

16. Tong GX, Chiriboga L, Hamele-Bena D, Borczuk AC. Expression of PAX2 in papillary serous carcinoma of the ovary: immunohistochemical evidence of fallopian tube or secondary Mullerian system origin? Mod Pathol 2007; 20(8): 856-63.

17. Nap AW, Groothuis PG, Demir AY, Evers JL, Dunselman GA. Pathogenesis of endometriosis. Best Pract Res Clin Obstet Gynaecol 2004; 18(2): 233-44.

18. Medeiros F, Muto MG, Lee $Y$, et al. The tubal fimbria is a preferred site for early adenocarcinoma in women with familial ovarian cancer syndrome. Am J Surg Pathol 2006; 30(2): 230-6.

19. Visvanathan K, Vang R, Shaw P, et al. Diagnosis of serous tubal intraepithelial carcinoma based on morphologic and immunohistochemical features: a reproducibility study. Am J Surg Pathol 2011; 35(12): 1766-75. 
20. Kemp GM, Hsiu JG, Andrews MC. Papillary peritoneal carcinomatosis after prophylactic oophorectomy. Gynecol Oncol 1992; 47(3): 395-7.

21. Tobacman JK, Greene MH, Tucker MA, Costa J, Kase R, Fraumeni JF, Jr. Intra-abdominal carcinomatosis after prophylactic oophorectomy in ovarian-cancer-prone families. Lancet 1982; 2(8302): 795-7.

22. Foulkes WD, Narod SA, Swenerton K, Panabaker K, Gilbert L. Re: Gynecologic surgeries and risk of ovarian cancer in women with BRCA1 and BRCA2 Ashkenazi founder mutations: an Israeli population-based case-control study. J Natl Cancer Inst 2003; 95(21): 1640.

23. Casey MJ, Synder C, Bewtra C, Narod SA, Watson P, Lynch HT. Intra-abdominal carcinomatosis after prophylactic oophorectomy in women of hereditary breast ovarian cancer syndrome kindreds associated with BRCA1 and BRCA2 mutations. Gynecol Oncol 2005; 97 (2): 457-67.

24. Reitsma W, de Bock GH, Oosterwijk JC, Bart J, Hollema H, Mourits MJ. Support of the 'fallopian tube hypothesis' in a prospective series of risk-reducing salpingo-oophorectomy specimens. Eur J Cancer 2013; 49(1): 132-41

25. Cass I, Walts AE, Barbuto D, Lester J, Karlan B. A cautious view of putative precursors of serous carcinomas in the fallopian tubes of BRCA mutation carriers. Gynecol Oncol 2014; 134(3): $492-7$.

26. Integraal Kankercentrum Nederland. Richtlijn Erfelijk en Familiair Ovariumcarcinoom (English: Guideline Hereditary and Familial Ovarian Carcinoma). 06-15-2015. http://www.oncoline.nl/erfelijk-en-familiairovariumcarcinoom (accessed 09-23-2016.

27. Barda G, Menczer J, Chetrit A, et al. Comparison between primary peritoneal and epithelial ovarian carcinoma: a population-based study. Am J Obstet Gynecol 2004; 190(4): 1039-45.

28. Kommoss S, Gilks CB, du Bois A, Kommoss F. Ovarian carcinoma diagnosis: the clinical impact of 15 years of change. Br J Cancer 2016; 115(8): 993-9.

29. Mingels MJ, Roelofsen T, van der Laak JA, et al. Tubal epithelial lesions in salpingo-oophorectomy specimens of BRCA-mutation carriers and controls. Gynecol Oncol 2012; 127(1): 88-93.

30. Patrono MG, Iniesta MD, Malpica A, et al. Clinical outcomes in patients with isolated serous tubal intraepithelial carcinoma (STIC): A comprehensive review. Gynecol Oncol 2015; 139(3): 568-72.

31. Zakhour M, Danovitch Y, Lester J, et al. Occult and subsequent cancer incidence following risk-reducing surgery in BRCA mutation carriers. Gynecol Oncol 2016; 143(2): 231-5.

32. Ewing-Graham PC, Roes EM, Dinjens WNM. Peritoneal Serous Carcinoma 10 years after Risk-reducing Bilateral Salpingo-oophorectomy. Pathologendagen (Pathology conference). Zeist, the Netherlands; 2015.

33. Chen LM. STIC-ing with what we know. Gynecol Oncol 2016; 143(2): 227-8.

34. Brown PO, Palmer C. The preclinical natural history of serous ovarian cancer: defining the target for early detection. PLoS Med 2009; 6(7): e1000114.

35. van Gestel YR, Thomassen I, Lemmens VE, et al. Metachronous peritoneal carcinomatosis after curative treatment of colorectal cancer. Eur J Surg Oncol 2014; 40(8): 963-9.

36. Sherman ME, Piedmonte M, Mai PL, et al. Pathologic findings at risk-reducing salpingo-oophorectomy: primary results from Gynecologic Oncology Group Trial GOG-0199. J Clin Oncol 2014; 32(29): 3275-83.

37. Finch A, Shaw P, Rosen B, Murphy J, Narod SA, Colgan TJ. Clinical and pathologic findings of prophylactic salpingo-oophorectomies in 159 BRCA1 and BRCA2 carriers. Gynecol Oncol 2006; 100(1): 58-64.

38. Rebbeck TR, Mitra N, Wan F, et al. Association of type and location of BRCA1 and BRCA2 mutations with risk of breast and ovarian cancer. JAMA : the journal of the American Medical Association 2015; 313(13): 1347-61.

39. Teixeira N, Mourits MJ, Vos JR, et al. Ovarian cancer in BRCA1/2 mutation carriers: The impact of mutation position and family history on the cancer risk. Maturitas 2015; 82 (2): 197-202.

40. Lynch HT, Bewtra C, Lynch JF. Familial ovarian carcinoma. Clinical nuances. Am J Med 1986; 81 (6): 1073-6.

41. Piver MS, Jishi MF, Tsukada Y, Nava G. Primary peritoneal carcinoma after prophylactic oophorectomy in women with a family history of ovarian cancer. A report of the Gilda Radner Familial Ovarian Cancer Registry. Cancer 1993; 71(9): 2751-5. 
42. Kauff ND, Satagopan JM, Robson ME, et al. Risk-reducing salpingo-oophorectomy in women with a BRCA1 or BRCA2 mutation. N Engl J Med 2002; 346(21): 1609-15.

43. Rutter JL, Wacholder S, Chetrit A, et al. Gynecologic surgeries and risk of ovarian cancer in women with BRCA1 and BRCA2 Ashkenazi founder mutations: an Israeli population-based case-control study. J Natl Cancer Inst 2003; 95(14): 1072-8.

44. Olivier RI, van Beurden M, Lubsen MA, et al. Clinical outcome of prophylactic oophorectomy in BRCA1/ BRCA2 mutation carriers and events during follow-up. Br J Cancer 2004; 90(8): 1492-7.

45. Powell CB, Kenley E, Chen LM, et al. Risk-reducing salpingo-oophorectomy in BRCA mutation carriers: role of serial sectioning in the detection of occult malignancy. J Clin Oncol 2005; 23(1): 127-32.

46. Meeuwissen PA, Seynaeve C, Brekelmans CT, Meijers-Heijboer HJ, Klijn JG, Burger CW. Outcome of surveillance and prophylactic salpingo-oophorectomy in asymptomatic women at high risk for ovarian cancer. Gynecol Oncol 2005; $97(2)$ : 476-82.

47. Finch A, Beiner M, Lubinski J, et al. Salpingo-oophorectomy and the risk of ovarian, fallopian tube, and peritoneal cancers in women with a BRCA1 or BRCA2 Mutation. JAMA : the journal of the American Medical Association 2006; 296(2): 185-92.

48. Domchek SM, Friebel TM, Neuhausen SL, et al. Mortality after bilateral salpingo-oophorectomy in BRCA1 and BRCA2 mutation carriers: a prospective cohort study. Lancet Oncol 2006; 7(3): 223-9.

49. Hill KA, Rosen B, Shaw P, Causer PA, Warner E. Incidental MRI detection of BRCA1-related solitary peritoneal carcinoma during breast screening--A case report. Gynecol Oncol 2007; 107(1): 136-9.

50. Chand M, Moore PJ, Clarke AD, Nash GF, HickiskT. A diagnostic dilemma following risk-reducing surgery for BRCA1 mutation - a case report of primary papillary serous carcinoma presenting as sigmoid cancer. World J Surg Oncol 2007; 5: 102.

51. Kauff ND, Domchek SM, Friebel TM, et al. Risk-reducing salpingo-oophorectomy for the prevention of BRCA1- and BRCA2-associated breast and gynecologic cancer: a multicenter, prospective study. J Clin Oncol 2008; 26(8): 1331-7.

52. Maehle L, Apold J, Paulsen T, et al. High risk for ovarian cancer in a prospective series is restricted to BRCA1/2 mutation carriers. Clin Cancer Res 2008; 14(22): 7569-73.

53. Rabban JT, Krasik E, Chen LM, Powell CB, Crawford B, Zaloudek CJ. Multistep level sections to detect occult fallopian tube carcinoma in risk-reducing salpingo-oophorectomies from women with BRCA mutations: implications for defining an optimal specimen dissection protocol. Am J Surg Pathol 2009; 33(12): 1878-85.

54. Domchek SM, Friebel TM, Singer CF, et al. Association of risk-reducing surgery in BRCA1 or BRCA2 mutation carriers with cancer risk and mortality. JAMA : the journal of the American Medical Association 2010; 304(9): 967-75.

55. Domchek SM, Friebel TM, Garber JE, et al. Occult ovarian cancers identified at risk-reducing salpingooophorectomy in a prospective cohort of BRCA1/2 mutation carriers. Breast Cancer Res Treat 2010; 124(1): 195-203.

56. Powell CB, Chen LM, McLennan J, et al. Risk-reducing salpingo-oophorectomy (RRSO) in BRCA mutation carriers: experience with a consecutive series of 111 patients using a standardized surgical-pathological protocol. Int J Gynecol Cancer 2011; 21(5): 846-51.

57. Rhiem K, Foth D, Wappenschmidt B, et al. Risk-reducing salpingo-oophorectomy in BRCA1 and BRCA2 mutation carriers. Arch Gynecol Obstet 2011; 283(3): 623-7.

58. Bacha OM, Gregoire J, Grondin K, et al. Effectiveness of risk-reducing salpingo-oophorectomy in preventing ovarian cancer in a high-risk French Canadian population. Int J Gynecol Cancer 2012; 22(6): 974-8.

59. Chay WY, Horlings HM, Tinker AV, Gelmon KA, Gilks CB. Low grade serious carcinoma of the peritoneum in a BRCA1 carrier previously diagnosed with a "low-grade serous tubal intra-epithelial carcinoma" (STIC) on risk reducing surgery. Gynecologic oncology reports 2015; 12: 72-4.

60. Menkiszak J, Chudecka-Glaz A, Gronwald J, et al. Prophylactic salpingo-oophorectomy in BRCA1 mutation carriers and postoperative incidence of peritoneal and breast cancers. J Ovarian Res 2016; 9 : 11. 

$\therefore$ (5)

Risk-reducing salpingectomy with delayed oophorectomy in BRCA1/2 mutation carriens: patients' and professionals' perspectives

Marieke Arts-de Jong, Marline G. Harmsen, Nicoline Hoogerbrugge, Leon F.A.G. Massuger, Rosella P.M.G. Hermens, Joanne A. de Hullu 


\section{ABSTRACT}

\section{Objective}

To identify influencing factors of BRCA1/2 mutation carriers and their professionals for risk-reducing salpingectomy (RRS) with delayed oophorectomy (RRO) as a substitute for risk-reducing salpingooophorectomy (RRSO) and for study participation on this concept.

\section{Methods}

A qualitative study was performed by four focus group interviews with 39 BRCA1/2 mutation carriers and semi-structured in-depth interviews with 23 professionals in the field of hereditary cancer. We used a theoretical framework of determinants of innovation within healthcare organizations to classify influencing factors (barriers and facilitators).

\section{Results}

Among BRCA1/2 mutation carriers, main barriers for RRS with delayed RRO were seriousness of ovarian cancer, family history, and previous breast cancer. Among professionals, delay of riskreducing effect of oophorectomy on breast cancer risk and a second operation were recognized as main barriers. Both BRCA1/2 mutation carriers and professionals found uncertainty about the effect of RRS with delayed RRO and ease of the decision to undergo RRSO important barriers. The main facilitator mentioned by both was longer maintenance of ovarian function thereby delaying negative effects of early surgical menopause. For study participation, BRCA1/2 mutation carriers mentioned a randomized study design as the main barrier, whereas professionals identified two facilitators, namely willingness of BRCA1/2 mutation carriers for study participation and uniform counseling. Furthermore, most BRCA1/2 mutation carriers and professionals were willing to consider participation in a future non-randomized study.

\section{Conclusions}

We identified several barriers and facilitators for RRS with delayed RRO, and for study participation which can be addressed to optimize the design and implementation of a non-randomized study. 


\section{InTRODUCTION}

Epithelial ovarian cancer is the most lethal gynecological malignancy. More than 10\% of all epithelial ovarian cancers are inherited, mostly caused by a mutation in the BRCA1 or BRCA2 genes. ${ }^{1-3}$ The lifetime risk of developing ovarian cancer at age 70 in women harboring a BRCA1 or BRCA2 mutation varies between $31-40 \%$ and $6-18 \%$, respectively, 4,5 while the risk in the general population is $1.7 \%$.

Therefore, risk-reducing salpingo-oophorectomy (RRSO) is recommended to BRCA1/2 mutation carriers around the age of 40 years. RRSO reduces the risk of ovarian cancer by $80-90 \%$. The earlier assumption that performing RRSO at premenopausal age reduces breast cancer incidence by half6,7 has recently became arguable., Besides the benefits of RRSO, concerns are growing regarding the adverse effects associated with early surgical menopause. Short-term effects of premature menopause are vasomotor and sexual symptoms, which may not be fully alleviated by the use of hormone therapy. ${ }^{10,11}$ Long-term effects include risk of osteoporosis, cardiovascular diseases, and cognitive impairment. ${ }^{12-14}$ Approximately $75 \%$ of all ovarian cancers diagnosed in BRCA1/2 mutation carriers are high-grade serous cancer of the ovaries, fallopian tubes or peritoneum. ${ }^{15}$ Based on recent scientific insights, a significant majority of high-grade serous ovarian cancer arise from the fallopian tube rather than the ovarian surface epithelium. ${ }^{16-23}$ Along with the side effects of early menopause, increasing attention is paid to a possible new strategy of risk-reducing salpingectomy (RRS) with delayed risk-reducing oophorectomy (RRO) in BRCA1/2 mutation carriers. ${ }^{24-26}$

To design an appropriate clinical trial to evaluate the safety of RRS with delayed RRO and its added value on not cancer-related morbidity and mortality is an interesting challenge. However, a broadly based support from both BRCA1/2 mutation carriers and professionals in this field is needed to give a potential study an optimal chance. Holman et al. ${ }^{27}$ recently performed an online survey in 204 premenopausal BRCA1/2 mutation carriers to study the acceptability of, and interest in a clinical trial of RRS with delayed RRO. They reported that 34\% were interested in a study on this subject, $35 \%$ were unsure, and 31\% were not interested.

To our knowledge, no qualitative data are yet available on the interest in RRS with delayed RRO and the willingness of both BRCA1/2 mutation carriers and their professionals to participate in a study on this subject. Therefore, our objective was to assess factors of influence, both at BRCA1/2 mutation carrier and healthcare provider level, for the choice of RRSO or RRS with delayed RRO, and for willingness to participate in a study on this subject. 


\section{MATERIALS AMD METHODS}

\section{Study design and setting}

A qualitative study was performed with BRCA1/2 mutation carriers and their treating professionals using explorative semi-structured interviews in group and individual setting, respectively. We opted for semi-structured interviews to let the participants talk freely with structured guidance from the interviewer using an interview guide. For BRCA1/2 mutation carriers, we chose a group setting as we expected that this is useful to obtain detailed information about personal and group feelings, perceptions and opinions related to RRS with delayed RRO. We opted for individual interviews with professionals to give everyone the opportunity to openly speak one's mind on this subject without the risk of mutual influence or mainly politically correct answers.

In The Netherlands, BRCA1/2 mutation analysis is concentrated in eight university hospitals. After the diagnosis of a BRCA1/2 mutation, women are generally referred to a surgeon/oncologist and gynecologist in a university hospital for information on risk-reducing strategies for breast and ovarian cancer. Surveillance could take place in either university or non-university hospitals.

\section{Study population}

\section{BRCA1/2 mutation carriers}

BRCA1/2 mutation carriers were recruited from the Radboud University Medical Center (Radboudumc) in Nijmegen, The Netherlands. Four focus group interviews were performed with partly different constitution. Three focus groups consisted of BRCA1/2 mutation carriers who had already undergone RRSO. Further selection in these three groups occurred on breast cancer history; one group of women without breast cancer, one group of women with prior breast cancer, and one mixed group of women with and without a history of breast cancer. The fourth group included younger women without a history of breast cancer who had not yet undergone RRSO.

\section{Professionals}

Six gynecological oncologists from eight university hospitals and one gynecological oncologist from a non-university hospital, all with special interest for hereditary cancer, were asked to participate in this study. None of the gynecological oncologists of our own institute were included, because they initiated this study. All seven gynecological oncologists were asked to propose collaborative professionals from their department of clinical genetics and breast surveillance for study participation. Seven clinical geneticists from seven university hospitals were interviewed; nine professionals taking care of breast surveillance participated, Of which five surgeons, one medical oncologist, two medical doctors, and one nurse practitioner. 


\section{Data collection}

BRCA1/2 mutation carriers

Invitations for the first three focus group interviews were sent by email to $45 B R C A 1 / 2$ mutation carriers who had given written consent to be contacted for focus group research in the past. For the fourth focus group, we approached 21 BRCA1/2 mutation carriers through a letter with information about the study. After one week, we contacted them by phone to answer any questions and to ask for consent to participate.

The interview guide was based on literature and on knowledge and experiences of all co-authors working in the fields of hereditary breast and/or ovarian cancer and qualitative research. This guide included questions about influencing factors on the acceptance of both the innovation itself and a study on this new concept. Prior to the interviews, confidentiality was assured and the process of the interview was explained. Written informed consent was gained from all participants. All focus group interviews were introduced by a $5-7$ minute presentation about recent and relevant insights in strategies to reduce the risk of ovarian cancer. The focus group interviews were guided by a chairman (J.H.) and two researchers attended as observers (R.H. and M.A.). The focus group interviews took 90-105 min and were audiotaped after consent of the participants.

\section{Professionals}

Interviews with 23 professionals were performed in seven university hospitals and one nonuniversity hospital (about three professionals per hospital) irrespective of data saturation. In advance we expected different opinions between hospitals, therefore we allowed all professionals to speak about this topic. The interview guide was based on literature and on knowledge and experiences of all co-authors containing the following topics: pros and cons of the new concept (RRS with delayed RRO) for patients, professionals, and organization; bottlenecks of a study on this subject; and suggestions for study design. Prior to the interviews, confidentiality was assured and the process of the interview was explained. The interviews were performed by one researcher (M.A.), took 20-55 min, and were audiotaped after consent.

\section{Analyses}

Both focus group and individual interviews were fully transcribed. Transcripts were independently analyzed by two researchers (M.A. and M.H.). The analyses were conducted with the aid of qualitative analysis tool of ATLAS.ti GmbH Version 6 (Berlin, Germany). We used a process of constant comparison, originating from grounded theory. ${ }^{28}$ At first, we read the complete transcript to become familiar with the data. In a second step, we performed open coding independently of one another, and stayed semantically close to the participants' wording. During this inductive coding process, the four determinants of innovation within health care organizations (at the level of innovation itself, patient, professional, and organization) defined by Fleuren ${ }^{29}$ and $\mathrm{Gro}^{30}$ served as 'sensitizing concepts'. We analyzed the influencing factors for the choice of RRS with delayed RRO instead of RRSO, and subsequently we explored these factors for study participation on this 
concept. Thirdly, we discussed our findings until we achieved mutual agreement. In this way, axial codes were defined. New insights were written down in memos. After constant iteration of these steps, 'selective coding' led to a deep understanding of the barriers and facilitators of RRS with delayed RRO, and of study participation on this subject from both BRCA1/2 mutation carriers' and professionals' perspectives.

As assessed by the institutional review board of the Radboud University Medical Center (CMO no. 2013/050), the study was not subject to the Dutch 'Medical Research Involving Human Subjects Act' meaning that it was exempt from approval.

\section{RESULTS}

\section{BRCA1/2 mutation carriers}

Of 66 BRCA1/2 mutation carriers who were approached, 44 women (67\%) agreed on study participation, of which five eventually were unable to attend. The total participation rate was 59\% (39/66). Characteristics of focus groups are summarized in Table 1. In four focus groups with 39 BRCA1/2 mutation carriers, we identified 18 barriers and facilitators influencing the choice for RRS with delayed RRO. We found two barriers and one facilitator for study participation on this concept. Factors (barriers and facilitators) mentioned in at least two focus groups are listed in Tables 2 and 4. Factors cited in all four focus groups are described in the text and marked in the table with an asterisk $\left(^{*}\right)$. In both tables and text, barriers and facilitators are ranked by the amount of mentioning.

Table 1. Characteristics of focus groups

\begin{tabular}{|c|c|c|c|c|}
\hline & $\begin{array}{c}\text { Focus Group } 1 \\
(N=9)\end{array}$ & $\begin{array}{c}\text { Focus Group } 2 \\
(N=10)\end{array}$ & $\begin{array}{c}\text { Focus Group } 3 \\
(N=11)\end{array}$ & $\begin{array}{c}\text { Focus Group } 4 \\
(N=9)\end{array}$ \\
\hline Duration; minutes & 105 & 105 & 95 & 90 \\
\hline \multicolumn{5}{|l|}{ BRCA mutation; N (\%) } \\
\hline BRCA1 & $8(89)$ & $9(90)$ & $10(91)$ & $5(56)$ \\
\hline$B R C A 2$ & $1(11)$ & $1(10)$ & $1(9)$ & $3(33)$ \\
\hline$B R C A 1+2$ & & & & $1(11)$ \\
\hline Age; median (range) & $46(39-56)$ & $47(42-56)$ & $46(43-55)$ & $37(33-43)$ \\
\hline Age at RRSO; median (range) & $40(37-46)$ & $38.5(35-46)$ & $38(32-41)$ & \\
\hline Years since RRSO; median (range) & $5(2-15)$ & $8(1-16)$ & $11(3-14)$ & \\
\hline History of breast cancer; N (\%) & 0 & $10(100)$ & $5(45)$ & 0 \\
\hline Age at breast cancer; median (range) & & $35(31-48)$ & $38(36-42)$ & \\
\hline RRM; N (\%) & $6(67)$ & & $3(50)$ & $5(56)$ \\
\hline \multicolumn{5}{|l|}{ Preference of strategy; N (\%) } \\
\hline RRSO & & $8(80)$ & $6(55)$ & $3(33)$ \\
\hline RRS with delayed RRO & $7(78)$ & $2(20)$ & $3(27)$ & $5(56)$ \\
\hline Unsure & $2(22)$ & & $2(18)$ & $1(11)$ \\
\hline
\end{tabular}

Abbreviations: RRSO risk-reducing salpingo-oophorectomy; RRM risk-reducing mastectomy; RRS risk-reducing salpingectomy; RRO risk-reducing oophorectomy 
Table 2. Influencing factors for RRS with delayed RRO according to BRCA1/2 mutation carriers

\begin{tabular}{|c|c|c|}
\hline Determinants & Barriers & Facilitators \\
\hline \multirow{3}{*}{$\begin{array}{l}\text { Domain 1: } \\
\qquad \text { Innovation }\end{array}$} & Seriousness of ovarian cancer* & Delay of menopause* \\
\hline & Uncertainty of effect of innovation* & Preserve fertility \\
\hline & Complexity of decision & Earlier preventive option \\
\hline \multirow{5}{*}{$\begin{array}{l}\text { Domain 2: } \\
\text { Patients }\end{array}$} & Family history* & Autonomy* \\
\hline & Ease of decision to RRSO* & Reluctance to premature menopause \\
\hline & Personal history of breast cancer ${ }^{a}$ & \\
\hline & Influence of family/having children & \\
\hline & Difficulty of deciding based on numbers & \\
\hline Domain 3: & Inadequate counselling about effects of RRSO* & \\
\hline Professionals & Limited knowledge on this specialistic subject & \\
\hline Domain 4: & Lack in continuity of professionals & Sufficient information* \\
\hline Organization & & $\begin{array}{l}\text { Approachable contact with } \\
\text { professionals }\end{array}$ \\
\hline
\end{tabular}

Factors mentioned in at least two focus groups are listed and are ranked by the amount of mentioning;

Abbreviations: RRS risk-reducing salpingectomy; RRO risk-reducing oophorectomy; RRSO risk-reducing salpingo-oophorectomy;

*mentioned in all four focus groups;

${ }^{a}$ mentioned in focus groups with participants with a history of breast cancer (focus group 2 and 3 )

\section{Barriers and facilitators related to RRS with delayed RRO}

Domain 1: determinants at innovation level

Two main barriers and one facilitator were mentioned in this domain. Firstly, the seriousness of ovarian cancer was cited as the main barrier. The threat of ovarian cancer was brought up in all focus groups considering its inability of early discovery and its high lethality rate. Another main barrier of the innovation was the uncertainty about the effect of RRS with delayed RRO on the risk of ovarian cancer. According to this barrier, peace of mind after RRSO was cited as an important benefit of RRSO in all four focus groups.

The main facilitator of the innovation for BRCA1/2 mutation carriers was the delay of early menopause considering both physical and psychosocial aspects. From a physical point of view, this innovation could accomplish a delay in several negative effects of RRO, like vasomotor symptoms, sexual dysfunction, joint complaints, acceleration of aging, changes in appearance, and decrease of life expectancy. Positive psychosocial effects were the delay in mood alternations, influence of relationship with partner, sleeping complaints, and energy loss. Furthermore, the delay of menopause could also postpone the difficulties in deciding to use hormone replacement therapy mentioned in all focus groups.

\section{Domain 2: determinants at patient level}

In all focus groups, participants emphasized that family history generally played an important role in decision making for preventive strategies. The main barrier for the innovation was having family members with ovarian cancer. The predominant type of cancer (breast or ovarian) in a family mainly 
affected the anxiety for breast or ovarian cancer. Another main barrier among BRCA1/2 mutation carriers was the ease of decision to undergo RRSO. Different aspects seemed to be connected to this ease, including underestimation of short- and long-term impact of RRSO, limited impact of a laparoscopy, need for certainty/comforting feeling about reducing cancer risk, and furthermore predominant anxiety of cancer and choices based on anticipated regret. Nevertheless, most BRCA1/2 mutation carriers who underwent both RRSO and RRM cited a higher impact of RRSO compared to RRM. Having a personal history of breast cancer was identified as the main barrier in both focus groups with participants with prior breast cancer. The restraint in study participation on this innovation was mainly based on the need for certainty and to exclude all possible risks developing cancer again.

Need for maintenance of autonomy was identified as the main facilitator. BRCA1/2 mutation carriers in our focus groups attached an important value to freedom of choice, and the significance of individual considerations was underlined.

\section{Domain 3: determinants at professional level}

BRCA1/2 mutation carriers reported insufficient counseling about effects of RRSO as the main barrier, and seemed to be in close connection with the ease of decision to undergo RRSO.

\section{Domain 4: determinants at organization level}

In general, sufficient information was the main facilitator for making decisions in this group of BRCA1/2 mutation carriers. In all focus groups, different needs for information were mentioned consisting of new medical insights, contact with peers, group information, and/or contact with professionals. Specially, detailed explanation of the term 'ovarian cancer' and its origin was mentioned to be valuable, referring to the expected effect of RRS.

\section{Barriers related to study participation}

In all focus groups, acceptable designs to study the added value of RRS with delayed RRO instead of RRSO were discussed. The main discussion point was the concept of randomization and its preference as ideal study design. In all focus groups, a general consensus of opinion was that a randomized study design generates a main barrier for participation in a future study. The most important reasons for this aversion were the need for freedom of choice and maintenance of autonomy.

All focus group interviews finished with questions about the hypothetic choice between RRSO and RRS with delayed RRO (Table 1). In summary, 17 women were interested in the new strategy (44\%), 17 women preferred the current strategy (44\%), and five were unsure (12\%). 


\section{Professionals}

Of all 23 professionals, four were males (one gynecological oncologist, one clinical geneticist and two surgeons taking care of breast surveillance). Factors mentioned by at least two professionals are listed in Tables 3 and 4. In total, 20 barriers and facilitators influencing the choice for RRS with delayed RRO were identified (Table 3). For study participation, 10 barriers and facilitators were denominated at the level of patients, professionals and organization (Table 4). Factors mentioned by more than $50 \%$ of the professionals are described in the text and marked in the table with asterisks $\left.{ }^{(* *}\right)$. In both tables and text, barriers and facilitators are ranked by the amount of mentioning.

Table 3. Influencing factors for RRS with delayed RRO according to professionals

\begin{tabular}{|c|c|c|}
\hline Determinants & Barriers & Facilitators \\
\hline $\begin{array}{l}\text { Domain 1: } \\
\text { Innovation }\end{array}$ & $\begin{array}{l}\text { Undergoing two operations* } \\
\text { Delay of risk reduction of breast cancer* } \\
\text { Risks attended by two operations* } \\
\text { Uncertainty of effect of innovation* } \\
\text { Seriousness of ovarian cancer }\end{array}$ & $\begin{array}{l}\text { Delay of menopause* } \\
\text { Preserve fertility } \\
\text { Delay of HRT use } \\
\text { Earlier examination of tubes }\end{array}$ \\
\hline $\begin{array}{l}\text { Domain 2: } \\
\text { Patients }\end{array}$ & $\begin{array}{l}\text { Ease of decision to RRSO* } \\
\text { Family history }\end{array}$ & $\begin{array}{l}\text { Reluctance to premature menopause } \\
\text { Well-informed and involved patients }\end{array}$ \\
\hline $\begin{array}{l}\text { Domain 3: } \\
\quad \text { Professionals }\end{array}$ & $\begin{array}{l}\text { Insufficient evidence* } \\
\text { Insufficient knowledge of professionals }\end{array}$ & General scientific attitude \\
\hline $\begin{array}{l}\text { Domain 4: } \\
\text { Organization }\end{array}$ & $\begin{array}{l}\text { Increase in health care consumption } \\
\text { Cooperation between hospitals } \\
\text { Lack of capacity }\end{array}$ & $\begin{array}{l}\text { Cooperation in multidisciplinary teams on } \\
\text { hereditary cancer }\end{array}$ \\
\hline
\end{tabular}

Factors mentioned by at least two professionals are listed in this table and are ranked by the amount of mentioning; RRS risk-reducing salpingectomy; RRO risk-reducing oophorectomy; RRSO risk-reducing salpingooophorectomy;

${ }^{*}$ mentioned by more than $50 \%$ of the professionals

\section{Barriers and facilitators related to RRS with delayed RRO}

Domain 1: determinants at innovation level

Four main barriers were identified in this domain. Undergoing two operations were mentioned as a barrier by almost all professionals. Furthermore, inherent risks of two operations were named as a barrier, including the entailed doubled peri-operative risk, the possibly increased complication risk of RRO after earlier RRS, and the risk of coexisting co-morbidity. The majority of professionals nominated the delay of risk reduction of breast cancer owing to the delay of RRO as a barrier with the comment that an RRM would nullify this barrier. For professionals in breast surveillance this barrier seemed less important. The last important barrier was the uncertainty of the effect of RRS with delayed RRO. To overcome this barrier, some professionals suggested to perform more research on the origin of ovarian cancer, while the majority of professionals thought that a clinical study on this new strategy is most appropriate. 
The main facilitator of this new strategy was the delay of menopause. The majority of professionals mentioned that delay of vasomotor and sexual complaints, osteoporosis, and cardiovascular disease together with maintenance of quality of life are main advantages of the delay of menopause.

Table 4. Influencing factors for study participation according to BRCA1/2 mutation carriers and professionals

\begin{tabular}{|c|c|c|}
\hline & Barriers & Facilitators \\
\hline \multicolumn{3}{|c|}{$B R C A 1 / 2$ mutation carriers ${ }^{a}$} \\
\hline $\begin{array}{l}\text { Domain 2: } \\
\text { Patients }\end{array}$ & & Value of participation for next generation \\
\hline $\begin{array}{l}\text { Domain 4: } \\
\text { Organization }\end{array}$ & $\begin{array}{l}\text { Randomized study design* } \\
\text { Cost of study / insufficient cover by insurance }\end{array}$ & \\
\hline \multicolumn{3}{|c|}{ Professionals $^{b}$} \\
\hline $\begin{array}{l}\text { Domain 2: } \\
\text { Patients }\end{array}$ & Amount of other undoing studies & $\begin{array}{l}\text { Willingness to participate in study** } \\
\text { Value of participation for next generation }\end{array}$ \\
\hline $\begin{array}{l}\text { Domain 3: } \\
\quad \text { Professionals }\end{array}$ & $\begin{array}{l}\text { Time investment } \\
\text { Diversity of viewpoints of professionals }\end{array}$ & Willingness to participate in studies \\
\hline $\begin{array}{l}\text { Domain 4: } \\
\text { Organization }\end{array}$ & $\begin{array}{l}\text { Insufficient cover by insurance } \\
\text { Differences in pathology examination of tubes } \\
\text { Assessment by institutional review board }\end{array}$ & Uniform counseling** \\
\hline
\end{tabular}

a Factors mentioned in at least two focus groups are listed;

${ }^{b}$ Factors mentioned by at least two professionals are listed;

*mentioned in all focus groups;

**mentioned by more than $50 \%$ of the professionals

\section{Domain 2: determinants at BRCA1/2 mutation carrier level}

The ease of the decision of BRCA1/2 mutation carriers to undergo RRSO was mentioned as the main barrier. According to professionals, BRCA1/2 mutation carriers generally underestimate the impact of early menopause, receive limited information concerning side effects of RRSO, and are less emotionally attached to their ovaries compared to breasts and uterus.

\section{Domain 3: determinants at professional level}

In this domain, the main barrier for professionals was insufficient scientific foundations of this new strategy. The major drawback was the unproven effect of RRS as a preventive strategy, and the unknown effect of delaying $\mathrm{RRO}$ on the risk of ovarian cancer.

\section{Domain 4: determinants at organization level}

No barriers or facilitators were mentioned by more than $50 \%$ of the professionals; therefore, no main factors of influence at the level of organization were expected.

\section{Barriers and facilitators related to study participation}

The main facilitator at patient level mentioned by almost all professionals was the expected willingness of a substantial part of BRCA1/2 mutation carriers to participate in a study on RRS with delayed RRO. 
At organization level, the main facilitator for study participation was uniform counseling according to a widely supported study protocol. Important remarks about study design were the need for an observational study with long-term follow-up concentrating on ovarian cancer risk and quality of life. Furthermore, professionals mentioned the need for a reliable power calculation, and clear, extensive, and scientifically based patient information considering all pros and cons of this new strategy.

\section{DIScussion}

Our study identified a wide variety of barriers and facilitators influencing the choice for RRS with delayed RRO instead of RRSO in BRCA1/2 mutation carriers and their treating professionals. Barriers identified on the level of the innovation itself and on the level of BRCA1/2 mutation carriers appeared to provide the most important impact. Both BRCA1/2 mutation carriers and professionals experienced the uncertainty of the effect of the innovation, and the ease of the decision to undergo RRSO as main barriers. Among BRCA1/2 mutation carriers, the other main barriers were 1) seriousness of ovarian cancer; 2) family history; 3) personal history of breast cancer; and 4) inadequate information about all side effects of RRSO. Among professionals, undergoing two operations with its inherent additional risks, delay of risk reduction of breast cancer, and insufficient supportive evidence were recognized as main barriers. Delay of menopause was suggested as the main facilitator for both BRCA1/2 mutation carriers and professionals. BRCA1/2 mutation carriers mentioned autonomy in the choice between RRSO and RRS with delayed RRO, and sufficient information as main facilitators.

For study participation, additional barriers and facilitators were identified. A randomized study design was mentioned to be the main barrier for BRCA1/2 mutation carriers. Among professionals, main facilitators were expected willingness of BRCA1/2 mutation carriers to participate and uniform counseling. Overall, a general interest in a non-randomized observational study of RRS with delayed RRO was observed taking into account all mentioned barriers and facilitators. Apparently, a substantial part of the BRCA1/2 mutation carriers (43.6\%) indicated interest in RRS with delayed $\mathrm{RRO}$, which is slightly higher than the $34.3 \%$ reported by Holman et al. ${ }^{27}$ Possible explanations for this difference could be the size of the study groups ( $n=39$ versus $n=204$ ), type of research (qualitative versus quantitative), and included BRCA1/2 mutation carriers (before and/or after risk reducing strategies). Almost all professionals in our study were also interested to participate in a study on this innovation.

Elaborating on this innovation as possible addition to the current strategy, suggestions are made by Anderson et al. to perform a clinical trial to test the equivalence of RRS to RRSO in reducing pelvic serous cancer risk in BRCA mutation carriers, needing up to 4000 women in each $\mathrm{arm} .{ }^{25} \mathrm{~A}$ recent decision-analytic model of Kwon ${ }^{24}$ compared costs, risks, and benefits of three strategies (RRS alone, 
RRS with delayed RRO, and RRSO) confirming the greatest risk reduction for breast and pelvic serous cancer of the current standard RRSO. However, when considering quality-adjusted life expectancy, RRS with delayed RRO is a cost-effective strategy. ${ }^{24}$ The evidence regarding RRS with delayed RRO is still limited, and only two clinical trials on this subject are currently recruiting BRCA1/2 mutation carriers. A French study by Leblanc and colleagues is currently offering salpingectomy to BRCA1/2 mutation carriers who are reluctant to undergo RRSO (NCT01608074). In addition, an American research group recently started a study on patient compliance with the strategy consisting of RRS with delayed RRO (NCT01907789). Altogether, there is an increasing interest in the role of RRS with delayed RRO for BRCA1/2 mutation carriers. Findings of our study will undoubtedly help to foresee difficulties in designing and implementing new studies on this subject. Based on our study, we designed a multicenter prospective non-randomized controlled study with menopause-related quality of life as primary outcome measure. BRCA1/2 mutation carriers will choose between the current standard (RRSO) and RRS with delayed RRO (NCT02321228).

Appreciable differences in main barriers were found between BRCA1/2 mutation carriers and professionals at the level of innovation. The first difference was the interest in influence of the delay of breast cancer reduction as a result of the new strategy. Professionals reported this as the main barrier, whereas this delay was not a relevant issue for BRCA1/2 mutation carriers considering better available strategies for breast cancer reduction, irrespectively of previous RRM. Only some BRCA1/2 mutation carriers mentioned the breast cancer risk reduction of RRSO as an additional advantage; however, the delay of risk reduction due to the new strategy is a matter of minor significance. From patients' perspective, the suggestion of Anderson et al..$^{25}$ to perform a clinical trial in BRCA1/2 mutation carriers who previously underwent RRM is therefore questionable. Unfortunately, Holman et al. ${ }^{27}$ did not ask their participants of their thoughts about the delay of reduction of breast cancer. The altering insight of the scarcely reduction of breast cancer risk by RRSO at premenopausal age ${ }^{8,9}$ might give an even more important role for RRM in young BRCA1/2 mutation carriers. The second difference between BRCA1/2 mutation carriers and professionals was the judgment on the inevitability of two operations and its additional risks involved with the new strategy. This was considered as the main barrier by professionals, whereas BRCA1/2 mutation carriers explicitly attached little importance to this aspect.

Another consideration for participation in a study of RRS with delayed RRO was the possible influence of a personal history of breast cancer. Only two of the 15 women with a history of breast cancer (13\%) were interested in the option of RRS with delayed RRO. It may be assumable that a personal history of cancer makes women more willing to minimize their future cancer risk and to accept the negative effects of RRO. However, earlier studies on uptake of RRSO showed conflicting results on the predictive value of a personal history of breast cancer. ${ }^{31-33}$

Potential ovarian damage was suggested by Holman et al. ${ }^{27}$ as a reason for not participating in a study on RRS with delayed RRO. Recently, no negative effects on the ovarian function were found in premenopausal low-risk women for ovarian cancer who underwent bilateral salpingectomy in 
addition to laparoscopic hysterectomy for benign uterine pathologies. ${ }^{34}$ In our study, the potential effects of RRS on ovarian function were not mentioned as a barrier by BRCA1/2 mutation carriers or professionals.

Our study is the first reported performance of an in-depth qualitative analysis of the new concept of RRS with delayed RRO from both patients' and professionals' perspectives. Another strength is the considerable number of participating BRCA1/2 mutation carriers, and the different constitutions of focus groups regarding medical background. Furthermore, professionals from almost all Dutch university hospitals participated in our study representing three different disciplines.

A possible limitation of our study is that all focus groups with only Caucasian BRCA1/2 mutation carriers were performed in one hospital. Performing only one focus group with BRCA1/2 mutation carriers prior to RRSO might have caused a biased view. Although, we thought that BRCA1/2 mutation carriers after RRSO could have provided an important contribution to amplify the impact of RRSO and to explain the value of a history of breast cancer on the subject of our study. Furthermore, BRCA1 mutation carriers were overrepresented in our study. This might be partly explained by a higher invitation rate (46 BRCA1 and 20 BRCA2 mutation carriers, 70\% vs. 30\%), although there was also an unforeseen higher participation rate (33 BRCA1 and 6 BRCA2 mutation carriers; $72 \%$ vs. $30 \%$ ). This might be due to coincidence; however, it is also possible that the subject concerned BRCA1 mutation carriers more because of the higher risk and the earlier age of onset of ovarian cancer. The feasibility of internationalization of our results may be difficult to appraise, because of unknown or unforeseen international difference, for example insurance systems. Although, the interest in study participation on this innovation is in accordance with the results of Holman, ${ }^{27}$ both studies elucidate various aspects of RRS with delayed RRO.

In summary, our study provides new and additional data on influencing factors of BRCA1/2 mutation carriers and their treating professionals for RRS with delayed RRO as a substitute for RRSO and for study participation on this concept. Knowledge of these factors may help to improve the design and implementation of a non-randomized study on this subject in which special attention should be paid to scientific basis, importance of autonomy, and providing sufficient information.

\section{Acknowledgments}

This work was supported by a Ruby and Rose grant. We would like to express our appreciation to the participating BRCA1/2 mutation carriers and the participating professionals from the Dutch hospitals, namely the Center for Gynaecological Oncology Amsterdam, Erasmus University MCDaniel den Hoed Cancer Center, Leiden University Medical Center, Maastricht University Medical Center, NKI-AVL, Tweesteden Hospital Tilburg, University Medical Center Groningen, and University Medical Center Utrecht. 


\section{REFEREMCES}

1. Alsop K, Fereday S, Meldrum C, et al. BRCA mutation frequency and patterns of treatment response in BRCA mutation-positive women with ovarian cancer: a report from the Australian Ovarian Cancer Study Group. J Clin Oncol 2012; 30(21): 2654-63.

2. Zhang S, Royer R, Li S, et al. Frequencies of BRCA1 and BRCA2 mutations among 1,342 unselected patients with invasive ovarian cancer. Gynecol Oncol 2011; 121(2): 353-7.

3. Risch HA, McLaughlin JR, Cole DE, et al. Prevalence and penetrance of germline BRCA1 and BRCA2 mutations in a population series of 649 women with ovarian cancer. Am J Hum Genet 2001; 68(3): 70010.

4. Chen S, Parmigiani G. Meta-analysis of BRCA1 and BRCA2 penetrance. J Clin Oncol 2007; 25(11): 132933.

5. Brohet RM, Velthuizen ME, Hogervorst FB, et al. Breast and ovarian cancer risks in a large series of clinically ascertained families with a high proportion of BRCA1 and BRCA2 Dutch founder mutations. J Med Genet 2014; 51(2): 98-107.

6. Rebbeck TR, Kauff ND, Domchek SM. Meta-analysis of risk reduction estimates associated with riskreducing salpingo-oophorectomy in BRCA1 or BRCA2 mutation carriers. J Nat/ Cancer Inst 2009; 101(2): 80-7.

7. Eisen A, Lubinski J, Klijn J, et al. Breast cancer risk following bilateral oophorectomy in BRCA1 and BRCA2 mutation carriers: an international case-control study. J Clin Oncol 2005; 23(30): 7491-6.

8. Mavaddat N, Peock S, Frost D, et al. Cancer risks for BRCA1 and BRCA2 mutation carriers: results from prospective analysis of EMBRACE. J Natl Cancer Inst 2013; 105(11): 812-22.

9. Fakkert IE, Mourits MJ, Jansen L, et al. Breast cancer incidence after risk-reducing sal pingo-oophorectomy in BRCA1 and BRCA2 mutation carriers. Cancer Prev Res 2012; 5(11): 1291-7.

10. Finch A, Metcalfe KA, Chiang JK, et al. The impact of prophylactic salpingo-oophorectomy onmenopausal symptoms and sexual function in women who carry a BRCA mutation. Gynecol Oncol 2011; 121(1): $163-8$.

11. Madalinska JB, van Beurden M, Bleiker EM, et al. The impact of hormone replacement therapy on menopausal symptoms in younger high-risk women after prophylactic salpingo-oophorectomy. J Clin Oncol 2006; 24(22): 3576-82.

12. Atsma F, Bartelink ML, Grobbee DE, van der Schouw YT. Postmenopausal status and early menopause as independent risk factors for cardiovascular disease: a metaanalysis. Menopause (NY) 2006; 13(2): 265-79.

13. Svejme O, Ahlborg HG, Nilsson JA, Karlsson MK. Early menopause and risk of osteoporosis, fracture and mortality: a 34-year prospective observational study in 390 women. BJOG 2012; 119(7): 810-6.

14. Rocca WA, Grossardt BR, Maraganore DM. The long-term effects of oophorectomy on cognitive and motor aging are age dependent. Neurodegener Dis 2008; 5(3-4): 257-60.

15. Bolton KL, Chenevix-Trench G, Goh C, et al. Association between BRCA1 and BRCA2 mutations and survival in women with invasive epithelial ovarian cancer. JAMA 2012; 307(4): 382-90.

16. Kurman RJ, Shih le M. The origin and pathogenesis of epithelial ovarian cancer: a proposed unifying theory. Am J Surg Pathol 2010; 34(3): 433-43.

17. Crum CP, Drapkin R, Miron A, et al. The distal fallopian tube: a new model for pelvic serous carcinogenesis. Curr Opin Obstet Gynecol 2007; 19(1): 3-9.

18. Roh MH, Kindelberger D, Crum CP. Serous tubal intraepithelial carcinoma and the dominant ovarian mass: clues to serous tumor origin? Am J Surg Pathol 2009; 33(3): 376-83.

19. Kindelberger DW, Lee $Y$, Miron A, et al. Intraepithelial carcinoma of the fimbria and pelvic serous carcinoma: evidence for a causal relationship. Am J Surg Pathol 2007; 31(2): 161-9.

20. Roh MH, Yassin Y, Miron A, et al. High-grade fimbrial-ovarian carcinomas are unified by altered p53, PTEN and PAX2 expression. Mod Pathol Off J U S Can Acad Pathol Inc 2010; 23(10): 1316-24. 
21. Folkins AK, Jarboe EA, Saleemuddin A, et al. A candidate precursor to pelvic serous cancer (p53 signature) and its prevalence in ovaries and fallopian tubes fromwomen with BRCA mutations. Gynecol Oncol 2008; 109(2): 168-73.

22. Mingels MJ, Roelofsen T, van der Laak JA, et al. Tubal epithelial lesions in salpingo-oophorectomy specimens of BRCA-mutation carriers and controls. Gynecol Oncol 2012; 127(1): 88-93.

23. Dubeau L, Drapkin R. Coming into focus: the nonovarian origins of ovarian cancer. Ann Oncol 2013; 24(Suppl. 8):viii28-35.

24. Kwon JS, Tinker A, Pansegrau G, et al. Prophylactic salpingectomy and delayed oophorectomy as an alternative for BRCA mutation carriers. Obstet Gynecol 2013; 121(1): 14-24.

25. Anderson CK, Wallace S, Guiahi M, Sheeder J, Behbakht K, Spillman MA. Riskreducing salpingectomy as preventative strategy for pelvic serous cancer. Int J Gynecol Cancer 2013; 23(3): 417-21.

26. Leblanc E, Narducci F, Farre I, et al. Radical fimbriectomy: a reasonable temporary risk-reducing surgery for selected women with a germ line mutation of BRCA 1 or 2 genes? Rationale and preliminary development. Gynecol Oncol 2011;121(3):472-6.

27. Holman LL, Friedman S, Daniels MS, Sun CC, Lu KH. Acceptability of prophylactic salpingectomy with delayed oophorectomy as risk-reducing surgery among BRCA mutation carriers. Gynecol Oncol 2014; 133(2): 283-6.

28. Mortelmans D. Handboek kwalitatieve onderzoeksmethoden. 1e dr. In: Leuven, Voorburg, Acco, editors. 2007 [p. 534].

29. Fleuren M, Wiefferink K, Paulussen T. Determinants of innovation within health care organizations: literature review and Delphi study. Int J Qual Health Care J Int Soc Qual Health Care/ISQua 2004; 16(2):10723.

30. Grol R, Wensing M, Eccles M. Improving patient care: the implementation of change in clinical practice. New York: Edinburgh, Elsevier Butterworth Heinemann; 2005.

31. Beattie MS, Crawford B, Lin F, Vittinghoff E, Ziegler J. Uptake, time course, and predictors of risk-reducing surgeries in BRCA carriers. Genet Test Mol Biomarkers 2009; 13(1): 51-6.

32. Manchanda R, Burnell $M$, Abdelraheim A, et al. Factors influencing uptake and timing of risk reducing salpingo-oophorectomy in women at risk of familial ovarian cancer: a competing risk time to event analysis. BJOG 2012; 119(5): 527-36.

33. Sidon L, Ingham S, Clancy T, et al. Uptake of riskreducing salpingo-oophorectomy in women carrying a BRCA1 or BRCA2 mutation: evidence for lower uptake in women affected by breast cancer and older women. Br J Cancer 2012; 106(4):775-9.

34. Morelli M, Venturella R, Mocciaro R, et al. Prophylactic salpingectomy in premenopausal low-risk women for ovarian cancer: primum non nocere. Gynecol Oncol 2013; 129(3): 448-51. 



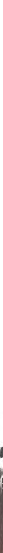

\section{Salpingectomy with}

delayed oophorectomy in BRCA1/2

mutation camriens:

estimating ovariaw cancer risk

Marline G. Harmsen, Joanna in 't Hout, Marieke Arts-de Jong, Nicoline Hoogerbrugge, Leon F.A.G. Massuger, Rosella P.M.G. Hermens, Joanne A de Hullu 


\section{ABSTRACT}

\section{Objective}

To estimate BRCA1/2 mutation carriers' cumulative ovarian cancer risks after risk-reducing salpingectomy at various ages with delayed oophorectomy several years later compared with riskreducing salpingo-oophorectomy.

\section{Methods}

A literature search was performed on cumulative ovarian cancer risks and effects of risk-reducing salpingo-oophorectomy and salpingectomy. Results were used in a modeling study to estimate cumulative ovarian cancer risks for various scenarios of sal pingectomy with delayed oophorectomy and risk-reducing salpingo-oophorectomy using Cox proportional hazard models.

\section{Results}

Estimated cumulative ovarian cancer risks at age 70 years for risk-reducing salpingectomy with delayed oophorectomy are highest for BRCA1 mutation carriers undergoing surgeries at higher age. Maximum increase in point estimates (from 1.8\% to 4.1\%) occurs in 40-year-old BRCA1 mutation carriers undergoing oophorectomy at age 45 years after nonprotective salpingectomy instead of salpingo-oophorectomy at age 40 years. In the best-case scenario, assuming 65\% risk reduction by salpingectomy and $96 \%$ by salpingo-oophorectomy, point estimates increase (from $1.8 \%$ to 2.6\%) or decrease (from 3.4\% to 3.3\%) depending on age. In the worst-case scenario for BRCA2, point estimates maximally increase from $0.6 \%$ to $1.8 \%$ in 45 -year-old carriers when oophorectomy is performed at age 50 years instead of risk-reducing salpingo-oophorectomy at age 45 years. In the best-case scenario, point estimates increase (from 1.3\% to 1.5\%) or decrease (from 1.5 to 1.3\%).

\section{Conclusion}

Differences in estimated ovarian cancer risks between risk-reducing salpingo-oophorectomy and salpingectomy with delayed oophorectomy are small, even if salpingectomy is ineffective. Presented estimated ovarian cancer risks can be used in counseling BRCA1/2 mutation carriers, thereby facilitating a personalized and well-informed choice for either strategy. 


\section{InTRODUCTION}

Germline mutations in the BRCA1/2 genes are associated with high susceptibility for breast and ovarian cancer and these mutations are highly penetrant.' Risk-reducing salpingo-oophorectomy is the only proven surgical strategy in reducing ovarian cancer risk., Until recently, risk-reducing salpingo-oophorectomy was also thought to reduce breast cancer risk by half when performed before natural menopause; ${ }^{2}$ however, this is contradicted by several recent publications. ${ }^{4-6}$

The most important disadvantage of salpingo-oophorectomy at premenopausal age is onset of premature menopause, which is known to have several short- and long-term adverse effects like vasomotor symptoms, sexual dysfunction, osteoporosis, cardiovascular disease, cognitive impairment, and increased risk of premature death. ${ }^{7-10}$ Hormone therapy alleviates most menopausal effects to greater or lesser extent but not all.? Therefore, a risk-reducing strategy enabling preservation of the ovaries and thereby increasing quality of life may be of great advantage. Together with the growing evidence for the fallopian tube as the place of origin of ovarian carcinoma, ${ }^{11-14}$ an alternative risk-reducing strategy has been suggested. ${ }^{15-20}$ This consists of early risk-reducing salpingectomy upon completion of childbearing followed by delayed riskreducing oophorectomy several years later than currently recommended. However, data on its effects on ovarian cancer risk are lacking. We need optimal insight into ovarian cancer risks before offering this alternative risk-reducing strategy to BRCA mutation carriers. Therefore, the aim of this study is to estimate cumulative ovarian cancer risks for this alternative strategy and to compare them with risks under standard risk-reducing salpingo-oophorectomy with the ultimate goal to personalize and optimize risk communication.

\section{MATERIALS AMD METHODS}

\section{Literature search}

We determined three important variables in estimating cumulative ovarian cancer risks, which were included in this modeling study: 1) cumulative ovarian cancer risks for BRCA1/2 mutation carriers, 2) the ovarian cancer risk reduction attributable to risk-reducing salpingo-oophorectomy, and 3) the assumed effect of risk-reducing sal pingectomy based on the distribution of the histotypes of BRCArelated ovarian carcinomas. An extensive review of the literature was performed on these variables to obtain hazard ratios as input for our risk model (Table 1). The MEDLINE database was searched for publications between 1964 and September 1, 2014 (date of our last search) using the following terms: 1) MeSH: Penetrance; Genes, BRCA1; Genes, BRCA2. Title\&abstract: Meta-analysis; 2) title and abstract: Salpingo-oophorectomy; Salpingectomy; Oophorectomy; BRCA1; BRCA2; BRCA; Metaanalysis; 3) MeSH: Humans; Title\&Abstract: BRCA1; BRCA2; BRCA; Ovarian Cancer; Ovarian Carcinoma; Mutation Carrier(s); Germline; Pathology; Histology; Morphology; Epithelial. Titles and abstracts of 
the identified 3, 10, and 171 reports, respectively, were read by one of the authors (M.G.H.) to select articles that were likely to report the requested effect sizes. Full texts of the remaining articles were scrutinized by one of the authors (M.G.H.) to further determine relevance and applicable hazard ratios were extracted from the relevant articles describing the largest patient groups as input for our model: one, two, and three articles, respectively. When available, most recent meta-analyses were considered best evidence (level I). The entire selection procedure was separately checked by one of the authors (J.A.d.H.). Whenever broad ranges in published data existed, we ran the model for the "best-case" and "worst-case" scenarios.

Table 1. Overview of the literature search

\begin{tabular}{|c|c|c|c|c|}
\hline Parameter & MEDLINE Search & Hits & $\begin{array}{l}\text { Screening title } \\
\text { and abstract on } \\
\text { relevance }\end{array}$ & $\begin{array}{l}\text { Selected after } \\
\text { reading full } \\
\text { text }\end{array}$ \\
\hline $\begin{array}{l}\text { Cumulative ovarian } \\
\text { cancer risks }\end{array}$ & $\begin{array}{l}\text { MeSH Terms: Penetrance; Genes, BRCA1; } \\
\text { Genes, BRCA2 } \\
\text { Title\&Abstract: Meta-analysis }\end{array}$ & 3 & 2 & Chen $2007^{1}$ \\
\hline $\begin{array}{l}\text { Efficacy of RRSO in } \\
\text { reduction of ovarian } \\
\text { carcinoma }\end{array}$ & $\begin{array}{l}\text { MeSH Terms: - } \\
\text { Title\&Abstract: Sal pingo-oophorectomy; } \\
\text { Salpingectomy; Oophorectomy; BRCA1; } \\
\text { BRCA2; BRCA; Meta-analysis }\end{array}$ & 10 & 2 & $\begin{array}{l}\text { Marchetti } 2014^{3} \\
\text { Rebbeck } 2009^{2}\end{array}$ \\
\hline $\begin{array}{l}\text { Assumptions on } \\
\text { efficacy of RRS } \\
\text { (percentage of serous } \\
\text { carcinomas among } \\
\text { BRCA-related ovarian } \\
\text { carcinomas) }\end{array}$ & $\begin{array}{l}\text { MeSH Term: Humans. } \\
\text { Title\&Abstract: BRCAT; BRCA2; BRCA; } \\
\text { Ovarian Cancer; Ovarian Carcinoma; } \\
\text { Mutation Carrier(s); Germline; Pathology; } \\
\text { Histology; Morphology; Epithelial }\end{array}$ & 171 & 7 & $\begin{array}{l}\text { Russo } 2009^{8} \\
\text { Bolton } 2012^{24} \\
\text { Mavaddat } 2012^{25}\end{array}$ \\
\hline
\end{tabular}

Abbreviations: RRSO, risk-reducing salpingo-oophorectomy; RRS, risk-reducing salpingectomy.

\section{Data and assumptions}

Chen et al. ${ }^{1}$ performed a meta-analysis of 10 studies to calculate mean cumulative ovarian cancer risks for $B R C A 1 / 2$ mutation carriers until the age of 70 years taking into account competing deaths (Table 2). In our opinion, this most recent meta-analysis reflects the best available evidence (i.e., level I) and is worldwide frequently referred to when cumulative risks in BRCA1/2 mutation carriers are described.

Table 2. Mean cumulative risks for ovarian cancer at a certain age for a 20-year-old mutation carrier in absence of any risk-reducing surgery

\begin{tabular}{lccccc}
\hline & \multicolumn{5}{c}{ Age (y) } \\
\cline { 2 - 6 } Gene & $\mathbf{3 0}$ & $\mathbf{4 0}$ & $\mathbf{5 0}$ & $\mathbf{6 0}$ & $\mathbf{7 0}$ \\
\hline$B R C A 1$ & $1(0.68-1.8)$ & $3.2(2.3-5.1)$ & $9.5(7.3-13)$ & $23(18-28)$ & $39(34-44)$ \\
$B R C A 2$ & $0.19(0.09-.47)$ & $0.7(0.37-1.5)$ & $2.6(1.5-4.5)$ & $7.5(5.1-11)$ & $16(12-20)$ \\
\hline
\end{tabular}

Data are \% (95\% confidence interval).

Data from Chen S, Parmigiani G. Meta-analysis of BRCA1 and BRCA2 penetrance. J Clin Oncol 2007;25:1329-33. 
Risk-reducing salpingo-oophorectomy is well established in reducing ovarian cancer risk in BRCA1/2 mutation carriers. Although various hazard ratios (HRs) were found in previous studies (HR 0.040.29), two meta-analyses were quite consistent and showed HRs of 0.21 and 0.19 corresponding to a risk reduction of approximately $80 \%$., $^{2,3}$

Rebbeck et al. ${ }^{2}$ performed a fixed-effects meta-analysis of pooled results from three nonoverlapping publications. Among 2,840 BRCA1/2 mutation carriers, an HR of 0.21 (95\% confidence interval (CI) $0.12-0.39)$ for ovarian cancer was found for risk-reducing salpingo-oophorectomy. A second fixedeffects meta-analysis by Marchetti et al. ${ }^{3}$ included three studies, one overlapping Rebbeck et al. ${ }^{2}$ and two more recent prospective cohort studies. A total number of 9,192 BRCA1/2 mutation carriers were included; $46 \%$ had undergone risk-reducing salpingo-oophorectomy. They found an HR of 0.19 (95\% Cl 0.13-0.27) for ovarian cancer after risk-reducing salpingo-oophorectomy. ${ }^{3}$ Sufficient data are lacking to discriminate between BRCA1 and BRCA2 mutation carriers with respect to the effect of risk-reducing salpingo-oophorectomy. In conclusion, the reported HRs for the whole group of BRCA1/2 mutation carriers are close to 0.20 , so we assumed an HR of 0.20 for risk-reducing salpingo-oophorectomy (i.e., $80 \%$ risk reduction).

However, the HR of 0.20 might be an underestimation of the effect of risk-reducing salpingooophorectomy. First, the mean age at salpingo-oophorectomy was roughly 45 years in the previously mentioned studies, whereas current guidelines recommend risk-reducing salpingooophorectomy at age 35-40 (BRCA1) or 40-45 (BRCA2). Second, it was not always clear whether complete salpingo-oophorectomy or oophorectomy alone was performed, the latter performed at times until the role of the fallopian tube became illuminated. Furthermore, histologic assessment of earlier salpingo-oophorectomy specimens might have missed occult carcinomas, because the protocol for sectioning and extensively examining the fimbriated end of the fallopian tube was introduced in 2006.21,22 Follow-up of patients was continued until after the introduction of sectioning and extensively examining the fimbriated end of the fallopian tube in only two of eight studies in the meta-analyses. When metastasized disease was subsequently misdiagnosed as primary peritoneal cancer, risk reduction of salpingo-oophorectomy could be underestimated. In conclusion, inclusion of older patients who might have undergone oophorectomy only or whose fallopian tubes were not fully examined in aforementioned studies could have led to underestimation of the effect. Therefore, we decided to also run the model with the best effect of risk-reducing salpingo-oophorectomy ever reported: HR of 0.04 (95\% Cl 0.01-0.16), corresponding to $96 \%$ risk reduction. ${ }^{23}$

No published data on the risk-reducing effect of salpingectomy in BRCA1/2 mutation carriers are available. The theory of ovarian carcinoma arising from the fallopian tube is related to the serous histotype. Therefore, we extracted data on the proportion of the serous histotype within BRCArelated ovarian carcinoma to estimate the possible risk-reducing effect of salpingectomy. Two reviews ${ }^{8,24}$ and one original study using a large international database ${ }^{25}$ report on this. Altogether, approximately $65 \%$ of BRCA-related ovarian carcinomas are of the serous histotype (Table 3 ), 
which equals the distribution within sporadic ovarian carcinoma. ${ }^{24}$ Assuming that most serous cancers originate from the fallopian tube, we expect that the best possible HR for risk-reducing salpingectomy lies around 0.35 , that is, $65 \%$ risk reduction.

However, the effect of risk-reducing salpingectomy can turn out to be greater because specimens in these large studies were not necessarily assessed or revised by a specialized gynecologic pathologist. Revision has been shown to partially reclassify former endometrioid and clear cell carcinomas as serous carcinomas, thereby slightly increasing the amount of serous carcinomas and indirectly the potential risk-reducing effect of salpingectomy. ${ }^{26}$ Furthermore, links between the fallopian tube and endometrioid and mucinous ovarian carcinomas have been described as well: through retrograde menstruation and endometriosis for the first and through Walthard cell nests, paratubal cysts and Brenner tumors for the latter. ${ }^{14}$ The effect of risk-reducing salpingectomy thus could be greater than we now assume if it is not limited to the serous histotype. In contrast, there is still a possibility that salpingectomy does not reduce ovarian cancer risk at all and it is uncertain at what age it should be performed. Therefore, we ran scenarios with HRs of either 0.35 or 1.0 for risk-reducing salpingectomy.

Table 3. Distribution of the serous histotype within BRCA-related ovarian carcinoma

\begin{tabular}{lcccc}
\hline \multirow{2}{*}{ Study } & \multicolumn{2}{c}{ BRCA1 } & \multicolumn{1}{c}{ BRCA2 } \\
\cline { 2 - 4 } & $\mathbf{n}$ & Serous $\boldsymbol{n}(\%)$ & $\mathbf{n}$ & Serous $\boldsymbol{n}(\%)$ \\
\hline Russo et al, $2009^{8}$ & 543 & $296(55 \%)$ & 81 & $43(53 \%)$ \\
Bolton et al, $2012^{24}$ & 909 & $617(68 \%)$ & 304 & $213(70 \%)$ \\
Mavaddat et al, $2012^{25}$ & 813 & $534(66 \%)$ & 272 & $191(70 \%)$ \\
\hline Total & 2265 & $1447(64 \%)$ & 657 & $447(68 \%)$ \\
\hline
\end{tabular}

\section{Model design and analyses}

Chen et al.' presented ovarian cancer risk estimates per 10-year intervals (e.g., cumulative risk for ovarian cancer at age 50 assuming no cancer at age 20). To transform these 10-year risks into yearly cumulative risk estimates, we used nonparametric interpolating splines. The resulting risks were used to calculate cumulative hazards for each age $(t)$ as $H(t)=-\log (S(t))$, where log is the natural logarithm and $S(t)$ is the probability that cancer has not occurred at age $t$. Hazards $h(t)$ for age $t$ were calculated as the increment between $\mathrm{H}(t-1)$ and $\mathrm{H}(t)$. The hazards were multiplied with the hypothesized HRs (Table 4): if risk-reducing salpingectomy took place at age $t_{\text {RRS }}$ and delayed riskreducing oophorectomy took place at age $t_{R R O^{\prime}}$ the hazards between ages $t_{R R S}$ and $t_{R R O}\left(>t_{R R S}\right.$ and $\leq t_{R R O}$ ) were multiplied with the assumed HR for risk-reducing salpingectomy (e.g., 0.35 in case of $65 \%$ risk reduction or 1.0 in case of no risk reduction), and the hazards corresponding to ages $>t_{\text {RRO }}$ were multiplied with the assumed HR for risk-reducing salpingo-oophorectomy (i.e., 0.04 in case of $96 \%$ risk reduction or 0.20 in case of $80 \%$ risk reduction). New cumulative risks were calculated as 1 minus the exponentiated negative sum of the appropriate hazards, that is, $1-\exp \left(-\Sigma h_{i}\right)$, where $i$ denotes the relevant ages. For example, if a 35-year-old woman presents without ovarian cancer, 
and risk-reducing salpingo-oophorectomy will take place at age 45 years with $80 \%$ risk reduction (HR 0.20), her estimated cumulative risk for ovarian cancer at age 70 is based on the hazards from age 36 years up to and including age 70 years, where the hazards from age 46 years onwards are multiplied with 0.20. The model design is illustrated in Figure 1.

Table 4. Overview of hazard ratios used in the Cox proportional hazard model

\begin{tabular}{lcc}
\hline & \multicolumn{2}{c}{ Model input } \\
\cline { 2 - 3 } Parameter & Best case & Worst case \\
\hline Risk reduction of ovarian cancer by RRSO or RRO & $H R 0.04^{23}$ & $H R 0.20^{2,3}$ \\
Assumed risk reduction of ovarian cancer by RRS & $H R 0.35^{8,24,25}$ & $H R 1.0$ \\
\hline
\end{tabular}

Abbreviations: RRSO, risk-reducing salpingo-oophorectomy; RRO, risk-reducing oophorectomy; RRS, riskreducing salpingectomy;

Twenty scenarios were run for BRCA1 with five explicitly chosen combinations of risk-reducing salpingectomy (risk reduction $65 \%$ or $0 \%$ ) between age 33 and 40 years and second-stage riskreducing oophorectomy (risk reduction $96 \%$ or $80 \%$ ) between age 40 and 45 years. For BRCA2, 28 scenarios were run with seven different combinations of risk-reducing salpingectomy (risk reduction $65 \%$ or $0 \%$ ) between age 33 and 43 years and second-stage risk-reducing oophorectomy (risk reduction $96 \%$ or $80 \%$ ) between age 45 and 50 years. More scenarios for BRCA2 were run because the interval between completion of childbearing and recommended age for risk-reducing salpingo-oophorectomy is longer in BRCA2 mutation carriers.

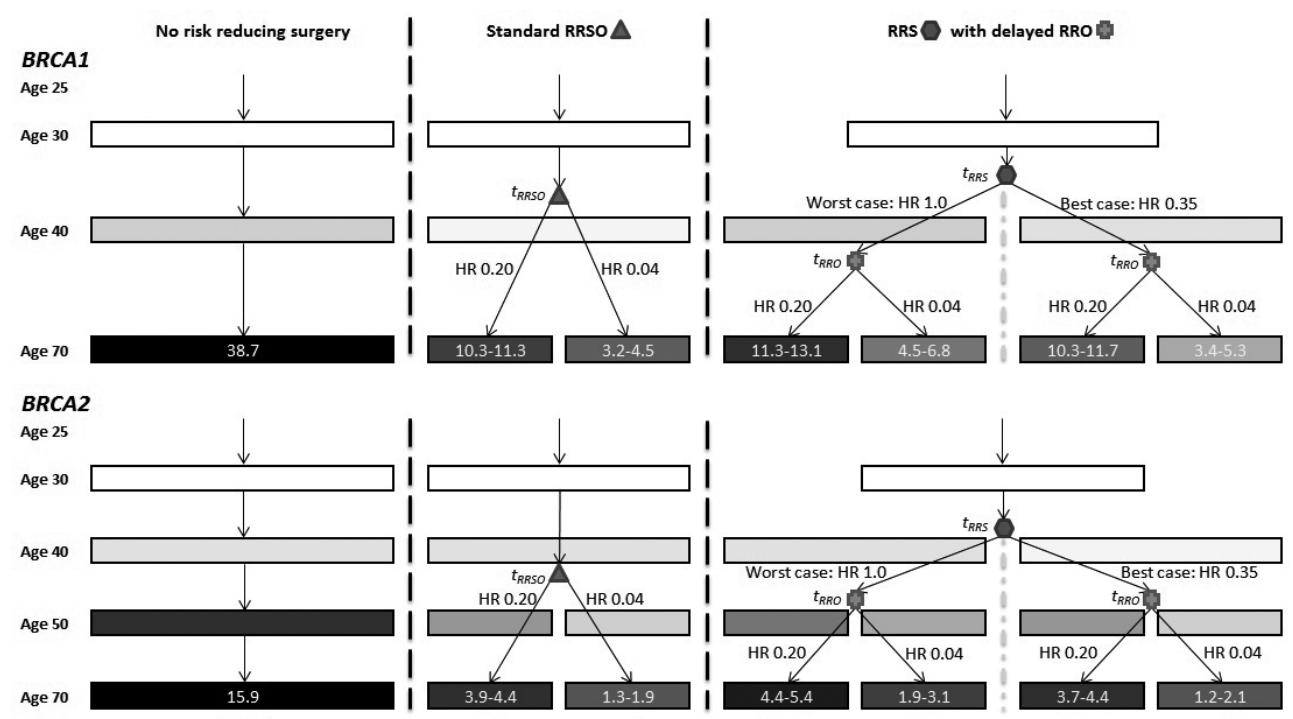

Figure 1. Overview of model for each risk-reducing strategy with assumed hazard ratios per branch. The darker the field, the higher the mean cumulative ovarian cancer risk. Similar colors represent comparable risks at each age. The range of point estimates of the mean cumulative risk at age 70 is given for a mutation carrier who is currently 25 years old, and includes point estimates for different ages at risk-reducing surgeries and various intervals between them (if applicable). 
Because no human subjects were involved in this study, no informed consent was needed and the study was exempt from application to and approval of the institutional review board according to Dutch law.

\section{RESULTS}

\section{BRCA1}

BRCA1 mutation carriers' cumulative risks of ovarian cancer at age 70 for several scenarios are provided in Table 5, conditional on the current age. Estimated cumulative risks assuming both 96\% and $80 \%$ risk reduction by either risk-reducing salpingo-oophorectomy or delayed risk-reducing oophorectomy are displayed with corresponding 95\% Cls.

Postponing oophorectomy from age 38 to 43, for example, will increase the point estimate of a 30-year-old BRCA 1 mutation carrier's cumulative risk from 3.4\% to 5.2\% (assuming 96\% risk reduction of risk-reducing salpingo-oophorectomy) or from 10.4\% to $11.8 \%$ (assuming $80 \%$ risk reduction of salpingo-oophorectomy) in case there is no effect of previous salpingectomy. In case risk-reducing salpingectomy at age 33 years already reduces her risk by 65\%, point estimates will decrease from $3.4 \%$ to $3.3 \%$ (96\% risk reduction of salpingo-oophorectomy) or from $10.4 \%$ to $10.0 \%$ (80\% risk reduction of salpingo-oophorectomy). Differences in risk estimates for this example assuming 96\% risk reduction are displayed in Figure $2 \mathrm{~A}$.

In a second example, a 35-year-old BRCA1 mutation carrier considers standard risk-reducing salpingo-oophorectomy at short term or risk-reducing salpingectomy at short term with delayed oophorectomy at age 40 years. Risk estimates for this example are illustrated in Figure 2B for an assumed $96 \%$ risk reduction of salpingo-oophorectomy.

The maximum increase in point estimates (from 1.8\% to 4.1\%) occurs in a 40-year-old BRCA1 mutation carrier undergoing oophorectomy at age 45 years after nonprotective salpingectomy instead of salpingo-oophorectomy (96\% risk reduction) at age 40 years. Assuming 65\% risk reduction by salpingectomy and $96 \%$ of salpingo-oophorectomy, point estimates either increase (maximum increase from $1.8 \%$ to $2.6 \%$ ) or decrease (maximum decrease from $3.4 \%$ to $3.3 \%$ ) depending on current age and intervals between surgeries. 


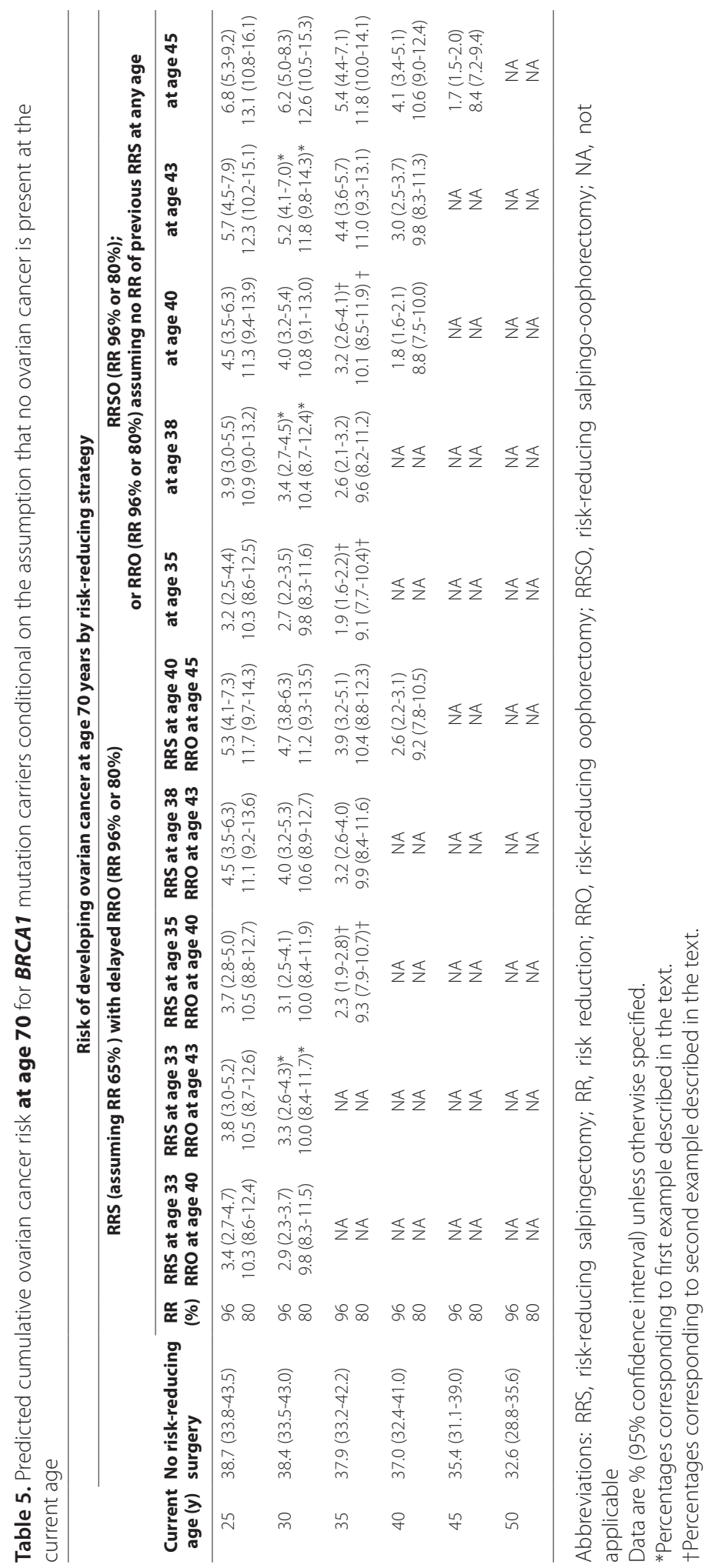



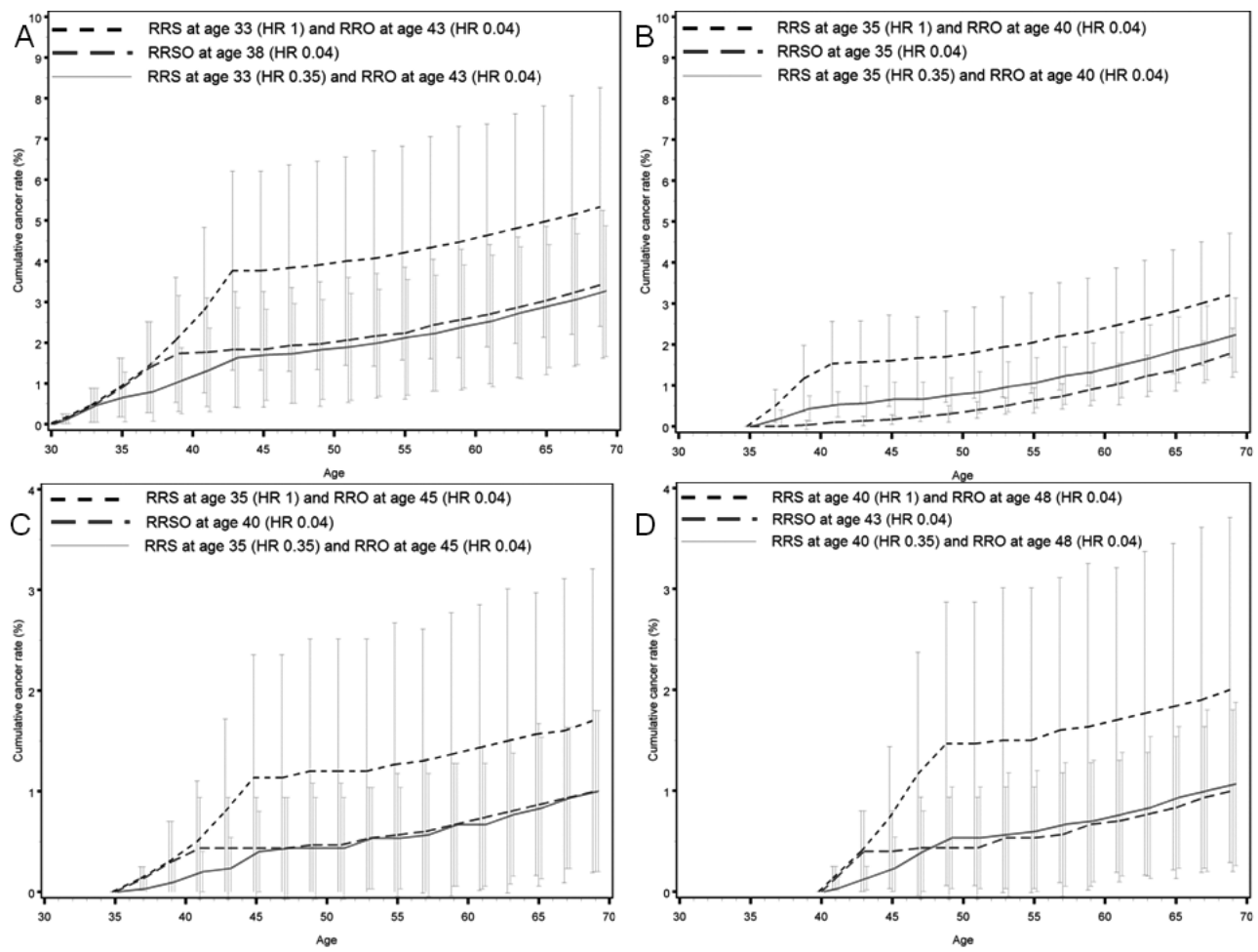

Figure 2. Estimated cumulative ovarian cancer risk (point estimates and $95 \%$ confidence intervals) for currently unaffected BRCA1 ( $A$ en $B$ ) and BRCA2 (C and D) mutation carriers. Abbreviations: RRS, risk-reducing salpingectomy; RRSO, risk-reducing salpingo-oophorectomy; RRO, risk-reducing oophorectomy.

\section{BRCA2}

Table 6 provides cumulative risks of ovarian cancer at age 70 years for several scenarios for BRCA2 mutation carriers conditional on the current age. Estimated cumulative risks assuming both 96\% and $80 \%$ risk reduction by either risk-reducing salpingo-oophorectomy or delayed risk-reducing oophorectomy are displayed with 95\% Cls.

For example, postponing oophorectomy from age 40 to 45 years will increase the point estimate of a 35-year-old BRCA2 mutation carrier's cumulative risk from 1.0\% to 1.7\% (assuming 96\% risk reduction of salpingo-oophorectomy) or from 3.6\% to 4.2\% (assuming $80 \%$ risk reduction of salpingo-oophorectomy) in case there is no effect of previous salpingectomy. In case risk-reducing salpingectomy at age 35 years already reduces her risk by 65\%, point estimates will be equal assuming 96\% risk reduction of salpingo-oophorectomy and increase from 3.5\% to 3.6\% assuming $80 \%$ risk reduction of salpingo-oophorectomy. Differences in risk estimates for this example assuming 96\% risk reduction of salpingo-oophorectomy are displayed in Figure 2C. 


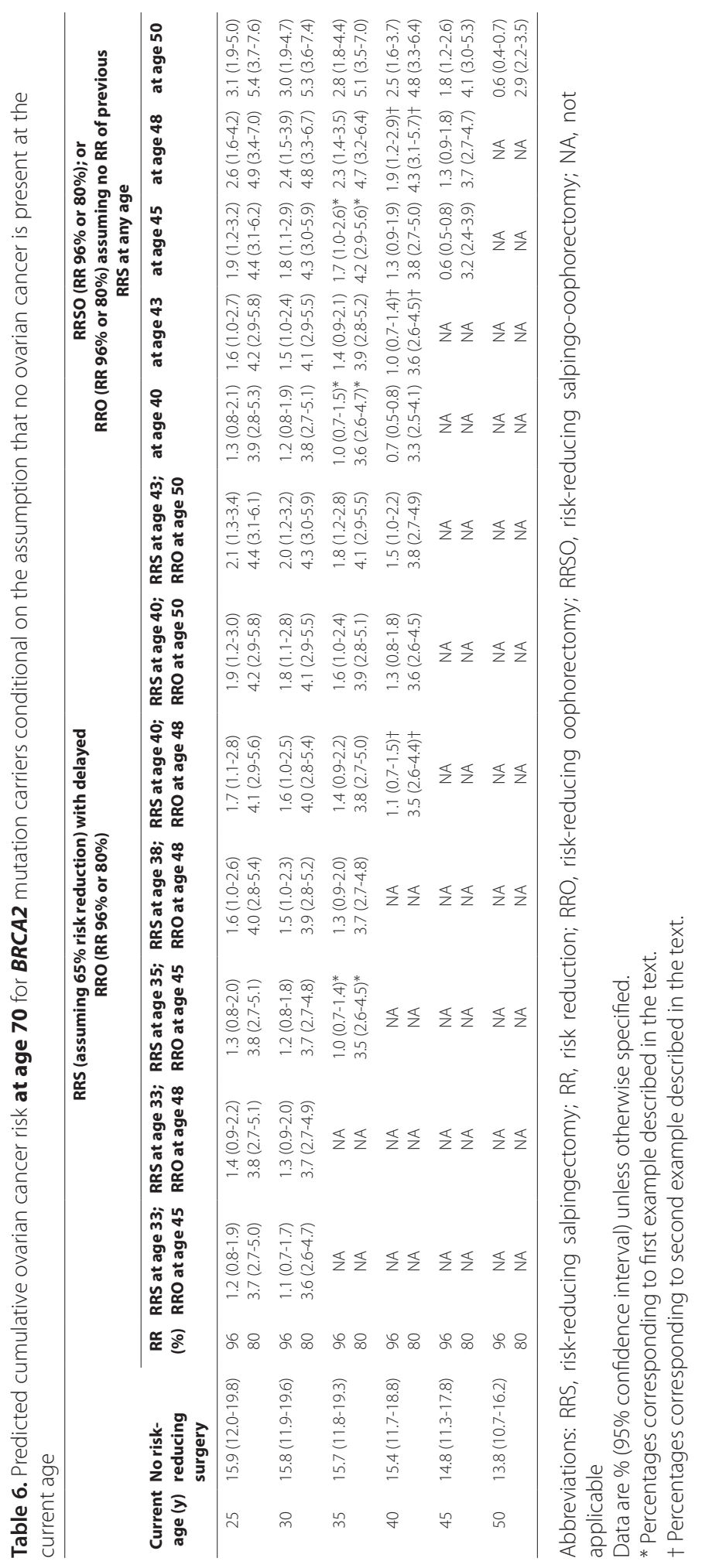


In a second example, a 40-year-old BRCA2 mutation carrier considers standard risk-reducing salpingo-oophorectomy at age 43 years or risk-reducing salpingectomy at short term with delayed risk-reducing oophorectomy at age 48 years. Risk estimates for this example are illustrated in Figure 2D for an assumed 96\% risk reduction of salpingo-oophorectomy.

In the worst-case scenario for BRCA2, point estimates maximally increase from $0.6 \%$ to $1.8 \%$ in 45-year-old carriers when oophorectomy is performed at age 50 years instead of risk-reducing salpingo-oophorectomy at age 45 years. Assuming 65\% risk reduction of salpingectomy, and 96\% of salpingo-oophorectomy, point estimates either increase (maximum increase from 1.3\% to 1.5\%) or decrease (maximum decrease from 1.5\% to 1.3\%) depending on current age and age at surgery.

\section{DIScussion}

In this study, cumulative risks of ovarian cancer for several risk-reducing strategies in BRCA1/2 mutation carriers are estimated by modeling data from the literature. Because risk-reducing salpingectomy with delayed oophorectomy has not been clinically studied yet, risk reduction rates for salpingectomy were estimated by combining existing data: 65\% (best-case) or 0\% (worst-case). In the best-case scenario, risk estimates were very similar for risk-reducing salpingooophorectomy at the currently recommended age and for risk-reducing salpingectomy with delayed oophorectomy 5 years beyond the currently recommended age. In the worst-case scenario (assuming no risk-reducing effect of salpingectomy), delaying oophorectomy by 5 years is estimated to maximally increase cumulative risk point estimates by 2.3 (BRCA1) and 1.2 (BRCA2) percentage points compared with risk-reducing salpingo-oophorectomy. Oophorectomy remains part of every scenario and none of the presented risk estimates are for salpingectomy alone.

Two research groups have published simulation models for risk-reducing surgery in BRCA1/2 mutation carriers since 2010. ${ }^{16,27}$ Kurian et al. ${ }^{27}$ published a Monte Carlo simulation model as an online tool for cancer incidences and survival under risk-reducing mastectomy and risk-reducing salpingo-oophorectomy at different ages. For scenarios matching ours, results lie within the same range. They conclude that early mastectomy and salpingo-oophorectomy most effectively prevent cancer. However, alternative strategies resulted in similar survival rates, albeit reducing cancer incidence less substantially.

Kwon et al. ${ }^{16}$ published the only model that included risk-reducing salpingectomy (at age 40 years, HR 0.40) with delayed oophorectomy (at age 50 years) besides risk-reducing salpingo-oophorectomy (at age 40 years, HR 0.20 for ovarian and HR 0.30-0.60 for breast cancer). They calculated gain in life expectancy and quality-adjusted life-years using a Markov model with Monte Carlo simulations. Risk-reducing salpingo-oophorectomy at age 40 years offered the greatest risk reduction for ovarian and breast cancer, but risk-reducing salpingectomy with delayed oophorectomy was costeffective considering quality-adjusted life expectancy and therefore a reasonable alternative for 
$B R C A 1 / 2$ mutation carriers who are reluctant to undergo risk-reducing salpingo-oophorectomy. $B R C A 1 / 2$ mutation carriers' choice for risk-reducing strategies is highly personal. To make an informed choice between risk-reducing salpingo-oophorectomy and risk-reducing salpingectomy with delayed oophorectomy, knowledge of estimated ovarian cancer risks under several scenarios is essential. BRCA mutation carriers should decide themselves which consequence outweighs the other: the possibility of slightly higher estimated ovarian cancer risks or the acute onset of surgical menopause and its consequences. The main strength of our model is that we provide such risk estimates for a number of potential scenarios: surgery at several ages and with various time intervals between risk-reducing salpingectomy and oophorectomy. Consequently, the risk estimates generated by our model can contribute to the decision for or against the alternative strategy. Another strength is that we have taken into account two different risk reduction rates for risk-reducing salpingo-oophorectomy: 80\% (two meta-analyses) and 96\% (best ever reported). 2,3,23 Although halving of breast cancer risk by premenopausal risk-reducing salpingo-oophorectomy was widely communicated until recently, ${ }^{2}$ we did not include the effect of salpingo-oophorectomy on breast cancer in our model. Heemskerk-Gerritsen et al. ${ }^{6}$ found no beneficial effect of riskreducing salpingo-oophorectomy on breast cancer after applying alternative analyses to earlier large cohort studies, thereby minimizing amounts of bias. Furthermore, the effect of salpingooophorectomy is irrelevant for the substantial part (up to 32\%) of BRCA1/2 mutation carriers having already undergone mastectomy by age $40-45$ years. ${ }^{28}$

The most important limitation of our study is the uncertainty about assumptions the model was built on. First, reported penetrance of ovarian cancer at different ages in BRCA1/2 mutation carriers widely differs. ${ }^{15,29}$ This might be the result of methodologic differences and differences in patient populations. If penetrance appears to be higher in reality than reported by Chen et al., ${ }^{1}$ our model might underestimate absolute differences in cumulative risks. Second, given the lack of previous studies on its efficacy, risk reduction estimates for salpingectomy were totally based on the hypothesis that all serous ovarian carcinomas in BRCA1/2 mutation carriers could be prevented by salpingectomy. The $58 \%$ risk reduction of ovarian cancer after tubal ligation in BRCA1 mutation carriers could be considered indirect evidence. ${ }^{30}$

Based on the presented risk estimates, we conclude that risk-reducing salpingectomy necessarily followed by delayed oophorectomy may be offered to BRCA1/2 mutation carriers as an alternative strategy to risk-reducing salpingo-oophorectomy, preferably within a clinical trial. With current age as well as timing and type of surgeries taken into account, these ovarian cancer risk estimates are highly personalized. Providing these personal risk estimates, our model may support both patients and physicians in shared decision-making, and this may contribute to individualized health care. A decision aid might be helpful to comprehensibly present these risk estimates to patients. 


\section{REFEREMCES}

1. Chen S, Parmigiani G. Meta-analysis of BRCA1 and BRCA2 penetrance. J Clin Oncol 2007; 25(11): 1329-33.

2. Rebbeck TR, Kauff ND, Domchek SM. Meta-analysis of risk reduction estimates associated with riskreducing salpingo-oophorectomy in BRCA1 or BRCA2 mutation carriers. J Nat/ Cancer Inst 2009; 101(2): 80-7.

3. Marchetti C, De Felice F, Palaia I, et al. Risk-reducing salpingo-oophorectomy: a meta-analysis on impact on ovarian cancer risk and all cause mortality in BRCA 1 and BRCA 2 mutation carriers. BMC Womens Health 2014; 14(1): 150.

4. Fakkert IE, Mourits MJ, Jansen L, et al. Breast Cancer Incidence After Risk-Reducing SalpingoOophorectomy in BRCA1 and BRCA2 Mutation Carriers. Cancer Prev Res (Phila) 2012; 5(11): 1291-7.

5. Mavaddat N, Peock S, Frost D, et al. Cancer risks for BRCA1 and BRCA2 mutation carriers: results from prospective analysis of EMBRACE. J Natl Cancer Inst 2013; 105(11): 812-22.

6. Heemskerk-Gerritsen BA, Seynaeve C, van Asperen CJ, et al. Breast cancer risk after salpingooophorectomy in healthy BRCA1/2 mutation carriers: revisiting the evidence for risk reduction. J Nat/ Cancer Inst 2015; 107(5).

7. Shuster LT, Gostout BS, Grossardt BR, Rocca WA. Prophylactic oophorectomy in premenopausal women and long-term health. Menopause Int 2008; 14(3): 111-6.

8. Russo A, Calo V, Bruno L, Rizzo S, Bazan V, Di Fede G. Hereditary ovarian cancer. Crit Rev Oncol Hematol 2009; 69(1): 28-44.

9. Arts-de Jong M, Maas AH, Massuger LF, Hoogerbrugge N, de Hullu JA. BRCA1/2 mutation carriers are potentially at higher cardiovascular risk. Crit Rev Oncol Hematol 2014.

10. Parker WH, Feskanich D, Broder MS, et al. Long-term mortality associated with oophorectomy compared with ovarian conservation in the nurses' health study. Obstet Gynecol 2013; 121(4): 709-16.

11. Piek JM, van Diest PJ, Zweemer RP, et al. Dysplastic changes in prophylactically removed Fallopian tubes of women predisposed to developing ovarian cancer. J Pathol 2001; 195(4): 451-6.

12. Mingels MJ, Roelofsen T, van der Laak JA, et al. Tubal epithelial lesions in salpingo-oophorectomy specimens of BRCA-mutation carriers and controls. Gynecol Oncol 2012; 127(1): 88-93.

13. Kurman RJ. Origin and molecular pathogenesis of ovarian high-grade serous carcinoma. Ann Oncol 2013; 24 Suppl 10: x16-21.

14. Walker JL, Powell CB, Chen LM, et al. Society of Gynecologic Oncology recommendations for the prevention of ovarian cancer. Cancer 2015.

15. Greene MH, Mai PL, Schwartz PE. Does bilateral salpingectomy with ovarian retention warrant consideration as a temporary bridge to risk-reducing bilateral oophorectomy in BRCA1/2 mutation carriers? Am J Obstet Gynecol 2011; 204(1): 19 e1-6.

16. Kwon JS, Tinker A, Pansegrau G, et al. Prophylactic salpingectomy and delayed oophorectomy as an alternative for BRCA mutation carriers. Obstet Gynecol 2013; 121(1): 14-24.

17. Anderson CK, Wallace S, Guiahi M, Sheeder J, Behbakht K, Spillman MA. Risk-reducing salpingectomy as preventative strategy for pelvic serous cancer. Int J Gynecol Cancer 2013; 23(3): 417-21.

18. Holman LL, Friedman S, Daniels MS, Sun CC, Lu KH. Acceptability of prophylactic salpingectomy with delayed oophorectomy as risk-reducing surgery among BRCA mutation carriers. Gynecol Oncol 2014; 133(2): 283-6.

19. Arts-de Jong M, Harmsen MG, Hoogerbrugge N, Massuger LF, Hermens RP, de Hullu JA. Risk-reducing salpingectomy with delayed oophorectomy in BRCA1/2 mutation carriers: patients' and professionals' perspectives. Gynecol Oncol 2015; 136(2): 305-10.

20. Chandrasekaran D, Menon U, Evans G, et al. Risk reducing salpingectomy and delayed oophorectomy in high risk women: views of cancer geneticists, genetic counsellors and gynaecological oncologists in the UK. Fam Cancer 2015. 
21. Powell CB, Kenley E, Chen LM, et al. Risk-reducing salpingo-oophorectomy in BRCA mutation carriers: role of serial sectioning in the detection of occult malignancy. J Clin Oncol 2005; 23(1): 127-32.

22. Medeiros $F$, Muto MG, Lee $Y$, et al. The tubal fimbria is a preferred site for early adenocarcinoma in women with familial ovarian cancer syndrome. Am J Surg Pathol 2006; 30(2): 230-6.

23. Rebbeck TR, Lynch HT, Neuhausen SL, et al. Prophylactic oophorectomy in carriers of BRCA1 or BRCA2 mutations. N Engl J Med 2002; 346(21): 1616-22.

24. Bolton KL, Chenevix-Trench G, Goh C, et al. Association between BRCA1 and BRCA2 mutations and survival in women with invasive epithelial ovarian cancer. JAMA : the journal of the American Medical Association 2012; 307(4): 382-90.

25. Mavaddat N, Barrowdale D, Andrulis IL, et al. Pathology of breast and ovarian cancers among BRCA1 and BRCA2 mutation carriers: results from the Consortium of Investigators of Modifiers of BRCA1/2 (CIMBA). Cancer Epidemiol Biomarkers Prev 2012; 21 (1): 134-47.

26. Alsop K, Fereday S, Meldrum C, et al. BRCA mutation frequency and patterns of treatment response in BRCA mutation-positive women with ovarian cancer: a report from the Australian Ovarian Cancer Study Group. J Clin Oncol 2012; 30(21): 2654-63.

27. Kurian AW, Munoz DF, Rust P, et al. Online tool to guide decisions for BRCA1/2 mutation carriers. J Clin Oncol 2012; 30(5): 497-506.

28. Evans DG, Lalloo F, Ashcroft L, et al. Uptake of risk-reducing surgery in unaffected women at high risk of breast and ovarian cancer is risk, age, and time dependent. Cancer Epidemiol Biomarkers Prev 2009; 18(8): 2318-24.

29. Evans DG, Shenton A, Woodward E, Lalloo F, Howell A, Maher ER. Penetrance estimates for BRCA1 and BRCA2 based on genetic testing in a Clinical Cancer Genetics service setting: risks of breast/ovarian cancer quoted should reflect the cancer burden in the family. BMC Cancer 2008; 8: 155.

30. Antoniou AC, Rookus M, Andrieu N, et al. Reproductive and hormonal factors, and ovarian cancer risk for BRCA1 and BRCA2 mutation carriers: results from the International BRCA1/2 Carrier Cohort Study. Cancer Epidemiol Biomarkers Prev 2009; 18(2): 601-10. 

$\therefore$

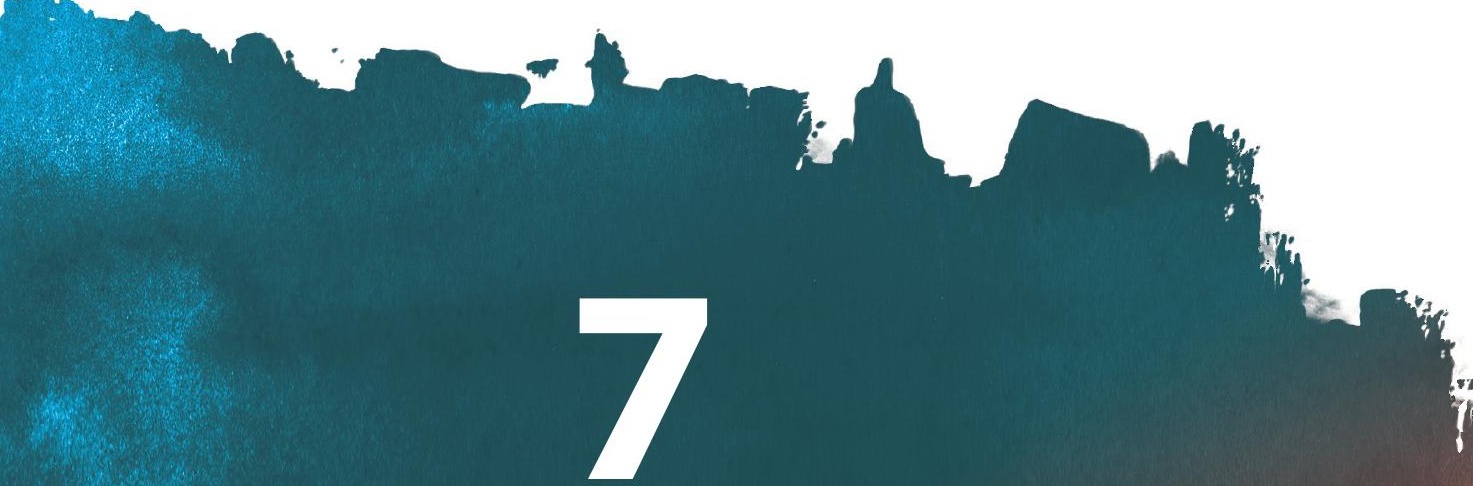

Early salpingectomy (Tllbectomy) with delayed oophorectomy to improve quality of life as alternative for niskreducing salpingo-oophorectomy in BRCA1/2 mutation carriens (TUBA study): a prospective now-randomized multicenter study

Marline G. Harmsen, Marieke Arts-de Jong, N. Hoogerbrugge, Angela H.E.M. Maas, Judith B. Prins, Johan Bulten, Steven Teerenstra, Eddy M. Adang, Jurgen M.J. Piek, Helena C. van Doorn, Marc van Beurden, Marian J.E. Mourits, Ronald P. Zweemer, Katja N. Gaarenstroom, Brigitte F.M. Slangen, M. Caroline Vos, Luc R.C.W. van Lonkhuijzen, Leon F.A.G. Massuger, Rosella P.M.G. Hermens, Joanne A. de Hullu 


\section{ABSTRACT}

\section{Background}

Risk-reducing salpingo-oophorectomy (RRSO) around the age of 40 is currently recommended to BRCA1/2 mutation carriers. This procedure decreases the elevated ovarian cancer risk by 80-96\% but it initiates premature menopause as well. The latter is associated with short-term and longterm morbidity, potentially affecting quality of life (QoL). Based on recent insights into the fallopian tube as possible site of origin of serous ovarian carcinomas, an alternative preventive strategy has been put forward: early risk-reducing salpingectomy (RRS) and delayed oophorectomy (RRO). However, efficacy and safety of this alternative strategy have to be investigated.

\section{Methods}

A multicenter non-randomized trial in 11 Dutch centers for hereditary cancer will be conducted. Eligible patients are premenopausal BRCA1/2 mutation carriers after completing childbearing without (a history of) ovarian carcinoma. Participants choose between standard RRSO at age 3540 (BRCA1) or 40-45 (BRCA2) and the alternative strategy (RRS upon completion of childbearing and RRO at age 40-45 (BRCA1) or 45-50 (BRCA2)). Women who opt for RRS but do not want to postpone RRO beyond the currently recommended age are included as well. Primary outcome measure is menopause-related QoL. Secondary outcome measures are ovarian/breast cancer incidence, surgery-related morbidity, histopathology, cardiovascular risk factors and diseases, and cost-effectiveness. Mixed model data analysis will be performed.

\section{Discussion}

The exact role of the fallopian tube in ovarian carcinogenesis is still unclear. It is not expected that further fundamental research will elucidate this role in the near future. Therefore, this clinical trial is essential to investigate RRS with delayed RRO as alternative risk-reducing strategy in order to improve QoL.

\section{Trial registration}

ClinicalTrials.gov (NCT02321228) 


\section{BACKGROUHD}

\section{BRCA germline mutations and ovarian cancer}

Epithelial ovarian cancer is the most lethal malignancy of the female genital tract. With respect to treatment and prognosis, primary carcinomas of the ovaries, fallopian tubes and peritoneum are considered one disease entity often referred to as 'ovarian carcinoma'. Women with germline mutations in one of the two BRCA genes are at increased risk of developing breast and ovarian cancer. Cumulative breast cancer risks are estimated 57-65\% (95\% Cl: 44-78\%) for BRCA1 and 4549\% (95\% Cl: 31-57\%) for BRCA2 mutation carriers by age 70, whereas cumulative ovarian cancer risks lie around 39-40\% (95\% Cl: 18-54\%) and 11-18\% (95\% Cl: 2.4-23\%) by the age of 70 for $B R C A 1$ and BRCA2, respectively. ${ }^{1,2}$ Ovarian carcinoma occurs at younger age in BRCA1 mutation carriers than in BRCA2 mutation carriers or the general population (both mean and median 51 versus 56 versus 60 years respectively). ${ }^{3}$ In BRCA1/2 germline mutation carriers, approximately $65 \%$ of all ovarian carcinomas are of the serous subtype..$^{4-6}$

\section{Risk-reducing salpingo-oophorectomy (RRSO)}

In contrast to breast cancer surveillance, screening for ovarian cancer has been highly ineffective. ${ }^{7-9}$ Therefore, the only intervention to reduce ovarian cancer risk is risk-reducing salpingooophorectomy (RRSO), which decreases ovarian cancer incidence by about 80-96\%.,10-12 However, this effect might be underestimated due to studies that included women who underwent oophorectomy alone and/or underwent surgery above the currently recommended age: 35-40 for BRCA1 and 40-45 for BRCA2 mutation carriers.,11,13 The residual risk of primary peritoneal cancer after RRSO is approximately 1\%; however, it was also reported to be more than $4 \%{ }^{10,14-16}$ RRSO is often laparoscopically performed at an outpatients' department. Serious surgical complications rates are low. 4,17,18 Main adverse effects of RRSO are related to premature surgical menopause, including short-term effects like vasomotor symptoms (i.e. hot flushes), sleep disturbances, vaginal dryness and sexual symptoms. ${ }^{4}$ Long-term effects include osteoporosis, increased risk of cardiovascular disease, cognitive impairment and increased depressive and anxiety symptoms, although prospective studies on these long-term effects in BRCA mutation carriers in particular are not available., ${ }^{419-22}$ Postsurgical hormone replacement therapy (HRT) does not fully alleviate climacteric and sexual symptoms. ${ }^{20,23}$ The reduction of breast cancer incidence by half achieved by performing RRSO at premenopausal age ${ }^{11,12}$ has recently become arguable ${ }^{24-26}$ and therefore questionable as motivation to undergo RRSO.

\section{Role of fallopian tube in "ovarian" carcinogenesis}

Based on recent scientific insights, the fallopian tube is considered the most important site of origin of pelvic high grade serous carcinoma nowadays..$^{27-31}$ It is suggested that benign tubal epithelium can transform into serous tubal intraepithelial carcinoma (STIC) or invasive tubal carcinoma. ${ }^{32}$ The 
(pre)malignant cells can exfoliate from the tubal epithelial lining and migrate to the ovary and abdominal cavity. This theory is based on several findings. First, no clear precursor of ovarian cancer has been found in the ovary itself. Second, earlier studies showed the presence of STIC in 36-60\% of sporadic pelvic serous carcinomas ${ }^{33-35}$ which harbored identical mutation in the TP53 gene to the cells of concurrent pelvic serous carcinomas in 92\%. ${ }^{36}$ Third, pelvic serous carcinoma cells resemble tubal lining epithelium more than ovarian surface epithelium. ${ }^{37}$ Several investigators focused on prophylactically removed fallopian tubes of germline BRCA1/2 mutation carriers, showing the presence of STIC in about 4\% (range 0-12\%); ;4,38-47 nearly all STICS were localised in the tubal distal fimbrial ends. ${ }^{34}$

\section{Innovative preventive strategy: risk-reducing salpingectomy (RRS) with delayed oophorectomy (RRO)}

The growing evidence of the role of the fallopian tube in the origin of serous ovarian carcinoma together with the disadvantages of premature surgical menopause caused by RRSO underlie the need for an alternative risk-reducing strategy. RRS upon completion of childbearing offers an early, potentially risk-reducing intervention; however, it is still uncertain whether and to what extent the risk of ovarian cancer will be reduced. Furthermore, around $68 \%$ of occult carcinomas are found in tubes ${ }^{48}$ and could now be detected at an early stage. The main advantage of delaying subsequent RRO beyond the currently recommended age will be postponement of premature menopause and its effect on noncancer-related morbidity and (menopause-related) quality of life (QoL). Several authors previously suggested this innovative strategy ${ }^{48-50}$ and a feasibility study among both professionals and germline BRCA1/2 mutation carriers from our group showed a broad national support to evaluate this new strategy in a prospective study. ${ }^{51}$

\section{Objective}

The aim of this study is to determine whether an innovative risk-reducing strategy, consisting of RRS upon completion of childbearing with delayed RRO, results in better menopause-related QoL without increase of ovarian and breast cancer risk in germline BRCA1/2 mutation carriers compared to standard treatment, consisting of RRSO at currently recommended age.

\section{METHODS/DESIGH}

\section{Study design}

We will perform a nationwide prospective non-randomized multicenter trial in 11 hospitals with a department for hereditary cancer. Eligible patients will have the opportunity to choose for standard or innovative strategy. Women who opt for RRS but do not want to postpone RRO beyond the currently recommended age or are unsure about this at enrollment are included 
as well; however, they will not contribute to the number of inclusions needed according to the sample size calculation. See Figure 1 for an overview of the study design.

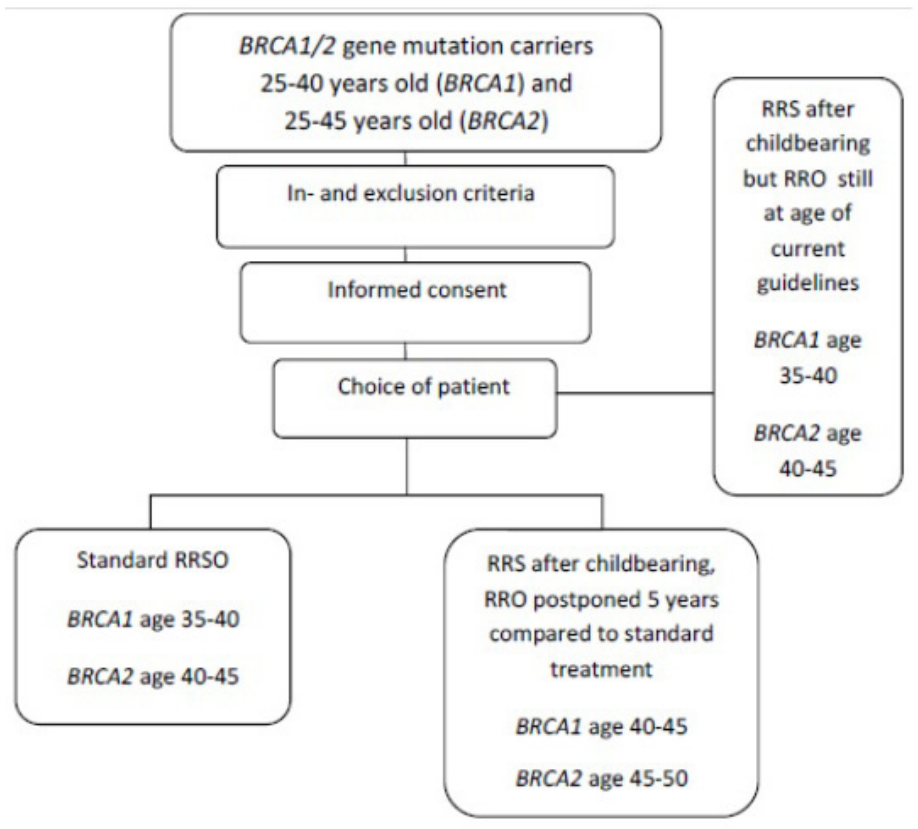

Figure 1. TUBA study design

Although a randomized controlled trial would be the preferred study design, an earlier published feasibility study among healthcare professionals and germline BRCA1/2 mutation carriers showed that randomization would be an insurmountable barrier for participation in a clinical study. ${ }^{11}$ These women want to decide themselves on their risk-reducing strategy and it is therefore unlikely that they will participate in a randomized controlled trial. Taken this into account, a prospective nonrandomized design seems the most appropriate, letting women the opportunity to decide for themselves.

In addition, a control group will be formed by women who underwent RRSO between 1 and 5 years ago, in order to compare QoL between study participants and women who did not have the opportunity to choose for an alternative treatment. 


\section{Study population}

a. Hospitals

Eleven hospitals with a hereditary cancer department will participate in this study, of which seven are university tertiary hospitals.

b. Patients

Women carrying a documented germline BRCA1/2 mutation from the department of Clinical Genetics or Hereditary Cancer of each hospital, who are between 25 and 40 (BRCA1) or 45 (BRCA2) years old without previous RRSO.

Inclusion criteria

- Premenopausal women with a documented BRCA1 and/or BRCA2 gene germline mutation

- Age 25-40 years for BRCA1 mutation carriers and 25-45 years for BRCA2

- Childbearing completed

- Presence of at least one fallopian tube

- Participants may have a personal history of non-ovarian malignancy

- Informed consent

Exclusion criteria

- Postmenopausal status (natural menopause or due to (cancer) treatment)

- Wish for second stage RRO within two years after RRS (if clear at enrollment)

- Legally incapable

- Prior bilateral salpingectomy

- A personal history of ovarian, fallopian tube or peritoneal cancer

- Evidence of malignant disease at enrollment

- Current treatment for malignant disease

- Inability to read or speak Dutch

c. Patient recruitment

Eligible women will be sent a letter to inform them on this study. BRCA1/2 mutation carriers will be asked to respond whether or not they would be interested to participate. If they are interested, the patient information form will be sent and an appointment will be made to explain the rationale, design and aims of the study in person. The patient will have sufficient time (at least one week) to consider the study before deciding to participate. Written informed consent from the patient is required before participation. Furthermore, every newly diagnosed germline BRCA1/2 mutation carrier at the department of Clinical Genetics that fulfills the inclusion criteria will be informed on the study.

\section{Outcome measures}

a. Primary outcome measure

Menopause-specific QoL, measured by the Greene Climacteric Scale (GCS) questionnaire 
in Dutch. ${ }^{52}$ This questionnaire consists of 21 items divided into various domains: psychological (11 items, divided into anxiety and depression subscales), somatic (7 items), vasomotor symptoms ( 2 items) and sexual (1 item). Each symptom is rated according to its severity using a four-point Likert scale. The Greene Climacteric score is the sum of all 21 items ranging from 0 to 63. A higher total score corresponds with more menopausal symptoms.

b. Secondary outcome measures

- General QoL and QoL-related items, measured by several questionnaires (see section Pre-treatment evaluation)

- Incidence of ovarian and breast cancer

- Surgical complications, e.g. infection, conversion, hemorrhage and complications at the second laparoscopic procedure (RRO) due to previous RRS.

- Histopathological findings of removed fallopian tubes and ovaries, i.e. (pre)malignancies

- Cardiovascular risk factors and incidence of cardiovascular disease

- Cost-effectiveness of the innovative treatment

\section{Interventions}

a. Standard treatment (control arm):

RRSO between age 35-40 in BRCA1 mutation carriers and between 40-45 in BRCA2 mutation carriers (exact ages vary across different hospitals) and when childbearing is completed.

b. Innovative treatment (experimental arm):

RRS when childbearing is completed with second stage RRO delayed for five years compared to the currently recommended age for RRSO, i.e. at the age of 40-45 in BRCA1 and 45-50 in BRCA2 mutation carriers. Regarding the definitive contraception which is a result of RRS and the age at which RRS is performed, women will be counseled in a similar manner as women consulting the gynecologist for sterilization. RRS will be performed according to Leblanc et al. ${ }^{53}$ Whenever a (pre)malignancy is found in the RRS specimen, RRO will be performed as soon as possible, as well as additional surgery or treatment if necessary, e.g. staging procedure.

\section{Data collection}

a. Pre-treatment evaluation

All patients will be asked to fill out web-based baseline questionnaires. Questionnaires on demographic data and medical history with a special focus on cancer and cardiovascular risk factors are included. Furthermore, QoL(-related) questionnaires include Dutch versions of the Greene Climacteric Scale (GCS), ${ }^{52}$ SF-36, ${ }^{54}$ EQ-5D-5 L, ${ }^{55}$ Cancer Worry Scale (CWS), ${ }^{56,57}$ Female Sexual Function Index (FSFI), ${ }^{58,59}$ Female Sexual Distress Scale (FSDS), ${ }^{59,60}$ 
and Decisional Conflict Scale (DCS). ${ }^{61}$ Questions based on the Institute of Medical Technology Assessment Productivity Cost Questionnaire (iPCQ) ${ }^{62}$ and the Medical Consumption Questionnaire (iMCQ) ${ }^{63}$ will be used to collect data on productivity loss and health consumption. Moreover, blood pressure, body mass index and waist-hip ratio will be documented. Fasting blood samples will be taken to measure cardiovascular risk factors.

b. Follow-up

Six weeks after surgery, data on surgical complications and histopathological findings are collected. The Sectioning and Extensively Examining the FIMbriated End (SEEFIM) of the fallopian tube (SEE-FIM) protocol will be used for the latter. ${ }^{64}$ Follow-up by web-based questionnaires as described at baseline except for the Decisional Conflict Scale is scheduled at 3 and 12 months after surgery. At 1, 5 and 15 years follow-up, the Decision Regret Scale (DRS) is added. ${ }^{65}$ From one year after surgery, questionnaires will be sent biennially until the end of follow-up (in case of only one surgery in the standard treatment arm) or until undergoing RRO (in the innovative arm). After RRO, data will be collected at six weeks and 3 and 12 months after surgery, comparable to follow-up after the first operation, and then biennial questionnaires will be sent until the end of followup, 15 years after the last (or only) surgery. Additionally, blood pressure, body mass index, waist-hip ratio and cardiovascular risk factors in fasting blood samples will be collected five years after each surgery. Follow-up by questionnaires will continue biennially until 15 years after the last surgery to detect occurrence of ovarian cancer. Since the wide possible range of age at inclusion, timing of surgeries and interval between surgeries, it is hard to specify and generalize the exact amount and timing of follow-up. A flowchart visualizing the follow-up schedule can be found in Figure 2.

c. Cost-effectiveness

This economic evaluation will compare costs and quality adjusted life years (QALY) of the innovative treatment with standard treatment. The perspective of this economic evaluation will be a societal perspective. Both healthcare and societal costs which can be related to this study will be assessed until 15 years after last surgery. Costs are collected on a per patient level. The incremental costs of innovative care compared to standard care will be based on the difference in costs between groups. The healthcare costs, measured by the Medical Consumption Questionnaire (iMCQ) tailored to this context, will be calculated. Societal costs will be calculated from a selection of the Productivity Cost Questionnaire (iPCQ). The output or consequences of both innovative and standard care will be determined by measuring QoL before and during the study. The SF-36 and EQ-5D-5 $L$ will be used for this analysis. The outcomes will be translated to a long-time difference in QALY. Key variables will be varied in a sensitivity analysis to evaluate their impact on the incremental costs per QALY gained ratio. Including the innovative strategy 
in future guideline recommendations depends on the incremental cost per QALY. As mentioned before, data will be collected until 15 years after last surgery. However, an interim cost-effectiveness analysis will be performed after eight years to provide information for proceeding implementation. Recommendation for implementation will be based on the empirical cost data completed with modeled costs over the remaining period. Accuracy of this model will be evaluated at the end of 15 years follow-up, when data collection on the actual costs during the remaining seven years will have been completed.

\section{d. Potential adverse events}

In the innovative treatment, BRCA1/2 mutation carriers will undergo an additional laparoscopy. Known complication rates for RRSO in a comparable population vary from 0.6-5\% for major complications (conversion, bladder or bowel injury, additional surgery required) and 3.7-10\% for minor complications (infection, bleeding, hematoma). 17,18,42,66 Risks might be lower for RRS alone. As mentioned before, data on surgery-related complications will be collected six weeks after each surgery. Furthermore, the worst-case scenario is that RRS does not reduce ovarian cancer risk at all. Then, the postponement of $\mathrm{RRO}$ for five years might result in a higher ovarian cancer incidence in the experimental arm. We used a model to calculate the risk for interval ovarian carcinoma when RRO is performed five years later than the current guideline age. The risk to develop ovarian carcinoma within these five years is estimated to be up to 1-2\% for BRCA1 mutation carriers and up to $0.5-1 \%$ for BRCA2 mutation carriers. Cancer incidence will be monitored by questionnaires. 


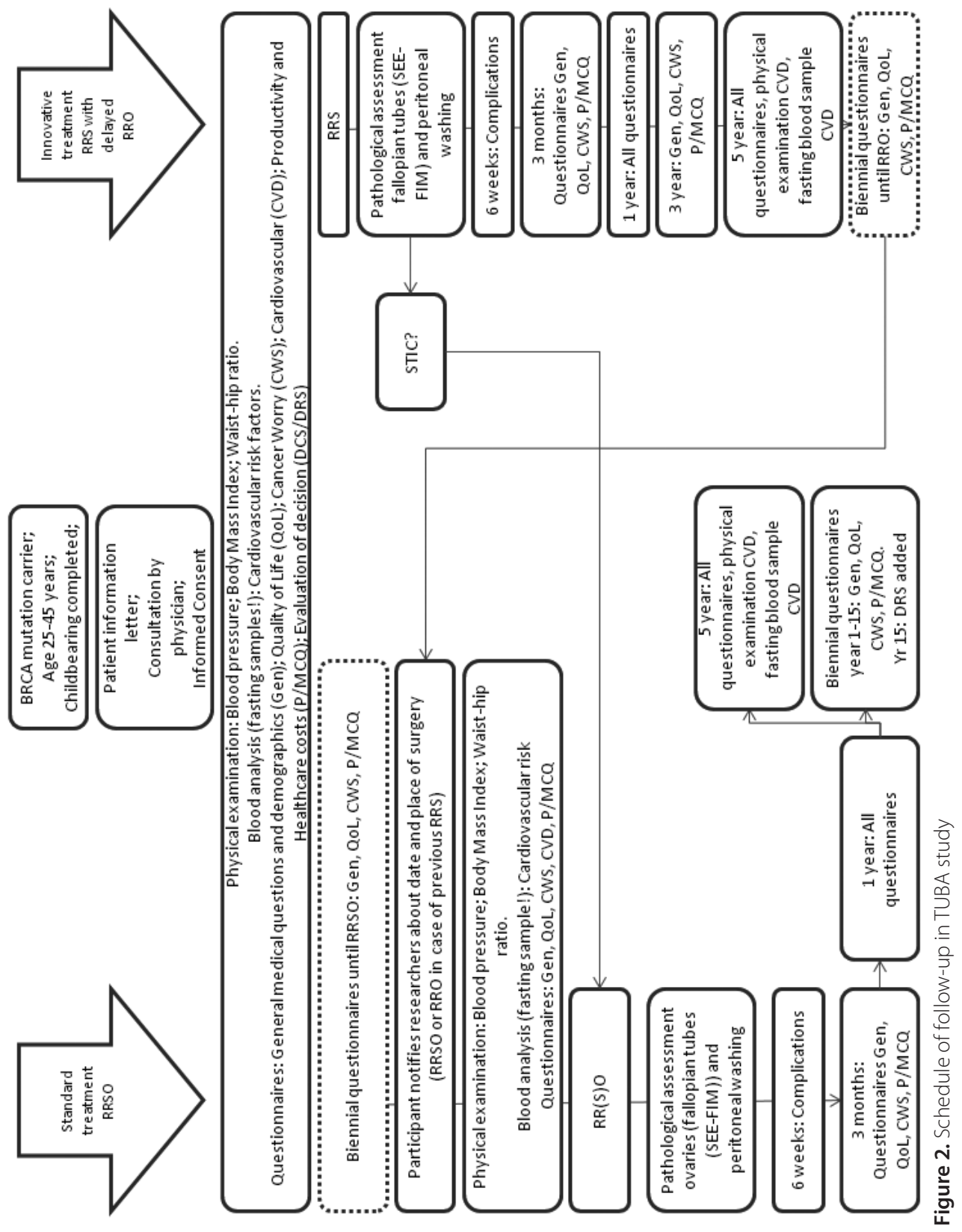




\section{Statistical analysis}

a. Sample size calculation

The primary outcome measure is menopause-specific QoL. Menopausal symptoms will be assessed by the Greene Climacteric Scale (GCS). The main comparison is the difference in GCS between women getting the innovative treatment and women getting standard RRSO without postsurgical hormone replacement therapy (HRT), which is about one third of women after RRSO. This difference is estimated at five points on the GCS, with standard deviation 7.36, based on figures of Barentsen et al. ${ }^{67}$ Each hospital will provide both innovative and standard treatment, based on patient choice (no randomization). We assume an intra-cluster correlation coefficient $\leq 0.10$. When we have about 10 hospitals, with 51 patients per hospital (total $n=510$ ), we expect that the majority of hospitals (7 hospitals or more) will provide at least 3 patients with the innovative treatment. The remaining hospitals ( 3 or less) provide 51 patients with standard treatment of whom 16 will be on RRSO without HRT. This scenario gives an 80\% power (alpha $=0.05$ ).

b. Data analysis

To test differences between two subgroups on the course of QoL since baseline, we will carry out a mixed model analysis to accommodate for hospital effects and repeated measurements. All secondary outcome measures will be analyzed using mixed models in a similar manner. Cost-effectiveness, as far as it concerns the empirical data, is analyzed in a stochastic fashion using bootstrapped regression based techniques (i.e. linear mixed model) adhering to the net benefit framework.

c. Safety

An independent Data Safety Monitoring Board (DSMB) is established, existing of three independent experts who have no conflict of interest. This committee will meet once a year to perform interim analysis specifically with respect to safety. The DSMB will report to the study coordinator and may recommend changes in the conduct of the study or even premature study termination.

\section{Ethics}

The study is conducted according to the principles of the Declaration of Helsinki (2008) and to the Medical Research Involving Human Subjects Act (Dutch: WMO). The protocol has been medicalethically approved to be conducted in all 11 centers by the Medical-Ethical Committee of ArnhemNijmegen (NL 50048.091.14). The participating centers are the Radboud university medical center Nijmegen, Maastricht University Medical Centre, Erasmus MC Cancer Clinic Rotterdam, Center for Gynecological Oncology Amsterdam (CGOA): Iocation Netherlands Cancer Institute/Antoni van Leeuwenhoek Hospital and location Amsterdam Medical Center, University Medical Center Groningen, UMC Utrecht Cancer Centre, Leiden University Medical Centre, Gynecologic Oncologic Center South: two locations of Elisabeth-TweeSteden Hospital Tilburg and location Catharina 
Hospital Eindhoven. Furthermore, the protocol is registered in Clinicaltrials.gov (NCT02321228). Written informed consent is obtained from all patients before enrollment.

\section{DIScussion}

In this study protocol, we describe a prospective non-randomized multicenter trial in premenopausal $B R C A$ mutation carriers. We compare the standard strategy to reduce ovarian cancer risk, i.e. RRSO at recommended age of 35-40 in BRCA1 and at recommended age of 40-45 in BRCA2 mutation carriers, with an innovative risk-reducing strategy. In this innovative strategy, early RRS is performed upon completion of childbearing and subsequent RRO is delayed for five years compared to the currently recommended age for the standard strategy. The primary outcome measure is menopause-related QoL. Secondary outcome measures include safety (cancer incidence and surgical complications), histopathological findings of surgery specimens, cardiovascular risk factors and cost-effectiveness.

Currently, there are two other ongoing studies investigating different aspects of salpingectomy in germline BRCA mutation carriers. A research group from Texas investigates patient compliance with delayed oophorectomy after having undergone prophylactic salpingectomy (NCT01907789). They compare three regimens: ovarian cancer screening (3 years follow-up), prophylactic salpingectomy with delayed oophorectomy (4 years of follow-up including 1 year after oophorectomy) and riskreducing salpingo-oophorectomy (1 year follow-up). QoL is measured as well. Like our study, they do not randomize. This study focuses on another endpoint, i.e. whether BRCA mutation carriers return for oophorectomy after earlier salpingectomy. Duration of follow-up is adjusted to this endpoint en is relatively short to assess the safety of RRS with delayed RRO as it comes to cancer incidence and noncancer-related morbidity. In our study, we focus on QoL, and several subdomains of QoL are measured as well. Nevertheless, our follow-up will not be ceased after QoL data completion, but will be prolonged to guarantee a close monitoring of cancer incidence and noncancer-related morbidity.

In a French study, BRCA mutation carriers who are reluctant to RRSO because of onset of premature menopause are offered a radical fimbriectomy as alternative (NCT01608074). Primary outcome is the number of pelvic serous carcinomas occurring between fimbriectomy and menopause. Secondary outcomes are perioperative morbidity, histopathologic findings of fimbriectomy specimens, incidence of breast cancer and the rate of secondary oophorectomy and associated morbidity. In this study, fimbriectomy is only offered to women who refuse RRSO and RRS will in principle not be followed by RRO, while all women in our study eventually undergo RRO (current uptake of RRSO among BRCA mutation carriers is $95 \%$ in the Netherlands). Furthermore, in this French study BRCA mutation carriers have to be older than 35 years to be included. We include women from 25 years old, to optimize possible risk reduction by removing the fallopian tubes as 
early as possible upon completion of childbearing. At last, the possible advantages of preservation of the ovaries for QoL are not evaluated in this fimbriectomy study, while this is the primary outcome in our study.

In conclusion, the current standard RRSO at age 35-40 (BRCA1) or 40-45 (BRCA2) is highly effective in reducing ovarian cancer incidence. However, consequent premature surgical menopause comes with short- and long-term noncancer-related morbidity and probably affects QoL. New insights in the origin of serous pelvic cancer put the fallopian tube forward as target for alternative preventive surgery. The extent of the role of the fallopian tubes in ovarian carcinogenesis remains uncertain. We expect that early salpingectomy with delayed oophorectomy is a reasonable alternative to preserve ovarian function towards the age of natural menopause without a significant increase in ovarian cancer incidence.

\section{Acknowledgments}

This study is funded by the Dutch Cancer Society, project number KUN 2014-7187. We would like to thank our panel of BRCA mutation carriers who were willing to contribute to this study protocol and associated documents by giving insight in patients' perspectives. 


\section{REFEREMCES}

1. Antoniou A, Pharoah PD, Narod S, et al. Average risks of breast and ovarian cancer associated with BRCA1 or BRCA2 mutations detected in case Series unselected for family history: a combined analysis of 22 studies. Am J Hum Genet 2003; 72(5): 1117-30.

2. Chen S, Parmigiani G. Meta-analysis of BRCA1 and BRCA2 penetrance. J Clin Oncol 2007; 25(11): 1329-33.

3. Vencken PM, Reitsma W, Kriege M, et al. Outcome of BRCA1- compared with BRCA2-associated ovarian cancer: a nationwide study in the Netherlands. Ann Oncol 2013; 24(8): 2036-42.

4. Russo A, Calo V, Bruno L, Rizzo S, Bazan V, Di Fede G. Hereditary ovarian cancer. Crit Rev Oncol Hematol 2009; 69(1): 28-44.

5. Bolton KL, Chenevix-Trench G, Goh C, et al. Association between BRCA1 and BRCA2 mutations and survival in women with invasive epithelial ovarian cancer. JAMA : the journal of the American Medical Association 2012; 307(4): 382-90.

6. Mavaddat N, Barrowdale D, Andrulis IL, et al. Pathology of breast and ovarian cancers among BRCA1 and BRCA2 mutation carriers: results from the Consortium of Investigators of Modifiers of BRCA1/2 (CIMBA). Cancer Epidemiol Biomarkers Prev 2012; 21 (1): 134-47.

7. Vasen HF, Tesfay E, Boonstra $\mathrm{H}$, et al. Early detection of breast and ovarian cancer in families with BRCA mutations. Eur J Cancer 2005; 41 (4): 549-54.

8. Oei AL, Massuger LF, Bulten J, Ligtenberg MJ, Hoogerbrugge N, de Hullu JA. Surveillance of women at high risk for hereditary ovarian cancer is inefficient. Br J Cancer 2006; 94(6): 814-9.

9. Hermsen BB, Olivier RI, Verheijen RH, et al. No efficacy of annual gynaecological screening in BRCA1/2 mutation carriers; an observational follow-up study. Br J Cancer 2007; 96(9): 1335-42.

10. Domchek SM, Friebel TM, Singer CF, et al. Association of risk-reducing surgery in BRCA1 or BRCA2 mutation carriers with cancer risk and mortality. JAMA : the journal of the American Medical Association 2010; 304(9): 967-75.

11. Rebbeck TR, Kauff ND, Domchek SM. Meta-analysis of risk reduction estimates associated with riskreducing salpingo-oophorectomy in BRCA1 or BRCA2 mutation carriers. J Nat/ Cancer Inst 2009; 101 (2): 80-7.

12. Kauff ND, Domchek SM, Friebel TM, et al. Risk-reducing salpingo-oophorectomy for the prevention of BRCA1- and BRCA2-associated breast and gynecologic cancer: a multicenter, prospective study. J Clin Oncol 2008; 26(8): 1331-7.

13. Richtlijn Hereditair Mamma/Ovariumcarcinoom. 2010-07-01 2012. http://www.oncoline.n//hereditairmamma-ovariumcarcinoom (accessed 26th February 2014).

14. Finch A, Beiner M, Lubinski J, et al. Salpingo-oophorectomy and the risk of ovarian, fallopian tube, and peritoneal cancers in women with a BRCA1 or BRCA2 Mutation. JAMA : the journal of the American Medical Association 2006; 296(2): 185-92.

15. Rebbeck TR, Lynch HT, Neuhausen SL, et al. Prophylactic oophorectomy in carriers of BRCA1 or BRCA2 mutations. N Engl J Med 2002; 346(21): 1616-22.

16. Finch AP, Lubinski J, Moller $\mathrm{P}$, et al. Impact of oophorectomy on cancer incidence and mortality in women with a BRCA1 or BRCA2 mutation. J Clin Oncol 2014; 32(15): 1547-53.

17. Kauff ND, Satagopan JM, Robson ME, et al. Risk-reducing salpingo-oophorectomy in women with a BRCA1 or BRCA2 mutation. NEng/ J Med 2002; 346(21): 1609-15.

18. Kenkhuis MJ, de Bock GH, Elferink PO, et al. Short-term surgical outcome and safety of risk reducing salpingo-oophorectomy in BRCA1/2 mutation carriers. Maturitas 2010; 66(3): 310-4.

19. Dowdy SC, Stefanek M, Hartmann LC. Surgical risk reduction: prophylactic salpingo-oophorectomy and prophylactic mastectomy. Am J Obstet Gynecol 2004; 191(4): 1113-23.

20. Shuster LT, Gostout BS, Grossardt BR, Rocca WA. Prophylactic oophorectomy in premenopausal women and long-term health. Menopause Int 2008; 14(3): 111-6. 
21. Svejme O, Ahlborg HG, Nilsson JA, Karlsson MK. Early menopause and risk of osteoporosis, fracture and mortality: a 34-year prospective observational study in 390 women. BJOG 2012; 119(7): 810-6.

22. Arts-de Jong M, Maas AH, Massuger LF, Hoogerbrugge N, de Hullu JA. BRCA1/2 mutation carriers are potentially at higher cardiovascular risk. Crit Rev Oncol Hematol 2014.

23. Madalinska JB, van Beurden M, Bleiker EM, et al. The impact of hormone replacement therapy on menopausal symptoms in younger high-risk women after prophylactic salpingo-oophorectomy. J Clin Oncol 2006; 24(22): 3576-82.

24. Fakkert IE, Mourits MJ, Jansen L, et al. Breast Cancer Incidence After Risk-Reducing SalpingoOophorectomy in BRCA1 and BRCA2 Mutation Carriers. Cancer Prev Res (Phila) 2012; 5(11): 1291-7.

25. Mavaddat N, Peock S, Frost D, et al. Cancer risks for BRCA1 and BRCA2 mutation carriers: results from prospective analysis of EMBRACE. J Natl Cancer Inst 2013; 105(11): 812-22.

26. Heemskerk-Gerritsen BA, Seynaeve C, van Asperen CJ, et al. Breast cancer risk after salpingooophorectomy in healthy BRCA1/2 mutation carriers: revisiting the evidence for risk reduction. J Natl Cancer Inst 2015; 107(5).

27. Dubeau L, Drapkin R. Coming into focus: the nonovarian origins of ovarian cancer. Ann Oncol 2013; 24 Suppl 8: viii28-viii35.

28. Collins IM, Domchek SM, Huntsman DG, Mitchell G. The tubal hypothesis of ovarian cancer: caution needed. Lancet Oncol 2011; 12(12): 1089-91.

29. Erickson BK, Conner MG, Landen CN, Jr. The role of the fallopian tube in the origin of ovarian cancer. Am J Obstet Gynecol 2013; 209(5): 409-14.

30. Dietl J. Revisiting the pathogenesis of ovarian cancer: the central role of the fallopian tube. Arch Gynecol Obstet 2014; 289(2): 241-6.

31. Piek JM, van Diest PJ, Zweemer RP, et al. Dysplastic changes in prophylactically removed Fallopian tubes of women predisposed to developing ovarian cancer. J Pathol 2001; 195(4): 451-6.

32. Piek JM, Verheijen RH, Kenemans P, Massuger LF, Bulten H, van Diest PJ. BRCA1/2-related ovarian cancers are of tubal origin: a hypothesis. Gynecol Oncol 2003; 90(2): 491.

33. Roh MH, Kindelberger D, Crum CP. Serous tubal intraepithelial carcinoma and the dominant ovarian mass: clues to serous tumor origin? Am J Surg Pathol 2009; 33(3): 376-83.

34. Kindelberger DW, Lee $Y$, Miron A, et al. Intraepithelial carcinoma of the fimbria and pelvic serous carcinoma: Evidence for a causal relationship. Am J Surg Pathol 2007; 31(2): 161-9.

35. Przybycin CG, Kurman RJ, Ronnett BM, Shih le M, Vang R. Are all pelvic (nonuterine) serous carcinomas of tubal origin? Am J Surg Pathol 2010; 34(10): 1407-16.

36. Kuhn E, Kurman RJ, Vang R, et al. TP53 mutations in serous tubal intraepithelial carcinoma and concurrent pelvic high-grade serous carcinoma--evidence supporting the clonal relationship of the two lesions. J Pathol 2012; 226(3): 421-6.

37. Tone AA, Begley H, Sharma M, et al. Gene expression profiles of luteal phase fallopian tube epithelium from BRCA mutation carriers resemble high-grade serous carcinoma. Clin Cancer Res 2008; 14(13): 406778.

38. Carcangiu ML, Radice P, Manoukian S, et al. Atypical epithelial proliferation in fallopian tubes in prophylactic salpingo-oophorectomy specimens from BRCA1 and BRCA2 germline mutation carriers. Int J Gynecol Pathol 2004; 23(1): 35-40.

39. Callahan MJ, Crum CP, Medeiros F, et al. Primary fallopian tube malignancies in BRCA-positive women undergoing surgery for ovarian cancer risk reduction. J Clin Oncol 2007; 25(25): 3985-90.

40. Rabban JT, Barnes M, Chen LM, Powell CB, Crawford B, Zaloudek CJ. Ovarian pathology in risk-reducing salpingo-oophorectomies from women with BRCA mutations, emphasizing the differential diagnosis of occult primary and metastatic carcinoma. Am J Surg Pathol 2009; 33(8): 1125-36.

41. Shaw PA, Rouzbahman M, Pizer ES, Pintilie M, Begley H. Candidate serous cancer precursors in fallopian tube epithelium of BRCA1/2 mutation carriers. Mod Pathol 2009; 22 (9): 1133-8. 
42. Manchanda R, Abdelraheim A, Johnson M, et al. Outcome of risk-reducing salpingo-oophorectomy in BRCA carriers and women of unknown mutation status. BJOG 2011; 118(7): 814-24.

43. Leonhardt K, Einenkel J, Sohr S, Engeland K, Horn LC. p53 signature and serous tubal in-situ carcinoma in cases of primary tubal and peritoneal carcinomas and serous borderline tumors of the ovary. Int $J$ Gynecol Pathol 2011; 30(5): 417-24.

44. Mingels MJ, Roelofsen T, van der Laak JA, et al. Tubal epithelial lesions in salpingo-oophorectomy specimens of BRCA-mutation carriers and controls. Gynecol Oncol 2012; 127(1): 88-93.

45. Reitsma W, Mourits MJ, de Bock GH, Hollema H. Endometrium is not the primary site of origin of pelvic high-grade serous carcinoma in BRCA1 or BRCA2 mutation carriers. Mod Pathol 2013; 26(4): 572-8.

46. Wethington SL, Park KJ, Soslow RA, et al. Clinical outcome of isolated serous tubal intraepithelial carcinomas (STIC). Int J Gynecol Cancer 2013; 23(9): 1603-11.

47. Cass I, Walts AE, Barbuto D, Lester J, Karlan B. A cautious view of putative precursors of serous carcinomas in the fallopian tubes of BRCA mutation carriers. Gynecol Oncol 2014; 134(3): 492-7.

48. Greene MH, Mai PL, Schwartz PE. Does bilateral salpingectomy with ovarian retention warrant consideration as a temporary bridge to risk-reducing bilateral oophorectomy in BRCA1/2 mutation carriers? Am J Obstet Gynecol 2011; 204(1): 19 e1-6.

49. Kwon JS, Tinker A, Pansegrau G, et al. Prophylactic salpingectomy and delayed oophorectomy as an alternative for BRCA mutation carriers. Obstet Gynecol 2013; 121(1): 14-24.

50. Holman LL, Friedman S, Daniels MS, Sun CC, Lu KH. Acceptability of prophylactic salpingectomy with delayed oophorectomy as risk-reducing surgery among BRCA mutation carriers. Gynecol Oncol 2014; 133(2): 283-6.

51. Arts-de Jong M, Harmsen MG, Hoogerbrugge N, Massuger LF, Hermens RP, de Hullu JA. Risk-reducing salpingectomy with delayed oophorectomy in BRCA1/2 mutation carriers: patients' and professionals' perspectives. Gynecol Oncol 2015; 136(2): 305-10.

52. Greene JG. Constructing a standard climacteric scale. Maturitas 1998; 29(1): 25-31.

53. Leblanc E, Narducci F, Farre I, et al. Radical fimbriectomy: a reasonable temporary risk-reducing surgery for selected women with a germ line mutation of BRCA 1 or 2 genes? Rationale and preliminary development. Gynecol Oncol 2011; 121(3): 472-6.

54. Ware JE, Jr., Sherbourne CD. The MOS 36-item short-form health survey (SF-36). I. Conceptual framework and item selection. Med Care 1992; 30(6): 473-83.

55. EuroQol G. EuroQol--a new facility for the measurement of health-related quality of life. Health Policy (New York) 1990; 16(3): 199-208.

56. Lerman C, Daly M, Masny A, Balshem A. Attitudes about genetic testing for breast-ovarian cancer susceptibility. J Clin Onco/ 1994; 12(4): 843-50.

57. Watson M, Duvivier V, Wade Walsh M, et al. Family history of breast cancer: what do women understand and recall about their genetic risk? J Med Genet 1998; 35(9): 731-8.

58. Rosen R, Brown C, Heiman J, et al. The Female Sexual Function Index (FSFI): a multidimensional selfreport instrument for the assessment of female sexual function. J Sex Marital Ther 2000; 26(2): 191-208.

59. ter Kuile MM, Brauer M, Laan E. The Female Sexual Function Index (FSFI) and the Female Sexual Distress Scale (FSDS): psychometric properties within a Dutch population. J Sex Marital Ther 2006; 32 (4): 289-304.

60. Derogatis LR, Rosen R, Leiblum S, Burnett A, Heiman J. The Female Sexual Distress Scale (FSDS): initial validation of a standardized scale for assessment of sexually related personal distress in women. J Sex Marital Ther 2002; 28 (4): 317-30.

61. O'Connor AM. Validation of a decisional conflict scale. Med Decis Making 1995; 15(1): 25-30.

62. Bouwmans C, Hakkaart-van Roijen L, Koopmanschap MA, Krol M, Severens H, Brouwer W. Manual of the iMTA Productivity Cost Questionnaire (iPCQ). Rotterdam: iMTA, Erasmus University Rotterdam; 2013.

63. Bouwmans C, Hakkaart-van Roijen L, Koopmanschap MA, Krol M, Severens H, Brouwer W. Handleiding iMTA Medical Cost Questionnaire (iMCQ). Rotterdam: iMTA, Erasmus University; 2013. 
64. Crum CP, Drapkin R, Miron A, et al. The distal fallopian tube: a new model for pelvic serous carcinogenesis. Curr Opin Obstet Gynecol 2007; 19(1): 3-9.

65. Brehaut JC, O'Connor AM, Wood TJ, et al. Validation of a decision regret scale. Med Decis Making 2003; 23(4): 281-92.

66. Meeuwissen PA, Seynaeve C, Brekelmans CT, Meijers-Heijboer HJ, Klijn JG, Burger CW. Outcome of surveillance and prophylactic salpingo-oophorectomy in asymptomatic women at high risk for ovarian cancer. Gynecol Oncol 2005; 97 (2): 476-82.

67. Barentsen R, van de Weijer PH, van Gend S, Foekema H. Climacteric symptoms in a representative Dutch population sample as measured with the Greene Climacteric Scale. Maturitas 2001; 38(2): 123-8. 

$\therefore$

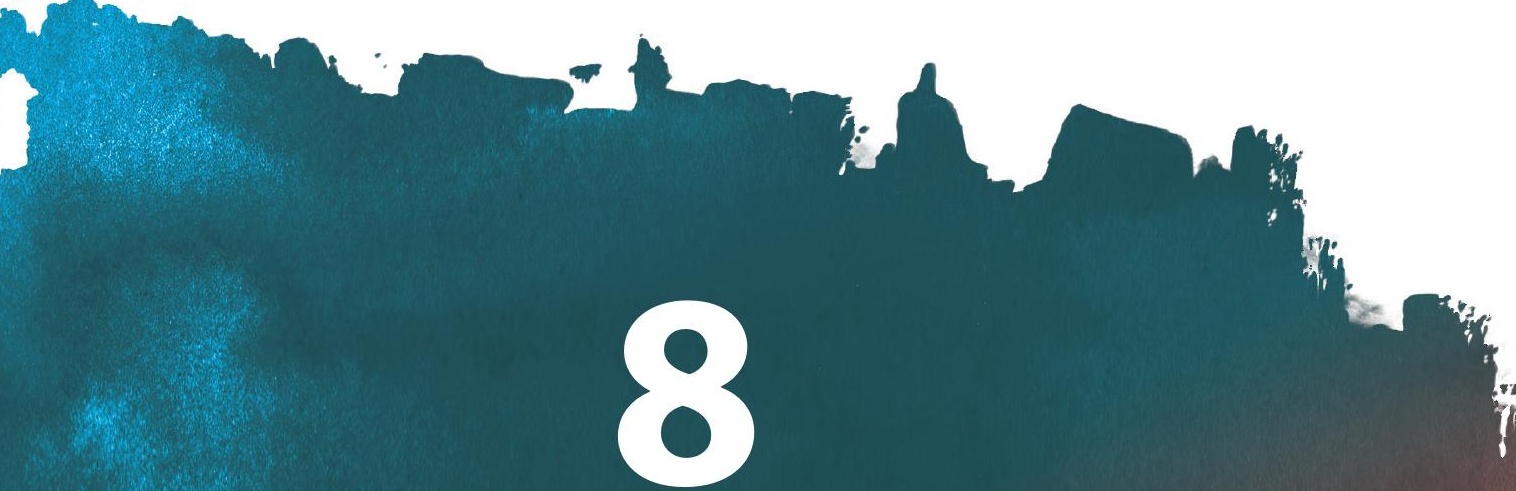

\section{A patient decision aid for risk-}

reducing surgery in premenopausal

\section{BRCA1/2 mutation carriens:}

development process and pilot testing

Marline G. Harmsen, Nicoline Hoogerbrugge, Helena C. van Doorn,

Katja N. Gaarenstroom, M. Caroline Vos, Leon F.A.G. Massuger, Joanne A. de Hullu,

Rosella P.M.G. Hermens 


\section{ABSTRACT}

\section{Objective}

Risk-reducing salpingo-oophorectomy around 40 years of age is currently recommended to $B R C A 1 / 2$ mutation carriers to reduce their increased ovarian cancer risk. Premature menopause is the main adverse effect of this procedure. Therefore, an alternative strategy consisting of salpingectomy with delayed oophorectomy is currently being investigated in a preference trial. A patient decision aid that combines evidence with patient preferences is needed to facilitate this complex decision-making process.

\section{Methods}

A patient decision aid was systematically developed in an iterative process of prototype development, pilot testing by patients and clinicians, and revisions. Information was based on available literature and current guidelines. Development was in accordance with the International Patient Decision Aid Standards (IPDAS) quality criteria. A multidisciplinary steering group supervised the process.

\section{Results}

The patient decision aid underwent four rounds of pilot testing and revisions. Finally, two paper decision aids were developed: one for BRCA1, one for BRCA2. They both contained a general introduction, three chapters, and a step-by-step plan containing a personal value clarification worksheet. During pilot testing, risk communication and information about premature menopause and hormone replacement therapy were the most revised items. The patient decision aids fulfill 37 of 43 IPDAS criteria for content and development process. Effectiveness has not been evaluated yet.

\section{Conclusion}

Both BRCA1/2 mutation carriers and professionals are willing to use or offer the developed patient decision aids for the choice between risk-reducing salpingo-oophorectomy and salpingectomy with delayed oophorectomy. The patient decision aids have been found clear, balanced and comprehensible. Future testing among patients facing the decision should point out its effectiveness in improving decision-making. 


\section{InTRODUCTION}

Women harboring a germline mutation in the BRCA1 and/or BRCA2 genes have an increased risk of breast and ovarian cancer.' The most effective way to diminish this ovarian cancer risk is removing fallopian tubes and ovaries during risk-reducing salpingo-oophorectomy, preferably around the age of 40 years. 2,3 However, salpingo-oophorectomy at that age results in acute onset of premature menopause with several short- and long-term health consequences. ${ }^{4-6}$

Therefore, salpingectomy upon completion of childbearing with delayed oophorectomy has been proposed as alternative strategy to reduce ovarian cancer risk. ${ }^{7-9}$ This strategy is based on the growing evidence that (serous) ovarian cancer mainly originates from the fallopian tube, ${ }^{10-12}$ and is currently being investigated in a Dutch preference trial (NCT02321228). ${ }^{13}$ However, safety in terms of ovarian cancer risk has not been proven yet, which is the main disadvantage of this alternative strategy.

Because of different pros and cons of both strategies, the choice of trial participants between risk-reducing salpingo-oophorectomy and salpingectomy with delayed oophorectomy is complex and highly personal. It is important to educate and empower these women in the decision-making process. Previously, patient decision aids have been shown to increase knowledge, improve risk perception, lower decisional conflict, reduce proportions of people remaining undecided, and can improve patient satisfaction. ${ }^{14}$ Interestingly, they also increase the number of patients who prefer conservative treatment options rather than invasive surgery. ${ }^{14}$

Our aim was to systematically develop and pilot-test a patient decision aid for ovarian cancer risk reduction in BRCA1/2 mutation carriers that combines evidence with patient preferences.

\section{METHODS}

\section{Development}

Recommendations published by Coulter et al. ${ }^{15}$ guided the development process of the patient decision aid (Figure 1). The prototype was developed by a project group consisting of a medical doctor, a gynecologic oncologist, and an expert in shared decision-making and guideline implementation, assisted by a patient and professional expert panel, and in accordance with the international quality criteria of the International Patient Decision Aid Standards (IPDAS). ${ }^{16}$ The development process was supervised by a steering group, consisting of a professor of gynecologic oncology, professor of hereditary cancer, and biostatistician. None of the steering group members had any conflicts of interest. 


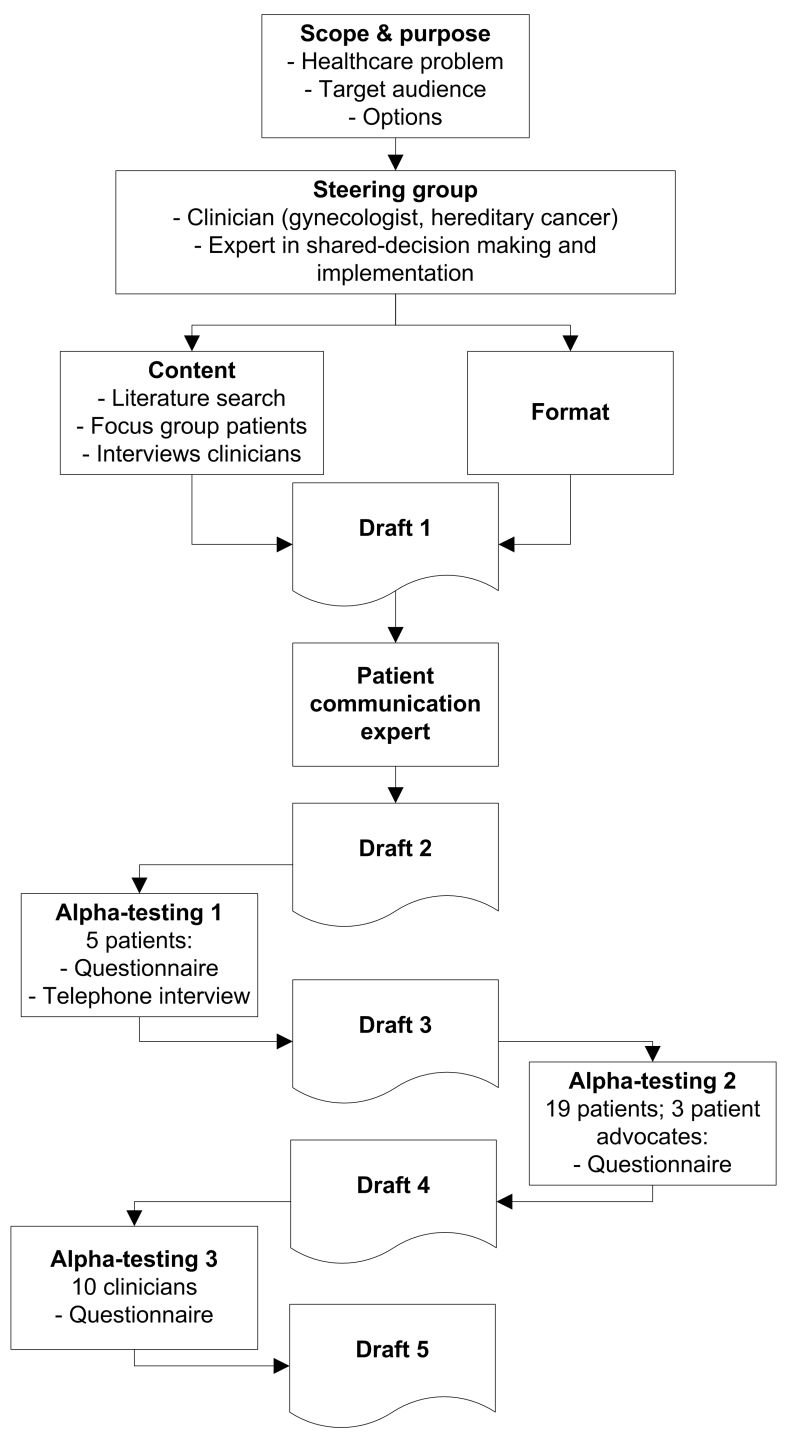

Figure 1. Systematic development process of the patient decision aid

\section{Scope and purpose}

Given the complex decision that has to be made within our preference trial on risk-reducing salpingo-oophorectomy and salpingectomy with delayed oophorectomy in BRCA1/2 mutation carriers (NCT02321228), ${ }^{13}$ the project and steering group agreed on the need for a decision support tool for trial participants. Options to be discussed in the decision aid resulted from the two possible options in the preference trial. Besides, watchful waiting (i.e., no risk-reducing surgery) completed the overview of options. 


\section{Exploring patients' decisional needs}

Points of consideration in the choice between risk-reducing salpingo-oophorectomy and salpingectomy with delayed oophorectomy were extracted from literature, searching the Medline database for "BRCA" or equivalents and "salpingectomy" or "delayed oophorectomy". Furthermore, we interviewed BRCA1/2 mutation carriers and their healthcare professionals in the process of designing the aforementioned preference trial. ${ }^{8}$ The purpose of these interviews was to explore barriers and facilitators for the introduction of salpingectomy with delayed oophorectomy as alternative risk-reducing strategy. One year later, a random selection of these women attended another session to discuss the content of the trial patient information booklet and decision aid.

\section{Content and format}

Based on the identified decisional needs, guidelines, and expert opinions, the project group together with the steering group determined five domains that are important for decisionmaking in this setting: 1) risks and 2) benefits of watchful waiting, salpingo-oophorectomy, and salpingectomy with delayed oophorectomy; 3) premature menopause and hormone replacement therapy (HRT); 4) strength and availability of evidence; 5) women's preferences. For the first three domains, we collected available literature to compose the content of the decision aid; the fourth domain was based on appraisal of the evidence found. The literature search for domains 1) and 2) is described in detail elsewhere. ${ }^{17}$ The Medline database was searched for the third domain using the terms "BRCA" or equivalents and "menopause" or equivalents in September 2014. For the fifth domain, the best way to clarify individual preferences was also extracted from literature and combined with expert opinions within our project group. To be able to hand out and discuss the patient decision aid at the outpatient department, the patient decision aid was developed as print booklet that can be used complimentary to face-to-face counseling. The booklet is also a good format to be mailed.

\section{Pilot testing and revision}

An iterative process of reviewing and revising was followed to develop the version of the patient decision aid that is ready for field testing. The first draft was evaluated by a patient communication expert to ameliorate comprehensibility.

The second draft was sent to a patient expert panel consisting of six BRCA1/2 mutation carriers who had one or more consultations at our tertiary hospital and had already undergone risk-reducing salpingo-oophorectomy. Although they do not belong to the ultimate target population, we invited these women to assess the decision aid for two reasons: first, they know the consequences of salpingo-oophorectomy from their own experience and second, we considered it unethical to offer a preliminary version of the decision aid to women who still had to make a choice for either strategy. They were asked to fill out a questionnaire on structure, content, layout, length, comprehensibility, relevance, credibility and usability of the patient decision aid that was (e)mailed 
to them. The questionnaire contained 21 questions, of which 13 were open-ended, and was previously used in the development of another patient decision aid. ${ }^{18}$ Additionally, the decision aid was discussed during an individual audio-recorded telephone interview to let them 'think aloud'. Based on the received feedback, a third draft was developed and pilot-tested. Forty-four BRCA1/2 mutation carriers who had undergone premenopausal risk-reducing salpingo-oophorectomy in our hospital between 2010 and 2015 were sent an invitation letter and informed consent form. A reminder was sent after one month in case of no response. Women who consented to participate were sent the decision aid and a short questionnaire with 12 Likert scale questions, 12 multiplechoice items and two opportunities for suggestions. Questions were about basic demographics, content, structure, length, balance, comprehensibility, completeness, and usefulness. The main difference with the questionnaire in the previous test round was a lower number of open-ended questions. Again, the questionnaire was based on a questionnaire previously used. ${ }^{18}$ Participants who did not return their questionnaires were repeatedly reminded. Furthermore, feedback on the decision aid was also requested from patient advocates. Based on feedback on the third draft, a revised version together with an adjusted questionnaire was emailed to an expert panel of 14 healthcare professionals from ten hospitals throughout the country who counsel participants about ovarian cancer risk-reducing options in the national preference trial. They were not involved in the initial development of the decision aid. Their suggestions were incorporated in the final version that is ready for field testing.

Based on the study design and confirmed by the Medical Ethical Committee 'CMO Regio ArnhemNijmegen', the Medical Research Involving Human Subjects Act (Dutch: WMO) is not applicable to this study. Therefore, the study was exempted from being appraised by a medical ethical committee.

\section{RESULTS}

\section{Development}

Scope and purpose

The purpose of the patient decision aid is to provide decision support for BRCA1/2 mutation carriers who participate in the preference trial that compares risk-reducing salpingo-oophorectomy with salpingectomy and delayed oophorectomy. Therefore, the target audience consists of all trial participants: premenopausal BRCA1/2 mutation carriers who completed childbearing, who are 2540 (BRCA1) or 25-45 (BRCA2) years old, and who are currently not being treated for any malignancy. Three options are discussed in the decision aid. The first option is watchful waiting: no risk-reducing surgery. The second option is risk-reducing salpingo-oophorectomy between 35-40 years old (BRCA1) or 40-45 years old (BRCA2), as currently recommended in national and international guidelines. The third option is risk-reducing salpingectomy upon completion of childbearing with 
delayed oophorectomy between 40-45 (BRCA1) or 45-50 years old (BRCA2), which has not been proven effective yet in terms of improved quality of life or safe in terms of ovarian cancer risk. To stick closely to the objective of the preference trial and to limit the length of the decision aid, the project group decided to focus on ovarian cancer risk management. Hence, information on breast surveillance and risk-reducing mastectomy was not included. However, we did discuss the effect of the three aforementioned options on breast cancer risk.

\section{Patients' decisional needs}

From our qualitative study, we identified the following factors important in the decision for salpingo-oophorectomy or salpingectomy with delayed oophorectomy: ovarian and breast cancer risks; onset of premature menopause; level of evidence for the efficacy and safety of either strategy; medical history; family history; number of operations. ${ }^{8}$ The additional literature search yielded two publications on BRCA1/2 mutation carriers ${ }^{9}$ and professionals ${ }^{19}$ surveyed about salpingectomy with delayed oophorectomy. Factors reported to influence the decision are consequences of premature menopause, HRT, surgical morbidity, potential ovarian damage, and lack of data on level of benefit and cancer risks. ${ }^{9,19}$

\section{Content and format}

Search results for domains 1) risks and 2) benefits of watchful waiting, salpingo-oophorectomy, and salpingectomy with delayed oophorectomy are described elsewhere. ${ }^{17}$ Risk estimates based on literature were incorporated into the decision aid. Literature search for the third domain concerning premature menopause and HRT yielded 79 publications. Two relevant articles outlining all aspects of premature menopause and HRT in BRCA1/2 mutation carriers were identified and used in the decision aid. ${ }^{20,21}$ Critical appraisal of the level of evidence of selected publications (fourth domain) resulted in how conclusively information was presented. The format of the value clarification worksheet (fifth domain) was based on a systematic review ${ }^{22}$ and the experience within our project group. 18,23

Analogous to another decision aid, ${ }^{23}$ ours was subdivided into a general introduction, three chapters, and a step-by-step plan containing a personal value clarification worksheet. Chapter 1 outlined the three options in ovarian cancer risk management with the main risks and benefits. Chapter 2 contained more specific information on (estimated) ovarian and breast cancer risks. Chapter 3 goes into menopause and HRT. The step-by-step plan contained 9 steps to guide the user which information should be read, which individual values matter most, and whether one is well-prepared and confident enough to decide. To elicit one's preferences, eight statements were selected based on patients' decisional needs and had to be scored for (dis)agreement on a 6-point Likert scale (3-point Likert scale in the initial version). Subsequently, the user is asked to rank the three most important statements to clarify which values matter most; however, first drafts contained a rating instead of a ranking exercise. Finally, implications of their stated values for their decision are explicitly shown. 


\section{Pilot testing and revision}

Pilot 1

After the text of the first draft was adapted according to the patient communication expert's comments, 5 of 6 invited BRCA1/2 mutation carriers consented to participate in pilot test 1. They filled out a questionnaire and audio-recorded telephone interviews were subsequently transcribed by one of the authors (M.H.). Their comments led to several major changes. An image of the anatomy of the female internal genitals was added, as was an overview of options with their main (dis)advantages. Participants preferred icon arrays and pie charts for risk communication so risk tables, that were found confusing, were deleted. Limited alterations were made in wording, text order, and color use which resulted in the third draft.

\section{Pilot 2}

Twenty-five of 44 eligible women (57\%) signed and returned the informed consent form for participation in the next pilot, two declined and 17 responded neither to our letter nor to a reminder. Nineteen of 25 women (76\%) who consented filled out the semi-quantitative questionnaire. Four did not return their questionnaires in spite of several reminders, one withdrew her consent, and one was physically not able to complete the questionnaire. Baseline characteristics of the 19 participants are presented in Table 1. Mean age at risk-reducing salpingo-oophorectomy was 42.3 years and 14 (73.7\%) carried a BRCA1 mutation. Twenty percent were breast cancer survivors and $42 \%$ were higher educated.

Table 1. Baseline characteristics of BRCA1/2 mutation carriers who had already undergone risk-reducing salpingo-oophorectomy and participated in Pilot 2

\begin{tabular}{|c|c|c|}
\hline & $N=19$ & \\
\hline Current age (years, mean \pm SD) & $45.6 \pm 3.878$ & range $37-51$ \\
\hline Age at RRSO (years, mean \pm SD) & $42.3 \pm 3.603$ & range $34-48$ \\
\hline \multicolumn{3}{|l|}{ Mutation (n, \%) } \\
\hline$B R C A 1$ & 14 & 73.7 \\
\hline$B R C A 2$ & 5 & 26.3 \\
\hline \multicolumn{3}{|l|}{ Previous breast cancer (n, \%) } \\
\hline Yes & 4 & 21.1 \\
\hline No & 15 & 78.9 \\
\hline \multicolumn{3}{|l|}{ Level of education (n, \%) } \\
\hline Primary/pre-vocational school & 2 & 10.5 \\
\hline Vocational education & 7 & 36.9 \\
\hline Pre-college education & 2 & 10.5 \\
\hline College/university & 8 & 42.1 \\
\hline
\end{tabular}

Abbreviations: RRSO Risk-reducing salpingo-oophorectomy; 
For chapters 1 and 2 about the three options and (estimated) ovarian and breast cancer risks, the content was judged 'good' or 'excellent' on a 4-point Likert scale by at least $95 \%$ and presentation of information was judged 'good' or 'excellent' by at least $89 \%$. Both content and presentation of information in chapter 3 were scored 'good' or 'excellent' in 84\% (menopause part) and 74\% (HRT part). Results of other items surveyed are presented in Table 2. Length and the amount of information were assessed 'just right' by the majority of participants. Including more information about menopause and about risks and prognosis of ovarian cancer was suggested. Furthermore, the information was mainly judged as balanced, realistic and comprehensible, and 79\% would find it useful in decision-making if they would have to choose between salpingo-oophorectomy and salpingectomy with delayed oophorectomy. The preference elicitation tool of the personal worksheet was the most criticized: $32 \%$ either wanted to add, remove or change some of the statements. No differences were observed between women with and without breast cancer history. In general, patient reviewers were very positive about the clear language and images. The decision aid provided readers with easy-to-read information to get an overview of all relevant matters. In contrast, suggestions for improvement were mainly related to information on menopause and HRT, and statements in the preference elicitation tool. A revised version of the decision aid was based on all comments of these 19 patients and four patient advocates. In addition to some textual changes, major changes included revision of chapter 3 (menopause and HRT), adjustment of statement order in the personal worksheet, rephrasing five statements, and replacing one statement. Furthermore, the initial rating exercise was replaced by the ranking exercise at this stage. Moreover, we added more detailed information about ovarian cancer prognosis and about the (estimated) risk reduction by the two strategies to chapter 1.

\section{Pilot 3: professionals}

Ten of 14 (71\%) professionals from eight hospitals then reviewed the decision aid and filled out the questionnaire: eight gynecologic oncologists, one gynecologist, and one medical doctor involved in counseling and supportive care of BRCA1/2 mutation carriers. For chapters 1 and 2 about the three options and (estimated) cancer risks, both content and presentations were judged 'good' or 'excellent' on a 4-point Likert scale by at least 70\% of professionals, except for the presentation of HRT that was scored 'good' or 'excellent' by $60 \%$ and 'moderate' by the remaining $40 \%$. The majority (70\%) found the decision aid too long. Five suggested to develop two separate booklets for BRCA1 and BRCA2. Another concern for half of the responders was the lack of comprehensibility of the presented risks. However, the vast majority classified the information as balanced and realistic, estimated that it would be a useful tool for BRCA1/2 mutation carriers, and confirmed to be willing to offer this decision aid to their patients additionally to in-person counseling (Table 2). 
Table 2. Pilot testing among BRCA1/2 mutation carriers and their healthcare providers

\begin{tabular}{|c|c|c|}
\hline & $\begin{array}{l}\text { Draft } 3 \\
\text { BRCA mutation carriers } \\
(\mathrm{n}=19)\end{array}$ & $\begin{array}{l}\text { Draft } 4 \\
\text { Healthcare providers } \\
(n=10)\end{array}$ \\
\hline \multicolumn{3}{|l|}{ Length of PtDA (n (\%)) } \\
\hline Too long & $1(5 \%)$ & $7(70 \%)$ \\
\hline Too short & $2(11 \%)$ & 0 \\
\hline Just right & $16(84 \%$ & $2(20 \%)$ \\
\hline Missing & 0 & $1(10 \%)$ \\
\hline \multicolumn{3}{|l|}{ Amount of information ( $\mathrm{n}(\%))$} \\
\hline Too much & $1(5 \%)$ & $1(10 \%)$ \\
\hline Too little & $6(32 \%)$ & $1(10 \%)$ \\
\hline Just right & $12(63 \%)$ & $3(30 \%)$ \\
\hline Missing & 0 & $5(50 \%)$ \\
\hline \multicolumn{3}{|l|}{ Information balanced? (n (\%)) } \\
\hline Yes & $15(79 \%)$ & $8(80 \%)$ \\
\hline Slanted towards RRSO & $3(16 \%)$ & 0 \\
\hline Slanted towards RRS/RRO & $1(5 \%)$ & $2(20 \%)$ \\
\hline \multicolumn{3}{|l|}{ PtDA is comprehensible (n (\%)) } \\
\hline In general & $17(89 \%)$ & $\mathrm{N} / \mathrm{A}$ \\
\hline \multicolumn{3}{|l|}{ Risk communication } \\
\hline Yes & $17(89 \%)$ & $4(40 \%)$ \\
\hline No & $2(11 \%)$ & $4(40 \%)$ \\
\hline Missing & 0 & $2(20 \%)$ \\
\hline \multicolumn{3}{|l|}{ Consequences of RRSO (n (\%)) } \\
\hline Realistic & $16(84 \%)$ & $9(90 \%)$ \\
\hline Underestimated & $3(16 \%)$ & $1(10 \%)$ \\
\hline \multicolumn{3}{|l|}{ Consequences of RRS/RRO (n (\%)) } \\
\hline Realistic & $13(68 \%)$ & $10(100 \%)$ \\
\hline Underestimated & $5(27 \%)$ & 0 \\
\hline Overestimated & $1(5 \%)$ & 0 \\
\hline \multicolumn{3}{|l|}{ Useful in decision-making (n (\%)) } \\
\hline Yes & $15(79 \%)$ & $8(80 \%)$ \\
\hline No & $3(16 \%)$ & 0 \\
\hline Missing & $1(5 \%)$ & $2(20 \%)$ \\
\hline \multicolumn{3}{|l|}{ Sufficient information to decide (n (\%)) } \\
\hline Yes & $16(84 \%)$ & $8(80 \%)$ \\
\hline No & $3(16 \%)$ & $1(10 \%)$ \\
\hline Missing & 0 & $1(10 \%)$ \\
\hline \multicolumn{3}{|l|}{ Personal worksheet statements (n (\%)) } \\
\hline Are well chosen & $12(63 \%)$ & $6(60 \%)$ \\
\hline At least one needs to be removed & $5(27 \%)$ & $1(10 \%)$ \\
\hline At least one needs to be added & $1(5 \%)$ & 0 \\
\hline Missing & $1(5 \%)$ & $3(30 \%)$ \\
\hline \multicolumn{3}{|l|}{ Willing to offer PtDA to patients (n (\%)) } \\
\hline Yes & $\mathrm{N} / \mathrm{A}$ & $8(80 \%)$ \\
\hline No, not this PtDA & $\mathrm{N} / \mathrm{A}$ & $1(10 \%)$ \\
\hline No, no PtDA at all & $\mathrm{N} / \mathrm{A}$ & 0 \\
\hline Missing & $N / A$ & $1(10 \%)$ \\
\hline
\end{tabular}

Abbreviations: PtDA Patient decision aid; RRSO Risk-reducing salpingo-oophorectomy; RRS/RRO Risk-reducing salpingectomy with delayed risk-reducing oophorectomy; N/A Not applicable 
Besides some textual revisions, three significant changes were incorporated into the ultimate version of the decision aid. First, we developed separate decision aids for BRCA1 and BRCA2. Second, examples to estimate ovarian cancer risk were more explicitly described. Third, non-hormonal options to alleviate effects of premature menopause were added to chapter 3 . Figure 2 shows the personal value clarification worksheet (translated from Dutch into English).

\section{Value clarification worksheet}

Please indicate to what extent you agree or disagree with the statements below by ticking the applicable box. There are no right or wrong answers, it is all about your own opinion and your own values. You can write additional arguments that are important to you at the blank spaces ("l" and "J").

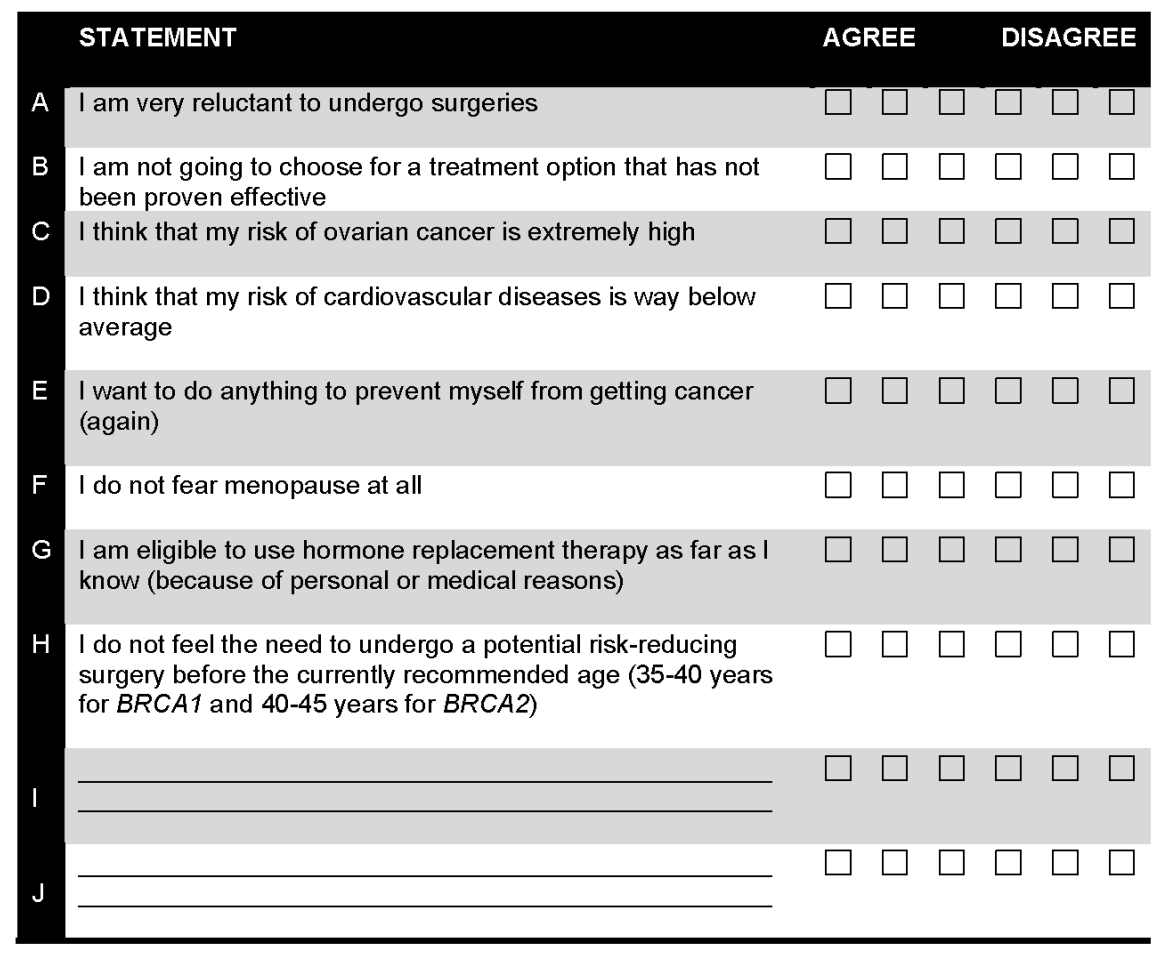

Next, you can rank the three most important statements below by writing down the corresponding letters in the right order (most important statement first).

1.

2.

3.

If you mostly 'agree' with these three statements: risk-reducing salpingo-oophorectomy might be the best choice for you.

If you mostly 'disagree' with these three statements: salpingectomy with delayed oophorectomy might be the best choice for you.

Figure 2. Personal value clarification worksheet of final draft (translated from Dutch into English) 


\section{IPDAS criteria}

Quality of the patient decision aid was tested against the 64 IPDAS criteria. Since none of the additional criteria were applicable, we checked it against the 50'regular' items and 37 were satisfied. Among the 23 criteria for 'Content', only criterion 3.9 was not entirely met: whether the decision aid allows the user to view probabilities based on their own situation (e.g., age). The decision aid provides one example applicable to a certain age. We developed tables with risks for various ages ${ }^{17}$ but those were found to be too complicated for this decision aid. However, they are available for healthcare providers and can be discussed during face-to-face contact. Fifteen of 20 criteria in the 'Development Process' were met. We did not use a readability score to verify readability (criterion 10.4). In addition, we have not field-tested the decision aid yet (criteria 1.4-1.5), although we did pilot-test it. Therefore, criteria regarding the results of field testing were not satisfied: whether it is acceptable (criteria 1.6-1.7), balanced (criterion 9.3), and can be understood by those with limited reading skills (criterion 10.6). Nevertheless, it scored high on the first two criteria during pilot testing. The decision aid was not evaluated by patients with low health literacy, although it was by one dyslectic patient. Lastly, the seven criteria for 'Effectiveness' could not be assessed because we have not field-tested the final version yet.

\section{DIScussIOn}

This paper describes the systematic development process of a paper patient decision aid for ovarian cancer risk management in premenopausal BRCA1/2 mutation carriers, in close collaboration with $B R C A 1 / 2$ mutation carriers and their healthcare professionals. The decision aid elaborates on the risks and benefits of watchful waiting, risk-reducing salpingo-oophorectomy, or salpingectomy with delayed oophorectomy. It can be used additionally to face-to-face consultation. The last version is found to be clear, complete, balanced and usable by the vast majority of reviewers. It fulfills 37 of 43 quality requirements for content and development process as formulated in the IPDAS checklist. Patient reviewers who underwent risk-reducing surgery before, would have liked to use the decision aid if they would have to decide now.

The systematic development in a multidisciplinary team and the input of patient and professional experts are the main strengths of this study. Furthermore, four patient advocates of a national patients' association also reviewed the decision aid. The last strength is that we present the user with the implications of their expressed values in the personal worksheet, supposedly leading to better outcomes when using value clarification methods.22

The most important limitation in the development of this patient decision aid is the lack of (univocal) evidence for some items. First, salpingectomy with delayed oophorectomy has not been clinically investigated yet, so its effect on ovarian cancer risk is unknown. Second, health consequences of premature menopause in this particular population has not been entirely unraveled and the same 
applies to the effect of HRT. However, there are no convincing arguments to expect differences between BRCA1/2 mutation carriers and the general population regarding effects of premature menopause. Furthermore, existing evidence only provides relative risks, which are very hard to be interpreted. For instance, a two- to four-fold increase in the risk of cardiovascular disease is very hard to translate into absolute risks without knowing the background risk, which depends on lifestyle, personal and family medical history etcetera. Furthermore, we did not pilot-test the decision aid in the actual target population because lack of experience with premature menopause and because of ethical reasons. Finally, women with low literacy skills were underrepresented in both pilot tests: a common problem in this kind of research that we were not able to resolve. ${ }^{14}$

Two randomized controlled trials evaluating patient decision aids for women at increased risk of ovarian cancer only included risk-reducing salpingo-oophorectomy and ovarian screening as options. ${ }^{24,25}$ One also contained information on breast cancer risk management options besides those for ovarian cancer. ${ }^{24}$ All together, they found that women who used the decision aid felt better informed and were more satisfied with the amount and quality of received information compared with women who received usual care. Furthermore, users' risk estimates for ovarian cancer were more accurate and they chose risk-reducing surgery more often. However, no statistically significant differences were found for well-being and decision-related outcomes, possibly because of lack of power. Tiller et al..$^{25}$ developed their decision aid for women with a family history of breast and/or ovarian cancer or Lynch syndrome. Besides ovarian screening and risk-reducing salpingo-oophorectomy, information about watchful waiting and chemoprevention was included. Women who used the decision aid reported higher acceptability and the received information as more sufficient and helpful in decision-making compared with women receiving a general educational pamphlet. Greater knowledge and lower decisional conflict were found two weeks after the intervention but did not last after 6 months. Use of the decision aid did not affect psychological outcomes. ${ }^{25}$ Besides, several decision aids on management options of breast cancer risk in BRCA1/2 mutation carriers have been developed and tested. ${ }^{26-29}$ Women who used these decision aids also reported less decisional conflict, reduced uncertainty and cancer-specific distress, increased knowledge, and more satisfaction with their decision. ${ }^{26-29}$

In conclusion, our systematically developed patient decision aid for premenopausal BRCA1/2 mutation carriers participating in a Dutch clinical preference trial (NCT02321228) appears to be acceptable and usable according to both patients and professionals. However, whether its use really lowers decisional conflict, increases patient satisfaction, and results in informed decisions that are congruent with personal values has to be studied yet. Moreover, the rapidly growing body of evidence for this particular population will require regular updates of the content of the patient decision aid. 


\section{Acknowledgments}

The authors would like to thank all patients, patient advocates and clinicians who reviewed several drafts of the decision aid. The development of this patient decision aid was supported by a research grant from the Dutch Cancer Society as part of grant number KUN 2014-7187. 


\section{REFEREMCES}

1. Chen S, Parmigiani G. Meta-analysis of BRCA1 and BRCA2 penetrance. J Clin Oncol 2007; 25(11): 1329-33.

2. Rebbeck TR, Kauff ND, Domchek SM. Meta-analysis of risk reduction estimates associated with riskreducing salpingo-oophorectomy in BRCA1 or BRCA2 mutation carriers. J Natl Cancer Inst 2009; 101(2): 80-7.

3. Marchetti C, De Felice F, Palaia I, et al. Risk-reducing salpingo-oophorectomy: a meta-analysis on impact on ovarian cancer risk and all cause mortality in BRCA 1 and BRCA 2 mutation carriers. BMC Womens Health 2014; 14(1): 150.

4. Russo A, Calo V, Bruno L, Rizzo S, Bazan V, Di Fede G. Hereditary ovarian cancer. Crit Rev Oncol Hematol 2009; 69(1): 28-44.

5. Arts-de Jong M, Maas AH, Massuger LF, Hoogerbrugge N, de Hullu JA. BRCA1/2 mutation carriers are potentially at higher cardiovascular risk. Crit Rev Oncol Hematol 2014.

6. Parker WH, Feskanich D, Broder MS, et al. Long-term mortality associated with oophorectomy compared with ovarian conservation in the nurses' health study. Obstet Gynecol 2013; 121(4): 709-16.

7. Greene MH, Mai PL, Schwartz PE. Does bilateral salpingectomy with ovarian retention warrant consideration as a temporary bridge to risk-reducing bilateral oophorectomy in BRCA1/2 mutation carriers? Am J Obstet Gynecol 2011; 204(1): 19 e1-6.

8. $\quad$ Arts-de Jong M, Harmsen MG, Hoogerbrugge N, Massuger LF, Hermens RP, de Hullu JA. Risk-reducing salpingectomy with delayed oophorectomy in BRCA1/2 mutation carriers: patients' and professionals' perspectives. Gynecol Oncol 2015; 136 (2): 305-10.

9. Holman LL, Friedman S, Daniels MS, Sun CC, Lu KH. Acceptability of prophylactic salpingectomy with delayed oophorectomy as risk-reducing surgery among BRCA mutation carriers. Gynecol Oncol 2014; 133(2): 283-6.

10. Piek JM, van Diest PJ, Zweemer RP, et al. Dysplastic changes in prophylactically removed Fallopian tubes of women predisposed to developing ovarian cancer. J Pathol 2001; 195(4): 451-6.

11. Mingels MJ, Roelofsen T, van der Laak JA, et al. Tubal epithelial lesions in salpingo-oophorectomy specimens of BRCA-mutation carriers and controls. Gynecol Oncol 2012; 127(1): 88-93.

12. Kurman RJ. Origin and molecular pathogenesis of ovarian high-grade serous carcinoma. Ann Oncol 2013; 24 Suppl 10: x16-21.

13. Harmsen MG, Arts-de Jong M, Hoogerbrugge N, et al. Early salpingectomy (TUbectomy) with delayed oophorectomy to improve quality of life as alternative for risk-reducing salpingo-oophorectomy in BRCA1/2 mutation carriers (TUBA study): a prospective non-randomised multicentre study. BMC Cancer 2015; 15: 593.

14. Stacey $\mathrm{D}$, Legare $\mathrm{F}$, Col NF, et al. Decision aids for people facing health treatment or screening decisions. Cochrane Database Syst Rev 2014; (1): CD001431.

15. Coulter A, Stilwell D, Kryworuchko J, Mullen PD, Ng CJ, van der Weijden T. A systematic development process for patient decision aids. BMC Med Inform Decis Mak 2013; 13 Suppl 2: S2.

16. Elwyn G, O'Connor A, Stacey D, et al. Developing a quality criteria framework for patient decision aids: online international Delphi consensus process. BMJ 2006; 333(7565): 417.

17. Harmsen MG, IntHout J, Arts-de Jong M, et al. Salpingectomy With Delayed Oophorectomy in BRCA1/2 Mutation Carriers: Estimating Ovarian Cancer Risk. Obstet Gynecol 2016; 127(6): 1054-63.

18. Schoorel EN, Vankan E, Scheepers HC, et al. Involving women in personalised decision-making on mode of delivery after caesarean section: the development and pilot testing of a patient decision aid. BJOG 2014; 121 (2): 202-9.

19. Chandrasekaran D, Menon U, Evans G, et al. Risk reducing salpingectomy and delayed oophorectomy in high risk women: views of cancer geneticists, genetic counsellors and gynaecological oncologists in the UK. Fam Cancer 2015. 
20. Finch A, Evans G, Narod SA. BRCA carriers, prophylactic salpingo-oophorectomy and menopause: clinical management considerations and recommendations. Women's health 2012; 8(5): 543-55.

21. Marchetti C, ladarola R, Palaia I, et al. Hormone therapy in oophorectomized BRCA1/2 mutation carriers. Menopause 2014; 21(7): 763-8.

22. Witteman HO, Gavaruzzi T, Scherer LD, et al. Effects of Design Features of Explicit Values Clarification Methods: A Systematic Review. Med Decis Making 2016; 36(6): 760-76.

23. van Peperstraten AM, Hermens RP, Nelen WL, et al. Deciding how many embryos to transfer after in vitro fertilisation: development and pilot test of a decision aid. Patient Educ Couns 2010; 78(1): 124-9.

24. van Roosmalen MS, Stalmeier PF, Verhoef $L C$, et al. Randomised trial of a decision aid and its timing for women being tested for a BRCA1/2 mutation. Br J Cancer 2004; 90 (2): 333-42.

25. Tiller K, Meiser B, Gaff C, et al. A randomized controlled trial of a decision aid for women at increased risk of ovarian cancer. Med Decis Making 2006; 26(4): 360-72.

26. Metcalfe KA, Poll A, O'Connor A, et al. Development and testing of a decision aid for breast cancer prevention for women with a BRCA1 or BRCA2 mutation. Clin Genet 2007; 72(3): 208-17.

27. Schwartz MD, Valdimarsdottir HB, DeMarco TA, et al. Randomized trial of a decision aid for BRCA1/BRCA2 mutation carriers: impact on measures of decision making and satisfaction. Health Psychol 2009; 28(1): $11-9$.

28. Hooker GW, Leventhal KG, DeMarco T, et al. Longitudinal changes in patient distress following interactive decision aid use among BRCA1/2 carriers: a randomized trial. Med Decis Making 2011; 31(3): 412-21.

29. Metcalfe KA, Dennis CL, Poll A, et al. Effect of decision aid for breast cancer prevention on decisional conflict in women with a BRCA1 or BRCA2 mutation: a multisite, randomized, controlled trial. Genet Med 2016. 


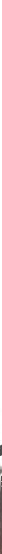

General discussion and future perspectives

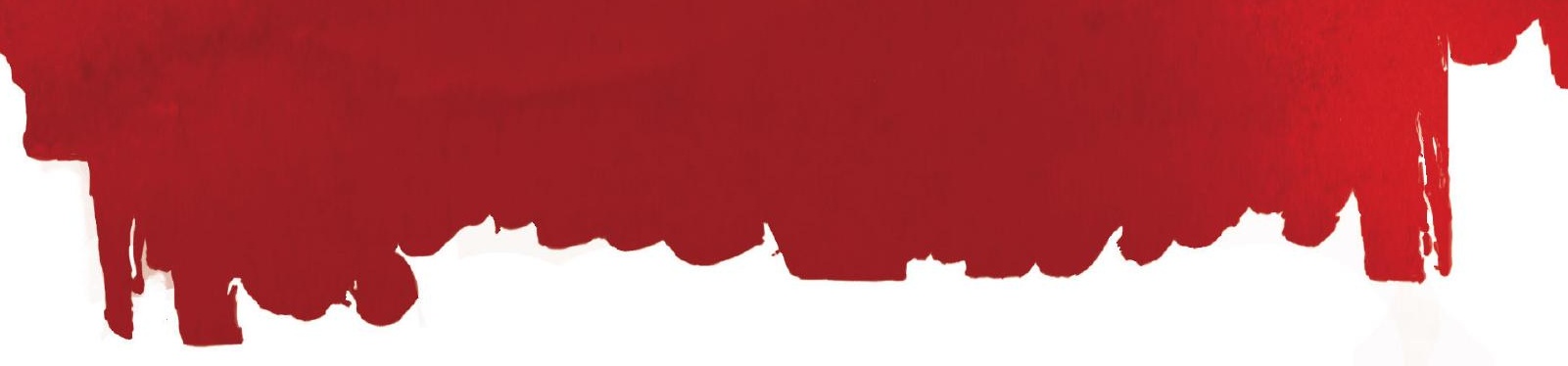


The focus of this thesis is on risk management of ovarian carcinoma in germline BRCA1/2 mutation carriers. Women who are aware of their elevated cancer risk because of such a mutation are considered "cancer previvors". Besides the purpose of preventing them from developing ovarian carcinoma by several risk-reducing strategies, increasing importance is attached to previvors' quality of life. This is in parallel with cancer survivors for whom quality of life has increasingly been brought into focus since survival rates have improved for many types of cancer, e.g. breast cancer. In this chapter, the main findings of this thesis are presented and discussed in the light of knowledge acquired composing this thesis and existing literature. Furthermore, the methods that were used are discussed and future perspectives are provided.

\section{MAIV RESULTS}

- Quality of life of BRCA1/2 mutation carriers is mostly impaired by premature menopause resulting from risk-reducing salpingo-oophorectomy (RRSO). Hormone replacement therapy does not fully alleviate climacteric symptoms, especially sexual symptoms. Furthermore, not all BRCA1/2 mutation carriers are eligible for hormone replacement therapy mainly due to a personal breast cancer history.

- Deciding not to undergo RRSO is highly exceptional in BRCA1/2 mutation carriers counseled at the Family Cancer Clinic of our institution and almost all of them have RRSO at the currently recommended age, if applicable.

- Metachronous peritoneal carcinomatosis after risk-reducing surgery in BRCA1/2 mutation carriers occurred mostly in BRCA1 mutation carriers, within 5 years after risk-reducing surgery, and when risk-reducing surgery was performed at older age. A role for the fallopian tube in the pathogenesis of primary peritoneal carcinoma was supported by the interval between risk-reducing surgery and diagnosis of peritoneal carcinomatosis, and the frequent detection of STICS.

- The differences in estimated ovarian carcinoma risks between standard RRSO and the proposed alternative strategy consisting of salpingectomy with delayed oophorectomy are small when oophorectomy is delayed by 5 years, even when salpingectomy would be completely ineffective in reducing the risk of ovarian carcinoma.

- The uncertainty about the effect of risk-reducing salpingectomy with delayed oophorectomy and the ease of the decision to undergo RRSO were indicated as important barriers for the alternative strategy among BRCA1/2 mutation carriers and professionals. The main facilitator mentioned by both was postponement of surgical menopause.

- The majority of BRCA1/2 mutation carriers and their professionals were willing to participate in a clinical trial on risk-reducing salpingectomy with delayed oophorectomy under the consideration that it would not be a randomized study. Therefore, we designed 
the TUBA study: a currently ongoing non-randomized clinical preference trial comparing salpingectomy with delayed oophorectomy with standard RRSO in terms of menopauserelated quality of life and several secondary outcome measures. As part of this TUBA study, we developed a patient decision aid to support BRCA1/2 mutation carriers who face the choice between salpingectomy with delayed oophorectomy and RRSO in this trial. The final version met 37 of 43 IPDAS criteria for content and development process and was found clear, balanced and comprehensible in pilot tests among BRCA1/2 mutation carriers and professionals.

\section{DISCUSSIOH OF THE MAIM RESULTS}

As shown in chapter 2, premenopausal RRSO is an important factor that can affect quality of life of BRCA1/2 mutation carriers.' Nearly all previvors at our institution undergo RRSO before the age of 45 , as evaluated in chapter 3 , and may thus face several quality of life issues. ${ }^{2}$ The alternative strategy to reduce the risk of ovarian carcinoma among BRCA1/2 mutation carriers proposed in this thesis, consisting of salpingectomy with delayed oophorectomy, aims to improve menopauserelated quality of life in ovarian carcinoma previvors by delaying premature menopause. ${ }^{3}$ However, the effect on ovarian carcinoma risk is still unclear and can only be estimated (chapter 6 ). ${ }^{4}$ In other words, the challenge is to weigh pros and cons of RRSO in terms of implications for quality of life and carcinoma risk. Accurate estimation of this risk is crucial in this consideration.

\section{Estimated ovarian and breast carcinoma risks in BRCA1/2 mutation carriers}

Estimated carcinoma risks for two different risk-reducing strategies play a central role in this thesis: on the one hand, for standard RRSO and on the other hand, for salpingectomy with delayed oophorectomy. Despite our utmost effort to estimate risks as accurately as possible, several considerations have to be made when interpreting these risk estimates.

\section{Cumulative risk of ovarian carcinoma}

In this thesis, cumulative carcinoma risks published by Chen and Parmigiani in a meta-analysis formed the basis for most hypotheses we elaborated on. ${ }^{5}$ Although this is a frequently cited metaanalysis, large heterogeneity existed between included studies that could not be well explained by its authors. Studies were performed in different populations with their own prevalence of specific (founder) mutations. ${ }^{6}$ Furthermore, included studies that were published earlier reported higher penetrance of BRCA1/2 genes than more recent studies which might be explained by the criteria for genetic testing. ${ }^{6}$ Genetic testing was primarily offered to women with a strong family history, which is known to increase carcinoma risk.7.8 Furthermore, the use of different risk estimation methods and bias corrections across studies may be responsible for the diversity of reported cumulative risks of ovarian carcinoma, as has been shown for breast cancer. ${ }^{9}$ 
In addition, we assumed that the same mean cumulative risk for BRCA1/2 mutation carriers applies to every single BRCA1/2 mutation carrier throughout this thesis. However, a lot of risk modifiers have already been identified telling us that heterogeneity exists even within a group of women carrying a germline mutation in the same gene. First, the risk of ovarian carcinoma is influenced by the type and position of the mutation: mutations in the ovarian carcinoma cluster region are associated with an increased risk.,10,11 As far as we know, however, this has not changed risk counseling until now. Second, the risk can be further modified by other risk-modifying genes or by certain single nucleotide polymorphisms (SNPs). ${ }^{12-15}$ Third, several reproductive factors have been associated with reduced ovarian carcinoma risk in mutation carriers: tubal ligation, breastfeeding and increasing parity in BRCA1 mutation carriers, and use of oral contraceptives in both BRCA1 and BRCA2 mutation carriers. ${ }^{16}$ Taking all these factors into account may result in individualized risk estimates contributing to more tailored counseling in cancer risk management: a relatively low or high personal residual risk may be a reason for women to choose for one risk-reducing strategy or the other.

\section{Residual risk of ovarian carcinoma after risk-reducing salpingo-oophorectomy}

Besides the wide divergence in reported cumulative risk of ovarian carcinoma in BRCA1/2 mutation carriers, a variety also exists in reported effect sizes of RRSO and residual risk of primary peritoneal carcinoma occurring after RRSO. Understandably, this is another important issue in decisionmaking regarding interventions to lower the risk of ovarian carcinoma.

There are several ways to present the residual risk of primary peritoneal carcinoma. Some authors reported the percentage of BRCA1/2 mutation carriers who were diagnosed with primary peritoneal carcinoma after RRSO in their cohort studies: $0.3-1.1 \% .{ }^{17-23}$ Those cohorts varied in terms of mean or median age at RRSO (42 to 49 years) and duration of follow-up (22-109 months). Finch et al. ${ }^{21}$ differentiated for age at RRSO: 0.3\% developed primary peritoneal carcinoma after RRSO undergone before the age of 40 years and $0.7 \%$ after RRSO undergone between 40 and 50 years old during a mean follow-up of approximately 6 years. Based on these findings, they calculated the cumulative risk of primary peritoneal carcinoma in the first 20 years after RRSO: 3.9\% for BRCA1 mutation carriers and 1.9\% for BRCA2 mutation carriers. ${ }^{21}$ Earlier, Casey et al. ${ }^{24}$ estimated this risk also at 3.9\% for BRCA1 mutation carriers. Another way to report the effect of risk-reducing surgery is by hazard ratios. Several of the aforementioned studies report hazard ratios for ovarian carcinoma after RRSO between 0.04 and 0.28, corresponding to $96 \%$ and $72 \%$ risk reduction, respectively. Two meta-analyses by Rebbeck et al..$^{25}$ and Marchetti et al. ${ }^{26}$ calculated hazard ratios of 0.21 (95\% Confidence Interval (Cl) 0.12 to 0.39 ) and 0.19 (95\% Cl 0.13 to 0.27). ${ }^{17,19,21,25-27}$ Although it seems reasonable that RRSO at younger age results in lower hazard ratios, sufficient data lack to calculate separate hazard ratios for certain ages at RRSO. Therefore, we assumed the hazard ratios to be stable regardless of age at RRSO throughout this thesis, i.e. hazard ratios of 0.20 and 0.04 corresponding to $80 \%$ and $96 \%$ risk reduction after RRSO, respectively. Follow-up of women undergoing RRSO 
at the recommended age may reveal a more accurate estimate of the residual risk for a more homogeneous group in terms of mutation (BRCA1 or BRCA2) and age.

\section{Residual risk of ovarian carcinoma after risk-reducing salpingectomy}

As repeatedly mentioned in this thesis, no clinical data on the effect of salpingectomy on the risk of ovarian carcinoma are available to date. We assumed a potential maximum effect based on available indirect evidence (chapter 6). ${ }^{4}$ The effectiveness in terms of menopause-related quality of life of delaying oophorectomy after salpingectomy is the primary outcome of the ongoing TUBA study (chapter 7). ${ }^{3}$ However, we will not be able to draw conclusions whether salpingectomy with delayed oophorectomy is non-inferior (or even superior) to standard RRSO in terms of the residual risk of ovarian carcinoma, mainly because of two reasons. At first, Anderson et al. ${ }^{28}$ calculated the sample size that would be needed in a randomized controlled trial of equivalence between RRSO and risk-reducing salpingectomy. Assuming 95\% risk reduction for RRSO, 3980 BRCA1 mutation carriers are needed in each arm. Including BRCA2 mutation carriers would require even more patients per arm, given the lower incidence of ovarian carcinoma in that group. Enrolling these numbers of study participants in a reasonable time interval is not feasible in the Dutch population. At second, although a randomized controlled trial would be the right study design, BRCA1/2 mutation carriers nor healthcare professionals find randomization acceptable in such a clinical trial (chapter 5). ${ }^{29}$ Therefore, the TUBA study is the first step towards clinical data on the efficacy and safety of salpingectomy with delayed oophorectomy in BRCA1/2 mutation carriers and it will prevent healthcare providers to perform salpingectomies outside a clinical trial (without close and structural follow-up). Nevertheless, more initiatives and international collaborations on this topic will be necessary to be able to study the real effect of salpingectomy on ovarian carcinoma incidence in a sufficient number of BRCA1/2 mutation carriers.

\section{Effect of RRSO on breast cancer risk}

Until recently, it was widely accepted that RRSO before menopause reduced BRCA1/2 mutation carriers' risk of developing breast cancer approximately by half.19,27,30,31 However, the four studies in which this risk-reducing effect was found used various study designs and eligibility criteria and some of the study characteristics could have caused bias in several ways, which was addressed by Heemskerk-Gerritsen et al. in 2015.32 They applied all four methods of these studies to their own Dutch cohort of BRCA1/2 mutation carriers and found effect sizes similar to the original studies. Thereupon, they proposed an alternative analytical method thereby minimizing the potential sources of selection bias. Applying this alternative method to the same Dutch cohort resulted in no statistically significant risk reduction of breast cancer after RRSO. ${ }^{32}$ Judging by the strength of this evidence, we assumed no effect of RRSO on breast cancer risk in this thesis. Nevertheless, the authors commented that the total number of BRCA2 mutation carriers and the number of events in this study were too small to draw conclusions for the group of BRCA2 mutation carriers alone. 
Kotsopoulos et al. ${ }^{33}$ did find a statistically significant risk reduction in breast cancer diagnosed prior to age 50 years among BRCA2 mutation carriers who underwent RRSO (HR 0.18, 95\% Cl 0.05 to 0.63). However, there were only three cases of breast cancer in the RRSO group. ${ }^{33}$

BRCA2 mutation carriers are known to have more estrogen receptor (ER) positive breast cancer compared to BRCA1 mutation carriers. ${ }^{34}$ Therefore, a difference in the effect of RRSO on breast cancer incidence between BRCA1 and BRCA2 is quite conceivable. Further studies with a larger number of BRCA2 mutation carriers and extended duration of follow-up are needed to find out whether a risk-reducing effect of RRSO does exist in the subgroup of BRCA2 mutation carriers. If such an effect does exist, it should be taken into account when counseling women from this group on RRSO versus salpingectomy with delayed oophorectomy and it should be included in an updated version of the patient decision aid that we developed (chapter 8).

\section{Carcinoma risks other than breast and ovarian}

Risk of endometrial carcinoma in BRCA1/2 mutation carriers

Up to now, it is uncertain whether BRCA1/2 mutation carriers also have an increased risk of endometrial carcinoma, especially BRCA1 mutation carriers. ${ }^{22,35-40}$ Internationally, RRSO is often accompanied by hysterectomy so that hormone replacement therapy with estrogens alone is sufficient and the potential increase of breast cancer risk by progestins is eliminated. However, evidence for a beneficial effect has been found insufficient to make hysterectomy part of standard risk-reducing surgery in the Netherlands until now: data on an elevated risk of endometrial carcinoma are not convincing and the intrauterine part of the fallopian tube has not been proven a preference site for the development of carcinoma of any type after RRSO.

In 2016, the publication by Shu et al. ${ }^{41}$ brought this discussion back to life. In a large prospective study, they found that BRCA1 mutation carriers had an increased risk of serous or serous-like endometrial carcinoma after RRSO without hysterectomy compared to the general population: observed versus expected ratio 22.2 (95\% Cl 6.1 to 56.9). They calculated that the cumulative risk of developing serous or serous-like endometrial carcinoma is $2.6 \%$ to $4.7 \%$ between RRSO at age 45 years and age 70 years. ${ }^{41}$ In comparison, the lifetime risk of developing any type of endometrial carcinoma is $2.8 \%$ in the general population. ${ }^{42}$ The role of tamoxifen exposure as potential risk modifier is still unclear but might (partially) explain the higher incidence in BRCA1 mutation carriers, although tamoxifen use has been associated with endometrioid rather than with serous endometrial carcinoma. Confirmation of these findings in other populations including the Dutch population is needed by the collection and analysis of detailed clinical and pathological data. Until then, there is not enough evidence to routinely incorporate hysterectomy into risk-reducing surgery for BRCA1/2 mutation carriers. 


\section{Alternatives to risk-reducing surgery}

In this thesis, we proposed an alternative surgical strategy to reduce the ovarian carcinoma risk of BRCA1/2 mutation carriers. However, any risk-reducing surgery would be less needed if premalignant or early invasive lesions could be detected. Screening methods with transvaginal ultrasound and measurement of serum CA125 have not resulted in improved survival rates in BRCA1/2 mutation carriers until now. ${ }^{43-45}$

In a recent report, however, a significant risk reduction of ovarian carcinoma mortality by annual measurement of serum CA125 was found in a large cohort of postmenopausal women from the United Kingdom general population after a median follow-up of 11 years. ${ }^{46}$ The pattern of CA125 levels over time was interpreted according to a risk of ovarian cancer algorithm rather than a fixed cut-off value. Mortality due to ovarian carcinoma was significantly reduced by $20 \%$ ( $95 \%$ Cl -2 to 40) when prevalent cases at the start of the screening program were excluded. However, mortality rates were very low: about $0.29 \%$ in the CA125 screened group and 0.34\% in the unscreened group. The authors note that further follow-up is needed to draw firm conclusions on efficacy and cost-effectiveness of this screening program. ${ }^{46}$ Furthermore, results from the general population cannot simply be extrapolated to the high-risk population of BRCA1/2 mutation carriers.

To detect (pre)malignant lesions, a test easily performed at the outpatient department or even by the patient herself would be preferred. One could think of the use of Papanicolaou (Pap) tests or tampons to collect cells of vaginal, cervical, endometrial, and hopefully even tubal or ovarian origin. These samples may subsequently be analyzed for TP53 mutations: a detected mutation can be the clue for precursor lesions or early invasive carcinoma of the upper genital tract. This principle has already been described for patients with ovarian carcinoma not necessarily carrying a BRCA mutation. ${ }^{47,48}$ In 9 of 22 (41\%) ovarian carcinomas (FIGO stage IA to IV), identical mutations were detected in both the tumor itself and the Pap smear. ${ }^{47}$ In another study by the same group, 3 of 5 (60\%) stage IIIC ovarian carcinoma patients without previous tubal ligation identical TP53 mutations were detected in tumor DNA and DNA extracted from a tampon that had been placed in the vagina 8-12 hours before surgery. ${ }^{48}$ On these small numbers, conclusion cannot be drawn. Sensitivity is not convincing and might be far worse for premalignant lesions compared to fullblown ovarian carcinomas. In an ongoing study from our center (NTR4299), samples from patients with endometrial or ovarian carcinoma are collected by Pap smear, endometrial biopsy (Pipelle) and a brush-based cervicovaginal self-sample. DNA from these samples will be extracted and compared to tissue DNA from the primary tumor. Still, more studies and further development of advanced techniques are needed.

Another test to detect serous tubal intraepithelial carcinomas (STICs) in women at high risk of highgrade serous ovarian carcinoma is currently under investigation in an international multicenter trial: the LUSTIC study (NCT02039388). Aim of the study is to detect TP53 mutations from STICs in fluid retrieved after lavage of the uterine cavity. Lavage is performed right before RRSO with or without hysterectomy. Yet, no (preliminary) results are available. 


\section{FUTURE PERSPECTIVES}

\section{Implications for future research}

Minimizing the number of BRCA1/2 mutation carriers with impaired quality of life due to the consequences of RRSO is an important goal for future research in this field, next to effective methods to reduce carcinoma risk.

One way of diminishing the number of women who face premature menopause is finding reasonable alternatives to risk-reducing surgery. To obtain better survival rates, further attempts are needed to improve early detection of ovarian carcinoma. Several options have been already discussed in the paragraph on alternatives to risk-reducing surgery. Additionally, improvement and individualization of hormone replacement therapy may also alleviate adverse effects of RRSO. Ideally, randomized controlled trials should be carried out to investigate safety of hormone replacement therapy and to evaluate the optimal type and duration of hormone replacement therapy for subgroups within the BRCA1/2 population. Non-medical interventions to cope with psychosocial and sexual consequences of RRSO are also promising and currently under investigation in the Dutch PURSUE study: Psychosexual Consequences of RRSO in BRCA1/2 Mutation Carriers (NCT02372864). The effect of salpingectomy and its future role in reducing the risk of ovarian carcinoma among BRCA1/2 mutation carriers needs further investigation. Our ongoing TUBA study will answer the question on its efficacy in improving quality of life. Besides, it will give us clues on safety regarding ovarian carcinoma risk. Results from some other ongoing studies will contribute to our knowledge on salpingectomy as risk-reducing option. In the United States, compliance with salpingectomy and oophorectomy delayed for three years is being investigated (NCT01907789). Eligible are premenopausal BRCA1/2 mutation carriers aged 47 years or younger. The same study group is evaluating sexual functioning and quality of life after salpingectomy with delayed oophorectomy and compares it to women undergoing standard RRSO (NCT02760849). Women are eligible between 30 and 50 years old and when they carry a germline mutation in an ovarian carcinoma gene including BRCA1/2. A French study group looks at the rate of pelvic carcinoma occurring between radical fimbriectomy and menopause in women at high risk for ovarian carcinoma who are reluctant to undergo oophorectomy. As previously mentioned, more initiatives and international collaborations on salpingectomy are necessary to determine its entire effect in a sufficient number of BRCA1/2 mutation carriers. An international consortium needs to be established to coordinate studies as mentioned in this paragraph and to match outcome measures and methods of these studies. Only then we might be able to find out the full effect and safety of salpingectomy with delayed oophorectomy in BRCA1/2 mutation carriers.

Additionally, more data on risk modifiers of ovarian carcinoma risk in BRCA1/2 mutation carriers are needed to facilitate risk tailoring. We expect that individualized estimations of the (residual) cumulative lifetime risk of ovarian carcinoma or either the cumulative risk over the next 10 years, for example, will be crucial in decision-making on ovarian carcinoma risk management. 
Furthermore, the exact effects of premature menopause have to be further elucidated. Cardiovascular effects after RRSO, for example, are currently under investigation in a study initiated by our research group: CArdiovascular Risk after risk-reducing Salpingo-Oophorectomy in BrCA mutation carriers (CARSOBRA study, NL51609.091.15). Traditional cardiovascular risk factors are investigated and non-invasive measurements of atherosclerosis (carotid intima media thickness and pulse wave velocity) are performed in BRCA1/2 mutation carriers who underwent RRSO at age 45 or younger and at least 5 years ago.

It is still under debate whether BRCA1/2 mutation carriers have an earlier age of onset of natural menopause. ${ }^{49}$ This might be more relevant for reproductive issues (e.g. ovarian reserve) than for risk-reducing issues since RRSO is currently recommended several years before the average age of natural menopause onset. However, it might become important again when oophorectomy after previous salpingectomy can be delayed until natural menopause commences or ovaries can be indefinitely retained.

Writing about reproductive issues, women might also benefit when delaying risk-reducing surgery, especially oophorectomy, would be proven safe. In our center, we experience an increasing demand for fertility preservation options among BRCA1/2 mutation carriers who reached the currently recommended age for RRSO but still have the desire to become pregnant. The place of several methods of fertility preservation (e.g. cryopreservation of oocytes or ovarian tissue) for this indication and issues like the upper age of cryopreservation should be further explored.

Finally, if we consider one step ahead of reducing cancer risks in BRCA1/2 mutation carriers, we can think of preventing offspring from inheriting the mutated gene. Two approaches are available now: prenatal diagnosis with the possibility to terminate pregnancy when the mutation is found in fetal DNA, and pre-implantation genetic diagnosis (PGD) testing embryos obtained by in vitro fertilization (IVF/ICSI) for the presence of the BRCA mutation and only transferring the unaffected embryos into the uterus..$^{50}$ However, both approaches involve several ethical issues and safety regarding breast cancer risk due to hormonal stimulation has to be further confirmed for PGD. ${ }^{50,51}$ Larger studies and studies on new techniques with are future challenges.

\section{Implications for clinical practice}

As long as the efficacy and safety of the alternative strategy of salpingectomy with delayed oophorectomy in BRCA1/2 mutation carriers has not been proven, it should exclusively be performed within the context of a clinical trial (e.g. the TUBA study) with close and structured follow-up. Our patient decision aid may be offered to BRCA1/2 mutation carriers who face the choice between standard RRSO and salpingectomy with delayed oophorectomy to fully inform them on the two strategies and in attempt to facilitate their decision-making process. 


\section{REFEREMCES}

1. Harmsen MG, Hermens RP, Prins JB, Hoogerbrugge N, de Hullu JA. How medical choices influence quality of life of women carrying a BRCA mutation. Crit Rev Oncol Hematol 2015; 96(3): 555-68.

2. Harmsen $M G$, Arts-de Jong M, Horstik K, et al. Very high uptake of risk-reducing salpingo-oophorectomy in BRCA1/2 mutation carriers: A single-center experience. Gynecol Oncol 2016; 143(1): 113-9.

3. Harmsen MG, Arts-de Jong M, Hoogerbrugge N, et al. Early salpingectomy (TUbectomy) with delayed oophorectomy to improve quality of life as alternative for risk-reducing salpingo-oophorectomy in BRCA1/2 mutation carriers (TUBA study): a prospective non-randomised multicentre study. BMC Cancer 2015; 15: 593.

4. Harmsen MG, IntHout J, Arts-de Jong M, et al. Salpingectomy With Delayed Oophorectomy in BRCA1/2 Mutation Carriers: Estimating Ovarian Cancer Risk. Obstet Gynecol 2016; 127(6): 1054-63.

5. Chen S, Parmigiani G. Meta-analysis of BRCA1 and BRCA2 penetrance. J Clin Oncol 2007; 25(11): 1329-33.

6. De Bock GH, Mourits MJ, Oosterwijk JC. One risk fits all? J Clin Oncol 2007; 25(22): 3383-4; author reply 4.

7. Teixeira N, Mourits MJ, Vos JR, et al. Ovarian cancer in BRCA1/2 mutation carriers: The impact of mutation position and family history on the cancer risk. Maturitas 2015; 82 (2): 197-202.

8. Metcalfe K, Lubinski J, Lynch HT, et al. Family history of cancer and cancer risks in women with BRCA1 or BRCA2 mutations. J Natl Cancer Inst 2010; 102 (24): 1874-8.

9. Vos JR, Hsu L, Brohet RM, et al. Bias Correction Methods Explain Much of the Variation Seen in Breast Cancer Risks of BRCA1/2 Mutation Carriers. J Clin Oncol 2015; 33(23): 2553-62.

10. Finkelman BS, Rubinstein WS, Friedman $\mathrm{S}$, et al. Breast and ovarian cancer risk and risk reduction in Jewish BRCA1/2 mutation carriers. J Clin Oncol 2012; 30(12): 1321-8.

11. Rebbeck TR, Mitra N, Wan F, et al. Association of type and location of BRCA1 and BRCA2 mutations with risk of breast and ovarian cancer. JAMA : the journal of the American Medical Association 2015; 313(13): 1347-61.

12. Kontorovich T, Levy A, Korostishevsky M, Nir U, Friedman E. Single nucleotide polymorphisms in miRNA binding sites and miRNA genes as breast/ovarian cancer risk modifiers in Jewish high-risk women. Int J Cancer 2010; 127(3): 589-97.

13. Bojesen SE, Pooley KA, Johnatty SE, et al. Multiple independent variants at the TERT locus are associated with telomere length and risks of breast and ovarian cancer. Nat Genet 2013; 45(4): 371-84, 84e1-2.

14. Vigorito E, Kuchenbaecker KB, Beesley J, et al. Fine-Scale Mapping at 9p22.2 Identifies Candidate Causal Variants That Modify Ovarian Cancer Risk in BRCA1 and BRCA2 Mutation Carriers. PLoS One 2016; 11(7): e0158801.

15. Benitez-Buelga C, Vaclova T, Ferreira S, et al. Molecular insights into the OGG1 gene, a cancer risk modifier in BRCA1 and BRCA2 mutations carriers. Oncotarget 2016; 7(18): 25815-25.

16. Friebel TM, Domchek SM, Rebbeck TR. Modifiers of cancer risk in BRCA1 and BRCA2 mutation carriers: systematic review and meta-analysis. J Natl Cancer Inst 2014; 106(6): dju091.

17. Rebbeck TR, Lynch HT, Neuhausen SL, et al. Prophylactic oophorectomy in carriers of BRCA1 or BRCA2 mutations. N Engl J Med 2002; 346(21): 1616-22.

18. Meeuwissen PA, Seynaeve C, Brekelmans CT, Meijers-Heijboer HJ, Klijn JG, Burger CW. Outcome of surveillance and prophylactic salpingo-oophorectomy in asymptomatic women at high risk for ovarian cancer. Gynecol Oncol 2005; $97(2):$ 476-82.

19. Domchek SM, Friebel TM, Singer CF, et al. Association of risk-reducing surgery in BRCA1 or BRCA2 mutation carriers with cancer risk and mortality. JAMA : the journal of the American Medical Association 2010; 304(9): 967-75.

20. Powell CB, Chen LM, McLennan J, et al. Risk-reducing salpingo-oophorectomy (RRSO) in BRCA mutation carriers: experience with a consecutive series of 111 patients using a standardized surgical-pathological protocol. Int J Gynecol Cancer 2011; 21(5): 846-51. 
21. Finch AP, Lubinski J, Moller P, et al. Impact of oophorectomy on cancer incidence and mortality in women with a BRCA1 or BRCA2 mutation. J Clin Oncol 2014; 32(15): 1547-53.

22. Reitsma W, Mourits MJ, de Bock GH, Hollema $\mathrm{H}$. Endometrium is not the primary site of origin of pelvic high-grade serous carcinoma in BRCA1 or BRCA2 mutation carriers. Mod Pathol 2013; 26(4): 572-8.

23. Bacha OM, Gregoire J, Grondin K, et al. Effectiveness of risk-reducing salpingo-oophorectomy in preventing ovarian cancer in a high-risk French Canadian population. Int J Gynecol Cancer 2012; 22(6): 974-8.

24. Casey MJ, Synder C, Bewtra C, Narod SA, Watson P, Lynch HT. Intra-abdominal carcinomatosis after prophylactic oophorectomy in women of hereditary breast ovarian cancer syndrome kindreds associated with BRCA1 and BRCA2 mutations. Gynecol Oncol 2005; 97 (2): 457-67.

25. Rebbeck TR, Kauff ND, Domchek SM. Meta-analysis of risk reduction estimates associated with riskreducing salpingo-oophorectomy in BRCA1 or BRCA2 mutation carriers. J Natl Cancer Inst 2009; 101(2): 80-7.

26. Marchetti C, De Felice F, Palaia I, et al. Risk-reducing salpingo-oophorectomy: a meta-analysis on impact on ovarian cancer risk and all cause mortality in BRCA 1 and BRCA 2 mutation carriers. BMC Womens Health 2014; 14(1): 150.

27. Kauff ND, Domchek SM, Friebel TM, et al. Risk-reducing salpingo-oophorectomy for the prevention of BRCA1- and BRCA2-associated breast and gynecologic cancer: a multicenter, prospective study. J Clin Oncol 2008; 26(8): 1331-7.

28. Anderson CK, Wallace S, Guiahi M, Sheeder J, Behbakht K, Spillman MA. Risk-reducing salpingectomy as preventative strategy for pelvic serous cancer. Int J Gynecol Cancer 2013; 23(3): 417-21.

29. Arts-de Jong M, Harmsen MG, Hoogerbrugge N, Massuger LF, Hermens RP, de Hullu JA. Risk-reducing salpingectomy with delayed oophorectomy in BRCA1/2 mutation carriers: patients' and professionals' perspectives. Gynecol Oncol 2015; 136 (2): 305-10.

30. Eisen A, Lubinski J, Klijn J, et al. Breast cancer risk following bilateral oophorectomy in BRCA1 and BRCA2 mutation carriers: an international case-control study. J Clin Oncol 2005; 23(30): 7491-6.

31. Domchek SM, Friebel TM, Neuhausen SL, et al. Mortality after bilateral salpingo-oophorectomy in BRCA1 and BRCA2 mutation carriers: a prospective cohort study. Lancet Oncol 2006; 7(3): 223-9.

32. Heemskerk-Gerritsen BA, Seynaeve C, van Asperen CJ, et al. Breast cancer risk after salpingooophorectomy in healthy BRCA1/2 mutation carriers: revisiting the evidence for risk reduction. J Nat/ Cancer Inst 2015; 107(5).

33. Kotsopoulos J, Huzarski T, Gronwald J, et al. Bilateral Oophorectomy and Breast Cancer Risk in BRCA1 and BRCA2 Mutation Carriers. J Natl Cancer Inst 2017; 109(1).

34. Atchley DP, Albarracin CT, Lopez A, et al. Clinical and pathologic characteristics of patients with BRCApositive and BRCA-negative breast cancer. J Clin Oncol 2008; 26(26): 4282-8.

35. Thompson D, Easton DF, Breast Cancer Linkage C. Cancer Incidence in BRCA1 mutation carriers. J Natl Cancer Inst 2002; 94(18): 1358-65.

36. Breast Cancer Linkage C. Cancer risks in BRCA2 mutation carriers. J Nat/ Cancer Inst 1999; 91(15): 1310-6.

37. Segev Y, lqbal J, Lubinski J, et al. The incidence of endometrial cancer in women with BRCA1 and BRCA2 mutations: an international prospective cohort study. Gynecol Oncol 2013; 130(1): 127-31.

38. Bruchim I, Amichay K, Kidron D, et al. BRCA1/2 germline mutations in Jewish patients with uterine serous carcinoma. Int J Gynecol Cancer 2010; 20(7): 1148-53.

39. Lavie O, Ben-Arie A, Segev Y, et al. BRCA germline mutations in women with uterine serous carcinoma-still a debate. Int J Gynecol Cancer 2010; 20(9): 1531-4.

40. Pennington KP, Walsh T, Lee M, et al. BRCA1, TP53, and CHEK2 germline mutations in uterine serous carcinoma. Cancer 2013; 119(2): 332-8.

41. Shu CA, Pike MC, Jotwani AR, et al. Uterine Cancer After Risk-Reducing Salpingo-oophorectomy Without Hysterectomy in Women With BRCA Mutations. JAMA oncology 2016. 
42. National Cancer Institute. SEER Stat Fact Sheets: Endometrial Cancer. https://seer.cancer.gov/statfacts/ html/corp.html (accessed 11-21-2016.

43. Hermsen BB, Olivier RI, Verheijen RH, et al. No efficacy of annual gynaecological screening in BRCA1/2 mutation carriers; an observational follow-up study. Br J Cancer 2007; 96(9): 1335-42.

44. Woodward ER, Sleightholme HV, Considine AM, Williamson S, McHugo JM, Cruger DG. Annual surveillance by CA125 and transvaginal ultrasound for ovarian cancer in both high-risk and population risk women is ineffective. BJOG 2007; 114(12): 1500-9.

45. Oei AL, Massuger LF, Bulten J, Ligtenberg MJ, Hoogerbrugge N, de Hullu JA. Surveillance of women at high risk for hereditary ovarian cancer is inefficient. Br J Cancer 2006; 94 (6): 814-9.

46. Jacobs IJ, Menon U, Ryan A, et al. Ovarian cancer screening and mortality in the UK Collaborative Trial of Ovarian Cancer Screening (UKCTOCS): a randomised controlled trial. Lancet 2016; 387(10022): 945-56.

47. Kinde I, Bettegowda C, Wang Y, et al. Evaluation of DNA from the Papanicolaou test to detect ovarian and endometrial cancers. Sci Trans/ Med 2013; 5(167): 167ra4.

48. Erickson BK, Kinde I, Dobbin ZC, et al. Detection of somatic TP53 mutations in tampons of patients with high-grade serous ovarian cancer. Obstet Gynecol 2014; 124(5): 881-5.

49. van Tilborg TC, Broekmans FJ, Pijpe A, et al. Do BRCA1/2 mutation carriers have an earlier onset of natural menopause? Menopause 2016; 23(8): 903-10.

50. Derks-Smeets IA, de Die-Smulders CE, Mackens S, et al. Hereditary breast and ovarian cancer and reproduction: an observational study on the suitability of preimplantation genetic diagnosis for both asymptomatic carriers and breast cancer survivors. Breast Cancer Res Treat 2014; 145(3): 673-81.

51. Derks-Smeets IA, Gietel-Habets JJ, Tibben A, et al. Decision-making on preimplantation genetic diagnosis and prenatal diagnosis: a challenge for couples with hereditary breast and ovarian cancer. Hum Reprod 2014; 29(5): 1103-12. 



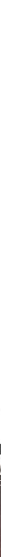

Summary / Samenvatting

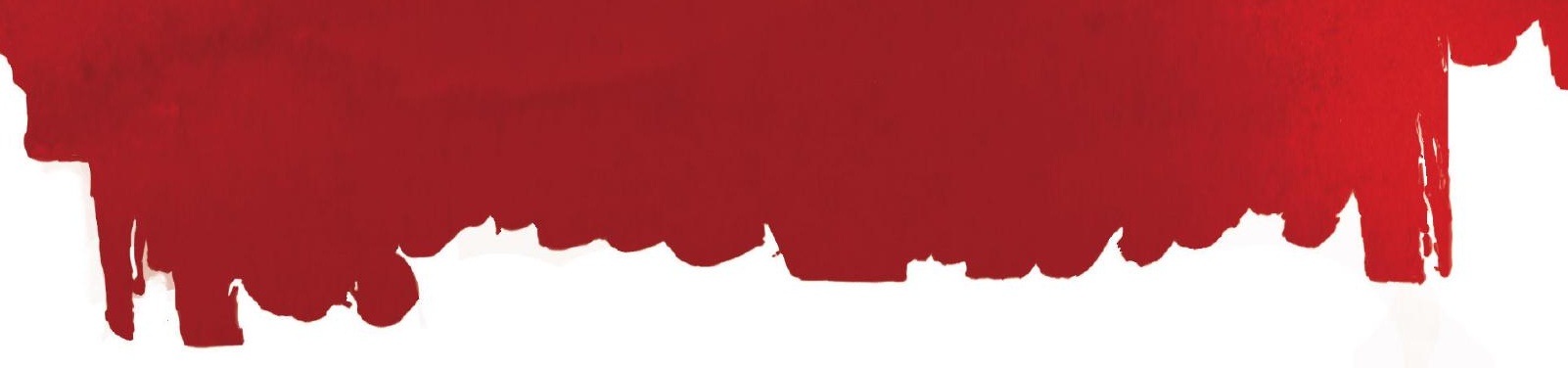




\section{SUMMARY}

This thesis discusses an alternative strategy to reduce the risk of ovarian carcinoma in BRCA1/2 mutation carriers. As we described in chapter 1, these women have an increased risk to develop ovarian carcinoma and the currently recommended intervention to reduce that risk is undergoing risk-reducing salpingo-oophorectomy (RRSO) around the age of 40 years. However, RRSO results in acute onset of surgical menopause with several adverse health consequences in short and long term. To improve quality of life and reduce side-effects of early surgical menopause, we explored the possibility of risk-reducing salpingectomy with delayed oophorectomy as alternative to RRSO allowing preservation of the ovaries and thereby postponement of premature menopause.

In chapter 2, we reviewed the literature to evaluate quality of life in unaffected BRCA mutation carriers and the influence of several choices they make with respect to cancer risk management. Overall, we found that general quality of life is not permanently affected in BRCA1/2 mutation carriers or by their choices for surveillance and/or risk-reducing surgery. However, premature menopause caused by RRSO affects (menopause-specific) quality of life most. Hormone replacement therapy does not fully alleviate climacteric symptoms and therefore, we concluded that there is a strong need for alternative strategies to reduce ovarian carcinoma risk and for improvements in postoperative care after RRSO, e.g., hormone replacement therapy and supportive care. We studied the uptake and timing of RRSO among BRCA1/2 mutation carriers in chapter $\mathbf{3}$ in order to evaluate how many women potentially face long-term health consequences after RRSO. We performed a single-center retrospective cohort study of BRCA1/2 mutation carriers diagnosed and counseled at the multidisciplinary Family Cancer Clinic of our institution between 1999 and 2014. Data of 580 BRCA1/2 mutation carriers were available for descriptive analyses and we found the uptake of RRSO to be $98.5 \%$ and $97.5 \%$ among BRCA1 and BRCA2 mutation carriers who are past the recommended age for RRSO, respectively. The vast majority undergoes RRSO $\leq 40$ years (90.8\% of BRCA1 mutation carriers) or $\leq 45$ years ( $97.3 \%$ of BRCA2 mutation carriers), provided that mutation status is known by that age. We concluded that the uptake of RRSO among BRCA1/2 mutation carriers who were counseled at our Family Cancer Clinic is extremely high, which might be largely attributed to the directive and uniform way of counseling by our professionals. The fact that RRSO in our population is often undergone at premenopausal age reinforced the need for minimization of long-term health consequences of premature surgical menopause, either by optimization of hormone replacement therapy or by inventing alternative strategies to RRSO. Despite RRSO, metachronous intra-abdominal carcinomatosis is occasionally diagnosed in $B R C A 1 / 2$ mutation carriers. We assembled cases to increase knowledge regarding occurrence and pathogenesis of intra-abdominal carcinomatosis after RRSO in chapter $\mathbf{4}$. The literature was searched for publications describing BRCA1/2 mutation carriers with peritoneal carcinomatosis after risk-reducing surgery and authors were requested to provide additional data. Cases were compared with an unselected single-institution control cohort of BRCA1/2 mutation carriers who 
underwent risk-reducing salpingo-oophorectomy between 2014 and 2016. We found that 31 of 36 collected cases (86.1\%) concerned BRCA1 mutation carriers. Median age was 52 years (range 30-71) at risk-reducing surgery and 60 years (range 37-75) at peritoneal carcinomatosis diagnosis, and median interval between those two was 54.5 months (range 11-292). The vast majority of peritoneal carcinomatosis was of the high-grade serous subtype. Histopathological details of RRSO specimens including the complete fallopian tubes were retrieved in 8 cases: 5 (62.5\%) with serous tubal intraepithelial carcinoma (STIC), one with epithelial atypia. Cases were statistically significantly older $(p=0.029)$ at risk-reducing surgery and harbored more STICs $(p<0.001)$ than women from the control cohort. We concluded that metachronous peritoneal carcinomatosis after risk-reducing surgery occurs predominantly in BRCA1 mutation carriers, within 5 years. Data suggest that surgery at younger age might lower rates of peritoneal carcinomatosis. These data can be used in gynecologic counseling of BRCA1/2 mutation carriers. RRSO should include complete salpingectomy and detailed histopathological examination of specimens removed the time of RRSO is essential.

Alternatively to RRSO, a strategy consisting of risk-reducing salpingectomy with delayed oophorectomy had been put forward in several publications. Before introducing this strategy, we asked 39 Dutch BRCA1/2 mutation carriers and 23 healthcare professionals in the field of hereditary cancer how to move on with the growing body of evidence for the fallopian tube as site of origin of ovarian cancer and its role in risk-reducing surgery. In this qualitative study reported in chapter $\mathbf{5}$, we identified barriers and facilitators for salpingectomy with delayed oophorectomy and for participation in a clinical trial on this topic during focus groups and in-depth semi-structured interviews. Among BRCA1/2 mutation carriers, main barriers for risk-reducing salpingectomy with delayed oophorectomy were seriousness of ovarian cancer, family history, and previous breast cancer. Among professionals, delay of the risk-reducing effect of oophorectomy on breast cancer risk and a second operation were recognized as main barriers. Both BRCA1/2 mutation carriers and professionals found uncertainty about the effect of the alternative strategy and the fact that women often easily decide to undergo RRSO important barriers. The main facilitator mentioned by both was postponement of surgical menopause. For trial participation, BRCA1/2 mutation carriers would experience a randomized study design as a insurmountable barrier. Professionals identified two facilitators: uniform counseling and willingness of BRCA1/2 mutation carriers to participate in trials. Most BRCA1/2 mutation carriers and professionals were willing to consider participation in a future non-randomized study.

In attempt to answer the question on ovarian carcinoma risks under the alternative risk-reducing strategy, we modeled the cumulative ovarian carcinoma risks after risk-reducing salpingectomy at various ages with delayed oophorectomy several years later and compared these estimates to those after RRSO in chapter $\mathbf{6}$. We extracted the input parameters for this Cox proportional hazard model from the (indirect) evidence available. The estimated cumulative ovarian cancer risks at age 70 years for risk-reducing salpingectomy with delayed oophorectomy are highest for 
BRCA1 mutation carriers undergoing surgeries at higher age. Maximum increase in point estimates (from $1.8 \%$ to $4.1 \%$ ) occurs in a 40-year-old BRCA1 mutation carriers undergoing oophorectomy at age 45 years after non-protective salpingectomy instead of RRSO at age 40 years. In the bestcase scenario, assuming $65 \%$ risk reduction by salpingectomy and $96 \%$ by RRSO, point estimates increase (from $1.8 \%$ to $2.6 \%$ ) or decrease (from 3.4\% to 3.3\%) depending on age. In the worst-case scenario for BRCA2, point estimates maximally increase from $0.6 \%$ to $1.8 \%$ in 45 -year-old carriers when oophorectomy is performed at age 50 years instead of RRSO at age 45 years. In the best-case scenario, point estimates increase (from 1.3\% to 1.5\%) or decrease (from 1.5 to 1.3\%). We concluded that differences in estimated ovarian cancer risks between RRSO and salpingectomy with delayed oophorectomy are small, even if salpingectomy is ineffective.

The findings in aforementioned chapters resulted in the design of a clinical preference trial, the TUBA study. The outline of the study protocol is described in chapter $\mathbf{7}$ and participants have been recruited since January 2015. It concerns a multicenter non-randomized trial conducted in 11 Dutch centers for hereditary cancer. Eligible patients are premenopausal BRCA1/2 mutation carriers who completed childbearing without (a history of) ovarian carcinoma. Participants choose between standard RRSO at age 35-40 (BRCA1) or 40-45 (BRCA2) and the alternative strategy (salpingectomy upon completion of childbearing and delayed oophorectomy at age 40-45 (BRCA1) or 45-50 (BRCA2)). Women who opt for early salpingectomy but do not want to postpone oophorectomy beyond the currently recommended age are included as well. Primary outcome measure is menopause-related quality of life. Secondary outcome measures are ovarian and breast carcinoma incidence, surgery-related morbidity, histopathology, cardiovascular risk factors and diseases, and cost-effectiveness. Data will be analyzed using mixed model data analysis.

Making the choice between standard RRSO and salpingectomy with delayed oophorectomy can be complex. Therefore, we developed a paper patient decision aid that combines evidence-based information with patient preferences to facilitate this complex decision-making process (chapter $\mathbf{8}$ ). The patient decision aid was systematically developed in an iterative process of prototype development, pilot testing by both patients and clinicians, and revisions. Information included in the patient decision aid was based on available literature and current guidelines. The development process was in accordance with the International Patient Decision Aid Standards (IPDAS) quality criteria and supervised by a multidisciplinary steering group. Finally, two paper patient decision aids were developed: one for BRCA1, one for BRCA2. They both contained a general introduction, three chapters, and a step-by-step plan containing a personal value clarification worksheet and underwent four rounds of pilot testing and revisions. Risk communication and information about premature menopause and hormone replacement therapy were the most revised items. The patient decision aid fulfills 37 of 43 IPDAS criteria for content and development process. Effectiveness has not been evaluated yet. Both BRCA1/2 mutation carriers and professionals are willing to use or offer the patient decision aid for the choice between RRSO and salpingectomy with delayed oophorectomy. It has been found clear, balanced and comprehensible. Future testing among patients facing the decision should point out its effectiveness in improving decision-making. 
At last, the content of this thesis is discussed in broader sense in chapter $\mathbf{9}$. The main focus of this chapter is on the difficulty of estimating, interpreting, and personalizing cancer risks among BRCA1/2 mutation carriers. Besides, possibilities for early detection of ovarian carcinoma are passed in revue as well as other implications and suggestions for the future. 


\section{SAMEMVATTIVG}

Dit proefschrift gaat over een alternatieve methode om het risico op eierstokkanker van BRCA1/2 mutatiedraagsters te verlagen. Zoals in hoofdstuk 1 beschreven, hebben deze vrouwen een verhoogd risico op eierstokkanker. Zij krijgen momenteel het advies om een eileider- en eierstokverwijdering (risicoreducerende salpingo-oöphorectomie (RRSO)) te ondergaan rond de leeftijd van 40 jaar. Een RRSO resulteert echter in het plotselinge begin van de (chirurgische) menopauze, hetgeen gevolgen heeft voor de gezondheid op korte en lange termijn. Om de kwaliteit van leven te verbeteren en de effecten van de vervroegde menopauze te verminderen, hebben we de mogelijkheid verkend van een eileiderverwijdering met uitgestelde eierstokverwijdering als alternatief voor RRSO. Hierbij wordt de eierstokfunctie langer behouden en daarmee wordt de overgang uitgesteld.

In hoofdstuk 2 hebben we de literatuur bestudeerd over de kwaliteit van leven van BRCA1/2 mutatiedraagsters die (nog) geen kanker hebben gehad. We hebben gekeken hoe de kwaliteit van leven beïnvloed wordt door verschillende medische keuzes. In algemene zin vonden we dat de kwaliteit van leven niet verschilde van die van vrouwen uit de algemene populatie. Bovendien vonden we geen permanente verandering in kwaliteit van leven door hun keuze voor screening of risicoverlagende operaties. De vervroegde menopauze, veroorzaakt door RRSO, bleek echter de (menopauzegerelateerde) kwaliteit van leven het meest te beïnvloeden. Hormoontherapie kan de overgangsklachten niet altijd volledig wegnemen. Op basis daarvan concludeerden wij dat er een sterke behoefte is aan alternatieven voor RRSO om het risico op eierstokkanker te verlagen enerzijds en aan verbeteringen in begeleiding van patiënten na RRSO anderzijds, bijvoorbeeld op het gebied van hormoontherapie.

We hebben onderzocht hoeveel BRCA1/2 mutatiedraagsters uit het Radboudumc hebben gekozen voor RRSO in hoofdstuk 3, waarbij we ook hebben gekeken naar het moment waarop deze vrouwen de operatie ondergingen. Hiermee wilden we een beeld krijgen van het aantal vrouwen dat mogelijk te maken krijgt met de langetermijneffecten van de vervroegde menopauze. De huidige adviesleeftijd voor RRSO is tussen 35 en 40 jaar voor BRCA1 mutatiedraagsters en tussen 40 en 45 jaar voor BRCA2 mutatiedraagsters. We hebben een retrospectief dossieronderzoek uitgevoerd in het Radboudumc naar BRCA1/2 mutatiedraagsters die gediagnosticeerd en gecounseld waren op onze multidisciplinaire polikliniek Familiaire Tumoren tussen 1999 en 2014. De gegevens van 580 $B R C A 1 / 2$ mutatiedraagsters konden worden geanalyseerd. We vonden dat $98,5 \%$ van de BRCA1 mutatiedraagsters en $97,5 \%$ van de BRCA2 mutatiedraagsters, die ouder waren dan respectievelijk 40 en 45, een RRSO hadden ondergaan. Het overgrote deel van de vrouwen, die hun mutatie wisten op de bovengenoemde adviesleeftijd, onderging de RRSO ook op de adviesleeftijd: 90,8\% van de BRCA1 mutatiedraagsters vóór 41-jarige leeftijd en 97,3\% van de BRCA2 mutatiedraagsters vóór 46-jarige leeftijd. Het merendeel van de BRCA1/2 mutatiedraagsters ondergaat de RRSO dus vóór de natuurlijke menopauze. Dit benadrukte nogmaals hoe belangrijk het is om manieren 
te zoeken om de hormonale gevolgen van de vervroegde menopauze te minimaliseren door enerzijds hormoontherapie te optimaliseren en anderzijds een alternatief te vinden voor RRSO. Ondanks RRSO krijgen sommige BRCA1/2 mutatiedraagsters alsnog buikvlieskanker. Buikvlieskanker valt ook onder de verzamelnaam 'eierstokkanker'en kan ook na een RRSO nog optreden aangezien het buikvlies bij een preventieve operatie niet kan worden verwijderd. In hoofdstuk 4 hebben we casus verzameld van BRCA1/2 mutatiedraagsters die inderdaad buikvlieskanker kregen, nadat zij al een risicoverlagende operatie hadden ondergaan (meestal RRSO). Voor dit hoofdstuk hebben we in de literatuur gezocht naar publicaties waarin één of meer van deze casus werden beschreven en we hebben desbetreffende auteurs gevraagd om aanvullende gegevens. Deze casus hebben we vergeleken met een controlegroep van BRCA1/2 mutatiedraagsters die tussen 2014 en 2016 een RRSO ondergingen in het Radboudumc. In totaal hebben we 36 casus vanuit de literatuur verzameld waarbij het in 31 gevallen ging om BRCA1 mutatiedraagsters (86,1\%). De mediane leeftijd tijdens de risicoverlagende operatie was 52 jaar (spreiding 30-71 jaar) en de mediane leeftijd waarop buikvlieskanker werd vastgesteld was 60 jaar (spreiding 37-75 jaar). De mediane tijd die verstreek tussen de risicoverlagende operatie en de diagnose buikvlieskanker was 54,5 maand (spreiding 11292 maanden). Buikvlieskanker was meestal van het hooggradig sereuze type. Voor 8 casus waren de details over de volledig onderzochte eileiders beschikbaar: 5 hiervan (62,5\%) toonden een sereus tubair intra-epitheliaal carcinoom (STIC), dat beschouwd wordt als een voorstadium van eierstokkanker. De eileiders van één casus hadden atypisch epitheel. De BRCA1/2 mutatiedraagsters die na een risicoverlagende operatie nog buikvlieskanker ontwikkelden waren significant ouder ten tijde van de ingreep en hadden meer STICs dan de vrouwen uit de controlegroep. We concludeerden dat buikvlieskanker na een risicoverlagende operatie vooral voorkomt bij BRCA1 mutatiedraagsters, binnen 5 jaar. De gegevens suggereren dat RRSO op de adviesleeftijd leidt tot een lagere kans op buikvlieskanker nadien dan een RRSO op oudere leeftijd. De bevindingen van dit onderzoek kunnen ons helpen bij het voorlichten van BRCA1/2 mutatiedraagsters. Verder wordt het belang onderstreept van een volledige eileiderverwijdering tijdens de eierstokverwijdering en van het nauwkeurig microscopisch onderzoek van de verwijderde eileiders en eierstokken.

In verschillende publicaties wordt een alternatief voor RRSO gesuggereerd, bestaande uit een eileiderverwijdering met uitgestelde eierstokverwijdering. Over de beste manier om die alternatieve methode te onderzoeken bestond echter geen duidelijkheid. Wij hebben 39 BRCA1/2 mutatiedraagsters en 23 zorgprofessionals van de poliklinieken Familiaire tumoren in Nederland om advies gevraagd hoe verder te gaan met het steeds duidelijkere bewijs dat eierstokkanker in de eileider ontstaat. Een belangrijke vraag was wat de gevolgen hiervan zouden kunnen zijn voor risicoverlagende operaties. In deze kwalitatieve studie, die wordt beschreven in hoofdstuk 5, hebben we barrières en faciliterende factoren voor de alternatieve methode en voor deelname aan een klinisch onderzoek geïnventariseerd. Dit werd gedaan tijdens focusgroepen en individuele semigestructureerdeinterviews.Voor de BRCA1/2 mutatiedraagsters waren de belangrijkste barrières voor eileiderverwijdering met uitgestelde eierstokverwijdering: de ernst van eierstokkanker, een 
belaste familieanamnese en het gehad hebben van borstkanker. Voor professionals waren het uitstellen van het (vermeende) gunstige effect van RRSO op borstkanker en het ondergaan van twee operaties de belangrijkste barrières. Zowel BRCA1/2 mutatiedraagsters als de professionals vonden de onzekerheid over het risicoverlagende effect van de eileiderverwijdering en het feit dat vrouwen over het algemeen relatief makkelijk besluiten tot het ondergaan van een RRSO belangrijke barrières. De belangrijkste faciliterende factor die door beide groepen werd benoemd was de mogelijkheid tot het uitstellen van de menopauze. Ten aanzien van deelname aan een klinisch onderzoek naar de alternatieve methode, gaven BRCA1/2 mutatiedraagsters aan dat een gerandomiseerde onderzoeksopzet, waarin het type behandeling wordt bepaald door loting, een reden zou zijn om zeker niet deel te nemen. Volgens de zorgprofessionals waren er twee factoren die faciliterend zouden kunnen werken: landelijk een uniforme manier van voorlichten en de bereidheid van BRCA1/2 mutatiedraagsters om deel te nemen aan wetenschappelijke onderzoeken. De meeste BRCA1/2 mutatiedraagsters en zorgprofessionals waren bereid om deelname aan zo'n niet-gerandomiseerd onderzoek te overwegen.

Om het risico op eierstokkanker zo goed mogelijk in te schatten, hebben we een model gemaakt voor het risico na de eileiderverwijdering en latere eierstokverwijdering op verschillende leeftijden en dat vergeleken met het risico na RRSO. Deze schattingen worden beschreven in hoofdstuk 6 . Het Cox proportional hazard model hebben we gebaseerd op gegevens uit de literatuur. Het geschatte eierstokkankerrisico tot de leeftijd van 70 jaar voor de alternatieve methode was het hoogst voor BRCA1 mutatiedraagsters die beide operaties (eileiderverwijdering en latere eierstokverwijdering) op hogere leeftijd ondergaan. De grootste toename van het geschatte risico (puntschatting van 1,8\% naar 4,1\%) werd gevonden voor een 40-jarige BRCA1 mutatiedraagster die zou kiezen voor eierstokverwijdering op 45-jarige leeftijd na een eerdere eileiderverwijdering die geen beschermend effect had, in plaats van een RRSO op 40-jarige leeftijd. In het beste geval, uitgaande van 65\% risicoverlaging door de eileiderverwijdering en 96\% risicoverlaging door de RRSO, zouden de puntschattingen van het cumulatief risico iets stijgen (van 1,8\% naar 2,6\%) of dalen (van 3,4\% naar 3,3\%), afhankelijk van de leeftijd. Voor een 45-jarige BRCA2 mutatiedraagster stijgt de puntschatting van het cumulatief risico in het slechtste geval van 0,6\% naar 1,8\% indien zij op 50-jarige leeftijd de uitgestelde eierstokverwijdering ondergaat na een ineffectieve eileiderverwijdering in plaats van een RRSO op 45-jarige leeftijd. In het beste geval stijgen (van 1,3 naar 1,5\%) of dalen (van 1,5\% naar 1,3\%) de puntschattingen gering, afhankelijk van de leeftijd. We concludeerden dat de verschillen in het geschatte risico op eierstokkanker tussen RRSO en eileiderverwijdering met uitgestelde eierstokverwijdering klein zijn, zelfs als de eileiderverwijdering het risico op eierstokkanker niet verlaagt.

De bevindingen uit de voorgaande hoofdstukken hebben geresulteerd in de opzet van een klinisch onderzoek, de TUBA studie: "Early salpingectomy (TUbectomy) with delayed oophorectomy to improve quality of life as alternative for risk-reducing salpingo-oophorectomy in $\boldsymbol{B} R C \boldsymbol{A} 1 / 2$ mutation carriers". De samenvatting van het onderzoeksprotocol vormt hoofdstuk 7 . Sinds januari 
2015 kunnen BRCA1/2 mutatiedraagsters meedoen aan dit multicenter, niet-gerandomiseerde onderzoek in 11 Nederlandse ziekenhuizen met een polikliniek Familiaire tumoren. BRCA1/2 mutatiedraagsters kunnen meedoen aan de TUBA studie als zij premenopauzaal zijn, hun kinderwens voltooid hebben en geen eierstokkanker hebben (gehad). Deelneemsters maken zelf de keuze voor hetzij de standaard RRSO op de adviesleeftijd (35-40 jaar voor BRCA1 mutatiedraagsters en 40-45 jaar voor BRCA2 mutatiedraagsters), hetzij de alternatieve methode (eileiderverwijdering na het voltooien van de kinderwens met uitgestelde eierstokverwijdering tussen 40-45 jaar (BRCA1) of tussen 45-50 jaar (BRCA2)). Vrouwen die kiezen voor een eileiderverwijdering, maar de eierstokverwijdering niet willen uitstellen tot voorbij de adviesleeftijd, kunnen ook deelnemen aan het onderzoek. Menopauzegerelateerde kwaliteit van leven is de primaire uitkomstmaat. De incidentie van borst- en eierstokkanker, complicaties rondom de operaties, histopathologisch onderzoek van de verwijderde weefsels, risicofactoren op en incidentie van hart- en vaatziekten, en kosteneffectiviteit zijn secundaire uitkomstmaten.

Het maken van de keuze tussen de standaard RRSO en eileiderverwijdering met uitgestelde eierstokverwijdering kan zeer complex zijn. Om dit keuzeproces te ondersteunen hebben we een papieren keuzehulp ontwikkeld waarin wetenschappelijk bewijs gecombineerd wordt met de voorkeuren van de patiënt (hoofdstuk 8). De keuzehulp is op systematische wijze ontwikkeld door middel van een herhaaldelijk proces van ontwikkelen, testen door patiënten en artsen, en reviseren. De informatie in de keuzehulp is gebaseerd op de literatuur en bestaande richtlijnen. Bij de ontwikkeling zijn de IPDAS criteria (International Patient Decision Aid Standards) leidend geweest en dit ontwikkelingsproces werd gesuperviseerd door een multidisciplinaire stuurgroep. Uiteindelijk zijn er twee keuzehulpen tot stand gekomen: één voor BRCA1 en één voor BRCA2. Beide bestaan uit een algemene inleiding, drie hoofdstukken en een stappenplan met een werkblad om de persoonlijke voorkeuren van de gebruiker te verhelderen. In totaal zijn er vier rondes van testen en reviseren doorlopen. Risicocommunicatie en de informatie over de vroegtijdige overgang en hormoontherapie waren de onderwerpen die het meest gereviseerd werden. De uiteindelijke keuzehulpen voldoen aan 37 van de 43 IPDAS criteria voor de inhoud en het ontwikkelingsproces. De effectiviteit is nog niet onderzocht. Zowel BRCA1/2 mutatiedraagsters als de artsen die meededen in de testrondes waren bereid om de keuzehulpen te gaan gebruiken. Ze werden als duidelijk, gebalanceerd en begrijpelijk bestempeld. De volgende stap zal zijn om de keuzehulpen aan te bieden aan BRCA1/2 mutatiedraagsters, die inderdaad voor de keuze staan tussen RRSO en eileiderverwijdering met uitgestelde eierstokverwijdering, en te evalueren in hoeverre het gebruik van deze keuzehulpen inderdaad de besluitvorming verbetert.

Tot slot wordt in hoofdstuk 9 de inhoud van dit proefschrift in bredere zin bediscussieerd. Dit hoofdstuk richt zich vooral op de complexiteit van het schatten, interpreteren en individualiseren van de kankerrisico's van BRCA1/2 mutatiedraagsters. Verder worden mogelijkheden van vroege opsporing van eierstokkanker kort besproken, evenals andere suggesties voor toekomstig onderzoek. 


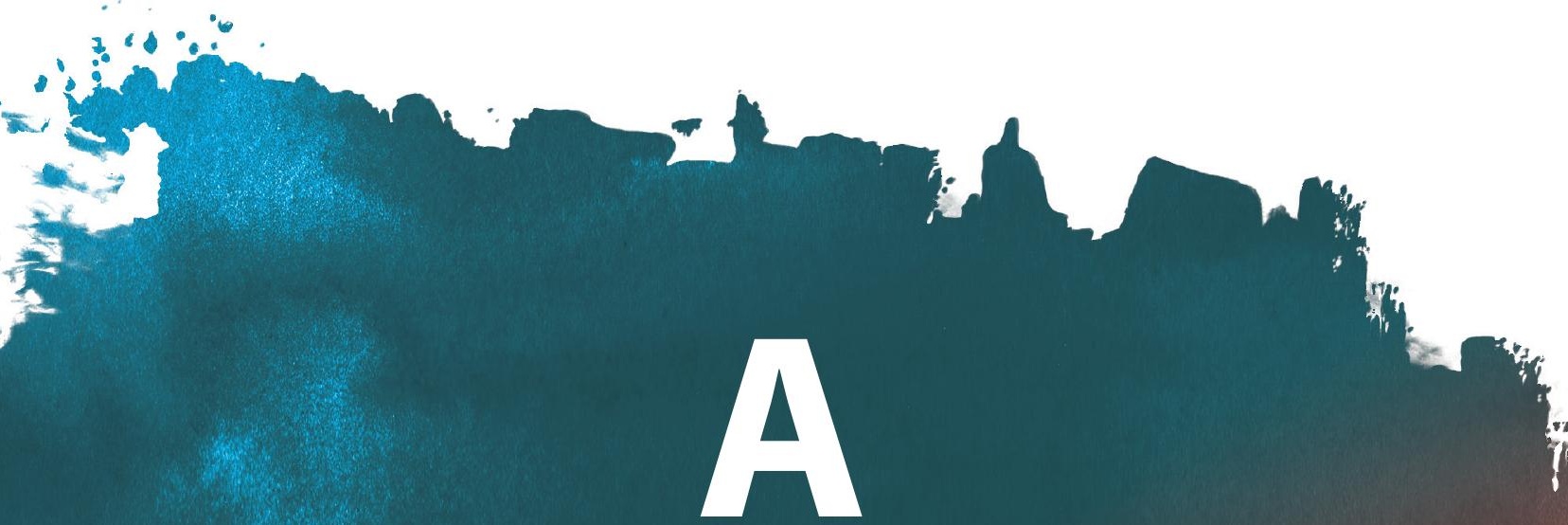

Bibliography

Dankwoord

Curriculum Vitare

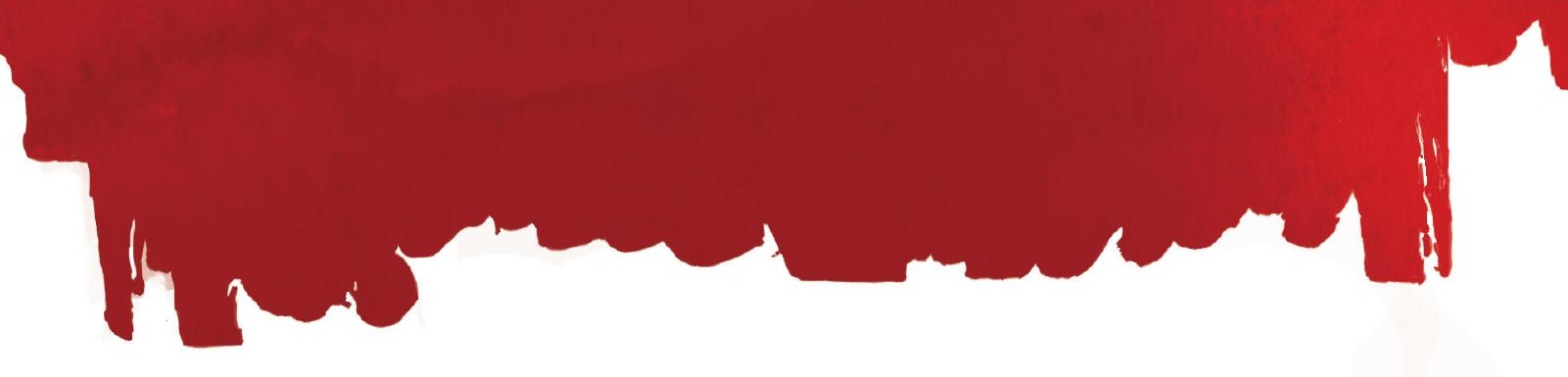




\section{BIBLIOGRAPAY}

Samimi G, Bernardini MQ, Brody LC, Caga-Anan CF, Campbell IG, Chenevix-Trench G, Couch FJ, Dean M, de Hullu JA, Domchek SM, Drapkin R, Spencer Feigelson H, Friedlander M, Gaudet MM, Harmsen MG, Hurley K, James PA, Kwon JS, Lacbawan F, Lheureux S, Mai PL, Mechanic LE, Minasian L, Myers ER, Robson ME, Ramus SJ, Rezende LF, Shaw PA, Slavin TP, Swisher EM, Takenaka M, Bowtell DD, Sherman ME. Traceback: A proposed framework to increase identification and genetic counseling of BRCA1 and BRCA2 mutation carriers through family-based outreach. J Clin Oncol. 2017 Apr 11 [Epub ahead of print]

Harmsen MG, Arts-de Jong M, Horstik K, Manders P, Massuger LF, Hermens RP, Hoogerbrugge $\mathrm{N}$, Woldringh $\mathrm{GH}$, de Hullu JA. Very high uptake of risk-reducing salpingo-oophorectomy in BRCA1/2 mutation carriers: A single-center experience. Gynecol Oncol. 2016 Oct;143(1):113-9.

Harmsen MG, IntHout J, Arts-de Jong M, Hoogerbrugge N, Massuger LF, Hermens RP, de Hullu JA. Salpingectomy With Delayed Oophorectomy in BRCA1/2 Mutation Carriers: Estimating Ovarian Cancer Risk. Obstet Gynecol. 2016 Jun;127(6):1054-63.

Harmsen MG, Hermens RP, Prins JB, Hoogerbrugge N, de Hullu JA. How medical choices influence quality of life of women carrying a BRCA mutation. Crit Rev Oncol Hematol. 2015 Dec;96(3):555-68.

Harmsen MG, Arts-de Jong M, Hoogerbrugge N, Maas AH, Prins JB, Bulten J, Teerenstra S, Adang EM, Piek JM, van Doorn HC, van Beurden M, Mourits MJ, Zweemer RP, Gaarenstroom KN, Slangen $B F$, Vos MC, van Lonkhuijzen LR, Massuger LF, Hermens RP, de Hullu JA. Early salpingectomy (TUbectomy) with delayed oophorectomy to improve quality of life as alternative for risk-reducing salpingo-oophorectomy in BRCA1/2 mutation carriers (TUBA study): a prospective non-randomised multicentre study. BMC Cancer. 2015 Aug 19;15(1):593.

Arts-de Jong M, Harmsen MG, Hoogerbrugge N, Massuger LF, Hermens RP, de Hullu JA. Riskreducing salpingectomy with delayed oophorectomy in BRCA1/2 mutation carriers: patients' and professionals' perspectives. Gynecol Oncol. 2015 Feb;136(2):305-10.

Kaandorp JJ, Derks JB, Oudijk MA, Torrance HL, Harmsen MG, Nikkels PGJ, van Bel F, Visser GHA, Giussani DA. Antenatal Allopurinol reduces hippocampal brain damage after acute birth asphyxia in late gestation fetal sheep. Reprod Sci. 2014 Feb;21(2):251-9. 


\section{Submitted papers}

Harmsen MG, Piek JMJ, Bulten J, Casey MJ, Rebbeck TR, Mourits MJ, Greene MH, Slangen BFM, van Beurden M, Massuger LFAG, Hoogerbrugge N, de Hullu JA. Peritoneal carcinomatosis after risk-reducing surgery in BRCA1/2 mutation carriers: new implications for gynecologic counseling.

Harmsen MG, Hoogerbrugge N, van Doorn HC, Gaarenstroom KN, Vos MC, Massuger LFAG, de Hullu JA, Hermens RPMG. A patient decision aid for risk-reducing surgery in premenopausal $B R C A 1 / 2$ mutation carriers: development process and pilot testing. 


\section{CURRICULLM VITAE}

Marline Harmsen werd geboren op 19 februari 1987 als dochter van Evert Harmsen en Marja Stockmann en zusje van Joep. Zij is geboren en getogen in Nieuwegein, waar zij in 2004 op het Oosterlicht College haar gymnasiumdiploma cum laude behaalde. In datzelfde jaar begon zij met de studie geneeskunde aan de Universiteit van Utrecht. In 2008 liep zij een keuzecoschap Tropengeneeskunde in het Namanyere Designated District Hospital in Namanyere, Rukwa, Tanzania. Haar liefde voor de verloskunde en gynaecologie werd aangewakkerd door haar reguliere coschap in het Antonius ziekenhuis te Nieuwegein, haar semi-artsstage in het Amphia ziekenhuis te Breda en haar wetenschappelijke stage op de afdeling Verloskunde van het Wilhelmina Kinderziekenhuis in Utrecht. Na haar afstuderen in 2010 en een reis door Latijns Amerika ging zij op zoek naar een baan als ANIOS Verloskunde en Gynaecologie en werkte in de tussentijd als research medewerker op de afdeling Research \& Development Cardiologie van het Antonius ziekenhuis in Nieuwegein. In januari 2012 begon zij als ANIOS Verloskunde \& Gynaecologie in het Rijnstate ziekenhuis in Arnhem, waar zij overtuigd raakte van haar keuze voor dit specialisme. Marline begon naast haar klinische baan met het uitvoeren van wetenschappelijk onderzoek onder begeleiding van Dr. Joanne de Hullu. Dit resulteerde in juni 2014 in een voltijd aanstelling als promovenda op de afdeling Verloskunde \& Gynaecologie van het Radboudumc in Nijmegen. Het resultaat van dit promotietraject heeft u nu in handen. Dit najaar zal zij starten met de opleiding tot gynaecoloog in het Rijnstate ziekenhuis in Arnhem (opleiders Dr. F.P.H.L.J. Dijkhuizen en Dr. A.C. Bolte).

Al vanaf haar achtste staat Marline in haar vrije tijd op het honk- of softbalveld, waar zij haar favoriete spelletje tot op hoog niveau heeft beoefend. Verder grijpt zij iedere gelegenheid aan om met een rugzak naar verre en tropische bestemmingen af te reizen. Marline woont in Nijmegen sinds eind 2013. 


\section{DAHKWOORD}

Mijn proefschrift is af! Uiteraard heb ik dit niet alleen gedaan en ik wil een ieder die een bijdrage heeft geleverd heel hartelijk danken.

Allereerst wil ik graag de BRCA1/2 mutatiedraagsters bedanken die geheel belangeloos bereid waren om vanuit huis of in het Radboudumc mee te denken over de rol van de tubectomie, de opzet van de TUBA studie, de patiënteninformatie en/of de keuzehulp. Ook de input van de patient advocates van Borstkankervereniging Nederland was zeer waardevol, waarvoor dank. Alle vrouwen die meedoen aan de TUBA studie: hartelijk dank voor jullie interesse en deelname!

Zonder goede begeleiding komt er geen proefschrift: wat heb ik het getroffen met mijn vierkoppige promotieteam! Prof.dr. L.F.A.G. Massuger, beste Leon, ik denk dat er weinig mensen zijn, die een vergelijkbaar drukke baan hebben en het toch presteren om de hele dag fluitend en neuriënd rond te lopen! Overigens weet je daardoor wel altijd wanneer je in de buurt bent en dus wanneer er even snel een handtekening gescoord kan worden of wanneer je kunt proberen illegaal in te breken in je overvolle agenda. Ik heb bewondering voor je kennis, rust, daadkracht en creativiteit. Met je kritische blik wist je de vinger vaak op de zere plek te leggen en projecten tijdig bij te sturen. Ik wil je enorm bedanken voor je vertrouwen.

Prof.dr. N. Hoogerbrugge, beste Nicoline, ik bewonder je bevlogenheid waarmee je probeert zo veel mogelijk mensen met erfelijke aanleg voor kanker op te sporen en de zorg voor deze populatie te verbeteren. Als een artikel focus miste, wist jij mij met enkele opmerkingen de boodschap weer helder te laten krijgen door terug te gaan naar de basis. Dit leidde altijd tot een veel betere volgende versie, waarvoor ik je wil bedanken. Daarnaast hebben we ook meermaals hard gelachen. De treinrit naar Schiphol op weg naar het BRCA symposium in Montreal is me daarvan het meest bijgebleven: een Katja Apenkop zal nooit meer hetzelfde zijn!

Dr. J.A. de Hullu, beste Joanne, een ding is duidelijk: zonder jou was dit proefschrift er nooit geweest. Wat ben jij een voorbeeld voor mij! Alles lijkt bij jou op de eerste plaats te komen: familie, patiëntenzorg, onderzoek, sport, maar zeker ook het wel en wee van je promovendi. Hoe je dit alles weet te combineren is mij een raadsel, maar ik kan er alleen maar oneindig veel respect voor hebben en hopen dat ik dat ook kan "als ik later groot ben", al is het maar half zo goed. Verder kunnen niet veel promovendi zeggen dat ze met "de baas" vijf dagen in een Airbnb hebben doorgebracht met maar één huissleutel, maar wat was het ontspannen (op het half uurtje loopband op de eerste dag na...). Bedankt dat je me deze kans hebt geboden en nog veel meer dank voor het grenzeloze vertrouwen en de persoonlijke interesse die je vanaf de eerste dag in mij hebt gehad, je bent goud waard! 
Dr. R.P.M.G. Hermens, beste Rosella, jouw expertise vanuit een hele andere hoek was een zeer welkome aanvulling op het team. Pogingen om ergens niet over uit te weiden of "overheen te schrijven" sneuvelden steevast als jij het stuk onder ogen kreeg en daarnaast bracht jij altijd de structuur terug in een artikel. Onze afspraken (als ze in je agenda stonden tenminste ;-)) waren constructief en efficiënt, waardoor er ook genoeg tijd overbleef voor een lolletje. Door de vakantiefoto's en kleine of grotere overwinningen die gedeeld werden in de TUBA groepsapp bleven we goed van elkaar op de hoogte. Hartstikke bedankt voor alles!

Beste Marieke, doordat jij een tweede persoon zocht voor het coderen en analyseren van de interviews en focusgroepen uit hoofdstuk 5, ben ik in dit onderzoek gerold. Mede door al jouw voorwerk, heb ik een zeer voorspoedig promotietraject gehad. Hiervoor ben ik je eeuwig dankbaar!

Lieve Christel, wat een ongekende luxe om als arts-onderzoeker samen te mogen werken met een research verpleegkundige, want wat heb jij mij ongelooflijk veel werk uit handen genomen! Zonder al jouw werk voor de TUBA studie, zou nog niet de helft van dit proefschrift nu klaar zijn. Daarnaast was het gewoon ook altijd leuk om bij te kletsen in de auto, samen te zeuren over alle regelgeving, oplossingen te bedenken voor allerlei grote en kleine problemen en het land door te crossen naar al onze tijdelijke werkgevers. Dankjewel!

Uiteraard wil ik alle medeauteurs bedanken die hun bijdrage hebben geleverd aan een of meerdere hoofdstukken van dit proefschrift. Dr. J.M.J. Piek, beste Jurgen, het is een eer om zo nauw samen te werken met degene die ruim 15 jaar geleden de tuba voor het eerst aanwees als mogelijke boosdoener in het ontstaan van eierstokkanker bij BRCA1/2 mutatiedraagsters. Ik ben benieuwd of ik hier net zo'n "tubafetisj" aan overhoud als jij! Dr. J. in 't Hout, beste Joanna, zonder jouw statistische expertise was hoofdstuk 6 er niet geweest, dankjewel!

Ook al zullen de resultaten van de TUBA studie nog even op zich laten wachten, ik wil toch graag alle lokale onderzoekers van de deelnemende centra bedanken voor alle tijd die jullie er tot nu toe al in hebben gestopt. Hierbij gaat mijn dank natuurlijk minstens zo veel uit naar alle betrokken research verpleegkundigen en verpleegkundig specialisten die veel werk verzetten voor de TUBA studie, evenals naar de pathologen die de preparaten nauwkeurig bekijken en naar de klinisch genetici die het bestaan van de studie noemen tijdens hun counseling. Ik kan niet wachten tot de resultaten er zijn!

Beste gynaecologisch oncologen van het Radboudumc, heel hartelijk dank voor het counselen en includeren van patiënten voor de TUBA studie, alsmede voor het uitvoeren van de extra handelingen die van jullie worden gevraagd tijdens de laparoscopieën. Beste Bertho, dankjewel voor je inzet voor het project waarmee ik bijkluste bij de benigne gyn, hopelijk komen daar ook mooie resultaten uit (en natuurlijk minstens zoveel dank voor de recordpogingen op de skipiste). 
Lieve dames van het secretariaat: dank voor jullie ondersteuning. Yvonne, Els, Wilma en Ans, jullie maken het leven van een onderzoeker net een beetje makkelijker!

Beste gynaecologen, assistenten, verloskundigen en verpleegkundigen uit het Rijnstate ziekenhuis, mede door jullie is mijn enthousiasme voor het vak na mijn studie verder aangewakkerd. Ik heb dan ook heel veel zin om dit najaar weer terug te komen en hopelijk beland ik dan weer in datzelfde warme bad (met Zwitsal) als waar ik in juni 2014 letterlijk uitklom.

Lieve "Rijnstatematties", wat heb ik genoten van onze 3-gangendiners de afgelopen jaren! Door alle verhalen (en veel oude koeien) had ik tijdens die etentjes toch nog het gevoel een beetje in de kliniek te zitten.

Lieve (ex-)tuinbewoners, niets is zo belangrijk als leuke collega's! Jullie waren de belangrijkste reden dat ik niet deed aan "thuiswerken" en zonder jullie had mijn onderzoekstijd er heel anders uitgezien. Bedankt voor alle theemomentjes, lunches, vrijmibo's, weekendjes, (water)skitripjes, pubquizzen, sushidiners, (vierdaagse)feestjes, (extended) congressen etcetera. Jullie zijn top! Lieve Michelle, de Radboudumc server zal het moeilijk gehad hebben met de hoeveelheid e-mails die wij elkaar per dag stuurden terwijl we zo'n acht meter van elkaar af zaten. Daarbuiten ging de communicatie meestal in hetzelfde tempo door via WhatsApp. Kortom, wat hebben wij de afgelopen 2,5 jaar veel gedeeld en dan laat ik alle gedeelde bedden nog buiten beschouwing: Nederlandse, Oostenrijkse, Franse, Portugese, Colombiaanse... Gelukkig gaan we elkaar nog veel zien!

Lieve Gang, ongelooflijk dat we alweer 13 jaar geleden van school gingen. Gaaf dat er inmiddels een volledige minigang is en dat we elkaar af en toe nog zien. We zijn een bijzonder clubje! Lieve Gies, ik ken weinig mensen die zo kunnen stralen als jij! Knap hoe je weet te kiezen vanuit jezelf en voor dingen waar je blij van wordt. Ik koester onze memorabele stapavondjes die meestal eindigen in een zeer dubieuze kroeg, maar ook onze saunatraditie met Roos. Laten we er nog vele jaren aan vastplakken, je bent een schat! Lieve Roos, wat verschillen wij van elkaar! Wellicht is dat de kracht van onze vriendschap die op de basisschool nog wat wisselvallig was, maar vanaf de brugklas alleen maar beter werd. Van jou heb ik geleerd dat niets onmogelijk is en met jou is het nooit saai. Van samen GoGo bellen tot onszelf uitnodigen op de after party van de Boliviaanse equivalent van the Stones: het gebeurt alleen als jij erbij bent. Dankjewel voor wie je bent!

Lieve Ranger ladies, wat zijn jullie toch leuk! Ik ben blij dat jullie mij al twee jaar in jullie studententeam gedogen en dat we een paar keer per week met z'n allen de mooiste sport ter wereld kunnen beoefenen, maar ook dat we veel leuke teamuitjes en Rangerborrels hebben. Lieve Mien en Rens, jammer dat ik te ver weg woon om nog samen met jullie te softballen, maar ik koester onze vriendschap waarvoor we circa drie keer per week gedurende bijna 10 jaar een goede basis hebben gelegd! 
Lieve Bruutjes, dat we als club na bijna 13 jaar af en toe nog compleet zijn, vind ik echt heel bijzonder! Op naar de volgende life events en wanneer mogen we weer op lustrumreis? Lieve Val, wat kom ik graag bij jullie over de vloer en wat ben jij een held! Lieve Lou, ook al is Zwitserland niet naast de deur, als wij elkaar na maanden/jaren weer zien, is het gelijk goed! Lieve Celine, fijn dat we allebei in Nijmegen wonen!

Lieve paranimfen, waar het organiseren van een assistentenweekend allemaal niet toe kan leiden! $\mathrm{Na}$ alle etentjes en borrels met z'n drieën was de keuze voor mijn paranimfen snel gemaakt. Lieve Yalckie, wat keek ik tegen jou op toen ik een maand na jou in het Rijnstate begon, want "jij kon al zo veel"! Sindsdien zijn we ruim vijf jaar naaste collega's geweest, maar wisten we elkaar gelukkig ook buiten het ziekenhuis vaak te vinden (doorgaans bij Samson of Anneke...). Lieve Thijs, wat is het toch fijn dat jij zo eerlijk en direct ben, geen onderwerp onbesproken laat (ook alvorens de Karmeliet Tripels inslaan) en ook zo houdt van een terrasje in de zon. Lieverds, laten we Assistentenweekend Volendam 2014 nog lang blijven evalueren!

Lieve opa en oma Harmsen, ik vind het ongelooflijk bijzonder dat jullie het gereedkomen van mijn proefschrift nog mogen meemaken. Ik heb veel bewondering voor jullie en geniet nog altijd van jullie prachtige verhalen over vroeger.

En tot slot, wie of wat zou ik zijn zonder het fantastische gezin waar ik uit kom? Lieve Joep, super mooi om te zien hoe snel jij carrière aan het maken bent in de Amsterdamse ziekenhuizen! Wij begrijpen elkaars humor als geen ander en ik waardeer je creativiteit, handigheid en gastvrijheid met bijbehorende kookkunsten (als ik weer eens een slaapplaats zoek in Amsterdam bijvoorbeeld). Ik zeg altijd vol trots dat ik een grote broer heb! Lieve Jud, ik zou me geen leuker, slimmer, gezelliger en grappiger schoonzusje kunnen wensen. Het is altijd leuker als jij erbij bent! Liefste pap en mam, jullie zijn de belangrijkste personen in mijn leven en ik zou mij geen betere ouders kunnen bedenken. Jullie onvoorwaardelijke emotionele steun ervaar ik in alles, maar ook de praktische ondersteuning die jullie me nog vaak bieden is goud waard. Ik zou niet weten wat ik zonder jullie moest en mijn dankbaarheid voor alles wat jullie voor me doen en gedaan hebben is eigenlijk niet in woorden uit te drukken. Ik houd van jullie! 